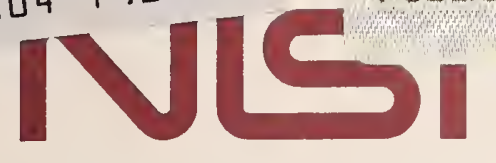

d States Department of Commerce Technology Administration

National Institute of Standards and Technology

NIST Technical Note 1373

\title{
Spherical-Wave Source-Scattering Matrix Analysis of Antennas and Antenna-Antenna Interactions
}

Richard Lindsay Lewis

QC 



\section{Spherical-Wave Source-Scattering Matrix Analysis of Antennas and Antenna-Antenna Interactions}

\section{Richard Lindsay Lewis}

Electromagnetic Fields Division

Electronics and Electrical Engineering Laboratory

National Institute of Standards and Technology

325 Broadway

Boulder, Colorado 80303-3328

July 1995

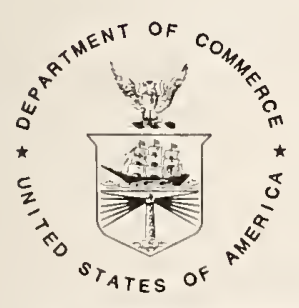

U.S. DEPARTMENT OF COMMERCE, Ronald H. Brown, Secretary TECHNOLOGY ADMINISTRATION, Mary L. Good, Under Secretary for Technology NATIONAL INSTITUTE OF STANDARDS AND TECHNOLOGY, Arati Prabhakar, Director 
National Institute of Standards and Technology Technical Note Natl. Inst. Stand. Technol., Tech. Note 1373, 156 pages (July 1994) CODEN:NTNOEF

\section{U.S. GOVERNMENT PRINTING OFFICE}

WASHINGTON: 1995

For sale by the Superintendent of Documents, U.S. Government Printing Office, Washington, DC 20402-9325 


\section{CONTENTS}

Chapter

I. INTRODUCTION .............................. 1

II. ELECTROMAGNETIC FIELD EXPANSIONS . . . . . . . . . . . . . 5

2.1 Introduction . . . . . . . . . . . . . . . . . . . 5

2.2 Representation of the Fields at the Terminal Surface $S_{0}$ and the Spherical Surface $S \ldots \ldots \ldots \ldots$

2.3 Dyadic Green's Function Representations . . . . . . . . . . . . . . . . . . . . 9

2.4 Orthogonality Relations for the Vector Spherical-Wave Functions . . . . . . . 15

2.5 Conclusions .......................... 18

III. SPHERICAL-WAVE SOURCE-SCATTERING MATRIX REPRESENTATION

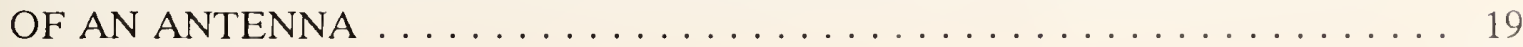

3.1 Introduction . . . . . . . . . . . . . . . . . . . . 19

3.2 Formulation of the Source-Scattering Matrix Representation . . . . . . . . . 19

3.3 Reciprocity Relations . . . . . . . . . . . . . . . . . . . . 20

3.4 Power Gain and Effective Area of an Antenna . . . . . . . . . . . . . . 21

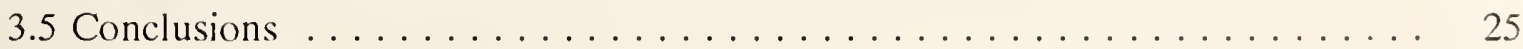

IV. FUNCTIONAL TRANSFORMATIONS INDUCED BY COORDINATE-SYSTEM

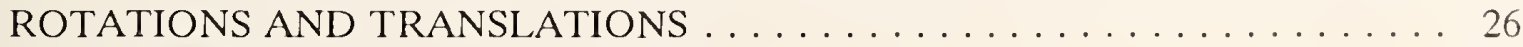

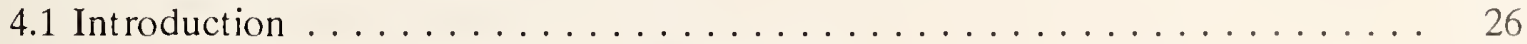

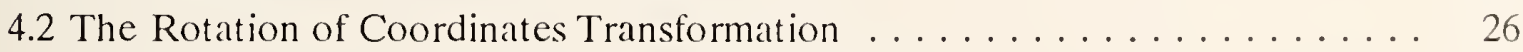

4.3 Application of the Fano-Racah Recursion Formula . . . . . . . . . . . . . 31

4.3.1 The Derivation of a Numerical Calculation Formula . . . . . . . . . 31

4.3.2 Analytical Formula Relating Rotation Coefficients

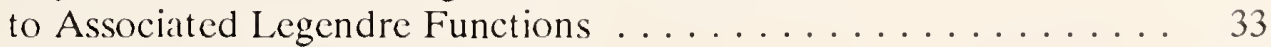

4.4 The Translation of Coordinates Transformation $\ldots \ldots \ldots \ldots \ldots$

4.5 Conclusions . . . . . . . . . . . . . . . . . . . . . . . . . 39

V. SCATTERING MATRIX ANALYSIS OF COUPLED ANTENNAS: GENERAL SOLUTION FOR SYSTEM TWO-PORT . . . . . . . . . . . . . . 40

5.1 Introduction . . . . . . . . . . . . . . . . . . . . 40

5.2 Modal Coefficient-Transformation Solution to the

Coupled Antenna Problem ............................. 45

5.2.1 Heuristic Development of the Two-Port Equations . . . . . . . . . . . 45

5.2.2 Explicit Determination of the Translation Coefficients . . . . . . . . 48

5.2.3 The Two-Port Equation for Oppositely Directed

Transmission ............................. 51

5.2.4 Antenna Coupling Expressed in Terms of Each Antenna's

Spherical-Wave Source-Scattering-Matrix Elements .......... 53

5.3 Conclusions . . . . . . . . . . . . . . . . . . . . . 57 
VI. INTERRELATIONS BETWEEN THE TRANSMISSION EQUATIONS . . . . . 58

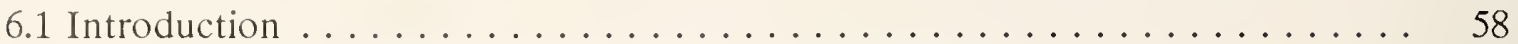

6.2 Establishment of Equalities between the Contragradient

Vector-Wavefunction Translation Coefficients ............. 58

6.3 Equivalence of the Coupling Equations Representing

Oppositely Directed Transmission for the Case of

Reciprocal Antennas .....................

6.4 Application of the Transmission Equations to Near-Field

to Far-Field Antenna Pattern Transformations

(Spherical Scanning) . . . . . . . . . . . . . . . . .

6.4.1 Equivalence of the Transmitting Test-Antenna and

Receiving Test-Antenna Spherical-Scanning

Computer Algorithms . . . . . . . . . . . . . . . . .

6.4.2 Relating Probe Receiving Coefficients in the Doubly-

Primed Coordinate System to Probe Transmitting

Coefficients in the Singly-Primed Coordinate System;

Rotation of a Singly-Primed Antenna into the

Orientation of a Doubly-Primed Antenna . . . . . . . . . . . .

6.5 On the Validity of Interchanging the Order of Summation of

$\mathrm{n} \leftrightarrow v$ Infinite Double Series .................... 67

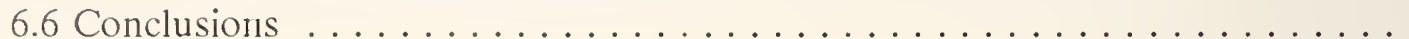

VII. REDUCTION OF THE TWO-ANTENNA COUPLING EQUATION TO THE MODAL-EXPANSION EQUATION WHEN ONE ANTENNA IS AN

ELEMENTARY DIPOLE . . . . . . . . . . . . . . . . . 75

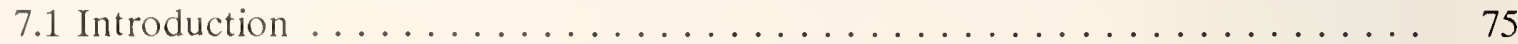

7.2 Modal Expansion Coefficients for an Elementary Dipole

Current Source ............................

7.3 Equality between the Transverse Radiated Field and the

Antenna-Dipole Coupling Equation: Receiving Electric

Dipole Case . . . . . . . . . . . . . . . . . . . . . . 78

7.4 Transmitting Electric Dipole Case . . . . . . . . . . . . . . 81

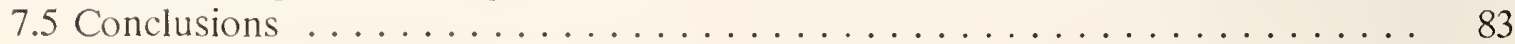

VIII. SPHERICAL SCANNING . . . . . . . . . . . . . . . . . . . 84

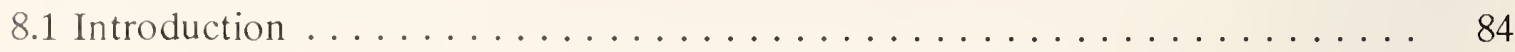

8.2 Probe-Correction Case Formulation for Spherical Scanning

Assuming a Transmitting Test Antenna and Receiving Probe . . . . . 85

8.2 .1 Basic Theoretical Development . . . . . . . . . . . . . . . . 85

8.2.2 Computation Algorithms for Discrete Data-Sampling Points . . . . . 89

8.2.3 An Efficient Computational Algorithm for Computing

the Far-Field Pattern from Near-Field Measurements . . . . . . 94

8.2.3.1 Computation of $\dot{W}_{n}^{m \mu} \ldots \ldots \ldots \ldots . \ldots \ldots$

8.2.3.2 Completion of the Near-Field to Far-Field

Computation Algorithm . . . . . . . . . . . . . . . . 101

8.3 Recapitulation of the Spherical-Scanning Algorithm's

Data-Processing Steps . . . . . . . . . . . . . . . . . . . . . . 108

8.4 A Modified Spherical-Scanning Algorithm for Radiation

Confincd to a Single Hemisphere . . . . . . . . . . . . . . . . . . . . 109 
IX. NUMERICAL COMPARISONS AND SIMULATIONS FOR VALIDATING

SPHERICAL-SCANNING COMPUTER CODE . . . . . . . . . . . . . . . 115

9.1 Introduction . . . . . . . . . . . . . . . . . . . . . . . 115

9.2 Numerical Self-Consistency Tests and Simulations . . . . . . . . . . . . . . . 116

9.3 Computational Aspects of Spherical-Scanning Data Processing . . . . . . . . . 122

9.3.1 Computational Limitations of the Probe-Translation Algorithm . . . . 122

9.3.2 Probe-Rotation Angles Corresponding to Measured-Data

Components ....................... 125

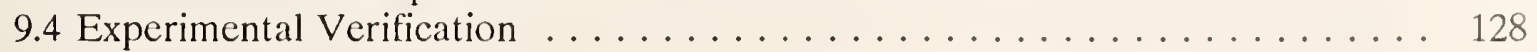

9.5 Conclusions .................................. 134

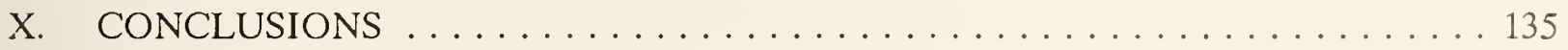

BIBLIOGRAPHY ........................... 138

APPENDIX A: EXPANSION OF AN INCIDENT PLANE-WAVE FIELD AS A SERIES OF VECTOR SPHERICAL-WAVE FUNCTIONS 


\section{FIGURES}

Figure

2-1. Notation for describing an antenna's spherical-wave source-scattering matrix (radial-distance $r$ is measured from 0 ). . . . . . . . . . . . . . 6

4-1. Coordinate system transformations. . . . . . . . . . . . . . . 27

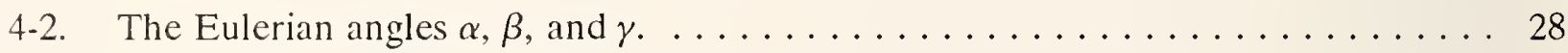

5-1. Two antenna transmission-system schematic. $\ldots \ldots \ldots \ldots \ldots \ldots \ldots$

5-2. Simplified configuration for studying a two-antenna transmission system

$$
\left(\phi^{\prime}=\phi^{\prime \prime}\right) \text {. }
$$

6-1. Rotation of a doubly primed antenna into the orientation of a singly primed antenna.

9-1. Test parameters. . . . . . . . . . . . . . . . . . . . . 130

9-2. Open-array near-field test setup. . . . . . . . . . . . . . . . . . . . . . 130

9-3. Near-field plot principal polarization. . . . . . . . . . . . . . . . 131

9-4. Transformed far-field plot principal polarization. . . . . . . . . . . . . 131

9-5. Sum elevation. ............................. 132

9-6. Sum azimuth. ............................. 132

9-7. Delta azimuth. ........................... 133

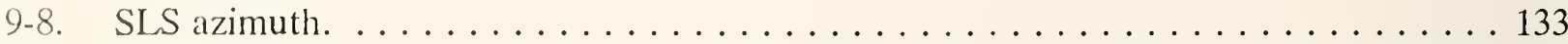




\section{ACKNOWLEDGMENTS}

I would especially like to acknowledge the encouragement and support of my $\mathrm{Ph} . \mathrm{D}$. thesis advisor, Dr. Samuel W. Maley, without which this work could not have been completed, and the steadfast encouragement of the second reader on my thesis committee, Dr. Norris $\mathrm{S}$.

Nahman. I should also like to thank my former instructors and Ph.D. committee members, Dr.

Frank S. Barnes, Dr. Carl T. A. Johnk, and Dr. Karl E. Gustafson for their encouragement and support over the years. Finally, I should like to thank my colleagues, in the Electromagnetic Fields Division of the National Institute of Standards and Technology, for their many contributions to my appreciation of the science we have been engaged in carrying out. 


\section{DEDICATION}

\section{TO MY FAMILY}

MY WIFE:

Betty L. Lewis

OUR CHILDREN:

\section{Pamela Dorothy Lewis}

Michelle Mary (Lewis) Grey

Kim Belinda (Cole) Gildow 


\title{
SPHERICAL-WAVE SOURCE-SCATTERING MATRIX ANALYSIS OF ANTENNAS AND ANTENNA-ANTENNA INTERACTIONS
}

\author{
Richard Lindsay Lewis \\ National Institute of Standards and Technology \\ Boulder, Colorado 80303
}

\begin{abstract}
Expressions are presented for describing incoming and outgoing fields about an antenna in terms of a series of exciting and emergent vector sphericalwave functions. The exciting and emergent fields around the antenna's exterior are related to the field in the antenna feed through a source-scattering matrix representation. Series of spherical-wave source-scattering matrix coefficients are used to express more conventional antenna parameters such as gain and rcceiving cross section. An overview of rotation and translation theorems for transforming vector spherical-wave functions between two distinct coordinate systems is given, followed by a general solution to the problem of expressing the coupling between two coupled antennas in terms of each antenna's spherical-wave source-scattering matrix representation. We go on to consider special results to substantiate our formulation, such as showing equivalence between the coupling equations for transmission in opposite directions when the antennas are reciprocal, showing uniform convergence of some series representations for antenna coupling and simultaneously obtaining a coordinate-system translation theorem for the dyadic Green's function, and showing that our two-antenna coupling equations reduce to expressions for the incident and emergent fields about a single antenna when the other antenna is an elementary dipole. Efficient probe-corrected spherical and hemispherical scanning algorithms are then developed for processing measured near-field data to obtain an antenna's far-field pattern. Finally, we describe a number of self-consistency tests and theoretical-data simulations that were developed to validate our spherical-scanning algorithm, and we describe an independent experimental verification.
\end{abstract}

Key words: antenna coupling; antenna measurements; dyadic Green's functions; modal expansion; multiple reflections; probe correction; reciprocity conditions; spherical scanning; scattering matrix

\section{CHAPTER I}

\section{INTRODUCTION}

This work presents a spherical-wave source-scattering-matrix analysis of antennas and antenna-antenna interactions analogous to Kern's treatment [25, 26] of plane-wave scattering matrices. ${ }^{1}$ In this regard, the representation for a single antenna is presented; then, conventional antcnna parameters are expressed in terms of this rcpresentation. A complctc

${ }^{1}$ The work presented in this Technical Note was published as the author's doctoral disscrtation at the University of Colorado, Boulder, Colorado, December 1992. 
formulation for the coupling between two antennas is presented. This formulation is subsequently used to develop an algorithm for near-field spherical scanning in the special case where multiple reflections between antennas can be neglected.

Kerns and Dayhoff [28] were the first to give a complete treatment using scattering matrices to describe antenna coupling. Wasylkiwskyj and Kahn [57] gave expressions for the mutual coupling between two minimum-scattering antennas using spherical-wave scattering matrices, which by definition ignored multiple reflections between the antennas. Yaghjian [60] presented a complete cylindrical-wave source-scattering-matrix analysis of the coupling between two antennas, in which the first-order multiple-reflection term was specifically presented. Yaghjian also coined the term, source-scattering matrix, to distinguish a scattering-matrix formulation, using cylindrical (or spherical) waves, in which the exciting spatial modes contain Bessel functions of the first kind as the radial-distance function. This particular radial-variable function enters into the formulation of the mutual-coupling problem in expressing the effect, on the cylindrical or spherical modes, of radially translating the coordinate origin.

We start our discussion by considering an antenna enclosed within a (fictitious) spherical surface and then go on to give expressions for the exciting and emergent spherical waves in the enclosed region. The totality of exciting waves constitutes the field incident on the spherical region from an external source, while the totality of emergent waves constitutes the field radiated from the spherical region. We complete our preliminary presentation by developing expressions for the conventional antenna parameters of gain and receiving cross-section in terms of sphericalwave source-scattering-matrix elements. At the same time, following Kerns [26], we derive spherical reciprocity relations, ultimately obtaining the classical relationship between receiving cross section and gain for a reciprocal antenna.

Next, we give an overview of the rotation [44] and translation [16] theorems that are needed for transforming the modal fields between two distinct coordinate systems. This is followed by a presentation of a general solution to the problem of two coupled antennas in terms of spherical-wave source-scattering-matrix coefficients. The resulting Liouville-Neumann series is very similar to the result obtained by Kerns [26] for the plane-wave scattering-matrix formulation. Following our development of the mutual-coupling relations between two antennas, we go on to consider easily verified special results to build confidence in our formulation. In this regard, we obtain two seemingly different transmission formulas, depending on the transmission direction, which are shown to be equivalent when the antennas are reciprocal. We also show that our expression for the coupling between two antennas reduces to an expression for the radiated electric field when one antenna is an elementary dipole.

We develop an efficient algorithm for implementing near-field spherical scanning, which is the process whereby measured near-field data, taken at discrete data points over the surface of a sphere enclosing the test antenna, are corrected for the effect of the measurement probe and then transformed to obtain the test antenna's far-field pattern. Also, since many antennas radiate negligibly toward their rear, we go on to develop a single-hemisphere scanning algorithm. 
This algorithm, in essence, breaks the spherical-scanning algorithm in half and processes both halves simultaneously, with a commensurate saving in computational effort.

A survey of the general subject of determining far-field patterns from near-field data is given in a paper by Johnson et al. [22], along with an extensive bibliography. An excellent survey of the previous work on probe-corrected near-field analysis is given by Kerns [26], along with the plane-wave near-field formulation. Treatments using cylindrical-coordinate near-field data to obtain a probe-corrected far-field pattern are given by Leach and Paris [33] and by Yaghjian [60]. Rahmat-Samii et al. [41] present a plane-polar analysis of the problem. The earliest spherical probe-compensated analysis is due to Jensen [21], who developed an expression for the signal received by the probe from the Lorentz reciprocity relations. Around the same time, Ludwig [39] independently developed a formulation for decoupling the electric-field vector's spherical expansion coefficients, using his analysis to obtain the far-field patterns for a circularly symmetric horn and a cassegrainian subreflector, thereby apparently becoming the first to make numerical calculations with a spherical near-field-to-far-field transformation.

The earliest practical spherical probe-corrected decoupling algorithm is due to Wacker [55], who introduced the use of a circularly symmetric probe to reduce the coupling complexity. Wacker also developed an expression for the received signal at the probe using a scatteringmatrix formulation and went on to develop a matrix formulation which made use of the fast Fourier transform (FFT) to obtain the far-field pattern of an antenna. In the current work, we take off from where Wacker left off to develop an improved, more efficient, spherical-scanning algorithm. In this regard, we recast the matrix formulation of Wacker into scalar-equation form and at the same time introduce some analytical simplifications designed to reduce the computational effort. In addition, we develop improvements on subsequent work by Larsen [31].

Intrinsically, the three principal near-field scanning techniques -- planar, cylindrical, and spherical -- are capable of producing equally accurate results provided the antenna under test is amenable to measurement by each method. The principal requirement for near-field scanning is to sample all of the significant radiation from the antenna as the probe traverses the scan surface. Put another way, the radiation pattern of the antenna under test dictates which scanning geometry is best. An antenna with well collimated radiation is best suited for planar scanning, an antenna with a fan beam in one dimension is best suited for cylindrical scanning, and an antenna whose radiation fans out in two dimensions requires spherical scanning. Although spherical scanning is the most general technique in that it is capable of handling any of these radiation patterns, it also involves the most complicated data processing algorithm and requires a specialized circularly symmetric probe. These disadvantages are somewhat offset by a simpler transport mechanism, in that the antenna under test rotates while the probe remains fixed. Since spherical scanning can accommodate antennas which are amenable to planar scanning, these two techniques can readily be compared against each other for selected antennas. Such tests have been carried out, with the result that excellent agreement has been obtained between the farfield patterns computed by these two techniques [59]. Another test of the validity of spherical scanning is to compare computed rcsults against the measured far field. Chaptcr IX of this work 
reports on a successful comparison between far-field measurements and results computed using our spherical-scanning algorithm.

In summary, then, this work develops the formulation for expressing conventional antenna parameters in terms of the spherical-wave source-scattering matrix, develops mutual-coupling relations between a pair of antennas, establishes equalities between expressions to build confidence in the mutual-coupling formulation, and develops efficient computational algorithms for both spherical and hemispherical scanning. The work is organized into ten chapters and one appendix, the present introduction being the first chapter.

In Chapter II, the functional notation for expressing the fields is introduced, and a modal expansion for the radiated field is given. Here, we also develop a series representation for the incident field due to a plane wave or an external dipole source.

In Chapter III, the sourcc-scattering matrix representation of the antenna is introduced, and we go on to develop expressions for reciprocity, gain, and receiving cross section.

In Chapter IV, we review functional transformations due to a coordinate-system transformation, and we present recursion relations that will be needed subsequently.

In Chapter V, we develop the mutual-coupling relations between two antennas, ending up with a Liouville-Neumann series for the "system two-port" whose terminals are the waveguide leads to the two antennas. ${ }^{2}$

Chapter VI establishes an equivalence between oppositely-directed transmission formulas for reciprocal antennas, expresses a reciprocal-antenna's receiving coefficients in terms of the same antenna's oppositely directed transmitting coefficients, and establishes the validity of certain interchanges, developed in Chapter V, in the order of summation of doubly infinite series.

In Chapter VII, equalities are established between the series representations for the electric field and the coupling equations for the special case where one antenna is an elementary dipole.

Chapter VIII presents a theoretical derivation of our spherical-scanning algorithm, and goes on to formulate a hemispherical-scanning algorithm. ${ }^{3}$

In Chapter IX, a number of self-consistency tests and theoretical-data simulations are developed for validating the sphcrical-scanning computer algorithm described in Chapter VIII. In addition, a number of analytical formulations related to these computational verifications are prescnted, along with an independent experimental verification.

Chapter X contains this work's summary and conclusions. Finally, in the appendix, we give mathematical details on the represcntation of an incident plane wave as an infinite series of vector spherical-wave functions.

${ }^{2}$ An abbreviatcd derivation of the rcsults presented in Chapter $\mathrm{V}$, using matrix algebra to condense the exposition, is given in [36]-A.

${ }^{3}$ An advanced version of the algorithm presented in Chapter VIII, using a numerically more cfficicnt process to carry out the ncar-ficld to far-field transformation, is given in [36]-B. 


\section{CHAPTER II}

\section{ELECTROMAGNETIC FIELD EXPANSIONS}

\subsection{Introduction}

The electromagnetic field in the source-free region exterior to a hypothetical spherical boundary enclosing an antenna will be represented here as an infinite series of vector sphericalwave functions, where each vector wave function satisfies the vector wave equation $\nabla \times \nabla \times \underline{F}-k^{2} \underline{F}=0$. The electromagnetic field can also be described as the linear combination of an outgoing field, propagating away from the spherical boundary, and an incident field, propagating toward the spherical boundary from external sources. If we assume an implied $\mathrm{e}^{-\mathrm{i} \omega t}$ time dependence, the outgoing field can be expressed as an infinite series of emergent vector spherical-wave functions each containing a spherical Hankel function of the first kind as the function of the radial $r$ coordinate, while the incident or incoming field can be expressed as an infinite scries of exciting vector spherical-wave functions each containing a spherical Bessel function of the first kind as the function of the radial $r$ coordinate. In addition to the clcctromagnetic field exterior to the antenna, we will want to characterize the field in the waveguide leads to the antenna in terms of incident and emergent modes.

Beyond characterizing general fields exterior to the hypothetical spherical boundary, we will dcvelop expressions for particular incoming fields, such as an incident plane wave or the field due to an cxternal dipole source. We will then go on to obtain an expression for the modalexpansion coefficients of the electromagnetic field in terms of the fields at the spherical boundary. Primarily, however, this chapter will introduce relevant notation for our further study of ficld representations.

\subsection{Representation of the Fields at the Terminal Surface $S_{0}$ and at the Spherical Surface $S$}

Let us consider the antenna system shown schematically in Figure 2-1. The (mathematical) tcrminal surface $S_{0}$ in the waveguide feed, together with the supplementary surface $S_{a}$, forms a closed surfacc enclosing the source or detector associated with the antenna. The surface $S_{a}$ coincides with the shielding. For simplicity, only a single waveguide-feed mode is assumed to propagatc in the waveguide fced. Conventional phasor-wave amplitudes $a_{0}$ and $b_{0}$ arc cmployed for the incident and emcrgent travcling-wave components at $S_{0}$. Thesc wave amplitudes are fully 


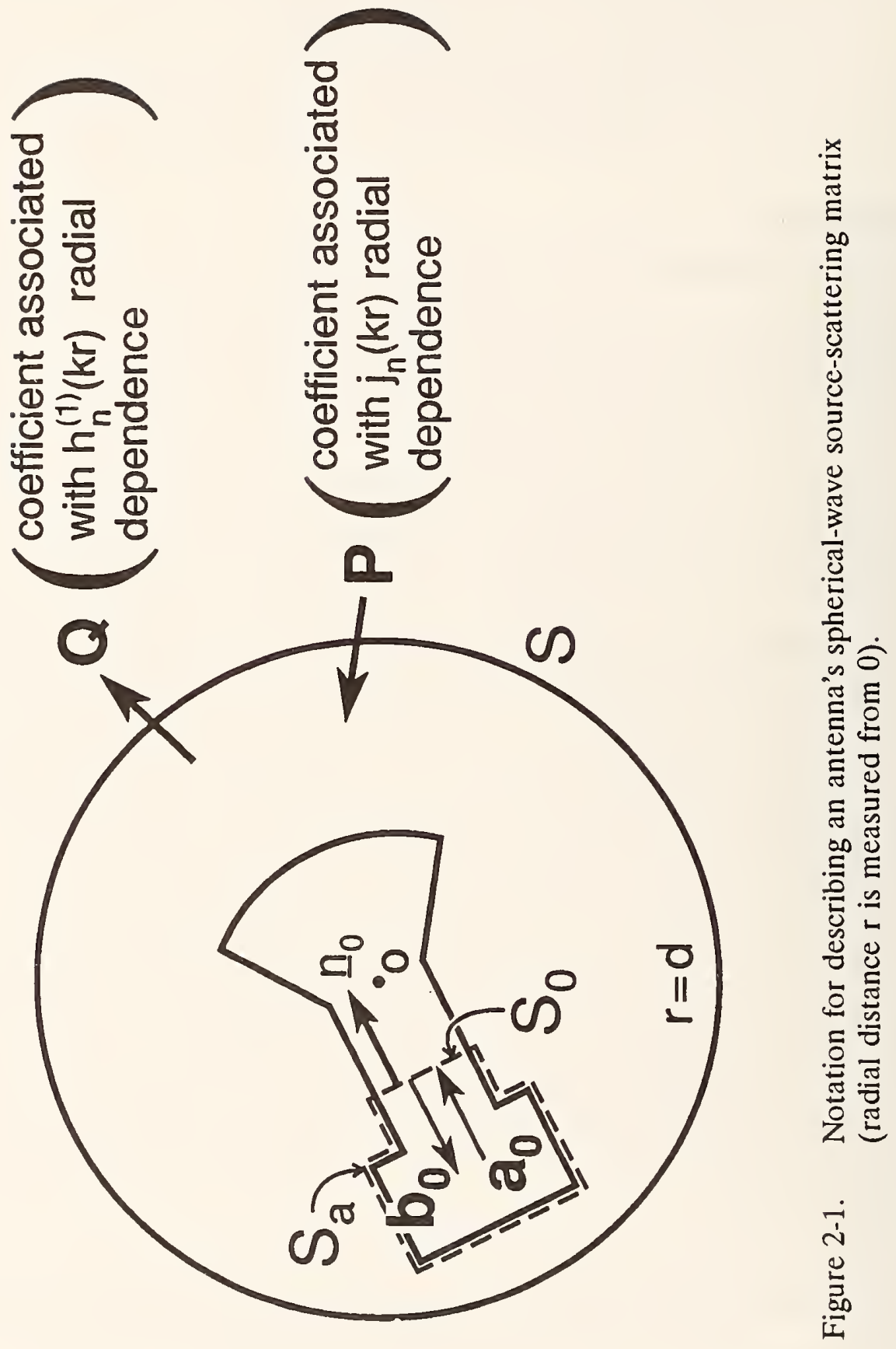


defined in Section 1.1 of Kerns' monograph [26]. There it is shown that the tangential electric and magnetic field components on $\mathrm{S}_{0}$ are

$$
\begin{aligned}
& \underline{E}_{0_{t}}=\left(a_{0}+b_{0}\right) \underline{e}_{0}(\underline{r}) \\
& \underline{H}_{0_{t}}=\hat{n}_{0}\left(a_{0}-b_{0}\right) \underline{h}_{0}(\underline{r}), \underline{r} \text { on } S_{0},
\end{aligned}
$$

where $\underline{\mathrm{e}}_{0}(\underline{\mathrm{r}})$ and $\underline{\mathrm{h}}_{0}(\underline{\mathrm{r}})$ are real basis functions for the mode involved, subject to the impedance normalization

$$
\underline{h}_{0}(\underline{r})=\hat{\eta}_{0}^{-1} \hat{\eta}_{w} \underline{n}_{0} \times \underline{e}_{0}(\underline{r})
$$

and to the power normalization

$$
\int_{S_{0}} \underline{e}_{0}(\underline{r}) \times \underline{h}_{0}(\underline{r}) \cdot \underline{n}_{0} d s=1 .
$$

Here $\underline{\mathrm{n}}_{0}$ is the unit normal vector on $\mathrm{S}_{0}$ pointing inward with respect to the antenna, $\hat{\eta}_{0}$ is the characteristic admittance, and $\hat{\eta}_{w}$ is the wave admittance for the mode involved. Additional details on this material are given by Kerns $[25,26]$, where it is also shown that the average power input at $S_{0}$ is

$$
P_{0}=\frac{1}{2} \operatorname{Re} \int_{S_{0}} \underline{E}_{0_{t}} \times \underline{H}_{0_{t}}^{*} \cdot \underline{n}_{0} d s=\frac{\hat{\eta}_{0}}{2}\left(\left|a_{0}\right|^{2}-\left|b_{0}\right|^{2}\right),
$$

where Re denotes that the real part is to be taken, the asterisk $\left({ }^{*}\right)$ denotes complex conjugate, and the paired vertical bars denote absolute values.

A spherical-coordinate system is chosen with unit vectors $\underline{a}_{r}, \underline{a}_{\theta}, \underline{a}_{\phi}$, and a (mathematical) sphere of radius $r=d$ centered about the origin of coordinates is then drawn so that the antenna and its feed lines are completely enclosed within the sphere by at least a few wavelengths. This spherical surface is depicted in Figure 2-1 as the surface S.

The electromagnetic field in the region exterior to the surface $S$ satisfies Maxwell's equations in the form

$$
\nabla \times \underline{E}=i \omega \mu \underline{H}, \quad \nabla \times \underline{H}=-i \omega \epsilon \underline{E},
$$

where $\mu, \epsilon$ are constants representing the permeability and permittivity of the medium, and $\exp (-\mathrm{i} \omega \mathrm{t})$ time dependence is assumed. As shown by Stratton [50, page 415] two sets of vector spherical-wave functions can be defined which are solutions of the vector wave equation

$$
\nabla \times \nabla \times \underline{F}-k^{2} \underline{F}=0
$$


where $\underline{F}$ represents either the electric or magnetic field vectors, and $k^{2}=\omega^{2} \mu \epsilon$. The two sets of vector spherical-wave functions that are finite at the origin of the coordinate system are

$$
\underline{M}_{m n}=\nabla \times\left(\psi_{m n} r\right) \text { and } \underline{N}_{m n}=\frac{1}{k} \nabla \times \underline{M}_{m n},
$$

where $\underline{r}=\underline{a}_{r}$ is the radial-vector position coordinate, and where $\psi_{m \mathbf{n}}$ is a particular solution of the scalar wave equation $\nabla^{2} \Psi_{m n}+k^{2} \Psi_{m n}=0$ in spherical coordinates that is finite at the origin. It can be written

$$
\Psi_{m n}=\sqrt{\frac{(n-m) !}{(n+m) !}} j_{n}(k r) P_{n}^{m}(\cos \theta) e^{i m \phi} .
$$

Here $\mathrm{r}, \theta, \phi$ describe the position coordinate of a point in space, $\mathrm{j}_{\mathrm{n}}(\mathrm{z})$ denotes the spherical Bessel function of the first kind of order $n$ and argument $z$, and $\mathrm{P}_{\mathrm{n}}^{\mathrm{m}}(\mathrm{x})$ denotes the associated Legendre function of degree $n$, order $m$, and argument $x$. The chosen definitions of these functions are (for instance, as in Abramowitz and Stegun [1])

$$
j_{n}(z)=\sqrt{\frac{\pi}{2 z}} J_{n+1 / 2}(z),
$$

where $J_{n+1 / 2}(z)$ is the cylindrical Bessel function of the first kind of argument $z$ and order $n+1 / 2$, and

$$
P_{n}^{m}(x)=\frac{(-)^{m}}{2^{n} n !}\left(1-x^{2}\right)^{\frac{m}{2}} \frac{d^{n+m}}{d x^{n+m}}\left(x^{2}-1\right)^{n} .
$$

The range on $\mathrm{m}$ in eq $(2.10)$ is $-\mathrm{n} \leq \mathrm{m} \leq \mathrm{n}$, where $\mathrm{n}$ is a nonnegative integer. Note that all negative order associated Legendre functions are each proportional to an associated Legendre function of positive order.

In our subsequent analysis, the spherical Hankel function of the first kind will also be required, which is related to the cylindrical Hankel function of the first kind by [1],

$$
h_{n}^{(1)}(z)=\sqrt{\frac{\pi}{2 z}} H_{n+1 / 2}^{(1)}(z) .
$$

The spherical Hankel function of the first kind owes its importance to the fact that it satisfies the Sommerfield radiation condition

$$
\lim _{r \rightarrow \infty} r\left(\frac{\partial}{\partial r}-i k\right) h_{n}^{(1)}(k r)=0 .
$$

Because of this property, the vector spherical-wave functions 


$$
\underline{M}_{m n}^{(1)}=\nabla \times\left(\underline{r} \Psi_{m n}^{(1)}\right) \text { and } \quad \underline{N}_{m n}^{(1)}=\frac{1}{k} \nabla \times \underline{M}_{m n}^{(1)}
$$

wherc

$$
\psi_{m n}^{(1)}=\sqrt{\frac{(n-m) !}{(n+m) !}} h_{n}^{(1)}(k r) P_{n}^{m}(\cos \theta) e^{i m \phi},
$$

represent emergent vector spherical modes.

We can now write a complete expression for the electromagnetic fields exterior to the spherical region $r=d$,

$$
\begin{aligned}
& \underline{E}(r, \theta, \phi)=\sum_{n=1}^{\infty} \sum_{m=-n}^{n}\left(Q^{1 m n} \underline{M}_{m n}^{(1)}+Q^{2 m n} \underline{N}_{m n}^{(1)}+P_{1 m n} \underline{M}_{m n}+P_{2 m n} \underline{N}_{m n}\right) \\
& \underline{H}(r, \theta, \phi)=-i \eta \sum_{n=1}^{\infty} \sum_{m=-n}^{n}\left(Q^{1 m n} \underline{N}_{m n}^{(1)}+Q^{2 m n} \underline{M}_{m n}^{(1)}+P_{1 m n} \underline{N}_{m n}+P_{2 m n} \underline{M}_{m n}\right),
\end{aligned}
$$

where $\eta=\sqrt{\frac{\epsilon}{\mu}}$. In the preceding expression, the Q's represent coefficients of the emergent modes. Similarly, the exciting-mode coefficients are represented by the P's. In Figure 2-1, the set of all the exciting-mode coefficients is symbolically represented by $\mathrm{P}$, while the set of all emergent-mode coefficients is represented by $Q$.

As indicated above, the satisfaction of the Sommerfield radiation condition suffices to assure emergent or outgoing mode behavior. In the next section we will examine a little more critically the assertion that the vector spherical-wave functions containing spherical Bessel functions of the first kind indeed make up a series solution for the representation of incident fields.

\subsection{Dyadic Green's Function Representations}

We wish to characterize electromagnetic fields due to sources external to the spherical region of interest. The exciting modes that form such incident fields have a radial-function dependence given by spherical Bessel functions of the first kind. Initially, we will ignore interactions between the exciting fields and objects within the spherical region of interest. Once the exciting electromagnetic fields are known, the presence of a scattering object can be fully accounted for by replacing the scattering object either with equivalent surface or volume currents, or with multipole sources within the spherical region which reproduce the scattered field. The total field is then obtained by invoking the superposition principle, which states that 
the total field is equal to the sum of the fields produced by all radiating sources in the absence of each other. That is, we determine the unperturbed electromagnetic fields produced by external sources in the absence of any scattering or transmitting objects in the spherical region of interest, and then add to this the electromagnetic fields due to equivalent surface or volume currents which replaced these scattering objects in order to obtain the total field.

The unperturbed electromagnetic fields which are excited external to the spherical region of interest will be characterized by a dyadic Green's function representation. Such a representation readily expresses a known current-source distribution in terms of vector sphericalwave functions. Alternately, we could obtain an equivalent representation from the orthogonality relations between vector spherical-wave functions, as is done in Appendix $A$ for the case of an incident plane-wave field. Two parallel treatments for the case of incident plane-wave excitation emerge from pursuing both avenues. These parallel treatments serve to confirm our final result, which differs slightly from a previously reported expression [9] (see Appendix A). Our final result is subsequently used to characterize the receiving cross section of an antenna located within the spherical region of interest and to demonstrate an equivalence between our expression for the electric field and our yet to be obtained expression for the coupling between two antennas in the special case where one antenna is an elementary dipole.

We shall characterize an external source by a vector current-density function of position, $\underline{J}^{\prime \prime} \equiv \underline{J}\left(\underline{r}^{\prime \prime}\right)=\underline{J}\left(r^{\prime \prime}, \theta^{\prime \prime}, \phi^{\prime \prime}\right)$, which has nonzero values only in a region of space external to the spherical region of interest. Here, doubly primed coordinates designate coordinates of the delta-function source of the Green's function, while unprimed coordinates designate the position coordinates of the field point or location of the observer. Attaching double primes to a scalar or vector function signifies that its implicit position variables are source coordinates (that is, defined with respect to the doubly primed coordinate system), whereas the lack of either primes or explicit position variables signifies that unprimed or observation-point coordinates are implied. In the present application, the origin and orientation of the doubly primed coordinate system is chosen coincident with the origin and orientation of the unprimed coordinate system. However, in a subsequent chapter we shall make use of doubly primed coordinates in a different manner, such that doubly primed coordinates designate a coordinate system that is displaced and rotated with respect to the unprimed coordinate system. Since the present application does not extend beyond this chapter, there should be no confusion between these two different uses.

The electric field in the spherical region of interest, in the absence of any scattering objects within that region, is given by Tai $[51,52]$ as 


$$
\underline{E}(\underline{r})=i \omega \mu \iiint \underline{\underline{G}}\left(\underline{r} \mid \underline{r}^{\prime \prime}\right) \cdot \underline{J}\left(\underline{r}^{\prime \prime}\right) d v^{\prime \prime},
$$

whcre $\underline{r}$ denotes the position vector in the unprimed coordinate system, $\underline{r}^{\prime \prime}$ the position vector in the doubly primed coordinate system, $\mathrm{dv}^{\prime \prime}$ is the volume element of integration over the doubly primed coordinates, and $\underline{\underline{G}}\left(\boldsymbol{r} \mid \underline{r}^{\prime}\right)$ is the dyadic electric Green's function which, by definition, satisfies the dyadic differential equation,

$$
\nabla \times \nabla \times \underline{\underline{G}}\left(\underline{r} \mid \underline{r}^{\prime \prime}\right)-k^{2} \underline{\underline{G}}\left(\underline{r} \mid \underline{r}^{\prime \prime}\right)=\underline{\underline{I}} \delta\left(\underline{r}-\underline{r}^{\prime \prime}\right)
$$

and the radiation condition.

$$
\lim _{r \rightarrow \infty} r\left[\nabla \times \underline{\underline{G}}\left(\underline{r} \mid \underline{r}^{\prime \prime}\right)-i k \underline{r} \times \underline{\underline{G}}\left(\underline{r} \mid \underline{r}^{\prime \prime}\right)\right]=0 .
$$

In eq (2.17), $\underline{\underline{I}}=\underline{a}_{r} \underline{a}_{r}+\underline{a}_{\theta} \underline{a}_{\theta}+\underline{a}_{\phi} \underline{a}_{\phi}$ is the unit dyadic while $\delta\left(r-r^{\prime \prime}\right)$ denotes a threedimensional delta function that is characterized by

$$
\iiint f\left(\underline{r}^{\prime \prime}\right) \delta\left(\underline{r}-\underline{r}^{\prime \prime}\right) d v^{\prime \prime}=f(\underline{r})
$$

Since the evaluation of eq (2.16) is only of interest for $\underline{r}$ values that are exterior to the region containing the current density vector $\underline{I}^{\prime \prime}$, we do not need to worry about any singularities of the dyadic Green's function [52] in the representation of $\underline{\underline{G}}\left(r \mid r^{\prime \prime}\right)$. Consequently, we can use the results in [51] after making some slight modifications to account for the fact that the vector spherical-wave functions, as defined by Tai, are defined with respect to scalar eigenfunctions in which the complex exponential $e^{\mathrm{i} m \phi}$ is replaced by $\cos m \phi$ and $\sin m \phi$. The subscripts "e" and "o," denoting even and odd, are correspondingly appended to the eigenfunctions in Tai's representation. Accordingly, in order to use the definitions eq (2.7) and (2.13) for the vector spherical-wave functions, we could parallel exactly the development of Tai [51, section 39], using the Ohm-Rayleigh method to derive from basic principles an expression for $\underline{\underline{G}}\left(\left.\underline{r}\right|^{\prime \prime}\right)$ in terms of these definitions. An alternative method of arriving at the same result is to relate Tai's even and odd vector spherical-wave functions to the complex-exponential vector spherical-wave functions defined in eq (2.7); then these expressions can be substituted into Tai's final expression [51, eq (39.18)]. The presentation will be the latter alternative, although independently carrying out an analysis based on the first option guided the way.

Denoting Tai's expressions for the $\underline{\mathrm{M}}$ vector spherical-wave functions by

$$
{\underline{M_{e}}}_{e_{m}} \underline{M}_{e_{m}}^{(1)^{\prime \prime}}=\underline{M}_{e m n} \underline{M}_{e m n}^{(1)^{\prime \prime}}+\underline{M}_{o m n} \underline{M}_{o m n}^{(1)^{\prime \prime}}
$$


and using a similar expression to denote the $\underline{\mathrm{N}}$ functions, Tai's final result is

$$
\begin{aligned}
& \underline{\underline{G}}\left(\underline{r} \mid \underline{r}^{\prime \prime}\right)=\frac{i k}{4 \pi} \sum_{n=1}^{\infty} \sum_{m=0}^{n}\left(2-\delta_{m 0}\right) \frac{2 n+1}{n(n+1)} \frac{(n-m) !}{(n+m) !}
\end{aligned}
$$

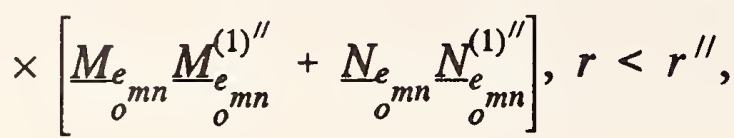

where $\delta_{m 0}=\left\{\begin{array}{ll}0, & m \neq 0 \\ 1, & m=0\end{array}\right.$. The unprimed $\underline{M}$ and $\underline{N}$ denote functions of $\underline{\mathrm{r}}$, while the doubly primed $\underline{M}^{\prime \prime}$ and $\underline{N}^{\prime \prime}$ denote functions of $\underline{r}^{\prime \prime}$. As before, a (1) superscript on the vector sphericalwave functions designates the spherical Hankel function of the first kind for the radial functions, while the absence of such a superscript designates the spherical Bessel function of the first kind for the radial function. Note the restriction in eq (2.21) that $r<r^{\prime \prime}$. For $r>r^{\prime \prime}$, the $\underline{M}$ and $\underline{N}$ functions containing the Bessel function become functions of the doubly primed coordinates, while the $\underline{\mathrm{M}}$ and $\underline{\mathrm{N}}$ functions containing the Hankel function become functions of the unprimed coordinates. The relationship between the complex-exponential representation for the vector spherical-wave functions and the even/odd representation is, for the $\underline{M}$ functions,

$$
\begin{gathered}
\underline{M}_{m n}=\sqrt{\frac{(n-m) !}{(n+m) !}\left[\underline{M}_{e m n}+i \underline{M}_{o m n}\right]} \\
\underline{M}_{-m, n}=(-)^{m} \sqrt{\frac{(n-m) !}{(n+m) !}}\left[\underline{M}_{e m n}-i \underline{M}_{o m n}\right]
\end{gathered}
$$

and similarly for the $\underline{\mathrm{N}}$ functions. Substituting expressions for both $\underline{\mathrm{M}}_{\mathrm{mn}}$ and $\underline{\mathrm{N}}_{\mathrm{m} n}$ corresponding to eq (2.22) into eq (2.21) and collecting terms yields the desired expression for the dyadic Green's function expressed in terms of the vector spherical-wave functions defined in section 2.1,

$$
\underline{\underline{G}}\left(\underline{r} \mid \underline{r}^{\prime \prime}\right)=\frac{i k}{4 \pi} \sum_{n=1}^{\infty} \sum_{m=-n}^{n}(-)^{m} \frac{2 n+1}{n(n+1)}\left[\underline{M}_{m n} \underline{M}_{-m n}^{(1)^{\prime \prime}}+\underline{N}_{m n} \underline{M}_{-m n}^{(1)^{\prime \prime}}\right], r<r^{\prime \prime} \text {. }
$$

We shall use eq (2.23) in conjunction with eq (2.16) to obtain an expression for the electric-field vector incident at the boundary of the spherical region of interest for the specific case of excitation by an elementary-dipole source. Before proceeding further, we need to express a complex-conjugate relation between those vector spherical-wave functions whose radial dependence function is a Bessel function. Thus, from eqs (2.7) and (2.8), in conjunction with the 
expression [3] relating negative-order associated Legendre functions to positive-order associated Legendre functions, we obtain

$$
\underline{M}_{m n}^{*}=(-)^{m} \underline{M}_{-m, n}
$$

and

$$
\underline{N}_{m n}^{*}=(-)^{m} \underline{N}_{-m, n},
$$

where the asterisk denotes the complex conjugate. This leads immediately to an alternative expression for the dyadic Green's function,

$$
\underline{\underline{G}}\left(\underline{r} \mid \underline{r}^{\prime \prime}\right)=\frac{i k}{4 \pi} \sum_{n=1}^{\infty} \sum_{m=-n}^{n} \frac{2 n+1}{n(n+1)}\left[\underline{M}_{m n}^{*} \underline{M}_{m n}^{(1)^{\prime \prime}}+\underline{N}_{m n}^{*} \underline{N}_{m n}^{(1)}\right], r<r^{\prime \prime} .
$$

We now wish to substitute eq (2.23) into (2.16) and evaluate the resulting integral for the dipole source,

$$
\underline{J}^{\prime \prime}=\vec{p}^{\prime \prime} \frac{\delta\left(r^{\prime \prime}-r_{0}\right) \delta\left(\theta^{\prime \prime}-\theta_{0}\right) \delta\left(\phi^{\prime \prime}-\phi_{0}\right)}{r_{0} \sin \theta^{\prime \prime}},
$$

where $\vec{p}^{\prime \prime}$ is the dipole current moment,

$$
\vec{p}^{\prime \prime}=\operatorname{Id} l\left(\underline{a}_{\theta^{\prime \prime}} \cos \gamma+\underline{a}_{\phi^{\prime \prime}} \sin \gamma\right) .
$$

In eq (2.26), $\mathrm{r}_{0}, \theta_{0}, \phi_{0}$ denote the position of the dipole source with respect to either the doubly primed or the unprimed coordinate systems. The angle $\gamma$, on the other hand, designates the orientation angle for the dipole source. Upon carrying out the indicated integration there results,

$$
\begin{gathered}
\underline{E}(\underline{r})=-\frac{\omega \mu_{0} k}{4 \pi} \sum_{n=1}^{\infty} \sum_{m=-n}^{n} \frac{2 n+1}{n(n+1)} \vec{p} \cdot\left[h_{n}^{(1)}\left(k r_{0}\right) \underline{G}_{n}^{1 m *}\left(\theta_{0}, \phi_{0}\right) \underline{M}_{m n}\right. \\
\left.+\frac{1}{k r_{0}} \frac{\partial}{\partial r_{0}}\left(r_{0} h_{n}^{(1)}\left(k r_{0}\right)\right) \underline{G}_{n}^{2 m *}\left(\theta_{0}, \phi_{0}\right) \underline{N}_{m n}\right]
\end{gathered}
$$

where

$$
\vec{p}=I d \ell\left(\underline{a}_{\theta_{0}} \cos \gamma+\underline{a}_{\phi_{0}} \sin \gamma\right)
$$

is the new dipole current moment. In eq (2.27) we have introduced a special notation for the transverse parts of the vector spherical-wave functions with the radial function factored out. The definitions of these factored transverse vector spherical-wave functions arc

$$
\underline{M}_{m n}^{(1)}=h_{n}^{(1)}(k r) \underline{G}_{n}^{1 m}(\theta, \phi)
$$


and

$$
\underline{N}_{m n_{t}}^{(1)} \equiv \underline{N}_{m n}^{(1)}-\underline{a}_{r}\left(\underline{N}_{m n}^{(1)} \cdot \underline{a}_{r}\right)=\frac{1}{k r} \frac{\partial}{\partial r}\left(r h_{n}^{(1)}(k r)\right) \underline{G}_{n}^{2 m}(\theta, \phi) .
$$

From these definitions and the complex-conjugate relations eq (2.24) it immediately follows that

$$
\underline{G}_{n}^{1 m *}(\theta, \phi)=(-)^{m} \underline{G}_{n}^{1,-m}(\theta, \phi)
$$

and

$$
\underline{G}_{n}^{2 m *}(\theta, \phi)=(-)^{m} \underline{G}_{n}^{2,-m}(\theta, \phi) .
$$

The notation used here for the transverse parts of the vector spherical-wave functions corresponds with that used by Wacker [56], whereas Tai [51] used lower case $\underline{\underline{m}}_{e^{m n}}$ and $\underline{a}_{r} \times \underline{m}_{0^{m n}}$ in place of $\underline{G}_{n}^{1 m}$ and $\underline{G}_{n}^{2 m}$. Specific expressions are

$$
\underline{G}_{n}^{1 m}(\theta, \phi)=\sqrt{\frac{(n-m) !}{(n+m) !}}\left[\frac{i m}{\sin \theta} P_{n}^{m}(\cos \theta) \underline{a}_{\theta}-\frac{\partial}{\partial \theta} P_{n}^{m}(\cos \theta) \underline{a}_{\phi}\right] e^{i m \phi}
$$

and

$$
\underline{G}_{n}^{2 m}(\theta, \phi)=\underline{a}_{r} \times \underline{G}_{n}^{1 / n}(\theta, \phi)
$$

Next, we consider the asymptotic expansion of the spherical Hankel functions. From Abramowitz and Stegun [1], we have for $\mathrm{kr}>>1$,

$$
h_{n}^{(1)}(k r) \sim i^{-n-1} \frac{e^{i k r}}{k r} ; \quad \frac{1}{k r} \frac{\partial}{\partial r}\left(r h_{n}^{(1)}(k r)\right) \sim i^{-n} \frac{e^{i k r}}{k r} .
$$

Considerably more detail on the second of these expressions is given by Jensen [21].

Consequently, when the dipole source is considerably removed from the spherical region of interest, eq (2.27) becomes

$$
\begin{array}{r}
\underline{E}(\underline{r}) \sim-\frac{\omega \mu_{0}}{4 \pi r_{0}} e^{i k r_{0}} \sum_{n=1}^{\infty} \sum_{m=-n}^{n} \frac{2 n+1}{n(n+1)} i^{-n}\left[-i \underline{M}_{m n} \underline{G}_{n}^{1 m *}\left(\theta_{0}, \phi_{0}\right)\right. \\
\left.+\underline{N}_{m n} \underline{G}_{n}^{2 m *}\left(\theta_{0}, \phi_{0}\right)\right] \cdot \vec{p} .
\end{array}
$$

In the limit, as $r_{0}$ approaches infinity, the primary field of the dipole source degenerates into a plane wave. Thus, defining

$$
\mathscr{E}_{0}=-\frac{i \omega \mu_{0} I d \ell}{4 \pi r_{0}} e^{i k r_{0}}
$$

we obtain the electric field in the spherical region of interest due to an incident plane wave 


$$
\begin{aligned}
\underline{E}(\underline{r})=\mathscr{E}_{0} \sum_{n=1}^{\infty} & \sum_{m=-n}^{n} \frac{2 n+1}{n(n+1)} i^{-n-1}\left[-i \underline{M}_{m n} \underline{G}_{n}^{1 m *}\left(\theta_{0}, \phi_{0}\right)\right. \\
& \left.+\underline{N}_{m n} \underline{G}_{n}^{2 m *}\left(\theta_{0}, \phi_{0}\right)\right] \cdot \vec{q},
\end{aligned}
$$

where

$$
\vec{q}=\underline{a}_{\theta_{0}} \cos \gamma+\underline{a}_{\phi_{0}} \sin \gamma
$$

specifies the polarization of the incident plane wave. The alternative derivation in Appendix A for characterizing an incident plane-wave field, given by eqs (A.7), (A.13), and (A.19), agrees completely with (2.36).

In eq (2.36) we have characterized an incident plane wave in terms of vector sphericalwave functions containing spherical Bessel functions of the first kind. Thus, the coefficients of $\underline{\mathrm{M}}_{\mathrm{mn}}$ and $\underline{\mathrm{N}}_{\mathrm{nIn}}$ in eq (2.36) respectively correspond to $\mathrm{P}_{1 \mathrm{mn}}$ and $\mathrm{P}_{2 \mathrm{mn}}$ in eq (2.15) when the incident field is a plane wave. The generalization of this result, in which any external source is found to just produce vector spherical-wave functions containing spherical Bessel functions of the first kind, follows directly from eqs (2.16) and (2.25). On the other hand, any scattering object within the spherical region of interest is represented by equivalent surface current sources which exclusively produce emergent modes described by vector spherical-wave functions containing spherical Hankel functions of the first kind. Consequently, we have established the validity of our characterization (2.15) of exciting and emergent wave behavior.

\subsection{Orthogonality Relations for the Vector Spherical-Wave Functions}

The orthogonality relations satisfied by the vector spherical-wave functions can be presented in a relatively simple fashion using the definitions of Section 2.2. Thus, the expressions in either Stratton [50] or Tai [51] reduce to

$$
\begin{gathered}
\int_{0}^{2 \pi} d \phi \int_{0}^{\pi} \sin \theta d \theta \underline{N}_{m n} \cdot \underline{N}_{p q}^{*}=4 \pi \frac{n(n+1)}{(2 n+1)^{2}}\left\{(n+1)\left[j_{n-1}(k r)\right]^{2}\right. \\
\left.+n\left[j_{n+1}(k r)\right]^{2}\right\} \delta_{m p} \delta_{n q} \\
\int_{0}^{2 \pi} d \phi \int_{0}^{\pi} \sin \theta d \theta \underline{M}_{m n} \cdot \underline{M}_{p q}^{*}=4 \pi \frac{n(n+1)}{2 n+1}\left[j_{n}(k r)\right]^{2} \delta_{m p} \delta_{n q}
\end{gathered}
$$




$$
\int_{0}^{2 \pi} d \phi \int_{0}^{\pi} \sin \theta d \theta \underline{M}_{m n} \cdot \underline{N}_{p q}^{*}=0
$$

where $\delta_{\mathrm{mp}}=1$ for $\mathrm{m}=\mathrm{p}$ and is zero otherwise. The applieation of these orthogonality relations to determine the modal-expansion coeffieients for an incident plane-wave field is presented in Appendix A, which provides us with an alternative derivation of eq (2.36) for a verification of that result.

The orthogonality relations between the transverse eomponents of the veetor sphericalwave funetions are useful for determining the modal-expansion coeffieients when just the transverse electrie field is known on the spherieal surface $S$ (see Figure 2-1). These relationships are

$$
\int_{0}^{2 \pi} d \phi \int_{0}^{\pi} \sin \theta d \theta \underline{G}_{n}^{s m}(\theta, \phi) \cdot \underline{G}_{q}^{\sigma p *}(\theta, \phi)=4 \pi \frac{n(n+1)}{2 n+1} \delta_{s \sigma} \delta_{m p} \delta_{n q},
$$

where s, $\sigma=1$ or 2 . Using eq (2.32), we can write an alternate version of this result,

$$
\begin{gathered}
\int_{0}^{2 \pi} d \phi \int_{0}^{\pi} \sin \theta d \theta \underline{G}_{n}^{s m}(\theta, \phi) \times \underline{G}_{q}^{\sigma+1, p}(\theta, \phi) \cdot \underline{a}_{r} \\
=(-)^{s+1} 4 \pi \frac{n(n+1)}{2 n+1}(-)^{m} \delta_{s \sigma} \delta_{m,-p} \delta_{n q},
\end{gathered}
$$

where the eonvention is adopted that the quantity $(\sigma+1)$ is eyclie on the integers 1,2 . That is $\underline{G}_{q}^{3, p} \equiv \underline{G}_{q}^{1, p}$.

In order to obtain more compact expressions and notation, we now present some alternate formulations of the eleetromagnetie-field expression (2.15). First, we introduce a notation due to Jensen [21]

$$
\begin{gathered}
R_{1 n}^{(1)}(r)=h_{n}^{(1)}(k r), \quad R_{2 n}^{(1)}(r)=\frac{1}{k r} \frac{\partial}{\partial r}\left(r h_{n}^{(1)}(k r)\right) \\
R_{1 n}(r)=j_{n}(k r), \quad R_{2 n}(r)=\frac{1}{k r} \frac{\partial}{\partial r}\left(r j_{n}(k r)\right) .
\end{gathered}
$$

Using these expressions, we ean write for the transverse part of the electric field,

$$
\underline{E}_{t}(\underline{r})=\sum_{s=1}^{2} \sum_{n=1}^{\infty} \sum_{m=-n}^{n}\left[Q^{s m n} R_{s n}^{(1)}(r)+P_{s m n} R_{s n}(r)\right] \underline{G}_{n}^{s m}(\theta, \phi),
$$

whilc a similar expression applies for the transverse part of the magnetic field. Now using the detcrminant relation [21, p. 118], 


$$
\left|\begin{array}{lc}
R_{s n}^{(1)}(r) & R_{s n}(r) \\
R_{s+1, n}^{(1)}(r) & R_{s+1, n}(r)
\end{array}\right|=(-)^{s} \frac{i}{(k r)^{2}}
$$

and the orthogonality relation eq (2.39), we can obtain expressions for the modal-expansion coefficients in terms of integrals of the tangential electric and magnetic field components over the surface of the sphere S. Thus, the following two-by-two matrix equation results,

$$
\begin{aligned}
& {\left[\begin{array}{l}
Q^{s m n} \\
P_{s m n}
\end{array}\right]=i \frac{(k r)^{2}}{4 \pi}(-)^{s+1} \frac{2 n+1}{n(n+1)}\left[\begin{array}{cc}
R_{s+1, n}(d) & -R_{s n}(d) \\
-R_{s+1, n}^{(1)}(d) & R_{s n}^{(1)}(d)
\end{array}\right]} \\
& \times\left[\begin{array}{l}
\int_{0}^{2 \pi} d \phi \int_{0}^{\pi} \sin \theta d \theta \underline{E}_{t}(r) \cdot \underline{G}_{n}^{s m *}(\theta, \phi) \\
\frac{i}{\eta} \int_{0}^{2 \pi} d \phi \int_{0}^{\pi} \sin \theta d \theta \underline{H}_{t}(r) \cdot \underline{G}_{n}^{s+1, m *}(\theta, \phi)
\end{array}\right]_{(r=d)},
\end{aligned}
$$

In eq (2.44), the transverse electric and magnetic field components $\underline{E}_{t}$ and $\underline{H}_{t}$ are evaluated at the radial distance $\mathrm{r}=\mathrm{d}$.

Finally, we present some alternate expressions that are equivalent to the electromagneticfield expressions eq (2.15) but which use a shorthand notation for the vector spherical-wave functions. This shorthand notation was previously introduced by both Jensen [21] and Wacker [56]. Thus, we define

$$
\underline{F}_{1 m n}^{(1)}=\underline{M}_{m n}^{(1)}, \quad \underline{F}_{2 m n}^{(1)}=\underline{N}_{m n}^{(1)}
$$

The placement of the subscripts and superscripts in eq (2.45) corresponds with Jensen's notation, while use of upper case F's corresponds with Wacker's notation. Adopting the notation of eq (2.45) we can rewrite eq $(2.15)$ as

$$
\begin{gathered}
\underline{E}(\underline{r})=\sum_{s=1}^{2} \sum_{n=1}^{\infty} \sum_{m=-n}^{n}\left[Q^{s m n} \underline{F}_{s m n}^{(1)}+P_{s m n} \underline{F}_{s m n}\right] \\
\underline{H}(r)=-i \eta \sum_{s=1}^{2} \sum_{n=1}^{\infty} \sum_{m=-n}^{n}\left[Q^{s m n} \underline{F}_{s+1, m n}^{(1)}+P_{s m n} \underline{F}_{s+1, m n}\right],
\end{gathered}
$$

where $\mathrm{s}+1$ is cyclic on the integers 1,2 . Thus, $\underline{F}_{3 m n} \equiv \underline{F}_{1 m n}$. 


\subsection{Conclusions}

In this chapter, we have introduced notation for expressing the fields on a terminal surface $S_{\mathrm{o}}$ in an antenna's waveguide feed (eq (2.1)), and we have introduced notation for expressing the fields in the source-free region beyond a bounding spherical surface S enclosing an antenna (eq (2.15)). We have then gone on to introduce a dyadic Green's function representation for the field incident on a spherical boundary $S$ from sources outside this boundary, and in particular we have characterized the incident field due to an external dipole source (eq (2.27)) and a propagating plane wave (eq (2.36)), where the latter expression is verified by an alternative derivation presented in Appendix A. At the same time, we have introduced relationships between positive and negative $\mathrm{m}$-index vector spherical-wave functions (eq (2.24)), along with asymptotic results for spherical Hankel functions and their derivatives when the functional argument is large (eq (2.33)). These results will be used in our subsequent development of an algorithm for spherical scanning. We concluded this chapter with a section on the orthogonality relations between vector spherical-wave functions upon integration over the surface of a sphere (eq (2.38)), in which we developed expressions for the modal-expansion coefficients of the electromagnetic field in terms of electric and magnetic field surface integrals over the spherical boundary $\mathrm{S}$ (eq (2.44)). In the next chapter, we will use the notation developed here to relate fields in the waveguide feed to transmitted and received antenna fields, and subsequently we will use our notational development to characterize antenna-antenna interactions. 


\section{CHAPTER III}

\section{SPHERICAL-WAVE SOURCE-SCATTERING MATRIX REPRESENTATION OF AN ANTENNA}

\subsection{Introduction}

The earliest presentation of a plane-wave scattering-matrix formulation to describe the connection between a traveling-wave mode propagating along the waveguide feed to an antenna and the plane-wave modal expansion for the fields exterior to that antenna appears to be that of Kerns and Dayhoff [28]. The very first scattering-matrix representation undoubtedly is due to Dicke [40]. Here, we present a source-scattering matrix formulation to represent an antenna with respect to emergent and exciting spherical modes as described in the previous chapter. The terminology, "source-scattering matrix," was coined by Yaghjian [60] in his formulation of cylindrical scanning to distinguish his scattering-matrix representation from Dicke's.

\subsection{Formulation of the Source-Scattering Matrix Representation}

Our source-scattering matrix equation, relating the incident and emergent traveling- wave components $a_{o}$ and $b_{o}$ at the terminal surface $S_{o}$ in the waveguide feed to the exciting and emergent spatial-mode components $\mathrm{P}$ and $\mathrm{Q}$ at the spherical surface $\mathrm{S}$ (refer to Figure 2-1), is given by

$$
\left[\begin{array}{l}
b_{0} \\
Q
\end{array}\right]=\left[\begin{array}{ll}
\Gamma & R \\
T & S
\end{array}\right]\left[\begin{array}{l}
a_{0} \\
P
\end{array}\right] .
$$

Here, $\Gamma$ is the input reflection coefficient of the antenna, and $T, R$, and $S$ represent all of its transmitting, receiving, and scattering properties. $\Gamma$ is a complex number; $Q, T$, and $P$ are infinite-dimension column matrices; $\mathrm{R}$ is an infinite row matrix; and $\mathrm{S}$ is an infinite square matrix.

The preceding matrix equation can be explicitly written out to yield

$$
\begin{aligned}
b_{0} & =\Gamma a_{0}+\sum_{s n m} R_{s m n} P_{s m n}, \\
Q^{s m n} & =T^{s m n} a_{0}+\sum_{\sigma \nu \mu} S_{\sigma \mu \nu}^{s m n} P_{\sigma \mu \nu},
\end{aligned}
$$

Here, the notation is adopted, for instance, that

$$
\sum_{\sigma \nu \mu}=\sum_{\sigma=1}^{2} \sum_{\nu=1}^{\infty} \sum_{\mu=-v}^{v} .
$$


This completes the antenna's spherical-wave source-scattering matrix definition. In order to appreciate the potential of this formulation, we will follow Kerns' treatment $[25,26]$ of the plane-wave counterpart and present some of the more familiar and conventional quantities of antenna theory in terms of these spherical-wave source-scattering matrix parameters.

\subsection{Reciprocity Relations}

The material in this section parallels closely Kerns' presentation of reciprocity relationships [26, chap. II, sec. 1.5 and appendix A; or 25, sec. 1.5 and appendix A]. Although the material presented here omits the concepts of "adjoint-reciprocity" developed by Kerns, the extension required to admit this more general treatment is straightforward. Consequently, for the purposes of this section, ordinary reciprocity in the sense defined in chapter II, appendix A of Kerns [25] is assumed for the antenna under consideration. However, unless specifically noted, subsequent sections Chapter III, as well as other chapters, will not make any assumption regarding antenna reciprocity. ${ }^{1}$

Referring to Figure 2-1 of Section 2.2, we consider a volume integral throughout the region $\mathrm{V}$ bounded by the surfaces $\mathrm{S}$ and $\mathrm{S}_{\mathrm{o}}+\mathrm{S}_{\mathrm{a}}$. The divergence theorem gives the result,

$$
\iiint_{\mathrm{v}} \nabla \cdot \underline{L} d v=\iint_{S} \underline{\underline{L}} \cdot \underline{a}_{r} d s-\iint_{S_{0}} \underline{L} \cdot \underline{n}_{0} d s
$$

where we have assumed that the surface integral over $\mathrm{S}_{\mathrm{a}}$ equals zero, which it must for ${ }^{2}$

$$
\underline{L}=\underline{E}^{\prime} \times \underline{H}^{\prime \prime}-\underline{E}^{\prime \prime} \times \underline{H}^{\prime},
$$

where $\underline{E}^{\prime}, \underline{H}^{\prime}$ and $\underline{E}^{\prime \prime}, \underline{H}^{\prime \prime}$ are two independent electromagnetic fields that can exist in V. Now by assumption the volume $\mathrm{V}$ is source free; hence [12, chapter 4$]$ the Lorentz reciprocity relation applies, or $\nabla \cdot \underline{L}=0$ throughout $V$. Therefore, it readily follows from eqs (2.3), (2.40), (2.42), and (2.44) that eq $(3.5)$ reduces to

$$
\hat{\eta}_{0}\left(a_{0}^{\prime \prime} b_{0}^{\prime}-a_{0}^{\prime} b_{0}^{\prime \prime}\right)=\frac{2 \pi \eta}{k^{2}} \sum_{s n m}(-)^{m} \frac{n(n+1)}{2 n+1}\left(Q^{\prime / s m n} P_{s,-m, n}^{\prime}-Q^{\prime s m n} P_{s,-m, n}^{\prime \prime}\right) .
$$

Now, following Kerns [28], we set the exciting-mode amplitudes to

$$
\begin{array}{ll}
a_{0}^{\prime \prime}=0, & P_{s m n}^{\prime \prime}=\delta_{s \sigma} \delta_{m \mu} \delta_{n v}, \\
a_{0}^{\prime}=1, & P_{s m n}^{\prime}=0 .
\end{array}
$$

${ }^{1}$ The results presented in Chapter III were previously presented in May of 1977, by the author, in lectures at the University of Colorado and at the National Bureau of Standards.

${ }^{2}$ There should be no confusion between the use of primes, in this section only, to differentiate between different fields and the use of primes elsewhere to differentiate between different coordinate systems. 
Inserting these exciting-mode amplitudes into eqs (3.2), (3.3), and (3.7) results in

$$
R_{\sigma \mu \nu}=2 \pi \frac{\eta}{\hat{\eta}_{0} k^{2}}(-)^{\mu} \frac{\nu(\nu+1)}{2 \nu+1} T^{\sigma,-\mu, \nu} .
$$

Thus, eq (3.8) relates the transmitting and receiving mode coefficients for a reciprocal antenna. Similarly, we obtain from eqs (3.3) and (3.7),

$$
S_{s,-m, n}^{\sigma,-\mu, \nu}=S_{\sigma \mu \nu}^{s m n} .
$$

Similar relationships have been obtained by Yaghjian [60] between cylindrical-wave sourcescattering matrix elements.

\subsection{Power Gain and Effective Area of an Antenna}

The most common characteristics associated with an antenna are the power gain function for a transmitting antenna and the effective-area function for a receiving antenna. These quantities are functions of direction and are strictly far-field quantities, which is appropriate for conventional antenna theory. In this section we shall express these quantities in terms of the antenna's spherical-wave source-scattering matrix parameters. The definitions used here for power gain and effective receiving area are consistent with IEEE standard No. 145 (1973); we here follow Kerns' formulation [26] in detail. The definitions of the receiving and transmitting properties are formed wholly independently of each other, without any assumption of reciprocity. For cases where reciprocity is appropriate for the antenna under consideration, the relationship between power-gain and effective-area will be given.

The power-gain function of a transmitting antenna is given by

$$
G(\theta, \phi)=4 \pi \frac{P_{r a d}}{P_{0}},
$$

where $P_{\text {rad }}$ is the power radiated per unit solid angle at large distances in the direction of $(\theta, \phi)$ and $\mathrm{P}_{\mathrm{o}}$ is the net input power to the antenna. ${ }^{3}$ The expression for $\mathrm{P}_{\mathrm{o}}$ has already been given (equation 2.4). The time-average energy flux crossing a surface area $\mathrm{A}$ is given by

$$
P=\frac{1}{2} \operatorname{Re} \int_{A} \underline{E} \times \underline{H}^{*} \cdot \underline{a}_{r} d s,
$$

where the electric and magnetic field vectors are given by eq (2.46) with $\mathrm{P}_{\mathrm{smn}}$ set equal to zero. At large distances from the source, the field in the neighborhood of a given direction behaves

${ }^{3}$ There should be no confusion between the dyadic $\underline{\underline{G}}$, the transverse vector wave function $\underline{G}$, and the power gain $\mathrm{G}$. 
locally as if it were a plane wave propagating in that direction. Consequently, from Stratton [50] we have for large distances the asymptotic relation,

$$
\underline{H}=\eta \underline{a}_{r} \times \underline{E} .
$$

Assuming that the area of integration in eq (3.11) is a local neighborhood about a given direction, then the integrand of eq (3.11) will be a constant. Consequently, the radiated power per unit solid angle in a given direction $(\theta, \phi)$ becomes

$$
P_{\text {rad }}=\frac{\eta}{2} \lim _{r \rightarrow \infty}|r \underline{E}(r, \theta, \phi)|^{2},
$$

where $|\mathrm{A}|^{2} \equiv \underline{\mathrm{A}} \cdot \underline{\mathrm{A}}^{*}$ for any vector $\underline{\mathrm{A}}$. Using eq (2.33), we obtain

$$
P_{\text {rad }}=\frac{\eta}{2}\left|\frac{1}{k} \sum_{s n m} i^{s-n-2} Q^{s m n} \underline{G}_{n}^{s m}(\theta, \phi)\right|^{2} .
$$

Consequently, from eqs (3.2) and (3.3) we obtain the power-gain function

$$
G(\theta, \phi)=\frac{4 \pi \eta}{k^{2} \hat{\eta}_{0}\left(1-|\Gamma|^{2}\right)}\left|\sum_{s n m} i^{s-n} T^{s m n} \underline{G}_{n}^{s m}(\theta, \phi)\right|^{2} .
$$

Another quantity related to the gain is the directivity, which is defined as

where $\mathrm{P}_{\text {total }}$ is the total radiated power, defined as

$$
D(\theta, \phi)=4 \pi \frac{P_{\text {rad }}}{P_{\text {toral }}},
$$

$$
P_{\text {total }}=\int_{0}^{2 \pi} d \phi \int_{0}^{\pi} \sin \theta d \theta P_{\text {rad }}(\theta, \phi) .
$$

As a consequence of eq (2.39), we obtain for the directivity the expression,

$$
D(\theta, \phi)=\left|\sum_{s n m} i^{s-n} T^{s m n} \underline{G}_{n}^{s m}(\theta, \phi)\right|^{2} /\left(\sum_{s n m} \frac{n(n+1)}{2 n+1}\left|T^{s m n}\right|^{2}\right) .
$$

The antenna efficiency is obtained by taking the ratio of the gain eq (3.15) to the directivity eq (3.18).

For an antenna operating in a receiving rather than a transmitting capacity, the counterpart of the power gain is the effective area or effective receiving cross section. Like the power gain, this quantity is a scalar function of direction and involves a far-field concept-in this case that of an incident plane wave. The effective area is defined in accordance with Kerns [26], as

$$
\sigma(\theta, \phi)=\frac{P_{A, \max }}{S_{p o y}},
$$


whcre $\mathrm{S}_{\text {poy }}$ is the magnitude of the Poynting vector for an incident plane wave arriving at the antenna from a given direction and providing a polarization match to the antenna receiving characteristic for that direction, while $\mathrm{P}_{\mathrm{A} \text {,max }}$ is the maximum available power at the antenna terminal due to this incident plane wave. Now we can write (3.2) as

$$
b_{0}=\Gamma a_{0}+b_{G},
$$

where $b_{G}$ is the generated wave produced by an equivalent waveguide source replacing the antenna. Then, if the antenna is terminated in a load or detector having a reflection coefficient $\Gamma_{\mathrm{L}}=\left(\mathrm{a}_{0}\right) /\left(\mathrm{b}_{0}\right)$, the power delivered to that load by the antenna is given by [27]

$$
P_{\text {del }}=\frac{\hat{\eta}_{0}}{2} \frac{1-\left|\Gamma_{L}\right|^{2}}{\left|1-\Gamma_{L} \Gamma\right|^{2}}\left|b_{G}\right|^{2}
$$

where we have used eq (2.4). The available power is obtained from the delivered power under the condition that the load impedance is conjugate matched to the equivalent generator impcdance, or $\Gamma_{\mathrm{L}}=\Gamma^{*}$. This results in

$$
P_{A}=\frac{\hat{\eta}_{0}}{2} \frac{\left|b_{G}\right|^{2}}{1-|\Gamma|^{2}}
$$

An cxpression for $b_{\mathrm{G}}$ is readily obtained from eq (3.2) as

$$
b_{G}=\sum_{s n m} R_{s n m} P_{s m n},
$$

where the $\mathrm{P}_{\text {smin }}$ arc the modal coefficients of the incident plane wave. There coefficients have becn obtained in Section 2.3 (and in Appendix A) as

$$
P_{s m n}=\mathscr{E}_{0} \frac{2 n+1}{n(n+1)} i^{s-n-3} \underline{G}_{n}^{s m *}(\theta, \phi) \cdot \vec{q},
$$

where $\theta, \phi$ denote the direction from which the incident plane wave is arriving, and $\vec{q}$ specifies its polarization according to eq (2.37). Now the electric field of the incident plane wave is given by

$$
\underline{E}=\mathscr{E}_{0} \underline{\hat{e}} e^{-i \underline{k} \cdot \underline{r}},
$$

where $\underline{\mathrm{k}}=\mathrm{k} \underline{\hat{k}}$. Both $\underline{\hat{\mathrm{k}}}$ and $\underline{\hat{\mathrm{e}}}$ are unit vectors related to the unit vectors $\underline{\mathrm{a}}_{\mathrm{r}}, \underline{\mathrm{a}}_{\theta}$, $\underline{\mathrm{a}}_{\phi}$ of the spherical-coordinate system by the direction of arrival $(\theta, \phi)$ of the incident plane wave and the polarization angle $\gamma$ (see Appcndix A). The magnitude of the Poynting vector is readily obtained from eq (3.25) as [50, page 284],

$$
S_{p o y}=\frac{\eta}{2}|\underline{E}|^{2}=\frac{1}{2} \eta\left|\mathscr{E}_{0}\right|^{2} .
$$


Consequently, eqs (3.22) through (3.24) and eq (3.26) yield the following expression for the available power at the antenna terminal due to an incident plane wave,

$$
P_{A}=\frac{\hat{\eta}_{0} S_{p o y}}{\eta\left(1-|\Gamma|^{2}\right)}\left|\left(\sum_{s n m} i^{s-n} \frac{2 n+1}{n(n+1)} R_{s m n} \underline{G}_{n}^{s m *}(\theta, \phi)\right) \cdot \vec{q}\right|^{2} .
$$

When the incident wave is polarization matched to the receiving antenna, we have

$$
\begin{aligned}
& \left|\vec{q} \cdot\left(\sum_{s n m} i^{s-n} \frac{2 n+1}{n(n+1)} R_{s m n} \underline{G}_{n}^{s m *}(\theta, \phi)\right)\right|^{2} \\
& =|\vec{q}|^{2}\left|\sum_{s n m} i^{s-n} \frac{2 n+1}{n(n+1)} R_{s m n} G_{n}^{s n *}(\theta, \phi)\right|^{2} .
\end{aligned}
$$

Thus, eq (3.28) shows that $\mathrm{P}_{\mathrm{A}}$ becomes $\mathrm{P}_{\mathrm{A}, \max }$ when the incident wave and the antenna are polarization matched. Now from eq (2.37) we have $|\vec{q}|=1$. Therefore, the effective receiving cross section eq (3.19) becomes,

$$
\sigma(\theta, \phi)=\frac{\hat{\eta}_{0}}{\eta\left(1-|\Gamma|^{2}\right)}\left|\sum_{s n m} i^{s-n} \frac{2 n+1}{n(n+1)} R_{s m n} \underline{G}_{n}^{s m *}(\theta, \phi)\right|^{2} .
$$

If we reverse the order of summation of the $m$ index and make use of the complex-conjugate relationship eq (2.30), then we can rewrite eq (3.29) as

$$
\sigma(\theta, \phi)=\frac{\hat{\eta}_{0}}{\eta\left(1-|\Gamma|^{2}\right)}\left|\sum_{s n m} i^{s-n} \frac{2 n+1}{n(n+1)}(-)^{m} R_{s,-m, n} \underline{G}_{n}^{s m}(\theta, \phi)\right|^{2} .
$$

Equation (3.15) expressing a transmitting antenna's power gain and eq (3.30) expressing a receiving antenna's effective receiving area were obtained without making any assumption regarding reciprocity. Consequently these expressions are equally valid for nonreciprocal as well as reciprocal antennas, for instance antennas constructed using ferrite phase shifters. However, in the event that the antenna is reciprocal, it is interesting to apply the reciprocity relation eq (3.8) to eq (3.30) to see what results. Accordingly, for the case of a reciprocal antenna the effective area expression becomes

$$
\sigma(\theta, \phi)=\frac{\eta}{\hat{\eta}_{0}\left(1-|\Gamma|^{2}\right)} \frac{4 \pi^{2}}{k^{4}}\left|\sum_{s n m} i^{s-n} T^{s m n} \underline{G}_{n}^{s m}(\theta, \phi)\right|^{2} .
$$

Upon substituting from eq (3.15) for the quantity inside the absolute-value bars in eq (3.31) we obtain

$$
\sigma(\theta, \phi)=\frac{\pi}{k^{2}} G(\theta, \phi)
$$


Equation (3.32) is the classical expression relating effective receiving cross section and power gain for a reciprocal antenna. If we write $k=\frac{2 \pi}{\lambda}$, where $\lambda$ is the wavelength in the medium exterior to the antenna, eq (3.32) assumes the more conventional form

$$
\sigma(\theta, \phi)=\frac{\lambda^{2}}{4 \pi} G(\theta, \phi) .
$$

Although it would be straightforward to write expressions for the "polarization mismatch" factor from eqs (3.27) and (3.28), corresponding to Kerns' treatment [26] of the plane-wave case, there just results ratios of complicated summations which do not admit of any ready simplification. However, it is interesting to note that the statements by Kerns in his final paragraph of Scction $1.6[25$, p. 26] regarding circular polarization match are borne out by cqs (2.42) and (3.24). Note that right- and left-hand circular polarization are characterized by $\mathrm{m}= \pm 1$, with a $180^{\circ}$ flip in the propagation direction requiring a sign switch in $\mathrm{m}$, or a complex conjugation, to preserve the handedness of the polarization. Thus, this reaffirms Kerns' statement, "that if the radiation from a reciprocal antenna in a certain direction is circularly polarized, the wave best reccived from that direction is circularly polarized in the same screw sense."

\subsection{Conclusions}

In this chaptcr wc introduced a spherical-wave source-scattering matrix representation of an antenna relating the antenna's emergent and incident waveguide-feed amplitudes to exciting and emergent modal-expansion coefficients of the electromagnetic field on the surface of a hypothctical sphere enclosing the antenna (eq (3.2) and (3.3)). We then obtained reciprocity relations between the source-scattering matrix elements for a reciprocal antenna (eq (3.8) and (3.9)). Next, we obtained expressions for power gain (3.15) and directivity (3.18) of a transmitting antenna in terms of the transmitting source-scattering matrix elements, while for a receiving antenna wc obtained an expression for the effective receiving cross section (3.30) in terms of the receiving source-scattering matrix elements. In the event that the antenna is reciprocal, we obtained the classical result (3.33) that the receiving cross section is equal to $\lambda^{2} / 4 \pi$ times the power gain.

The further usc of the source-scattering matrix elements to describe the interaction of two scparatcd antennas will be presented in Chapter V. In the next chapter we develop some functional transformations which are necessary to continue the development of our sourcescattcring matrix theory. 


\section{CHAPTER IV}

\section{FUNCTIONAL TRANSFORMATIONS INDUCED BY COORDINATE-SYSTEM ROTATIONS AND TRANSLATIONS}

\subsection{Introduction}

In this chapter we briefly state the transformation formulas needed to express the basis functions in one coordinate system as a linear combination of the basis functions in another coordinate system, where the two coordinate systems are related by coordinate transformations: specifically, a rotation or translation of coordinates. Subsequently, these analytical expressions will be used to transform thc modal-field expansion of a radiating antcnna from that antenna's coordinate system to the coordinate system of a receiving antenna. Thus, in figure 4-1 we show schematically two coupled antennas, physically separated by a distance $d$. The antenna on the left is described in terms of the unprimed ( $x-y-z)$ coordinate system, while the antenna on the right is described in terms of the doubly-primed ( $\left.x^{\prime \prime}-y^{\prime \prime}-z^{\prime \prime}\right)$ coordinate system. A rotation of coordinates carries the unprimed coordinate system into the singly-primed system, whose coordinate axes are all parallel to the axes of the doubly-primed coordinate system. Then a rigid translation of coordinates along the $z^{\prime}$-axis carries the singly-primed coordinate system into the doubly-primed coordinate system. The notation used in figure 4-l to distinguish the different coordinate system will be continued beyond the present chapter.

\subsection{The Rotation of Coordinates Transformation}

The rotation of coordinates transformation, which carries the unprimed coordinate system into the singly-primed coordinate system, can be defined in terms of three angles, $\alpha, \beta, \gamma$, known as the Eulerian angles. These three angles are pictured in figure 4-1 and also more explicitly in figure 4-2. The transformation from the unprimed coordinate system to the singly-primed coordinate system consists $[17,44]$ of three steps, as depicted in figure 4-2. All rotations are considcred positive if thcy would carry a right-handed screw in a positive direction along the rotation axis. The successivc rotation steps are:

i. A rotation about the $\mathrm{z}$-axis of magnitude $\alpha$, bringing coordinate axes $\mathrm{x}$ and $\mathrm{y}$ to the new axes $x_{1}$ and $y_{1}$.

ii. A rotation about the ncw $\mathrm{y}_{1}$-axis of magnitude $\beta$, bringing coordinate axes $\mathrm{x}_{1}$ and $\mathrm{z}$ to the new axes $x_{2}$ and $z^{\prime}$. 


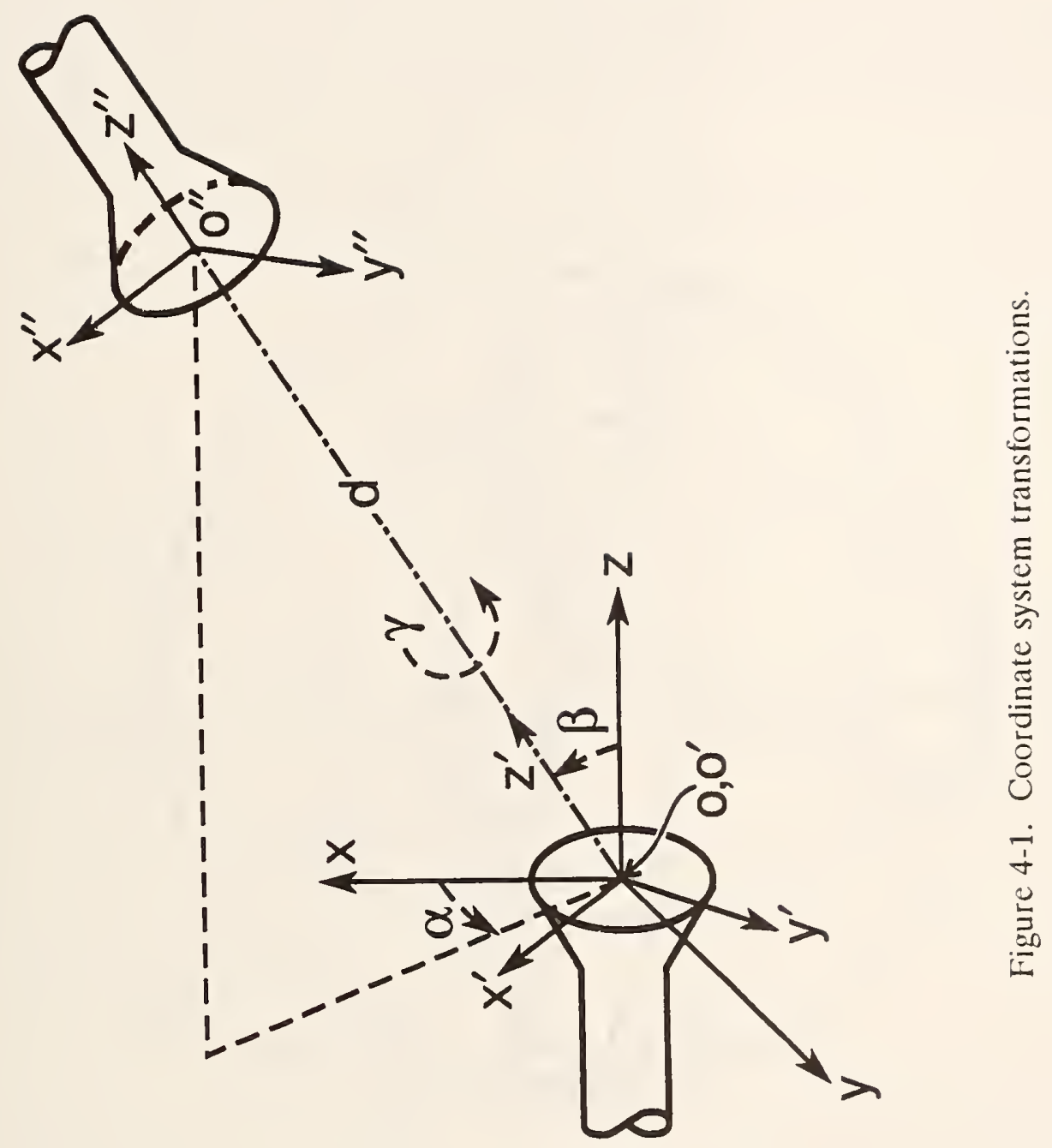




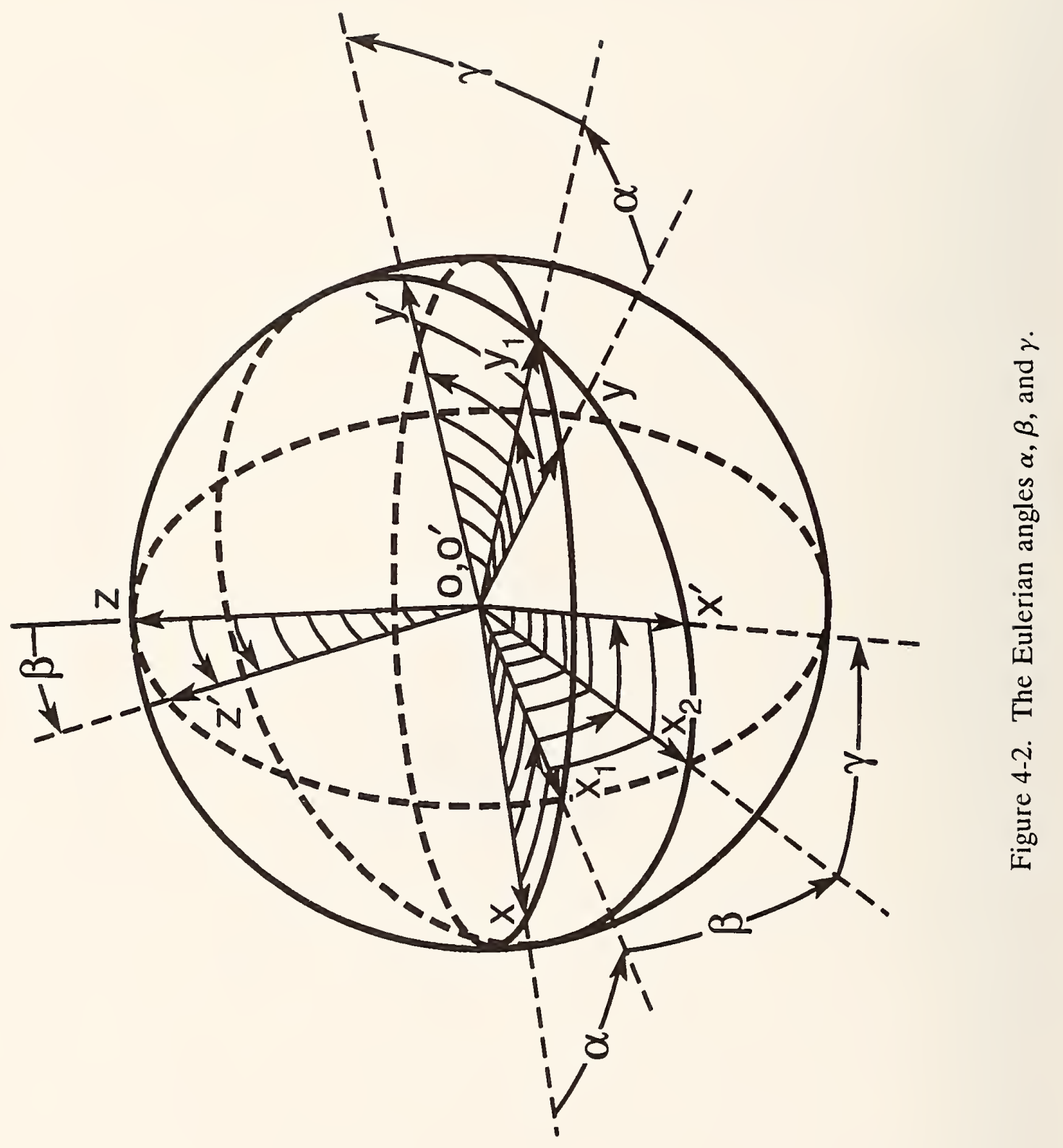


iii. A rotation about the new $z^{\prime}$-axis of magnitude $\gamma$, bringing axes $x_{2}$ and $y_{1}$ to the new axes $\mathrm{x}^{\prime}$ and $\mathrm{y}^{\prime}$.

Let $\theta^{\prime}, \phi^{\prime}$ denote the spherical-coordinate angles of a point with respect to the singly primed coordinate system, and similarly let $\theta, \phi$ denote the spherical-coordinate angles of the same point but with respeet to the unprimed eoordinate system. The set of spherieal harmonies $\mathrm{Y}_{\mathrm{nm}}(\theta, \phi)$ of degree $\mathrm{n}$ and order $\mathrm{m}$ form a complete set of basis functions on the surface of a sphere [50]. These basis funetions transform from the unprimed eoordinate system to the singly primed coordinate system as [44]

$$
Y_{n m}\left(\theta^{\prime}, \phi^{\prime}\right)=\sum_{\mu=-n}^{n} Y_{n \mu}(\theta, \phi) D_{\mu m}^{n}(\alpha, \beta, \gamma)
$$

where ${ }^{1}$

$$
D_{\mu m}^{n}(\alpha, \beta, \gamma)=e^{-i \mu \alpha} d_{\mu m}^{n}(\beta) e^{-i m \gamma}
$$

A elosed form expression for the $d_{\mu m}^{n}(\beta)$ funetion is given [44] by

$$
d_{\mu m}^{n}(\beta)=\sqrt{\frac{(n+\mu) !(n-\mu) !}{(n+m) !(n-m) !}} \sum_{\sigma}(-)^{\sigma}\left(\begin{array}{c}
n-m \\
n-\mu-\sigma
\end{array}\right)\left(\begin{array}{c}
n+m \\
\sigma
\end{array}\right)\left(\cos \frac{\beta}{2}\right)^{2 n+m-\mu-2 \sigma}\left(-\sin \frac{\beta}{2}\right)^{\mu-m+2 \sigma} .
$$

The sum is over all positive integers for which the terms in the series do not vanish [58]. Now then, it is readily shown (see, for instance, [12]) using eq (2.10) and Rose's derivation of Rogdriguez's formula [44, Appendix III] that

$$
d_{m o}^{n}(\beta)=\sqrt{\frac{(n-m) !}{(n+m) !}} P_{n}^{m}(\cos \beta)
$$

Moreover, by letting $\beta$ and $\gamma$ assume the special values $\beta=\theta^{\prime}, \gamma=\pi-\phi^{\prime}$ we obtain, using eq (4.2), the special ease result [58, appendix A.3]

$$
Y_{n m}\left(\theta^{\prime}, \phi^{\prime}\right)=(\text { const. }) d_{m o}^{n}\left(\theta^{\prime}\right) e^{i m \phi^{\prime}},
$$

where the "const." in eq (4.4) is independent of $\theta^{\prime}, \phi^{\prime}$, or m. Thus, upon substituting eq (4.3) into (4.4) and comparing that result with eq (2.8), it becomes obvious how the normalization constant in eqs (2.8) and (2.14) was chosen.

${ }^{1}$ Here and throughout this work, we adopt the definitions in Rose [44] for the rotation of coordinates transformation matrix $D$. Note that Wigner [58], for instance, uses the slightly different but consistent definition $\tilde{D}_{\mu m}^{n}(\alpha, \beta, \gamma)=D_{-\mu,-m}^{n}(\alpha, \beta, \gamma)$, where his transformation corresponding to (4.1) represents a rotation of field points about stationary coordinate axes, whereas we adopt the convention that the field points remain stationary while the eoordinate axes rotate. 
We now consider the situation where the basis functions are used for the series expansion of some scalar function of the angular coordinates

$$
\epsilon(\theta, \phi)=\sum_{j, m} Y_{j m}(\theta, \phi) q_{j m}
$$

where a shorthand notation is introduced for the double sum,

$$
\sum_{j, m} \equiv \sum_{j=1}^{\infty} \sum_{m=-j}^{j}
$$

In (4.5) the $\mathrm{q}_{\mathrm{jm}}$ are the series-expansion coefficients, and $\epsilon(\theta, \phi)$ is presumed to be an arbitrary scalar function of the angular coordinates $\theta$ and $\phi$. Now consider a rotated coordinate system with coordinates $\theta^{\prime}, \phi^{\prime}$ for the same function. That is, the function $\epsilon^{\prime}\left(\theta^{\prime}, \phi^{\prime}\right)$ is the same function in terms of position in space as $\epsilon(\theta, \phi)$, so that $\epsilon^{\prime}\left(\theta^{\prime}, \phi^{\prime}\right)=\epsilon(\theta, \phi)$. Now a seriesexpansion representation for $\epsilon^{\prime}$ can be written in terms of the basis functions, resulting in

$$
\epsilon^{\prime}\left(\theta^{\prime}, \phi^{\prime}\right)=\sum_{j, \mu} q_{j \mu}^{\prime} Y_{j \mu}\left(\theta^{\prime}, \phi^{\prime}\right)
$$

where the $q_{j \mu}^{\prime}$ are the expansion coefficients of the new function $\epsilon^{\prime}$. These new series- expansion coefficients are related to the original $\mathrm{q}_{\mathrm{jm}}$ expansion coefficients by [18]

$$
q_{j \mu}^{\prime}=\sum_{m=-j}^{j} q_{j m} D_{\mu m}^{j}(-\gamma,-\beta,-\alpha)
$$

where $-\gamma,-\beta,-\alpha$ is the inverse rotation to the rotation $\alpha, \beta, \gamma$. Equation (4.7) follows directly from the group property [58]

$$
\sum_{\mu=-j}^{j} D_{m^{\prime} \mu}^{j}(\alpha, \beta, \gamma) D_{\mu m}^{j}(-\gamma,-\beta,-\alpha)=D_{m^{\prime} m}^{j}(0,0,0)=\left\{\begin{array}{l}
1, m^{\prime}=m \\
0, m^{\prime} \neq m
\end{array} .\right.
$$

Moreover, since the rotation transformation is a unitary transformation, we have [18]

$$
D_{\mu m}^{j}(-\gamma,-\beta,-\alpha)=D_{m \mu}^{j *}(\alpha, \beta, \gamma) \text {, }
$$

where the asterisk again denotes the complex conjugate.

A recursion relation between the $\mathrm{d}_{\mathrm{m} \mathbf{m}^{\prime}}^{\mathrm{j}}(\beta)$ functions has been obtained by Fano and Racah [18]. Upon restructuring their formula to account for the different definitions of the $\mathrm{d}_{\mathrm{m}}^{\mathrm{n} m}(\beta)$ functions between Rose and Wigner, we have the recursion relations

$$
\begin{aligned}
& \pm \sqrt{\ell(\ell+1)-m^{\prime}\left(m^{\prime}-1\right)}(1 \pm \cos \beta) d_{m^{\prime}-1, m}^{\ell}(\beta)-m^{\prime} \sin \beta d_{m^{\prime} m}^{\ell}(\beta) \\
= & \sqrt{\ell(\ell+1)-m(m \pm 1)}(1 \pm \cos \beta) d_{m^{\prime}, m \pm 1}^{\ell}(\beta) \pm m \sin \beta d_{m^{\prime} m}^{\ell}(\beta) .
\end{aligned}
$$


The two relations (one with the leading plus sign, one with the leading minus sign) shown in eq (4.10) will be used to obtain an important numerical computation algorithm for computing the $\mathrm{d}_{\mathrm{m}^{\prime} \mathrm{n}}^{\mathrm{j}}(\beta)$ functions.

Using a similarity transformation, Edmonds [17] obtained the relationship

$$
d_{m^{\prime} m}^{\ell}(\beta)=e^{i\left(m^{\prime}-m\right) \frac{\pi}{2}} \sum_{p=-\ell}^{\ell} \Delta_{p m^{\prime}}^{\ell} \Delta_{p m}^{\ell} e^{-i p \beta},
$$

where

$$
\Delta_{m^{\prime} m}^{\ell} \equiv d_{m^{\prime} m}^{\ell}\left(\frac{\pi}{2}\right) \text {. }
$$

Equation (4.11) is valid for both Wigner's definition and for Rose's definition of the $\mathrm{d}_{\mathrm{m}^{\prime} \mathrm{m}}^{\ell}(\beta)$ functions, as long as one is consistent in defining $\Delta_{\mathrm{m}^{\prime} \mathrm{m}}^{\ell}$ in the same system. Equation (4.11) provides the basis for efficient numerical computations, as discussed in the next scction.

\subsection{Application of the Fano-Racah Recursion Formula}

\subsubsection{The Derivation of a Numerical Calculation Formula}

The result, eq (4.11), is very convenient for evaluating the d's numerically, since fast Fourier transform (FFT) algorithms are readily available. The problem is to find efficient techniques for evaluating the deltas. The most efficient way of computing such quantities is a recursion relationship. However, a recursion relationship which is numerically stable must be used to obtain success.

A potential candidate for a recursion relationship among the deltas can be obtained from eq (4.10). These two cxpressions, one with a leading plus sign and one with a leading minus sign, reduce to the following upon specifying $\beta=\pi / 2$,

$$
\pm \sqrt{\ell(\ell+1)-m^{\prime}\left(m^{\prime}-1\right)} \Delta_{m^{\prime}-1, m}^{\ell}=\left(m^{\prime} \pm m\right) \Delta_{m^{\prime} m}^{\ell}+\sqrt{\ell(\ell+1)-m(m \pm 1)} \Delta_{m^{\prime}, m \pm 1}^{\ell} .
$$

Unfortunately, neither of these formulas proved to be stable enough for numerical recursion. However, in the case of a recurrence relation of the form $y_{n+1}+a_{n} y_{n}+b_{n} y_{n-1}=0$, $\mathrm{n}=1,2,3, \ldots$, a well formulated answer exists for resolving numerical stability problems. Two linearly independent solutions of this three-term recurrence relation exist; one of these can be computed by forward recursion and one by backward recursion [20]. Thus, one is (normally) guaranteed numerically stable "dominant" and "minimal" solutions. In the case of the Bessel function recursion relation, for instance, both forward and backward recursions are self convergent to these two types of solutions [34], in the sense that rounding and initial value errors 
correct themselves through the recursive process, as indeed is predicted by Gautschi's theory [20]. This situation holds for any three-term recurrence relation for which the solutions have a magnitude gradient for increasing or decreasing indexes n. However, if no magnitude gradient exists, then self correction of rounding and initial-value errors may not exist, and extreme care must be exercised in the selection of the recursion algorithm. Finally, if the ratio of the minimal to the dominant solution does not asymptotically decrease, the theory [20] breaks down. Such a case, fortunately, should be rare.

In the present instance, a strong magnitude gradient over the $\mathrm{m}, \mathrm{m}^{\prime}$ plane does exist, so that all that is required is to convert eq (4.13) into a three-term recursion relation having effectively a single index constrained to a line of integers in the $\mathrm{m}, \mathrm{m}^{\prime}$ plane. In order to obtain such an expression, we can use the relation [17]

$$
\Delta_{m^{\prime} m}^{\ell}=(-)^{\ell-m} \Delta_{-m^{\prime}, m}^{\ell}
$$

to modify eq (4.13). Then, upon adding the original expression (4.13) to the modified expression, we obtain ${ }^{2}$

$$
\sqrt{\ell(\ell+1)-m^{\prime}\left(m^{\prime}+1\right)} \Delta_{m^{\prime}+1, m}^{\ell}+\sqrt{\ell(\ell+1)-m^{\prime}\left(m^{\prime}-1\right)} \Delta_{m^{\prime}-1, m}^{\ell}=2 m \Delta_{m^{\prime} m}^{\ell} \text {. }
$$

This recursion formula satisfies all the conditions for stability. The gradient across the $\mathrm{m}, \mathrm{m}^{\prime}$ plane requires backwards recursion. Starting with $\mathrm{m}^{\prime}=\ell$, the recursion algorithm, eq (4.15), can be uscd to compute dcltas having successively smaller $\mathrm{m}^{\prime}$ indices until $\mathrm{m}^{\prime}=0$ is reached. Since eq (4.14) can be used to obtain values of $\Delta$ having negative indices $\mathrm{m}^{\prime}$, the recursive process can stop at $\mathrm{m}^{\prime}=0$. Also, we have the result [17],

$$
\Delta_{m^{\prime} m}^{\ell}=(-)^{\ell-m^{\prime}} \Delta_{m^{\prime},-m}^{\ell}
$$

so that the recursive process can be restricted to $\mathrm{m}$ and $\mathrm{m}$ ' both positive.

The initial starting values for the recursion relation eq (4.15) can be obtained from the serics expansion for $\mathrm{d}_{\mathrm{m}^{\prime} \mathrm{m}}^{\ell}(\beta)$. Thus, for $\mathrm{m}^{\prime}=\ell$ that expression becomes

$$
d_{\ell m}^{\ell}(\beta)=\sqrt{\frac{(2 \ell) !}{(\ell+m) !(\ell-m) !}}\left(\cos \frac{\beta}{2}\right)^{\ell+m}\left(-\sin \frac{\beta}{2}\right)^{\ell-m} .
$$

A similar result has also been given by Edmonds [17]. Upon substituting $\beta=\pi / 2$ into the preceding expression we obtain

${ }^{2}$ This formula for computing the deltas was originally presented in class note handouts to participants at the August 1977 Near-Field Antenna Measurements course held at the National Bureau of Standards, U.S. Dept. of Commcree, Boulder, Colorado. 


$$
\Delta_{\ell m}^{\ell}=\frac{(-)^{\ell-m}}{2^{\ell}} \sqrt{\frac{(2 \ell) !}{(\ell+m) !(\ell-m) !}} .
$$

The other starting value is $\Delta_{\ell+1,01}^{\ell}=0$. The effectiveness of the recursive process can be checked by comparing the result obtained using eq (4.15) at $\mathrm{m}^{\prime}=0$ with a closed-form expression for $\Delta_{\text {oni }}^{\ell}$. Thus, from eq (4.3) we obtain

$$
\Delta_{\text {om }}^{\ell}=(-)^{m} \sqrt{\frac{(\ell-m) !}{(\ell+m) !}} P_{\ell}^{m}(0) .
$$

Using the formula [1] for the value of the associated Legendre function for zero argument,

$$
P_{v}^{\mu}(0)=2^{\mu} \pi^{-1 / 2} \cos \left(\frac{\nu+\mu}{2} \pi\right) \Gamma\left(\frac{1}{2} \nu+\frac{1}{2} \mu+\frac{1}{2}\right) / \Gamma\left(\frac{1}{2} \nu-\frac{1}{2} \mu+1\right),
$$

we obtain the result

$$
\Delta_{o m}^{\ell}= \begin{cases}\frac{(-)^{\frac{\ell-m}{2}}}{2^{\ell}} \frac{\sqrt{(\ell+m) !(\ell-m) !}}{\left(\frac{\ell+m}{2}\right) !\left(\frac{\ell-m}{2}\right) !}, & \ell-m \text { even } \\ 0 \quad, \quad l-m \text { odd } .\end{cases}
$$

Computations using eq (4.15) were carried out and the results checked by evaluating eq (4.18). Computational results were accurate within a relative error of $10^{-10}$ (the available precision of the computer) for all $\ell$ and $m$ such that $0 \leq \mathrm{m} \leq \ell \leq 120$.

\subsubsection{Analytical Formula Relating Rotation Coefficients to Associated Legendre Functions}

In addition to the numerical computation problem, some interesting theoretical applications result from the Fano-Racah recursion relations. For instance, the series expansion for the $\mathrm{d}_{\mu \mathrm{m}}^{\mathrm{n}}(\beta)$ coefficients, which follows eq (4.2), can be derived using these recursion relations [18]. Here, we develop contiguity relationships between adjacent columns of the representation coefficient matrix. This in turn will lead to establishing an equality between the expression for the radiated field and the expression for the signal received by a dipole antenna. Expressions for the radiated field have already been given in Chapter II, while expressions for the coupling between two antennas will be developed in Chapter V.

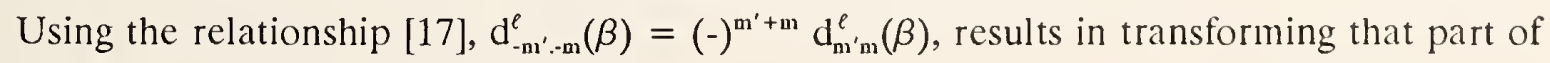
eq (4.10) with the leading minus sign into 


$$
\begin{aligned}
& \sqrt{\ell(\ell+1)-m^{\prime}\left(m^{\prime}+1\right)}(1-\cos \theta) d_{m^{\prime}+1, m}^{\ell}(\theta) \\
& =\left(m-m^{\prime}\right) \sin \theta d_{m^{\prime} m}^{\ell}(\theta)-\sqrt{\ell(\ell+1)-m(m+1)}(1-\cos \theta) d_{m^{\prime}, m+1}^{\ell}(\theta) .
\end{aligned}
$$

Combining this result with the expression (4.10) having the leading plus sign produces

$$
\begin{aligned}
d_{m^{\prime}, m+1}^{\ell}(\theta)= & \frac{1+\frac{m}{m^{\prime}}}{2} \frac{1-\cos \theta}{\cos \theta-\frac{m}{m^{\prime}}} \sqrt{\frac{\ell(\ell+1)-m^{\prime}\left(m^{\prime}+1\right)}{\ell(\ell+1)-m(m+1)}} d_{m^{\prime}+1, m}^{\ell}(\theta) \\
& +\frac{1-\frac{m}{m^{\prime}}}{2} \frac{1+\cos \theta}{\cos \theta-\frac{m}{m^{\prime}}} \sqrt{\frac{\ell(\ell+1)-m^{\prime}\left(m^{\prime}-1\right)}{\ell(\ell+1)-m(m+1)}} d_{m^{\prime}-1, m}^{\ell}(\theta) .
\end{aligned}
$$

Similarly, using [12] $\mathrm{d}_{\mathrm{m}^{\prime} \mathrm{m}}^{\ell}(\pi-\beta)=(-)^{\ell-\mathrm{m}^{\prime}} \mathrm{d}_{\mathrm{m}^{\prime},-\mathrm{m}}^{\ell}(\beta)$ in that part of expression (4.10) with the leading minus sign and then recombining with that part having the leading plus sign results in

$$
\begin{aligned}
d_{m^{\prime}, m-1}^{\ell}(\theta) & =\frac{1-\frac{m}{m^{\prime}}}{2} \frac{1+\cos \theta}{\cos \theta-\frac{m}{m^{\prime}}} \sqrt{\frac{\ell(\ell+1)-m^{\prime}\left(m^{\prime}+1\right)}{\ell(\ell+1)-m(m-1)}} d_{m^{\prime}+1, m}^{\ell}(\theta) \\
+ & \frac{1+\frac{m}{m^{\prime}}}{2} \frac{1-\cos \theta}{\cos \theta-\frac{m}{m^{\prime}}} \sqrt{\frac{\ell(\ell+1)-m^{\prime}\left(m^{\prime}-1\right)}{\ell(\ell+1)-m(m-1)}} d_{m^{\prime}-1, m}^{\ell}(\theta) .
\end{aligned}
$$

Our major interest in expressions (4.19) will be for the case $m=0$. We also require, in view of eq (4.3), some analytical relationships between contiguous Legendre functions. Thus, we have [3]

$$
\begin{gathered}
P_{v}^{\mu+1}(x)+(v-\mu+1)(v+\mu) P_{v}^{\mu-1}(x)=-2 \mu x\left(1-x^{2}\right)^{-1 / 2} P_{v}^{\mu}(x) \\
P_{v}^{\mu+1}(x)-(v-\mu+1)(v+\mu) P_{v}^{\mu-1}(x)=2\left(1-x^{2}\right)^{-1 / 2}\left[v x P_{v}^{\mu}(x)-(v+\mu) P_{v-1}^{\mu}(x)\right] \\
=-2\left(1-x^{2}\right)^{1 / 2} \frac{d P_{v}^{\mu}(x)}{d x} .
\end{gathered}
$$

Upon substituting $\mathrm{x}=\cos \theta$ into eq (4.20) and noting that

$$
-\left.\left(1-x^{2}\right)^{1 / 2} \frac{d P_{v}^{\mu}(x)}{d x}\right|_{x=\cos \theta}=\frac{\partial P_{v}^{\mu}(\cos \theta)}{\partial \theta},
$$


the two eqs (4.20) become, using eq (4.3)

$$
\begin{gathered}
\sqrt{(n-m)(n+m+1)} d_{m+1,0}^{n}(\theta)+\sqrt{(n-m+1)(n+m)} d_{m-1,0}^{n}(\theta) \\
=\frac{-2 m \cos \theta}{\sin \theta} d_{m o}^{n}(\theta), \\
\sqrt{(n-m)(n+m+1)} d_{m+1,0}^{n}(\theta)-\sqrt{(n-m+1)(n+m)} d_{m-1,0}^{n}(\theta) \\
=2 \frac{\partial d_{m o}^{n}(\theta)}{\partial \theta} .
\end{gathered}
$$

Consequently, setting $m=0$ in eqs (4.19) and using the two eqs (4.21) to simplify the results produces the relationships

$$
\begin{aligned}
& d_{m, 1}^{n}(\theta)=\frac{1}{\sqrt{n(n+1)}}\left(\frac{-m}{\sin \theta} d_{m 0}^{n}(\theta)-\frac{\partial d_{m o}^{n}(\theta)}{\partial \theta}\right) \\
& d_{m,-1}^{n}(\theta)=\frac{1}{\sqrt{n(n+1)}}\left(\frac{-m}{\sin \theta} d_{m 0}^{n}(\theta)+\frac{\partial d_{m o}^{n}(\theta)}{\partial \theta}\right) .
\end{aligned}
$$

Equation (4.3) shows that the right-hand side of eqs (4.22) can be expressed in terms of an associated Legendre function and its derivative. In Chapter VII these equations will play a significant role in uniting two distinct theoretical formulations.

\subsection{The Translation of Coordinates Transformation}

The motivation for a rigid coordinate system translation along the $z^{\prime}$-axis, between the singly primed and the doubly primed coordinate systems, is presented in Chapter $\mathrm{V}$ and is depicted in figure 5-2. The translation of the vector spherical-wave functions (see for instance, Stein [49]) from the doubly primed to the singly primed coordinate system, as shown in figure $4-1$, is given by

$$
\underline{M}_{\mu n}^{(1)^{\prime}}=\sum_{\nu=(1,|\mu|)}^{\infty}\left(A_{\mu \nu}^{\mu n} \underline{M}_{\mu \nu}^{\prime \prime}+B_{\mu \nu}^{\mu n} \underline{N}_{\mu \nu}^{\prime \prime}\right), r^{\prime \prime}<d
$$

and

$$
\underline{N}_{\mu n}^{(1)^{\prime}}=\sum_{\nu=(1,|\mu|)}^{\infty}\left(A_{\mu \nu}^{\mu n} \underline{N}_{\mu \nu}^{\prime \prime}+B_{\mu \nu}^{\mu n} \underline{M}_{\mu \nu}^{\prime \prime}\right), r^{\prime \prime}<d,
$$

where $\underline{M}_{\mu n}^{(1)^{\prime}}, \underline{N}_{\mu n}^{(1)^{\prime}}$ are vector spherical-wave functions, defined with respect to the singly primed coordinate system, which contain the spherical Hankel function $h_{n}^{(1)}\left(k r^{\prime}\right)$ as the radial-coordinate 
function (refer to eq (2.14)); while $\underline{M}_{\mu n}^{\prime \prime}, \underline{N}_{\mu n}^{\prime \prime}$ are vector spherical-wave functions, defined with respect to the doubly primed coordinate system, which contain the spherical Bessel function $\mathrm{j}_{\mathrm{a}}\left(\mathrm{kr}^{\prime \prime}\right)$ as the radial-coordinate function (refer to eq (2.8)). The notation, $\nu=(1,|\mu|)$ specifies that the summation starts at the value $v=1$ or $v=\mid \mu h$ whichever is larger. In the above,

$$
\begin{gathered}
A_{\mu \nu}^{\mu n}=(-)^{\mu} i^{v-n} \frac{v+1 / 2}{v(v+1)} \sqrt{\frac{(v+\mu) !(n-\mu) !}{(v-\mu) !(n+\mu) !}} \sum_{p} i^{p}[n(n+1)+v(v+1)-p(p+1)] \\
\times a(\mu, n,-\mu, v, p) h_{p}^{(1)}(k d)
\end{gathered}
$$

Here, the summation over $p$ sums every other term between $b-n l \leq p \leq n+v$. Also,

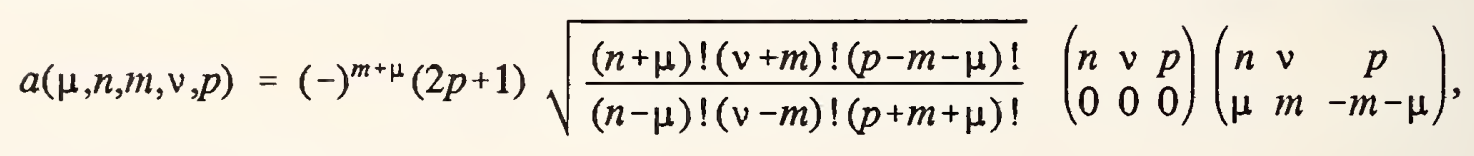

where the quantities

$$
\left(\begin{array}{lll}
j_{i} & j_{2} & j_{3} \\
m_{1} & m_{2} & m_{3}
\end{array}\right)
$$

are the so called Wigner 3-j symbols [17].

The preceding expressions for $\mathrm{A}_{\mu \nu}^{\mu \mathrm{n}}$ and $\mathrm{B}_{\mu \nu}^{\mu \mathrm{n}}$ are identical to those given by Bruning and Lo [9], except for the factor

$$
\sqrt{\frac{(v+\mu) !(n-\mu) !}{(v-\mu) !(n+\mu) !}}
$$

in eq (4.24). This additional factor arises from the square-root normalization factor in eqs (2.8) and (2.14), which in turn arises from eq (4.4). That is, the square-root term in eq (4.24) was included to make the translation of coordinates transformation compatible with the rotation of coordinates transformation.

Translation expressions for the opposite direction to that used for eq (4.23) are [9]

$$
\underline{M}_{\mu n}^{(1)^{\prime \prime}}=\sum_{v=(1,|\mu|)}^{\infty}\left(a_{\mu v}^{\mu n} \underline{M}_{\mu v}^{\prime}+b_{\mu v}^{\mu n} \underline{N}_{\mu v}^{\prime}\right), r^{\prime}<d
$$

and

$$
\underline{N}_{\mu n}^{(1)^{\prime \prime}}=\sum_{\nu=(1,|\mu|)}^{\infty}\left(a_{\mu \nu}^{\mu n} \underline{N}_{\mu v}^{\prime}+b_{\mu v}^{\mu n} \underline{M}_{\mu v}^{\prime}\right), r^{\prime}<d
$$

where

$$
a_{\mu v}^{\mu n}=(-)^{n+v} A_{\mu v}^{\mu n} ; b_{\mu v}^{\mu n}=(-)^{n+v+1} B_{\mu \nu}^{\mu n}
$$


Next, we wish to consider some special cases of eqs (4.24) for the translation coefticients, and also present a numerical recursion algorithm for computing the $\mathrm{a}(\mu, \mathrm{n},-\mu, \nu, \mathrm{p})$ coefficients. First, we note [17] that

$$
\left(\begin{array}{lll}
j_{1} & j_{2} & j_{3} \\
m_{1} & m_{2} & m_{3}
\end{array}\right)=(-)^{j_{1}+j_{2}+j_{3}}\left(\begin{array}{ccc}
j_{1} & j_{2} & j_{3} \\
-m_{1} & -m_{2} & -m_{3}
\end{array}\right) .
$$

Consequently, we see from eq (4.25) that

$$
\sqrt{\frac{(\nu+\mu) !(n-\mu) !}{(\nu-\mu) !(n+\mu) !}} a(\mu, n,-\mu, \nu, p)
$$

is invariant with respect to a change in the sign of $\mu$. It can also be shown [9], using the recursion relations between contiguous coefficients, that

$$
a(1, n,-1, v, p)=-\frac{n(n+1)+v(v+1)-p(p+1)}{2 v(v+1)} a(0, n, 0, v, p)
$$

and

$$
a(0, n, 0, v, p)=(2 p+1) \frac{(v+p-n) !(n+p-v) !(n+v-p) !}{(n+v+p+1) !}\left[\frac{\left(\frac{n+v+p}{2}\right) !}{\left(\frac{v+p-n}{2}\right) !\left(\frac{n+p-v}{2}\right) !\left(\frac{n+v-p}{2}\right) !}\right]^{2}
$$

When $\mu$ and $v$ are both equal to unity, the expressions for the A and B coefficients in eq (4.24) have particularly simple forms. Thus, from eqs (4.28) and (4.29) we obtain

$$
a(1, n,-1,1, n+1)=-a(1, n,-1,1, n-1)=\frac{n(n+1)}{2(2 n+1)} .
$$

Consequently, using the recursion relations ([1], [37]) for the spherical Hankel functions

$$
\begin{aligned}
\frac{h_{n}^{(1)}(k r)}{k r} & =\frac{1}{2 n+1}\left(h_{n-1}^{(1)}(k r)+h_{n+1}^{(1)}(k r)\right) \\
\frac{1}{k r} \frac{\partial}{\partial r}\left(r h_{n}^{(1)}(k r)\right) & =\frac{1}{2 n+1}\left((n+1) h_{n-1}^{(1)}(k r)-n h_{n+1}^{(1)}(k r)\right)
\end{aligned}
$$

we obtain the results

$$
\begin{gathered}
A_{11}^{1 n}=A_{-11}^{-1 n}=\frac{3}{2 \sqrt{2}} \sqrt{n(n+1)} \frac{1}{k d} \frac{\partial}{\partial d}\left(d h_{n}^{(1)}(k d)\right) \\
B_{11}^{1 n}=-B_{-11}^{-1 n}=i \frac{3}{2 \sqrt{2}} \sqrt{n(n+1)} h_{n}^{(1)}(k d) .
\end{gathered}
$$


Next, we need a simple recursion process that can be used to calculate the summation coefficients in eq $(4.24)$ for the case $\mu= \pm 1$. Thus, we have

$$
\begin{gathered}
a(0, n, 0,1, n+1)=\frac{n+1}{2 n+1} \\
a(0, n, 0, v, n+v)=\frac{2 v-1}{2 n+2 v-1} \times \frac{n+v}{v} a(0, n, 0, v-1, n+v-1)
\end{gathered}
$$

and

$$
a(0, n, 0, v, p-2)=\frac{(v+p-n)(n+p-v)(n+v+p+1)(n+v-p+1)}{(v+p-n-1)(n+p-v-1)(n+v+p)(n+v-p+2)} \frac{2 p-3}{2 p+1} a(0, n, 0, v, p)
$$

These results are readily obtained from eq (4.25). Equations (4.33) and (4.34) are compatible with Bruning and Lo's initial value formula for a general three-term recursion relation, while eq (4.35) is equivalent to a special case of their recursion relation. In practice, eq (4.33) serves as an initial value formula for the recursive relation eq (4.34), which in turn serves as an initial value formula for eq (4.35). Then, for each $\mathrm{n}, v$ combination, eq (4.35) serves as a recursive relation to get all the $a(0, n, 0, v, p)$ coefficients for $|n-v| \leq p<n+v$. Note that a simple test of when to stop generating coefficients is to test the recursion formula eq (4.35) for a zero numerator.

Finally, the result eq (4.35) may be combined with eq (4.28) to obtain an expression for the product of the a-coefficients with the square-root factor in eq (4.24) for the case $\mu= \pm 1$, resulting in

$$
\sqrt{\frac{(n-\mu) !(v+\mu) !}{(n+\mu) !(v-\mu) !}} a(\mu, n,-\mu, v, p)_{\mu= \pm 1}=\frac{p(p+1)-n(n+1)-v(v+1)}{2 \sqrt{n(n+1) v(v+1)}} a(0, n, 0, v, p) \text {. }
$$

With the preceding results, the only remaining item that is required for the calculation of $A_{ \pm 1, v}^{ \pm 1, n}$ and $B_{ \pm 1, v}^{ \pm 1, n}$ is the calculation of the spherical Hankel functions $h_{p}^{(1)}(k d)$. This is readily accomplished using the recursion formulas [34] for the logarithmic derivatives of the spherical Hankel functions,

$$
\chi_{n}(z) \equiv \frac{d}{d z} \ln \left[z h_{n}^{(1)}(z)\right]
$$

Thus, the following relation is numerically stable under forward recursion for the calculation of $\chi_{\mathrm{n}}(\mathrm{z})$,

$$
\chi_{n}(z)=-\frac{n}{z}+\frac{1}{\frac{n}{z}-\chi_{n-1}(z)}
$$

The initial value for this recursion relation is $\chi_{\mathrm{o}}(\mathrm{z})=\mathrm{i}$. Then, since 


$$
\frac{h_{n-1}^{(1)}(z)}{h_{n}^{(1)}(z)}=\frac{n}{z}+\chi_{n}(z)
$$

we can readily obtain the result

$$
h_{n}^{(1)}(z)=\left\{\frac{n}{z}-\chi_{n-1}(z)\right\} h_{n-1}^{(1)}(z),
$$

where the initial value for eq $(4.38)$ is

$$
h_{0}^{(1)}(z)=\frac{-i}{z} e^{i z}
$$

\subsection{Conclusions}

In this chaptcr, wc have presented the basic equations for a rotation of coordinates transformation, dcfining notations that will be used in our application of these expressions. As a part of this dcvelopment, eq (4.11) introduces the Fourier series expansion of the rotation transformation's representation coefficients. Then, numerically stable recursion relations for calculating the corresponding Fourier-series expansion coefficients are developed in eq (4.15) and (4.17). Also, expressions are developed relating elements of the representation-coefficient matrix to the associated Legendre function and its derivative (eq (4.3) and (4.22)).

Next, eq (4.23) through (4.25) present the basic equations for a translation of coordinates transformation. From these equations, we were able to relate the lowest-order vector spherical-wave function translation coefficients to the spherical Hankcl function (eq (4.31)) or its derivative (eq (4.32)). This result, along with eq (4.22) relating rotation-transformation cocfficicnts to associated Legendre functions, forms the basis for our ability to equate the expression for the emergent field in eq (2.42) to the expression, to be given in Chapter VII, for the signal received by an ideal dipole.

The chapter is concluded with the development of an efficient numerical algorithm for calculating the translation coefficients (eq (4.33) through (4.39)), which rcsult forms a part of the spherical-scanning algorithm to be given in Chapter VIII. In the next chapter we return to dcveloping the scattering theory. 


\section{CHAPTER V}

\section{SCATTERING MATRIX ANALYSIS OF COUPLED ANTENNAS: GENERAL SOLUTION FOR SYSTEM TWO-PORT}

\subsection{Introduction}

We consider a system consisting of a pair of antennas operating in a homogeneous, isotropic medium, shown schematically in figure 5-1. We are interested in this system primarily as a transmission system, with one antenna transmitting and the other receiving. The complete treatment of such a transmission system includes the effects of scattering by each antenna; consequently our analysis also treats the case where one antenna acts in both the transmitting and receiving modes, while the other antenna acts primarily as a scattering object.

Three separate coordinate systems arc depicted in figure 5-1. The doubly primed coordinate system, associated with the antenna on the right, represents the result of a rigid coordinate system translation from the singly primed coordinate system. In turn, the singly primed coordinate system represents the result of a coordinate system rotation from the unprimed coordinate system. Thus, the singly primed and the unprimed coordinate systems have a common origin, while corresponding Cartesian-coordinate axes in the singly primed and doubly primed coordinate systems are parallel. The antenna on the left in figure 5-1 is associated with the unprimed coordinate system. To emphasize this, the antenna system on the lcft in figure 5-1 is designated the unprimed antenna system, while the one on the right is designated the doubly primed antenna system. A set of incident and emergent spatial modes is associated with each antenna, along with a single mode propagating in the feed to each antenna. Thus, both antennas are separately represented as depicted in figure 2-1.

One distinction between the two antennas is that they are oriented differently with respect to their designating coordinate systems. Thus, the z-axis is shown pointing out of the antenna in the unprimed coordinate system, while the $\mathrm{z}^{\prime \prime}$-axis is shown pointing into the antcnna in the doubly primed coordinate systcm. From the standpoint of using each antenna's spherical scattcring-matrix represcntation, this distinction is irrelevant. However, it will play a part subscquently, when the scattering-matrix coefficients for the antenna on the right are known with respect to a coordinate system whose z-axis points outward rather than inward. 


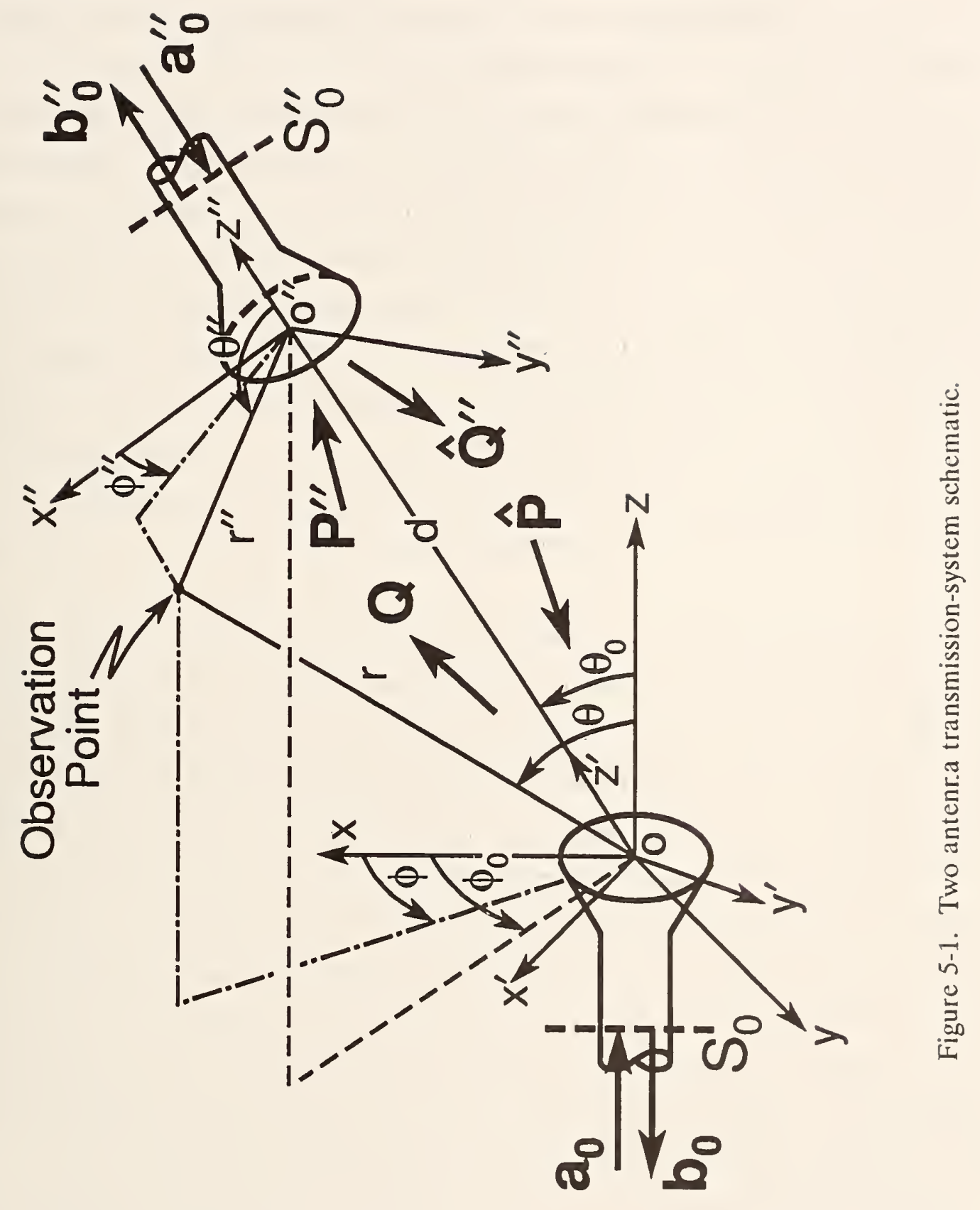


The location of a field point, the "observation point" in figure 5-1, can be specificd by any of the depicted coordinate systems. Thus, the observation point is denoted by $\mathrm{r}, \theta, \phi$, or by $\mathrm{r}^{\prime \prime}$, $\theta^{\prime \prime}, \phi^{\prime \prime}$ in the unprimed and doubly primed coordinate systems, respectively. The origin of coordinates in the doubly primed coordinate system is given the special location designation, $d$, $\theta_{0}, \phi_{0}$, with respect to the unprimed coordinate system.

An analysis of the scattering between the two antenna systems necessarily involves relating the spatial mode coefficients through a coordinate system transformation. That is, we transform the spatial functions describing the fields from the unprimed to the doubly primed coordinate system and vice versa. This coordinatc system transformation is broken up into two parts. The first part involves a pure rotation operation, resulting in parallel alignment of the Cartesian axes of the newly rotated coordinate system with the corresponding axes of the opposing antenna's coordinate system. The second part consists of rigid coordinate system translation until the transformed axes are coincident with the corresponding axes of the opposing-coordinate antenna system. Transforming in the opposite direction, one first performs a rigid coordinate system translation and then performs a pure rotation operation on the newly translated coordinate system.

The preceding discussion would include the general case in which the unprimed and doubly primed coordinate systems were each arbitrarily oriented in space. However, the application of our analysis to spherical scanning requires a particular orientation for the doubly primed coordinate system. For that analysis, the most convenient orientation occurs when the line connecting the origins of the unprimed and doubly primed coordinate systems is coincident with the $\mathrm{z}^{\prime \prime}$-axis. Besides being convenient for discussing spherical scanning, the translation of coordinates transformation is mathematically simpler for this configuration. Consequently, since spherical scanning is the major application of our analysis, we will assume that the translation of coordinates transformation is taken parallel to the $z^{\prime \prime}$-axis. This coincides with the setup depicted in figure 5-1. Our choice of the $\mathrm{z}^{\prime \prime}$-axis for the translation direction results in the singly primed coordinate system serving as an intermediate stage either when transforming from the doubly primed coordinate system to the unprimed coordinate system or vice versa.

A word or two concerning notation seems in order at this point. As suggested by the notation associated with the exciting and emergent mode coefficients in figure 5-1, double primes are appended to modal expansion coefficients, scattering-matrix parameters, and vector sphericalwavc functions which are associated with the doubly primed coordinate system, while a lack of primcs indicates association with the unprimed coordinate system. Also, similar quantities having 
just one prime will be associated with the singly primed coordinate system. We also need a means of distinguishing the scattering-matrix elements of one antenna system from the seatteringmatrix elements of the other antenna system when a coordinate system transformation has transformed the seattering matrix away from the original coordinate system into some other coordinate system, such as the singly primed coordinate system. Therefore, we shall adopt the convention that the elements of the scattering matrix for the doubly primed antenna system will have a circumflex (a hat) affixed to them to set them apart from the scattering-matrix elements of the unprimed antenna system, while the presence or absence of primes will indicate which coordinate system the scattering-matrix elements are referenced to. As an example, a modal receiving coefficient (see eq (3.2)) with a circumflex and a single prime would designate the receiving properties of the doubly primed antenna system with respect to an exciting mode in the singly primed coordinate system. The lack of just the circumflex on this same quantity would indieate that it was the modal reeeiving eoeffieient of the unprimed antenna system but once again with respect to an exciting mode in the singly primed coordinate system.

There is one clement in the spherical-wave source-scattering matrix that is unaffected by a eoordinate system transformation: the input reflection coefficient for the waveguide feed. The waveguide-feed modes are also unaffected by a coordinate system transformation. However, the input reflection coefficient is independent of coordinate system transformation only if the Hankel-Bessel function formulation is used to describe emergent and exciting modes; if the Hankel-Bessel function formulation is transformed to a Hankel-Hankel function formulation, for instance, that element of the transformed scattering matrix corresponding to the input reflection coefficient will no longer be independent of the coordinate-system transformation [36]-A.

The exciting and emergent modal-expansion coefficients express the fields due to the presence of both antenna systems and not just one of them; consequently, there is no real requirement either to place or not to place a circumflex above the modal-expansion coefficients. However, by placing a circumflex above the modal-expansion coefficients of fields that are radiating away from the doubly primed antenna system while omitting same from the modalexpansion coefficients of fields that are radiating away from the unprimed antenna system, we can introduce a much needed bit of redundaney into our expressions to clearly identify the direction of propagation. The use of this convention is illustrated in figure 5-1, which shows that both $\hat{Q}^{\prime \prime}$ and $\hat{\mathrm{P}}$ represent fields radiated or scattered from the doubly primed antenna system. Also, it may be seen from eq (3.1) that emergent $\left(\hat{Q}^{\prime \prime}\right)$ modal-expansion coefficients (with a 
circumflex) are proportional to $\hat{\mathrm{T}}^{\prime \prime}$ and/or $\hat{\mathrm{S}}^{\prime \prime}$, while emergent $(\mathrm{Q})$ modal-expansion coefficients (without a circumflex) are proportional to $\mathrm{T}$ and/or $\mathrm{S}$.

The two-antenna problem depicted in figure 5-1 presumes that the coupling between the antennas is not negligible. However, in treating spherical scanning in Chapter VIII it will be nccessary to assume that the antennas are far enough apart so that coupling effects are negligible, as otherwise the analysis becomes untractable. In order to see what is being neglected by this assumption, we shall present in this chapter a complete analysis of mutual coupling that fully accounts for the effects of multiple reflections between the two antenna systems.

Regarding treatises in which the effects of multiple reflections truly are negligible, Wasylkiwskyj and Kahn [57] present an exact expression for the mutual impedance betwcen canonical minimum-scattering antennas using sphcrical-wave scattering-matrix analysis. By definition of a canonical minimum-scattering antenna there is no scattered wave when the antenna ports are open-circuited [29], so the need for a multiple-reflection analysis is circumvented in their work. As there are no multiple reflections to deal with in their problem, the Hankel-Hankel function formulation for describing incoming and outgoing modes may readily be used without any disadvantage.

The solution to the problem of two mutually coupled antennas, as shown in figure 5-1, is cssentially obtained by an iterative technique in which successivcly highcr-order reflections between the two scattering objects (the antennas) are considered at each iterative stage. This process is mathematically equivalent to a Liouville-Ncumann series solution of the boundary value problcm [48]. One of the earliest treatments of multiple scattering using iterative techniques is due to Twcrsky [53], who considered the problem of scattering by an arbitrary configuration of parallcl cylinders. The numerical and experimental application of Twersky's analysis to the two-cylinder case was subsequently described by Row [45]. Moreover, the formal analysis devcloped by Twersky could be emulated to obtain an itcrative solution to the problem of threc or morc mutually coupled antennas using spherical scattcring-matrix representations. This could be of intcrest, say, when the effect of a scattering object located between a transmitting antenna and a receiving antenna needs to be accounted for.

The trcatment of multiple scattering by two perfectly conducting spheres was first presented by Liang and Lo [38]. Liang and Lo's analysis was extended to the problem of multiple scattcring between three spheres by Bruning and Lo [9], who also obtaincd a gcometrical-optics solution to the two-sphere problem. Bruning and Lo's ray-optical technique 
similarly involves multiple-reflection iterations, although it uses asymptotic solutions to the wave equation rather than exact modal solutions.

The earliest multiple-reflection iterative solution to the general problem of two coupled antennas was given by Kerns and Dayhoff [28], who used planar scattering-matrix analysis. Cylindrical scattering-matrix analysis giving a complete multiple-reflection solution to the problcm of two coupled antennas was first presented by Yaghjian [60]. The zeroth order term in his result had previously been obtained by Leach and Paris [33], who derived their result using the Lorentz reciprocity relationship [12]. As mentioned previously, this technique had been pioneered by Jensen [21] to study the coupling between two antennas using spherical coordinates, and it also resulted in a zeroth order term for the multiple-reflection problem. The approach taken by Wacker [56] to obtain an expression essentially equivalent to Jensen's result but without the constraint of reciprocity was to use spherical-wave scattering-matrix analysis, where the necessary modal transformations between the coordinate systems were carried out using grouprepresentation theory. The intent of his development was to transform just the modal receiving coefficients, as opposed to transforming all of the scattering-matrix elements as will be done here. Consequently, although both Wacker and the current work use group-representation techniques, the difference in emphasis results here in obtaining the complete multiple-reflection solution to the problem.

\subsection{Modal Coefficient-Transformation Solution to the Coupled Antenna Problem}

\subsubsection{Heuristic Development of the Two-Port Equations}

In order to treat the general problem of two coupled antennas shown in figure 5-1, we start by considering a much simpler case. In figure 5-2, we have the special situation where the respective $z$-axes of the two antennas are aligned, while the two remaining Cartesian axes are respectively parallel to their counterparts in the opposing coordinate system. This corresponds to letting the singly primed coordinate system represent the antenna on the left in figure 5-1. The goal of the current analysis is to first write down a solution for the situation depicted in figure 5-2 and then extend that result to the general case of figure 5-1.

The analysis of the antenna-coupling problem depicted in figure 5-2 follows from a straightforward extcnsion of expressing a coordinate system transformation as an inverse operation on the modal-expansion coefficients, as exemplified by eq (4.7). In order to be definite, we assume for the present that the antenna on the right in figure 5-2 (or in figure 5-1) is operating in the recciving mode, whilc the antenna on the left is opcrating in the transmitting 

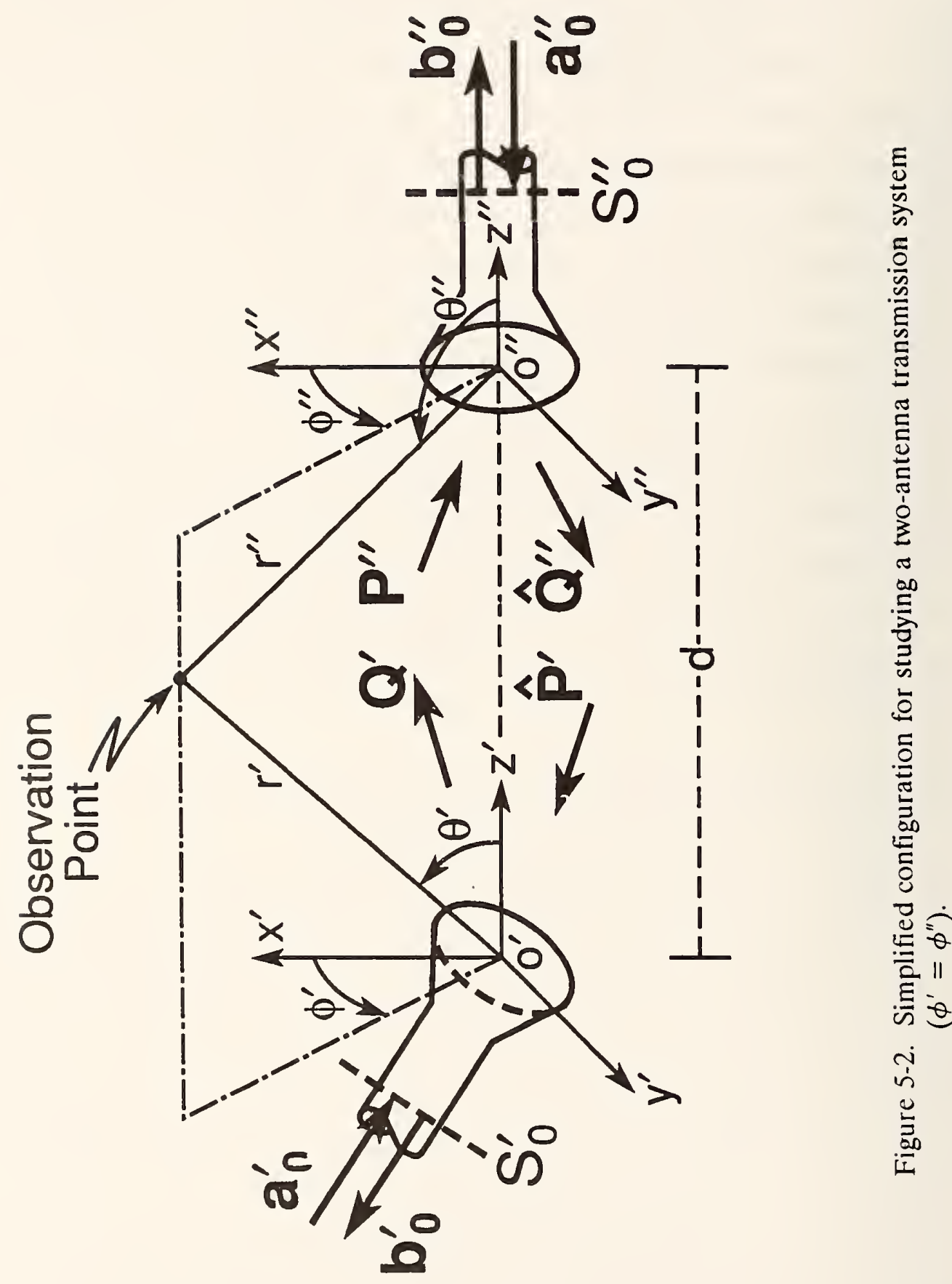
mode. Consequently, the equivalent generator-source wave [26, page 44] emitted into a non-reflecting load terminating the doubly-primed antenna system is, from eq (3.23),

$$
b_{G}^{\prime \prime}=\sum_{\sigma v \mu} \hat{R}_{\sigma \mu \nu}^{\prime \prime} P_{\sigma \mu \nu}^{\prime \prime},
$$

where the $\hat{R}_{\mathrm{o} \mu \nu}^{\prime \prime}$ represent the receiving coefficients for the doubly-primed antenna system, while the $P_{o \mu \nu}^{\prime \prime}$ are modal-expansion coefficients of the vector spherical-wave functions corresponding to the incident field. Now the field incident on the doubly primed antenna system is due to sources (or scatterers) in the singly primed coordinate system, which says in turn that the incident-field modes can be expressed as a linear combination of the emergent spherical-wave modes from the singly primed coordinate system. We shall establish this correspondence between the exciting spherical-mode expansion coefficients $P_{\mathrm{o} \mu \nu}^{\prime \prime}$ and a linear combination of the emergent spherical-mode expansion coefficients $Q^{\prime s u n}$ by developing common coordinate system expansions for the field emerging from the singly-primed antenna system and the field exciting the doubly primed antenna system. Then these two distinct field representations can be equated in their common region of validity to establish the desired correspondence.

Since the electromagnetic field vectors are invariant quantities under coordinate system transformations, and since the vector spherical-wave functions transform according to eq (4.26) in going from the singly primed to the doubly primed coordinate system, then the modal-expansion coefficients multiplying the vector spherical-wave functions must transform contragradiently to eq (4.26) in order to preserve the invariant character of the electromagnetic field structure. We express this with the equation

$$
P_{\sigma \mu \nu}^{\prime \prime}=\sum_{s n} C_{\sigma \mu \nu}^{s n} Q^{\prime s \mu n},
$$

where the transform coefficients $C_{\sigma \mu \nu}^{s n}$ will be expressed subsequently. Now we can substitute eq (5.2) into (5.1), interchange the order of summation, and upon making the definition

$$
\hat{R}_{s \mu n}^{\prime}=\sum_{\sigma \nu} C_{\sigma \mu \nu}^{s n} \hat{R}_{\sigma \mu \nu}^{\prime \prime}
$$

we obtain

$$
b_{G}^{\prime \prime}=\sum_{s n \mu} \hat{R}_{s \mu n}^{\prime} Q^{\prime s \mu n}
$$

The validity of interchanging the orders of summation will be discussed in the next section. Expression (5.3) represents a translation of the receiving coefficients $\hat{R}_{\sigma \mu \nu}^{\prime \prime}$ from the doubly 
primed to the singly primed coordinate system. The $C_{\sigma \mu v}^{s n}$ notation, incidentally, was originally introduced by Jensen [21] to express the translation of modal functions.

In writing eq (5.4) in place of (5.1), we have passed from an expression for the equivalent generator-source wave expressed in terms of the doubly primed coordinate system to an expression in the singly primed coordinate system. In order to pass from this result to an equivalent expression corresponding to the geometry of figure 5-1, we need to consider the effect on the emergent modal-expansion coefficients of a coordinate system rotation. Again, the electromagnetic field vectors must remain invariant, so eq (4.7) can be applied directly. Since the $z^{\prime}$-axis of the singly primed coordinate system is reached by a rotation $\phi_{0}, \theta_{0}$ from the unprimed coordinate system, we identify $\alpha, \beta$ in eq (4.7) with $\phi_{0}, \theta_{0}$ (see [44], Chapt. IV, figure 3 , p. 50 , and section 15 , pp. 62-67); then, upon admitting a rotation $\chi$ about the $\mathrm{z}^{\prime \prime}$-axis, wc obtain ${ }^{1}$

$$
Q^{\prime s \mu n}=\sum_{m} Q^{s i n n} D_{m \mu}^{n *}\left(\phi_{0}, \theta_{0}, \chi\right),
$$

where we have combined eqs (4.9) and (4.7), and where the asterisk denotes complex conjugation. Consequently, upon substituting eq (5.5) into (5.4) we have the result,

$$
b_{G}^{\prime \prime}=\sum_{s n \mu} \sum_{m} Q^{s m n} \hat{R}_{s \mu n}^{\prime} D_{m \mu}^{n *}\left(\phi_{0}, \theta_{0}, \chi\right) .
$$

A logical extcnsion of eq (5.4) is obtained by defining

whereupon cq (5.6) becomes

$$
\hat{R}_{s m n}=\sum_{\mu} \hat{R}_{s \mu n}^{\prime} D_{m \mu}^{n *}\left(\phi_{0}, \theta_{0}, \chi\right)
$$

$$
b_{G}^{\prime \prime}=\sum_{s n m} Q^{s m n} \hat{R}_{s m n}
$$

\subsubsection{Explicit Determination of the Translation Coefficients}

We now obtain an expression for the $C_{\sigma \mu \nu}^{s n}$. We start with the exprcssion for the electric field in the singly primed coordinate system, temporarily ignoring scattering by the receiving

${ }^{1}$ Hcre, rclating to the notation of Chapter IV, $Q^{1 \mathrm{mn}}=\mathrm{q}_{\mathrm{nn}}$, where these particular $\mathrm{q}_{\mathrm{nm}}$ coefficients are used with TE modes, whilc $\mathrm{Q}^{2 \mathrm{mn}}=\mathrm{q}_{\mathrm{n} n}$, where these particular $\mathrm{q}_{\mathrm{nn}}$ coefficients are used with $\mathrm{TM}$ modes. 
antenna. From eq (2.15a) we have

$$
\underline{E}^{\prime}=\underline{E}\left(r^{\prime}, \theta^{\prime}, \phi^{\prime}\right)=\sum_{n=1}^{\infty} \sum_{m=-n}^{n}\left(Q^{\prime 1 m n} \underline{M}_{m n}^{(1)^{\prime}}+Q^{\prime 2 m n} \underline{N}_{m n}^{(1)}\right), r^{\prime}<d .
$$

Now from eq (4.23) we substitute the translation transformation expressions for the vector spherical-wave functions into eq (5.7) to obtain

$$
\begin{aligned}
\underline{E}^{\prime}= & \sum_{n=1}^{\infty} \sum_{m=-n}^{n}\left\{Q^{\prime 1 m n} \sum_{v=(1,|m|)}^{\infty}\left(A_{m v}^{m n} \underline{M}_{m v}^{\prime \prime}+B_{m v}^{m n} \underline{N}_{m v}^{\prime \prime}\right)\right. \\
& \left.+Q^{\prime 2 m n} \sum_{v=(1,|m|)}^{\infty}\left(B_{m v}^{m n} \underline{M}_{m v}^{\prime \prime}+A_{m v}^{m n} \underline{N}_{m v}^{\prime \prime}\right)\right\} \\
= & \sum_{v=1}^{\infty} \sum_{m=-v}^{v}\left\{\sum_{n=(1,|m|)}^{\infty}\left(A_{m v}^{m n} Q^{\prime 1 m n}+B_{m v}^{m n} Q^{\prime 2 m n}\right) \underline{M}_{m v}^{\prime \prime}\right. \\
& \left.+\sum_{n=(1,|m|)}^{\infty}\left(B_{m v}^{m n} Q^{\prime 1 m n}+A_{m v}^{m n} Q^{\prime 2 m n}\right) \underline{N}_{m v}^{\prime \prime}\right\}
\end{aligned}
$$

We shall show in Chapter VI that interchanging the infinite $\mathrm{n}$ and $v$ summations is justified. For now, we just show that if interchanging the $\mathrm{n}$ and $v$ summation is justified, then the result indicated in eq (5.8) is indeed obtained. Thus, we have

$$
\begin{aligned}
\sum_{n=1}^{\infty} \sum_{m=-n}^{n} \sum_{v=(1,|m|)}^{\infty} & =\sum_{n=2}^{\infty} \sum_{v=1}^{n-1} \sum_{m=-v}^{v}+\sum_{n=1}^{\infty} \sum_{v=n}^{\infty} \sum_{m=-n}^{n} \\
& =\sum_{v=1}^{\infty} \sum_{n=v+1}^{\infty} \sum_{m=-v}^{v}+\sum_{v=1}^{\infty} \sum_{n=1}^{v} \sum_{m=-n}^{n} \\
& =\sum_{v=1}^{\infty} \sum_{m=-v}^{v} \sum_{n=v+1}^{\infty}+\sum_{v=1}^{\infty} \sum_{m=-v} \sum_{n=(1,|m|)}^{\infty} \\
& =\sum_{v=1}^{\infty} \sum_{m=-v}^{\infty} \sum_{n=(1,|m|)}^{\infty} .
\end{aligned}
$$

The derivation of eq (5.9) is easily visualized by two-dimensional index diagrams which exhibit the innermost-summation index's bounds as a function of the outer-summation index. 
Now an alternative expression for the electric field in the common region of validity is also obtaincd from eq (2.15a),

$$
\underline{E}^{\prime \prime}=\underline{E}\left(r^{\prime \prime}, \theta^{\prime \prime}, \phi^{\prime \prime}\right)=\sum_{\nu=1}^{\infty} \sum_{\mu=-v}^{\nu}\left(P_{1 \mu \nu}^{\prime \prime} \underline{M}_{\mu \nu}^{\prime \prime}+P_{2 \mu \nu}^{\prime \prime} \underline{N}_{\mu \nu}^{\prime \prime}\right), r^{\prime \prime}<d .
$$

Consequently, since $\underline{E}^{\prime}=\underline{E}^{\prime \prime}$ identically, we can equate like coefficients of the vector sphericalwave functions to obtain

$$
\begin{aligned}
& P_{1 \mu \nu}^{\prime \prime}=\sum_{n=(1,|\mu|)}^{\infty}\left(A_{\mu \nu}^{\mu n} Q^{\prime 1 \mu n}+B_{\mu \nu}^{\mu n} Q^{\prime 2 \mu n}\right) \\
& P_{2 \mu \nu}^{\prime \prime}=\sum_{n=(1,|\mu|)}^{\infty}\left(B_{\mu \nu}^{\mu n} Q^{\prime 1 m n}+A_{\mu \nu}^{\mu n} Q^{\prime 2 \mu n}\right) .
\end{aligned}
$$

Thus, eq (5.11) provides an implicit expression for the $C_{\sigma \mu \nu}^{s n}$ coefficients as defined by eq (5.2). We can express thesc coefficients in the form

$$
C_{\sigma \mu \nu}^{s n}=A_{\mu \nu}^{\mu n} \delta_{s \sigma}+B_{\mu \nu}^{\mu n} \delta_{s,[\sigma+1]_{2}},
$$

where the notation $[\sigma+1]_{2}$ is used to indicate that this quantity is to be taken cyclic in the integers 1,2. Thus, the Kronecker delta in eq (5.12) effectively becomes

$$
\delta_{s,[\sigma+1]_{2}}=\frac{1}{2}\left[1-(-)^{s+\sigma}\right] .
$$

Using eq (5.12) and the shorthand notation (2.45) for the vector spherical-wave functions we can rewrite the vector spherical-wave function addition theorem eq (4.23) in the compact form,

$$
\underline{F}_{s m n}^{(1)^{\prime}}=\sum_{\sigma=1}^{2} \sum_{\nu=(1,|m|)}^{\infty} C_{\sigma m \nu}^{s n} \underline{F}_{\sigma m v}^{\prime \prime}, r^{\prime \prime}<d,
$$

which expresses the translation transformation of both the $\underline{M}_{m n}^{(1)^{\prime}}$ and $\underline{\underline{N}}_{m n}^{(1)^{\prime}}$ functions. Except for vast differences in notation, eq (5.13) agrees perfectly with the translational addition theorems given by Liang and Lo [38, Appendix: eqs. A-11 and A-12]. However, the formulation presented in [21] for $C_{\sigma \mu \nu}^{s n}$ was apparently arrived at by taking the complex conjugate of just a portion of the translation transformation given by Liang and Lo and is consequently incorrect. 


\subsubsection{The Two-Port Equation for Oppositely Directed Transmission}

We now wish to consider the effect of taking the antenna on the right in figures 5-1 and 5-2 as the transmitting antenna while the antenna on the left becomes the receiving antenna, instead of vice versa. We start by considering the translation of the radiated field from the doubly primed coordinate system to the singly-primed coordinate system. Thus, once again ignoring scattering by the receiving antenna, we have the electric field in the doubly primed coordinate system from eq $(2.15 \mathrm{a})$,

$$
\begin{aligned}
& \underline{E}^{\prime \prime}=\sum_{v=1}^{\infty} \sum_{\mu=-v}^{v}\left(\hat{Q}^{\prime \prime 1 \mu v} \underline{M}_{\mu v}^{(1)^{\prime \prime}}+\hat{Q}^{\prime \prime 2 \mu v} \underline{N}_{\mu v}^{(1)^{\prime \prime}}\right), \quad r^{\prime \prime}<d \\
& =\sum_{v=1}^{\infty} \sum_{\mu=-v}^{v}\left\{\hat{Q}^{\prime \prime} 1 \mu v \sum_{n=(1,|\mu|)}^{\infty}\left(a_{\mu n}^{\mu v} \underline{M}_{\mu n}^{\prime}+b_{\mu n}^{\mu v} \underline{N}_{\mu n}^{\prime}\right)\right. \\
& \left.+\hat{Q}^{\prime \prime} 2 \mu v \sum_{n=(1,|\mu|)}^{\infty}\left(b_{\mu n}^{\mu v} \underline{M}_{\mu n}^{\prime}+a_{\mu n}^{\mu v} \underline{N}_{\mu n}^{\prime}\right)\right\} \\
& =\sum_{n=1}^{\infty} \sum_{\mu=-n}^{n}\left\{\sum_{v=(1,|\mu|)}^{\infty}\left(a_{\mu n}^{\mu \nu} \hat{Q}^{\prime \prime} 1 \mu \nu+b_{\mu \nu}^{\mu \nu} \hat{Q}^{\prime \prime 2 \mu \nu}\right) \underline{M}_{\mu n}^{\prime}\right. \\
& \left.+\sum_{v=(1,|\mu|)}^{\infty}\left(b_{\mu n}^{\mu v} \hat{Q}^{\prime \prime 1 \mu \nu}+a_{\mu n}^{\mu v} \hat{Q}^{\prime \prime 2 \mu v}\right) \underline{N}_{\mu n}^{\prime}\right\} \text {. }
\end{aligned}
$$

where the translation transformation eq (4.26) has been used, along with eq (5.9) to effect the interchange in the order of summation.

Now the electric field expansion in the singly primed coordinate system is given by

$$
\underline{E}^{\prime}=\sum_{n=1}^{\infty} \sum_{\mu=-n}^{n}\left(\hat{P}_{1 \mu n}^{\prime} \underline{M}_{\mu n}^{\prime}+\hat{P}_{2 \mu n}^{\prime} \underline{N}_{\mu n}^{\prime}\right), r^{\prime}<d .
$$

Consequently, equating the two expressions for the electric field in the common region of validity results in

$$
\begin{aligned}
& \hat{P}_{1 \mu n}^{\prime}=\sum_{v=(1,|\mu|)}^{\infty}\left(a_{\mu n}^{\mu v} \hat{Q}^{\prime \prime 1 \mu \nu}+b_{\mu n}^{\mu v} \hat{Q}^{\prime \prime 2 \mu v}\right) \\
& \hat{P}_{2 \mu n}^{\prime}=\sum_{v=(1,|\mu|)}^{\infty}\left(b_{\mu n}^{\mu v} \hat{Q}^{\prime \prime 1 \mu \nu}+a_{\mu n}^{\mu v} \hat{Q}^{\prime \prime 2 \mu \nu}\right)
\end{aligned} .
$$

This result can be rewritten in a manner analogous to eqs (5.2) and (5.12). Defining 


$$
\alpha_{s \mu n}^{\sigma \nu}=a_{\mu n}^{\mu \nu} \delta_{s \sigma}+b_{\mu n}^{\mu v} \delta_{s,[\sigma+1]_{2}},
$$

eq (5.16) becomes

$$
\hat{P}_{s \mu n}^{\prime}=\sum_{\sigma=1}^{2} \sum_{\nu=(1,|\mu|)}^{\infty} \alpha_{s \mu n}^{\sigma \nu} \hat{Q}^{\prime \prime \sigma \mu \nu} .
$$

From eq (5.18) it is seen that the $\hat{\mathrm{Q}}^{\prime \prime}$ coefficients transform into $\hat{\mathrm{P}}^{\prime}$ coefficients contragradiently as compared to the translation transformation eq (5.13) for the vector spherical-wave functions. Moreover, in analogy to eq (5.13), we can substitute the defining eq (5.17) into the eqs (4.26) to obtain

$$
\underline{F}_{\sigma \mu \nu}^{(1)^{\prime \prime}}=\sum_{s=1}^{2} \sum_{n=(1,|\mu|)}^{\infty} \alpha_{s \mu n}^{\sigma v} \underline{F}_{s \mu n}^{\prime} .
$$

Now referring to figure $5-1$, we wish to relate the singly primed modal-expansion coefficients to the unprimed modal-expansion coefficients. Thus, from eqs (4.7) and (4.8) we obtain

$$
\hat{P}_{s m n}=\sum_{\mu=-n}^{n} \hat{P}_{s \mu n}^{\prime} D_{m \mu}^{n}\left(\phi_{0}, \theta_{0}, \chi\right) .
$$

The generator-source wave (see eq (3.23)) produced in the waveguide feed to the unprimed antenna system is given by

$$
b_{G}=\sum_{s=1}^{2} \sum_{n=1}^{\infty} \sum_{m=-n}^{n} R_{s m n} \hat{P}_{s m n}
$$

where the $\mathrm{R}_{\mathrm{smn}}$ coefficients represent the unprimed antenna system's receiving properties. Therefore, it follows from eq (5.20) that

$$
b_{G}=\sum_{s=1}^{2} \sum_{n=1}^{\infty} \sum_{m=-n}^{n} R_{s m n} \sum_{\mu=-n}^{n} \hat{P}_{s \mu n}^{\prime} D_{m \mu}^{n}\left(\phi_{0}, \theta_{0}, \chi\right),
$$

or more compactly,

$$
b_{G}=\sum_{s n m} \sum_{\mu} R_{s m n} \hat{P}_{s \mu n}^{\prime} D_{m \mu}^{n}\left(\phi_{0}, \theta_{0}, \chi\right)
$$

where the $\hat{P}_{s \mu n}^{\prime}$ are translated emergent mode coefficients from the double-primed antenna system, as given by eq (5.18). 


\subsubsection{Antenna Coupling Expressed in Terms of Each Antenna's Spherical-Wave Source-Scattering-Matrix Elements}

We are now in position to collect results. First, we apply the defining expression (3.20) to eq (5.22) to obtain, upon changing index notation,

$$
b_{0}=\Gamma a_{0}+\sum_{s n m} \hat{P}_{s m n}^{\prime} \sum_{\mu} R_{s \mu n} D_{\mu m}^{n},
$$

where the arguments $\left(\phi_{0}, \theta_{0}, \chi\right)$ of $\mathrm{D}_{\mu \mathrm{m}}^{\mathrm{n}}$ are understood. We now reeall eq (3.3), whieh we reproduce here for eonvenience.

$$
Q^{s i n n}=T^{s i n n} a_{0}+\sum_{\sigma \nu \mu} S_{\sigma \mu \nu}^{s m n} \hat{P}_{\sigma \mu \nu} .
$$

Let us substitute eq (5.20) into eq (5.24) above and then substitute that result into eq (5.5) to obtain

$$
Q^{\prime s m n}=a_{0} \sum_{\mu} T^{s \mu n} D_{\mu m}^{n *}+\sum_{\sigma \nu \mu} \hat{P}_{\sigma \mu \nu}^{\prime} \sum_{m^{\prime} \mu^{\prime}} D_{m^{\prime} m}^{n *} S_{\sigma \mu^{\prime} \nu}^{s m^{\prime} n} D_{\mu^{\prime} \mu}^{\nu},
$$

where the arguments $\left(\phi_{0}, \theta_{0}, \chi\right)$ of $\mathrm{D}_{\mu \mathrm{m}}^{\mathrm{n}^{*}}$ and $\mathrm{D}_{\mu^{\prime} \mu}^{\mathrm{n}}$ have again been suppressed. Now from eçs (5.23) and (5.25) we ean express the souree-scattering-matrix elements for the unprimed antenna system with respect to the singly primed coordinate system. That is, we relate the source-scattering matrix elements of the antenna on the left in figure 5-1 through a coordinate system rotation to the souree-seattering matrix elements of the antenna on the left in figure 5.2. First, however, we note from eq (4.9) that $\left(D_{\mu \mathrm{m}}^{\mathrm{n}}\right)^{-1}=\mathrm{D}_{\mathrm{m \mu}}^{\mathrm{n}^{*}}$ where $\left(\mathrm{D}_{\mu \mathrm{n}}^{\mathrm{n}}\right)^{-1}$ is a rotation-matrix element for the inverse rotation $\left(-\chi_{0},-\theta_{0},-\phi_{0}\right)$. Now, the souree-scattering matrix equations for the antenna on the left in figure $5-1$ are

$$
\begin{gathered}
b_{0}=\Gamma^{\prime} a_{0}+\sum_{s n m} R_{s m n}^{\prime} \hat{P}_{s m n}^{\prime} \\
Q^{\prime s m n}=T^{\prime s m n} a_{0}+\sum_{\sigma \nu \mu} S_{\sigma \mu \nu}^{\prime s m n} \hat{P}_{\sigma \mu \nu}^{\prime} .
\end{gathered}
$$

Consequently,

$$
\begin{gathered}
\Gamma^{\prime}=\Gamma \\
R_{s m n}^{\prime}=\sum_{\mu=-n}^{n} R_{s \mu n} D_{\mu m}^{n} \\
T^{\prime \sin n}=\sum_{\mu=-n}^{n} D_{m \mu}^{n^{-1}} T^{s \mu n}
\end{gathered}
$$




$$
S_{\sigma \mu \nu}^{\prime s m n}=\sum_{m^{\prime}=-n}^{n} \sum_{\mu^{\prime}=-\nu}^{\nu} D_{m m^{\prime}}^{n^{-1}} S_{\sigma \mu^{\prime} \nu}^{s m^{\prime} n} D_{\mu^{\prime} \mu}^{\nu} .
$$

Equations (5.27) show the effect of a coordinate-system rotation on the elements of an antenna's source-scattering matrix. Similarly, we can determine the effect of a coordinate system translation on the source-scattering matrix elements. Specifically, we want to consider the effect of translating the source-scattering matrix of the doubly primed antenna (the antenna on the right in figures 5-1 and 5-2) from the doubly primed coordinate system into the singly primed coordinate system. The region of validity for representing the doubly primed antenna's field in terms of the singly primed coordinate system is a sphere centered about the singly primed coordinate-system whose radius falls just short of touching the doubly primed antenna. Note that the region of validity for the spatial modes related by eq (5.27) is the exterior of a spherical region, centered at the unprimed coordinate-system origin, that just encloses the unprimed antenna system. Consequently, we have a common region of validity between these two concentric spheres for describing the fields about each antenna.

The doubly primed antenna system's source-scattering matrix equations, with respect to the doubly primed coordinate system, are obtained from eqs (3.2) and (3.3) as

$$
\begin{gathered}
b_{0}^{\prime \prime}=\hat{\Gamma}^{\prime \prime} a_{0}^{\prime \prime}+\sum_{\sigma \nu \mu} \hat{R}_{\sigma \mu \nu}^{\prime \prime} P_{\sigma \mu \nu}^{\prime \prime} \\
\hat{Q}^{\prime \prime s m n}=\hat{T}^{\prime \prime s m n} a_{0}^{\prime \prime}+\sum_{\sigma \nu \mu} \hat{S}_{\sigma \mu \nu}^{\prime \prime s m n} P_{\sigma \mu \nu}^{\prime \prime} .
\end{gathered}
$$

The first of these two equations is just eq (5.1). We obtain the translated source-scattering matrix equations for the doubly primed antenna by substituting the second equation into eq (5.18) and then substituting eq (5.2) into the result. Upon applying eq (5.9) to interchange the orders of summation we obtain

$$
\begin{gathered}
b_{0}^{\prime \prime}=\hat{\Gamma}^{\prime} a_{0}^{\prime \prime}+\sum_{s n \mu} \hat{R}_{s \mu n}^{\prime} Q^{\prime s \mu n} \\
\hat{P}_{s t n}^{\prime}=\hat{T}^{\prime s t n} a_{0}^{\prime \prime}+\sum_{\sigma \nu \mu} \hat{S}_{\sigma \mu \nu}^{\prime s t n} Q^{\prime \sigma \mu \nu}
\end{gathered}
$$

where

$$
\hat{\Gamma}^{\prime}=\hat{\Gamma}^{\prime \prime}
$$




$$
\begin{aligned}
\hat{R}_{s \mu n}^{\prime} & =\sum_{p=1}^{2} \sum_{\nu=(1,|\mu|)}^{\infty} C_{p \mu \nu}^{s n} \hat{R}_{p \mu \nu}^{\prime \prime} \\
\hat{T}^{\prime} s t n & =\sum_{p=1}^{2} \sum_{\nu=(1,|t|)}^{\infty} \alpha_{s t n}^{p \nu} \hat{T}^{\prime \prime p t \nu} \\
\hat{S}_{\sigma \mu \nu}^{\prime s t n}= & \sum_{p=1}^{2} \sum_{r=(1,|t|)}^{\infty} \alpha_{s t n}^{p r} \sum_{\kappa=1}^{2} \sum_{\gamma=(1,|\mu|)}^{\infty} C_{\kappa \mu \gamma}^{\sigma \nu} \hat{S}_{\mathrm{\kappa} \mu \gamma}^{\prime \prime p t r} .
\end{aligned}
$$

Here, the notation $(1,|\mu|)$ designates the larger of 1 or $|\mu|$. Note that eq $(5.30 \mathrm{~b})$ is the same expression as eq (5.3). Further details for obtaining eq (5.30c) and eq (5.30d) are presented at the end of the next chapter.

Equations (5.26) and (5.29) represent pairs of coupled equations, referenced to a common coordinate system, which can be solved simultaneously for the mutual coupling between the two antennas of figure 5-2. However, it would be more useful to obtain a solution in terms of the setup depicted in figure 5-1. Accordingly, we need to invert the coordinate-system rotation transformation eq (5.27) and apply that result to eq (5.30) in order to obtain a combined translation-rotation transformation of the doubly primed antenna system's source-scattering matrix. Inverting eq (5.27), we obtain upon using eq (4.8) the expressions

$$
\begin{gathered}
\hat{R}_{s m n}=\sum_{\mu=-n}^{n} \hat{R}_{s \mu n}^{\prime} D_{\mu m}^{n^{-1}} \\
\hat{T}^{s m n}=\sum_{\mu=-n}^{n} D_{m \mu}^{n} \hat{T}^{\prime s \mu n} \\
\hat{S}_{\sigma \mu \nu}^{s m n}=\sum_{m^{\prime}=-n}^{n} \sum_{\mu^{\prime}=-\nu}^{v} D_{m m^{\prime}}^{n}, \hat{S}_{\sigma \mu^{\prime} \nu}^{\prime s m^{\prime} n} D_{\mu^{\prime} \mu}^{\nu^{-1}} .
\end{gathered}
$$

Substituting eq (5.30) into the above, we obtain

$$
\begin{aligned}
& \hat{R}_{s m n}=\sum_{p=1}^{2} \sum_{\mu=-n}^{n} \sum_{\nu=(1,|\mu|)}^{\infty} \hat{R}_{p \mu \nu}^{\prime \prime} C_{p \mu \nu}^{s n} D_{\mu m}^{n^{-1}} \\
& \hat{T}^{s m n}=\sum_{p=1}^{2} \sum_{\mu=-n}^{n} \sum_{\nu=(1,|\mu|)}^{\infty} D_{m \mu}^{n} \alpha_{s \mu n}^{p \nu} \hat{T}^{\prime \prime p \mu \nu}
\end{aligned}
$$




$$
\hat{S}_{\sigma \mu \nu}^{s m n}=\sum_{p=1}^{2} \sum_{t=-n}^{n} \sum_{r=(1,|t|)}^{\infty} D_{m t}^{n} \alpha_{s t n}^{p r} \sum_{\kappa=1}^{2} \sum_{\zeta=-\nu}^{\nu} \sum_{\gamma=(1,|\zeta|)}^{\infty} \hat{S}_{\kappa \zeta \gamma}^{/ / p t r} C_{\kappa \zeta \gamma}^{\sigma \nu} D_{\zeta \mu}^{\nu^{-1}} .
$$

The souree-seattering matrix equations for the doubly primed antenna system, with respect to the unprimed coordinate system, are

$$
\begin{aligned}
b_{0}^{\prime \prime} & =\hat{\Gamma}^{\prime \prime} a_{0}^{\prime \prime}+\sum_{s n m} \hat{R}_{s m n} Q^{s m n} \\
\hat{P}_{s m n} & =\hat{T}^{s m n} a_{0}^{\prime \prime}+\sum_{\sigma \nu \mu} \hat{S}_{\sigma \mu \nu}^{s m n} Q^{\sigma \mu \nu} .
\end{aligned}
$$

We are now in position to substitute eq (5.32b) into (5.24) and then solve the resulting expression for the $\mathrm{Q}^{\mathrm{smn}}$ modal-expansion coeffieients via a Liouville-Newmann series expansion (method of successive approximations). That is, we have to solve the expression

$$
Q^{\sin n}=T^{\sin n} a_{0}+\sum_{\sigma \nu \mu} S_{\sigma \mu \nu}^{s m n} \hat{T}^{\sigma \mu \nu} a_{0}^{\prime \prime}+\sum_{\sigma \nu \mu} S_{\sigma \mu \nu}^{s m n} \sum_{p r t} \hat{S}_{p t r}^{\sigma \mu \nu} Q^{p t r} .
$$

The series solution of equation (5.33) is most easily visualized by reeasting it in terms of a matrix equation. Thus, for the particular case $a_{0}^{\prime \prime}=0$, equation (5.33) can be written as

$$
\underline{Q}=\underset{\sim}{T} a_{0}+\underset{z}{Q} \underset{\sim}{ }
$$

where $Q$ represents the column matrix of unknown coefficients $Q^{\mathrm{smn}}, T$ the column matrix of the antenna transmission eharaeteristies $T^{\text {smn }}$, and $\underset{\approx}{K}$ designates a square matrix. The solution to the set of coupled simultaneous equations (5.34) may be expressed as

$$
Q=(\underset{\approx}{I}-\underset{\approx}{K})^{-1} \underset{\sim}{T} a_{0}=\underset{\sim}{T} a_{0}+\underset{\approx}{K} \underset{\sim}{T} a_{0}+\underset{\sim}{K^{2}} \underset{\sim}{T} a_{0}+\underset{\approx}{K^{3}} \underset{\sim}{T} a_{0}+\cdots .
$$

Note that each successive term in this series corresponds to successive round-trip multiple reflections between the transmitting and receiving antennas.

We eomplete this analysis by ealeulating the seattering matrix of the system two-port, which is defined in [25] by the equations

$$
\begin{aligned}
& b_{0}=M_{11} a_{0}+M_{12} a_{0}^{\prime \prime} \\
& b_{0}^{\prime \prime}=M_{21} a_{0}+M_{22} a_{0}^{\prime \prime}
\end{aligned}
$$

We obtain the two-port matrix elements by sueeessive elimination of the $\mathrm{P}_{\mathrm{smn}}$ and $\mathrm{Q}^{\mathrm{smn}}$ modalexpansion eoeffieients among eqs (5.21), (5.24), and (5.32), using eq (3.20) with (5.21) to get the desired format. The proeess indieated by eq (5.35) produces the following expressions when the Liouville-Neumann series solution is truncated at the third term. 


$$
\begin{aligned}
& M_{11}=\Gamma+\sum_{s n m} R_{s m n} \sum_{\sigma v \mu} \hat{S}_{\sigma \mu v}^{s m n}\left(T^{\sigma \mu \nu}+\sum_{p \xi u} S_{p u \xi}^{\sigma \mu v} \sum_{\kappa \gamma \zeta} \hat{S}_{x \zeta \gamma}^{p u \xi}\left(T^{\mathrm{\alpha} \zeta \gamma}+\sum_{q r t} S_{q t r}^{\mathrm{k} \zeta \gamma} \sum_{h \ell k} \hat{S}_{h k \ell}^{q q r} T^{h k \ell}\right)\right) \\
& M_{12}=\sum_{s n m} R_{s m n}\left(\hat{T}^{s m n}+\sum_{\sigma \vee \mu} \hat{S}_{\sigma \mu \nu}^{s m n} \sum_{p \xi u} S_{p u \xi}^{\sigma \mu v}\left(\hat{T}^{p u \xi}+\sum_{\kappa \gamma \zeta} \hat{S}_{x \zeta \zeta}^{p u \xi} \sum_{q r t} S_{q t r}^{\alpha \zeta \gamma} \hat{T}^{q t r}\right)\right) \\
& M_{21}=\sum_{s n m} \hat{R}_{s m n}\left(T^{s m n}+\sum_{o v \mu} S_{o \mu v}^{s m n} \sum_{p \xi u} \hat{S}_{p u \xi}^{o \mu v}\left(T^{p u \xi}+\sum_{\kappa \gamma \zeta} S_{\kappa \zeta \gamma}^{p u \xi} \sum_{q r t} \hat{S}_{q t r}^{\kappa \zeta \gamma} T^{q t r}\right)\right) \\
& M_{22}=\hat{\Gamma}^{\prime \prime}+\sum_{s n m} \hat{R}_{s m n} \sum_{\sigma v \mu} S_{\mathrm{o} \mu \nu}^{s m n}\left(\hat{T}^{\sigma \mu \nu}+\sum_{p \xi u} \hat{S}_{p u \xi}^{\mathrm{o \mu \nu}} \sum_{\mathrm{k \gamma \zeta}} S_{\mathrm{k} \zeta \gamma}^{p u \xi}\left(\hat{T}^{\mathrm{k} \zeta \gamma}+\sum_{q r t} \hat{S}_{q t r}^{\mathrm{\zeta} \zeta \gamma} \sum_{h \ell k} S_{h k \ell}^{q r r} \hat{T}^{h k \ell}\right)\right) .
\end{aligned}
$$

The preceding expressions for the system two-port can be used when one antenna acts as a passive scatterer, or else to evaluate a multiple-reflection eomponent between a transmitting and a receiving antenna. An interesting cxtension to this analysis would be to account for a third scattering objeet using the iterative technique of Twersky [53], as pointed out in section 5.1. In the remainder of the current work, howcver, it will be assumed that all cffects due to multiple reflections can be neglected; consequently our interest will center on the applications of cqs (5.6) and (5.22).

\subsection{Conclusions}

In this chapter we developed the formulation for expressing the mutual coupling between two antennas, using the souree-seattering matrix deseription developed in Chapter III to describe each antcnna. The mutual eoupling betwecn these antennas is cxpresscd in terms of the functional transformations introduced in Chapter IV to describe the effects of coordinate system rotation and translation. We go on to present a transformed source-scattcring matrix represcntation of onc of the two coupled antennas in terms of the other antenna's preferred coordinate system (eqs (5.31)). The chapter is then concluded with the devclopment of a Liouville-Neumann series expansion (eq (5.35)) for the elements of the system's two-port scattering matrix (eq (5.36)). Thr result is given in equations (5.37) as a series of multiplereflcction terms truncatcd at the third multiple reflection.

In the next chapter we consider interrclations between the two reflectionless transmission expressions (eq (5.6) and eq (5.22)) developed up to this point. 


\section{CHAPTER VI}

\section{INTERRELATIONS BETWEEN THE TRANSMISSION EQUATIONS}

\subsection{Introduction}

As indicated at the end of the preceding chapter, our principal application of the system two-port equations occurs when multiple reflections between the two antennas can be neglected. We have obtained two apparently diverse expressions ((5.6) and (5.22)) for reflectionless transmission between two antennas, depending upon the transmission direction. In this chapter we show the similarities between these two expressions and then go on to see what happens when one or both antennas are reciprocal. If both antennas are reciprocal, then we will show that the two oppositely directed transmission formulas are equivalent. As a by-product, we will show that we only need to work with one of the two transmission formulas in order to develop a general algorithm for obtaining far-field antenna patterns using near-zone spherical scanning, regardless of the transmission direction and regardless of whether the test antenna is reciprocal, provided that the probe used for collecting near-field data is itself reciprocal. We also relate the unknown receiving-probe coefficients, corresponding to the probe's measurement orientation, to the probe's transmitting coefficients, where the latter are obtained from an independent measurement. Finally, using the same analytical expressions that established similarities between the two oppositely directed transmission formulas, we shall show that interchanging the $\mathrm{n}$ and $v$ summations in eq (5.9) is valid. The summation formula (5.9) is used for establishing the two oppositely directed transmission formulas, as well as establishing the source-scattering matrix translation formulas and the multiple reflection solution to the two-antenna coupling problem. Consequently, multiple rcflections are treated as a part of establishing the validity of interchanging the summation indexes in eq (5.9). As a by-product of doing this, we also obtain a coordinate-system translation theorem for the dyadic Green's function.

\subsection{Establishment of Equalities between the Contragradient Vector-Wavefunction Translation Coefficients}

Equations (5.3) and (5.6) together relate the doubly primed antenna's receiving coefficients and the unprimed antcnna's radiated-field modal-expansion coefficients to give the received generator-sourcc wave at the terminals of the doubly-primed antenna system. Combining these 
two expressions, we obtain for the received generator-source wave

$$
b_{G}^{\prime \prime}=\sum_{s=1}^{2} \sum_{n=1}^{\infty} \sum_{m=-n}^{n} Q^{s m n} \sum_{\mu=-n}^{n}\left[\sum_{\sigma=1}^{2} \sum_{\nu=(1,|\mu|)}^{\infty} C_{\sigma \mu \nu}^{s n} \hat{R}_{\sigma \mu \nu}^{\prime \prime}\right] D_{m \mu}^{n *}\left(\phi_{0}, \theta_{0}, \chi\right) .
$$

Similarly, when the roles of the transmitting and recciving antennas arc interchanged, we obtain from eqs (5.18) and (5.22) the result

$$
b_{G}=\sum_{s=1}^{2} \sum_{n=1}^{\infty} \sum_{m=-n}^{n} R_{s m n} \sum_{\mu=-n}^{n}\left[\sum_{\sigma=1}^{2} \sum_{\nu=(1,|\mu|)}^{\infty} \alpha_{s \mu n}^{\sigma \nu} \hat{Q}^{\prime \prime \sigma \mu \nu}\right] D_{m \mu}^{n}\left(\phi_{0}, \theta_{0}, \chi\right) .
$$

Both of the prcceding cxpressions are required in order to describe the received signal when nonreciprocal antennas are used. An antenna might be nonreciprocal due to the presence of ferrite phase-shifting clements subject to a static-magnetic biasing field [25], for instance.

Howcver, for the very large class of antennas that are reciprocal, the received generator-source wave can be expected to bc independent of the direction of transmission.

In this section we establish an additional mathematical identity relating the vector spherical-wave function translation coefficients used in eqs (6.1) and (6.2). In the next section these mathematical identities will be used to establish equivalences between these two transmission formulas in the case of reciprocal antennas.

Let us start by considering the translation coefficients in eq (6.2). From eq (5.19) we recall that these coefficients are used in translating the vector spherical-wave functions from the singly primed to the doubly primed coordinate system. Rewriting eq (4.26) to conform with the notation used in eq (5.19) we have

$$
\underline{M}_{\mu \nu}^{(1)^{\prime \prime}}=\sum_{n=(1,|\mu|)}^{\infty}\left(a_{\mu n}^{\mu \nu} \underline{M}_{\mu n}^{\prime}+b_{\mu n}^{\mu \nu} \underline{N}_{\mu n}^{\prime}\right), r^{\prime}<d
$$

and

$$
\underline{N}_{\mu \nu}^{(1)^{\prime \prime}}=\sum_{n=(1,|\mu|)}^{\infty}\left(a_{\mu n}^{\mu \nu} \underline{N}_{\mu n}^{\prime}+b_{\mu n}^{\mu \nu} \underline{M}_{\mu n}^{\prime}\right), r^{\prime}<d
$$

Expressions for the translation coefficients in eq (6.3) are obtained from eqs (4.24) and (4.27) as

$$
\begin{array}{r}
a_{\mu n}^{\mu \nu}=(-)^{\mu} i^{\nu-n} \frac{n+1 / 2}{n(n+1)} \sqrt{\frac{(v-\mu) !(n+\mu) !}{(v+\mu) !(n-\mu) !} \sum_{p} i^{p}[n(n+1)} \\
+v(v+1)-p(p+1)] a(\mu, v,-\mu, n, p) h_{p}^{(1)}(k d)
\end{array}
$$

and

$$
b_{\mu n}^{\mu \nu}=(-)^{\mu} i^{\nu-n-1}(\mu k d) \frac{2 n+1}{n(n+1)} \sqrt{\frac{(v-\mu) !(n+\mu) !}{(v+\mu) !(n-\mu) !}} \sum_{p} i^{p} a(\mu, v,-\mu, n, p) h_{p}^{(1)}(k d),
$$


where

$$
a(\mu, v,-\mu, n, p)=(2 p+1) \sqrt{\frac{(v+\mu) !(n-\mu) !}{(v-\mu) !(n+\mu) !}}\left(\begin{array}{ccc}
v & n & p \\
0 & 0 & 0
\end{array}\right)\left(\begin{array}{ccc}
v & n & p \\
\mu & -\mu & 0
\end{array}\right) \text {. }
$$

It is of special interest to note that the square-root factors in eqs (6.4) are the reciprocal of the square-root factor in eq (6.5). In eq (6.5), the quantities

$$
\left(\begin{array}{lll}
j_{1} & j_{2} & j_{3} \\
m_{1} & m_{2} & m_{3}
\end{array}\right)
$$

are the Wigner 3-j symbols [17]. Edmonds [17] has shown, for $m_{1}=m_{2}=m_{3}=0$, that the Wigner 3-j symbol (eq (6.6)) is equal to zero unless the sum, $\mathrm{j}_{1}+\mathrm{j}_{2}+\mathrm{j}_{3}$, is even. Assuming this sum is even, then symmetry relations [17] along the Wigner 3-j symbols show that

$$
\left(\begin{array}{lll}
v & n & p \\
0 & 0 & 0
\end{array}\right)=\left(\begin{array}{lll}
n & v & p \\
0 & 0 & 0
\end{array}\right)
$$

and

$$
\left(\begin{array}{ccc}
v & n & p \\
\mu & -\mu & 0
\end{array}\right)=\left(\begin{array}{ccc}
n & v & p \\
-\mu & \mu & 0
\end{array}\right)=\left(\begin{array}{ccc}
n & v & p \\
\mu & -\mu & 0
\end{array}\right)
$$

Consequently, combining eqs (6.7) and (6.8) in (6.5) we readily obtain

$$
a(\mu, v,-\mu, n, p)=\frac{(\nu+\mu) !(n-\mu) !}{(\nu-\mu) !(n+\mu) !} a(\mu, n,-\mu, v, p)=a(-\mu, n, \mu, v, p) \text {. }
$$

Next, we contrast the translation algorithm (eq (6.3)) with eq (4.23), the algorithm for translating vector spherical-wave functions from the doubly primed to the singly primed coordinate system. The translation coefficients for this oppositely directed translation algorithm are given by eq (4.24), which we reproduce here for convenience of comparison with eq (6.4). Thus, the translation coefficients contragradient to the coefficients in eq (6.3) are given by

$$
\begin{aligned}
& A_{\mu \nu}^{\mu n}=(-)^{\mu} i^{v-n} \frac{v+1 / 2}{v(v+1)} \sqrt{\frac{(v+\mu) !(n-\mu) !}{(v-\mu) !(n+\mu) !} \sum_{p} i^{p}[n(n+1)} \\
&+v(v+1)-p(p+1)] a(\mu, n,-\mu, v, p) h_{p}^{(1)}(k d)
\end{aligned}
$$

and

$$
\begin{aligned}
& B_{\mu \nu}^{\mu n}=(-)^{\mu} i^{v-n+1}(\mu k d) \frac{2 v+1}{v(v+1)} \sqrt{\frac{(v+\mu) !(n-\mu) !}{(v-\mu) !(n+\mu) !}} \\
& \times \sum_{p} i^{p} a(\mu, n,-\mu, v, p) h_{p}^{(1)}(k d) .
\end{aligned}
$$


We can now express the equalities between the contragradient vector sphericalwavefunction translation coefficients (6.4) and (6.10). Thus, using eq (6.9), we obtain the relations

$$
a_{\mu n}^{\mu \nu}=\frac{2 n+1}{n(n+1)} \times \frac{\nu(\nu+1)}{2 \nu+1} A_{\mu \nu}^{\mu n}, \quad b_{\mu n}^{\mu \nu}=-\frac{2 n+1}{n(n+1)} \times \frac{\nu(\nu+1)}{2 \nu+1} B_{\mu \nu}^{\mu n}
$$

and

$$
a_{-\mu, n}^{-\mu, v}=a_{\mu n}^{\mu \nu}, \quad b_{-\mu, n}^{-\mu, v}=-b_{!, n}^{\mu \nu} .
$$

Using eqs (6.11) and (6.12) with the definitions (5.12) and (5.17), we obtain the desired relationship between the translation coeffieients in eqs (6.1) and (6.2). Thus, we have

$$
\alpha_{s \mu n}^{\sigma v}=\frac{2 n+1}{n(n+1)} \frac{v(v+1)}{2 v+1} C_{\sigma,-\mu, v}^{s n} .
$$

From this relationship, we can re-express eq (5.18), for instance, in the form

$$
\hat{P}_{s \mu n}^{\prime}=\frac{2 n+1}{n(n+1)} \sum_{\sigma \nu} \frac{\nu(\nu+1)}{2 \nu+1} C_{\sigma,-\mu, \nu}^{s n} \hat{Q}^{\prime \prime \sigma \mu \nu} .
$$

Similarly, we could re-express eq (5.3) using $\frac{2 v+1}{v(v+1)} \frac{n(n+1)}{2 n+1} \alpha_{s,-\mu, n}^{o v}$ in place of $C_{\sigma \mu \nu}^{s n}$. Note that cqs (6.13) and (6.14) apply irrespeetive of any reeiprocity eonstraints on the antennas depieted in figure $5-2$.

\subsection{Equivalence of the Coupling Equations Representing Oppositely Directed Transmission for the Case of Reciprocal Antennas}

We now consider the application of eq (6.13) to the particular situation where the scattering matrix of the doubly primed antenna system satisfies the reeiproeity relation (3.8). To be definite, we assume that the doubly primed antenna is operating in the transmitting mode, while the unprimed antenna functions as a reeeiver. We further assume that the contribution of the incoming modal coefficients (5.2) to the outgoing model coefficients is negligible, so eq $(5.28 \mathrm{~b})$ reduces to

$$
\hat{Q}^{\prime \prime \sigma \mu \nu}=\hat{T}^{\prime \prime \sigma \mu \nu} a_{0}^{\prime \prime} .
$$

Consequently, plugging eq (6.15) into (6.14) and using the reeiprocity relationship (3.8) results in

$$
\begin{aligned}
\hat{P}_{s \mu n}^{\prime} & =a_{0}^{\prime \prime} \frac{\hat{\eta}_{0}^{\prime \prime} k^{2}}{2 \pi \eta}(-)^{\mu} \frac{2 n+1}{n(n+1)} \sum_{\sigma \nu} C_{\sigma,-\mu, \nu}^{s n} \hat{R}_{\sigma,-\mu, \nu}^{\prime \prime} \\
& =a_{0}^{\prime \prime} \frac{\hat{\eta}_{0}^{\prime \prime} k^{2}}{2 \pi \eta}(-)^{\mu} \frac{2 n+1}{n(n+1)} \hat{R}_{s,-\mu, n}^{\prime},
\end{aligned}
$$

where we have used eq (5.3) to simplify the final result in eq (6.16). 
Reversing the order of summation of the $m$ and $\mu$ indices in eq (5.22) results in

$$
b_{G}=\sum_{s n m} \sum_{\mu} R_{s,-m, n} \hat{P}_{s,-\mu, n}^{\prime} D_{-m,-\mu}^{n}\left(\phi_{0}, \theta_{0}, \chi\right) .
$$

Now we ean readily obtain [44] the expression

$$
D_{-m,-\mu}^{n}\left(\phi_{0}, \theta_{0}, \chi\right)=(-)^{m-\mu} D_{m \mu}^{n *}\left(\phi_{0}, \theta_{0}, \chi\right) \text {. }
$$

Consequently, substituting eqs (6.16) and (6.18) into (6.17) results in

$$
b_{G}=a_{0}^{\prime \prime} \frac{\hat{\eta}_{0}^{\prime \prime} k^{2}}{2 \pi \eta} \sum_{s n m}(-)^{m} \frac{2 n+1}{n(n+1)} R_{s,-m, n} \sum_{\mu} \hat{R}_{s \mu n}^{\prime} D_{m, \mu}^{n *}\left(\phi_{0}, \theta_{0}, \chi\right) .
$$

If the unprimed antenna is also reeiproeal, we ean apply eq (3.8) onee again to obtain

$$
b_{G}=\frac{\hat{\eta}_{0}^{\prime \prime} a_{0}^{\prime \prime}}{\hat{\eta}_{0}} \sum_{s n m} T^{s m n} \sum_{\mu} \hat{R}_{s \mu n}^{\prime} D_{m, \mu}^{n *}\left(\phi_{0}, \theta_{0}, \chi\right) \text {. }
$$

Equation (6.20) is obtained for transmission from the doubly primed antenna to the unprimed antenna by making the assumption that both antennas are reeiproeal. We ean now compare this result with that obtained for transmission in the opposite direction, as expressed by eq (5.6) or (6.1). In this case, the eontribution of the refleeted signal (5.20) or (5.18) from the doubly primed antenna system is assumed to contribute negligibly to re-radiated modes from the unprimed antenna system, so eq (5.24) reduces to $\mathrm{Q}^{\mathrm{smn}}=\mathrm{a}_{0} \mathrm{~T}^{\mathrm{smn}}$. Therefore, eq (5.6) ean be written as

$$
b_{G}^{\prime \prime}=a_{0} \sum_{s n m} T^{s m n} \sum_{\mu} \hat{R}_{s \mu n}^{\prime} D_{m, \mu}^{n *}\left(\phi_{0}, \theta_{0}, \chi\right) .
$$

Comparing this with eq $(6.20)$, we see that we have the reeiproeity relation

$$
b_{G}^{\prime \prime}=\frac{a_{0} \hat{\eta}_{0}}{a_{0}^{\prime \prime} \hat{\eta}_{0}^{\prime \prime}} b_{G}
$$

We next need to eheek on the result of starting with eq (6.21) instead of (5.22) in applying the reeiproeity relationships. If we at first simply assume that just the doubly primed antenna system is reeiprocal, then with the unprimed antenna system operating in the transmitting mode and the doubly primed antenna system operating in the reeeiving mode, we ean apply eqs (6.13), (6.18), and the reeiprocity relation (3.8) to eq (6.21) to obtain

$$
b_{G}^{\prime \prime}=\frac{2 \pi \eta}{\hat{\eta}_{0}^{\prime \prime} k^{2}} a_{0} \sum_{s n m}(-)^{m} \frac{n(n+1)}{2 n+1} T^{s,-m, n} \sum_{\mu}\left(\sum_{\sigma v} \alpha_{s \mu n}^{\sigma v} \hat{T}^{\prime \prime} \sigma \mu v\right) D_{m \mu}^{n}\left(\phi_{0}, \theta_{0}, \chi\right) .
$$

If the unprimed antenna system is also reeiproeal, then eq (3.8) can onee again be applied, resulting in the expression 


$$
b_{G}^{\prime \prime}=\frac{\hat{\eta}_{0} a_{0}}{\hat{\eta}_{0}^{\prime \prime}} \sum_{s n m} R_{s m n} \sum_{\mu}\left(\sum_{\sigma \nu} \alpha_{s \mu n}^{\sigma \nu} \hat{T}^{\prime \prime \sigma \mu \nu}\right) D_{m \mu}^{n}\left(\phi_{0}, \theta_{0}, \chi\right) .
$$

Consequently, upon comparing eq (6.24) with (6.2), we obtain again the reciprocity relation (6.22). Thus, we have demonstrated that the available signal delivered to a receiver by the transmission system depicted in figure 5-1 is independent of the direction of transmission when the antennas are reciprocal, provided that the excitation amplitudes and waveguide admittances are identical for both antennas.

\subsection{Application of the Transmission Equations to Near-Field to Far-Field Antenna Pattern Transformations (Spherical Scanning)}

\subsubsection{Equivalence of the Transmitting Test-Antenna and Receiving Test-Antenna Spherical- Scanning Computer Algorithms}

At this point, we consider the application of the transmission equations to spherical scanning, that is, to the problem of determining the far-field antenna pattern given the measured near-field antenna pattern. This topic will be treated in detail for the case of a transmitting test antenna and a receiving probe in Chapter VII, based upon the transmission formula (6.1). Here, we wish to answer the question of whether we can limit our development to just using eq (6.1), or whether we nced a second spherical-scanning algorithm, based upon the transmission formula (6.2), in order to handle a nonreciprocal receiving test antenna.

The starting point for developing a transmitting test antenna, probe compensated, spherical-scanning algorithm is given by eq (5.6) as

$$
b_{G}^{\prime \prime}=\sum_{s n m} Q^{s m n} \sum_{\mu} \hat{R}_{s \mu n}^{\prime} e^{i m \phi} d_{m, \mu}^{n}(\theta) e^{i \mu \chi},
$$

where we have substituted eq (4.2) for the element $D_{m \mu}^{n}(\phi, \theta, \chi)$ of the rotation coefficient matrix, and where the zero subscripts on the Euler angles $\theta$ and $\phi$ have been suppressed.

We will subsequently show how orthogonality relations can be used to dccouple the twoterm sum over s, $\sum_{s=1}^{2} Q^{s m n} \hat{R}_{s \mu n}^{\prime}$. Then, if the translated probe receiving coefficients $\hat{R}_{s \mu n}^{\prime}$ are known, we can solve for the unknown modal-expansion coefficients $\mathrm{Q}^{\mathrm{smn}}$. Oncc these modal expansion coefficients are known, then the far-field pattcrn can be obtained from eq (6.25) by replacing the translated probe receiving coefficients by translated far-field ideal-dipole recciving coefficients. The details underlying this overly brief description arc given in Chapter VIII. 
The equation for the receiving test-antenna case to be solved in a manner analogous to the treatment of eq (6.25) is given by eq (5.22) as

$$
b_{G}=\sum_{s n m} R_{s m n} \sum_{\mu} \hat{P}_{s \mu n}^{\prime} e^{-i m \phi} d_{m \mu}^{n}(\theta) e^{-i \mu \chi},
$$

where we have again substituted cq (4.2) for the rotation-coefficient-matrix element, and suppressed the zero subscripts on the Euler angles $\theta$ and $\phi$. The preceding section shows that if the probe antenna is a reciprocal antenna, then eq (6.26) becomes (6.19), which is identical to

$$
b_{G}=\frac{a_{0}^{\prime \prime} \hat{\eta}_{0}^{\prime \prime} k^{2}}{2 \pi \eta} \sum_{s n m}(-)^{m} \frac{2 n+1}{n(n+1)} R_{s,-m, n} \sum_{\mu} \hat{R}_{s \mu n}^{\prime} e^{i m \phi} d_{m, \mu}^{n}(\theta) e^{i \mu \chi} .
$$

As a consequence of eq (6.27), we can make the definition

$$
\tilde{Q}_{s m n} \equiv \frac{a_{0}^{\prime \prime} \hat{\eta}_{0}^{\prime \prime} k^{2}}{2 \pi \eta}(-)^{m} \frac{2 n+1}{n(n+1)} R_{s,-m, n}
$$

and discover, upon substituting into eq (6.27), that the result is identical in the form with eq (6.25). Consequently, we see that the form of the starting point equation for a probecorrected spherical-scanning algorithm is the same for both the transmitting test-antenna and receiving test-antenna cases, provided a reciprocal probe is used. Note, however, that there is no restriction on the test antenna. Also, we are mostly interested in the calculation of the far-field pattern, so actual values of the modal-expansion coefficients need not even be computed.

Thus, we have shown that the algorithm to bc used for the transmitting test-antenna case is also valid for calculating the far-field receiving pattern of a receiving test antenna. The test antenna may be reciprocal or nonreciprocal; however, it is assumed that the probe is reciprocal.

\subsubsection{Relating Probe Receiving Coeflicients in the Doubly-Primed Coordinate System to Probe Transmitting Coefficients in the Singly-Primed Coordinate System; Rotation of a Singly- Primed Antenna into the Orientation of a Doubly-Primed Antenna}

As stated in section 5.1, the only distinction between the trcatment of the two antenna systems depicted in figure 5-1 is that the z-axis points out of the throat of one antenna and into the throat of the other antenna. The question to be answered here is, if we have obtained the modal-cxpansion coefficients for an antenna in one such orientation, what are the modalexpansion coefficients for that same antenna in the other orientation?

In figure 6-1, we show the gcometry under discussion, where the doubly-primed coordinate system is obtained from the singly-primed coordinate systcm (without translation) by a $180^{\circ}$ rotation about the $y^{\prime}$-axis. It is assumed that the modal-cxpansion cocfticients $Q^{\prime s m n}$ are known for the orientation of the antenna shown on the right in figure 6-1, and that we wish to 


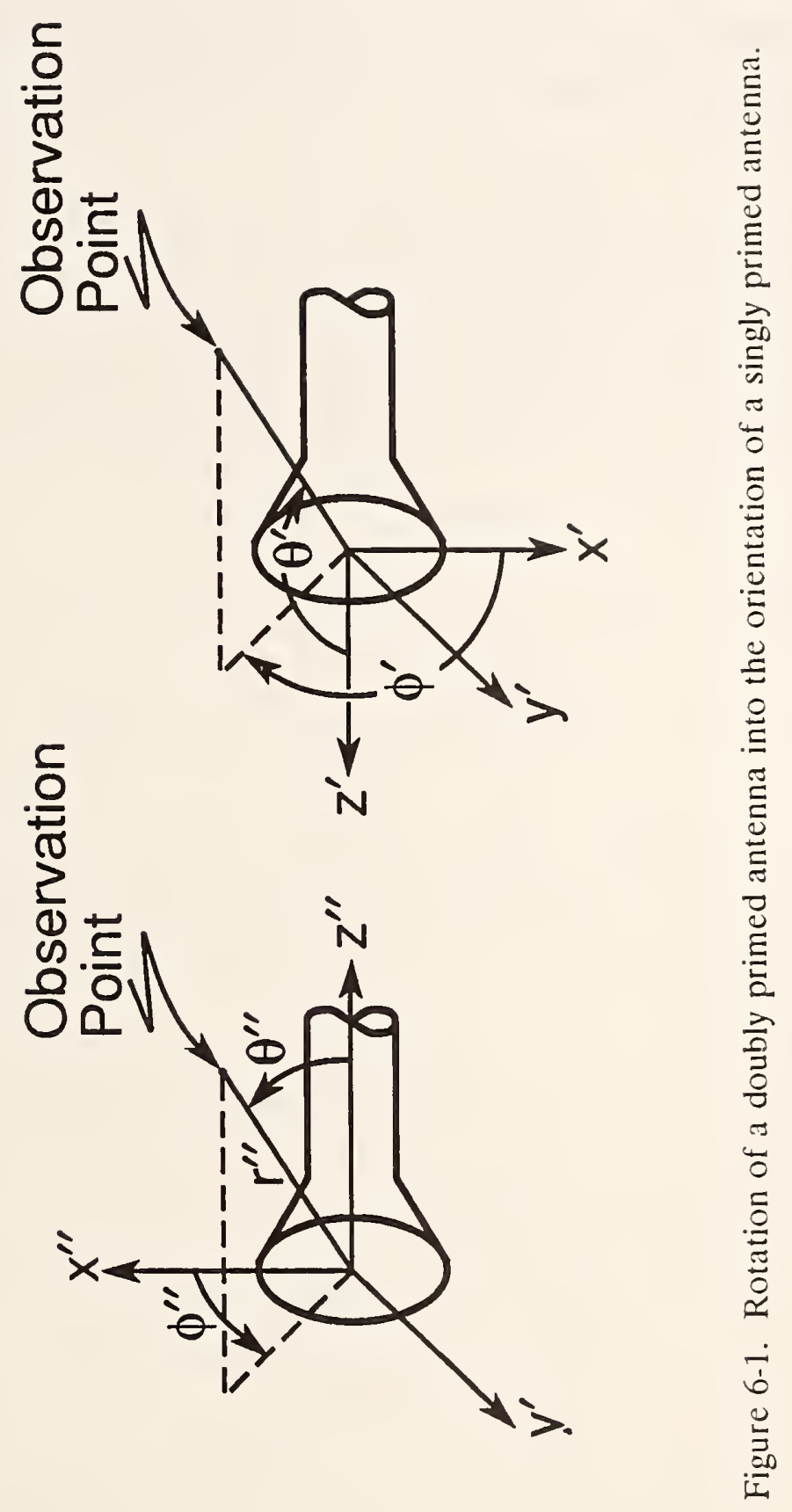


determine the modal-expansion coefficients $Q^{\prime \prime s m n}$ for the same antenna but oriented with respect to the doubly-primed coordinate system shown on the left in figure 6-1. Just the transmitting antenna case will be treated since the receiving antenna case readily follows.

Now from the expression (following eq (4.2)) for the coordinate system rotation coefficients we readily see that

$$
d_{m^{\prime} m}^{n}(\pi)=(-)^{n-m^{\prime}} \delta_{m^{\prime},-m}
$$

where $\delta_{\mathrm{ij}}$ is the Kronecker delta, which is zero for $\mathrm{i} \neq \mathrm{j}$ and is unity when $\mathrm{i}=\mathrm{j}$.

We next apply the rotation of coordinates transformation to the solution of the scalar wave equation (2.14). Consequently, by identifying eq (4.4) with the angular coordinate function in eq (2.14), we can apply the coordinate rotation transformation (4.1) to obtain

$$
\psi_{m n}^{(1)^{\prime \prime}} \underline{r}^{\prime \prime}=\sum_{m^{\prime}}\left(\psi_{m^{\prime} n}^{(1)^{\prime}} \underline{r}^{\prime}\right) D_{m^{\prime} m}^{n}(\alpha, \beta, \gamma),
$$

where we have explicitly noted that $\underline{r}^{\prime \prime}=\underline{r}^{\prime}$ is an invariant under coordinate system rotation. Then, noting that the curl operator is independent of the coordinate system, from eq (2.13) we see that applying the curl operator to both sides of the preceding result leads to

$$
\underline{M}_{m n}^{(1)^{\prime \prime}}=\sum_{m^{\prime}=-n}^{n} \underline{M}_{m^{\prime} n}^{(1)^{\prime}} D_{m^{\prime} m}^{n}(\alpha, \beta, \gamma) \text {. }
$$

Taking the curl of both sides of eq (6.30) then leads to the rotation of coordinates transformation of the $\underline{N}_{m n}^{(1)^{\prime \prime}}$ functions.

Returning to the problem of figure 6-1, we recall that we require a rotation of $180^{\circ}$ about the $y^{\prime}$ axis to transform coordinates from the singly-primed to the doubly-primed coordinate system. Consequently, with $\alpha=\gamma=0$ and $\beta=\pi$, the definition (4.2) and our prior result (6.29) substituted into (6.30) leads to the transformation expressions

$$
\begin{aligned}
& \underline{M}_{m n}^{(1)^{\prime \prime}}=(-)^{m+n} \underline{M}_{-m n}^{(1)^{\prime}}, \\
& \underline{N}_{m n}^{(1)^{\prime \prime}}=(-)^{m+n} \underline{N}_{-m n}^{(1)^{\prime}} .
\end{aligned}
$$

Since the radiated field is an invariant quantity, we obtain as a consequence of eq (6.31),

$$
\underline{E}=\sum_{s n m} Q^{\prime s m n} \underline{F}_{s m n}^{(1)^{\prime}}=\sum_{s n m}(-)^{m+n} Q^{\prime s,-m, n} \underline{F}_{s m n}^{(1)^{\prime \prime}} .
$$

Consequently, the modal-expansion coefficients transform according to the relationship

$$
Q^{/ / s m n}=(-)^{n+m} Q^{\prime s,-m, n}
$$


With the relationship eq (6.33), we can take an antenna whose modal-expansion coefficients are known, say with respect to the singly primed coordinate system of figure 5-2, place that antenna into the position of the doubly primed antenna system, and using eq (6.33) still know the modalexpansion coefficients in the new orientation. Note this relationship is independent of whether the antenna is reciprocal or not.

One very important application of eq (6.33) is that it allows us to obtain the receiving coefficients of a reciprocal antenna in the doubly primed coordinate system when we know, or have measured, that antenna's transmitting coefficients in the unprimed coordinate system. That is, for our spherical-scanning application we wish to obtain the receiving probe coefficients, in the doubly primed coordinate system, by measuring the probe's transmitting pattern relative to the unprimed coordinate system. Consequently, we combine eq (3.8), relating transmitting and receiving characteristics for reciprocal antennas, with eq (6.33), which transforms the modalexpansion coefficients in accord with a $180^{\circ}$ flip of the z-axis. There results

$$
\hat{R}_{\sigma \mu \nu}^{\prime \prime}=\frac{\pi \eta}{\hat{\eta}_{0} k^{2}}(-)^{\nu} \frac{v(v+1)}{\nu+1 / 2} T^{\sigma \mu \nu},
$$

whcre $T^{\sigma \mu \nu}$ represents the transmitting coefficients of a rcciprocal antenna oriented in a coordinate system whose z-axis points away from the antenna, while $\hat{R}_{\sigma \mu \nu}^{\prime \prime}$ represents the receiving characteristics of the same antenna but oriented in a coordinate system whose z-axis points into the antenna.

\subsection{On the Validity of Interchanging the Order of Summation of $\mathbf{n} \leftrightarrow v$ Infinite Double Series}

An integral part of the development presented in Chapter 5 involved the order of summation interchange (eq (5.9)). There, the justification for interchanging the order of summation of the infinite $\mathrm{n}$ and $v$ sums was deferred to this chapter. Here, using Pringsheim's theorem, we shall show that this interchange is justified. Pringsheim's theory states [6] that if the sum of rows $\sum_{n=1}^{\infty} a_{v n}$ each converge, the sum of columns $\sum_{v=1}^{\infty} a_{v n}$ each converge, and if the doublc series is convergent to a sum, s, then we have

$$
s=\sum_{v=1}^{\infty}\left(\sum_{n=1}^{\infty} a_{v n}\right)=\sum_{n=1}^{\infty}\left(\sum_{v=1}^{\infty} a_{v n}\right) .
$$

Thus, we have to show that these three prerequisites to eq (6.35) arc satisficd for each application of the summation interchangc (5.9). 
In order to carry this out, we need to introduce additional coordinate system notation. Referring to figure 5-2, we see that the observation point is described by two sets of coordinates referenced to two distinct coordinate systems. We can characterize this dual representation by observing that the observation point is at location $r^{\prime}$, relative to the singly primed coordinate system, and at location $r^{\prime \prime}$, relative to the doubly primed coordinate system. We also need to describe the location of a source point with respect to both coordinate systems. This source point (not shown) could be located within the confines of the singly primed antenna system or the doubly primed antenna system. However, the immediate presentation will just consider source points located within the confines of the singly primed antenna system. We adopt the convention that such source-point locations are at $\underline{r}^{\prime \prime \prime}$, relative to the singly primed coordinate system, and at $\underline{I}^{I V}$, relative to the doubly primed coordinate system. We will also want to characterize the situation depicted in figure 5-1. There, the observation point's location is described with respect to the unprimed coordinate-system and the doubly primed coordinate system as $r$ and $\underline{r}^{\prime \prime}$, respectively. We adopt the convention with figure 5-1 that the source point's location is at $\underline{r}^{\prime \prime \prime}$ with respect to the unprimed coordinate system and at $\underline{r}^{I V}$ with respect to the doubly primed coordinate system. Since here we do not consider the singly primed coordinate system and the unprimed coordinate system simultaneously, no confusion over this dual use of $\underline{r}^{\prime \prime \prime}$ and $\underline{r}^{I V}$ should result. Continuing our earlier notation, primes (or $I V$ ) are appended to the vector spherical-wave functions to designate the applicable coordinate system.

Referring now to figure 5-2 we consider the situation where we are interested in excitation currents on the singly primed antenna. The electric field at $\underline{r}^{\prime}$ can be expressed, in a manner similar to eq (2.16), as

$$
\underline{E}\left(\underline{r}^{\prime}\right)=i \omega \mu \iiint \underline{\underline{G}}\left(\underline{r}^{\prime} \mid \underline{r}^{\prime \prime \prime}\right) \cdot \underline{J}^{\prime}\left(\underline{r}^{\prime \prime \prime}\right) d v^{\prime \prime \prime},
$$

where $\underline{J}^{\prime}\left(\underline{r}^{\prime \prime \prime}\right)$ is the current distribution on the singly primed antenna, and where (see eq (2.25))

$$
\underline{\underline{G}}\left(\underline{r}^{\prime} \mid \underline{r}^{\prime \prime \prime}\right)=\frac{i k}{4 \pi} \sum_{n=1}^{\infty} \sum_{m=-n}^{n} \frac{2 n+1}{n(n+1)}\left[\underline{M}_{m n}^{(1)^{\prime}} \underline{M}_{m n}^{\prime \prime \prime}+\underline{N}_{m n}^{(1)^{\prime}} \underline{N}_{m n}^{\prime \prime \prime} *\right], r^{\prime \prime \prime}<r^{\prime} .
$$

Here, we have a uniformly convergent power series for $\frac{r^{\prime \prime \prime}}{r^{\prime}}<1$. We also have [19] the uniformly convergent series eq (4.23) when $\frac{r^{\prime \prime}}{d}<1$. Upon substituting eq (4.23) into eq (6.37) we obtain

$$
\begin{aligned}
\underline{\underline{G}}\left(\underline{\underline{r}}^{\prime} \mid \underline{r}^{\prime \prime \prime}\right)=\frac{i k}{4 \pi} \sum_{n=1}^{\infty} \sum_{m=-n}^{n} \frac{2 n+1}{n(n+1)} & \left\{\sum_{v=(1,|m|)}^{\infty}\left(\underline{M}_{m v}^{\prime \prime} A_{m v}^{m n}+\underline{N}_{m v}^{\prime \prime} B_{m v}^{m n}\right) \underline{M}_{m n}^{\prime \prime \prime} *\right. \\
& +\sum_{v=(1,|m|)}^{\infty}\left(\underline{N}_{m v}^{\prime \prime} A_{m v}^{m n}+\underline{M}_{m v}^{\prime \prime} B_{m v}^{m n}\right) \underline{N}_{m n}^{\prime \prime \prime} *
\end{aligned} .
$$


Substituting eq (6.11) into eq (6.38) produces

$$
\begin{aligned}
\underline{G}\left(\underline{r}^{\prime} \mid \underline{r}^{\prime \prime \prime}\right)= & \frac{i k}{4 \pi} \sum_{n=1}^{\infty} \sum_{m=-n}^{n} \sum_{v=(1,|m|)}^{\infty} \frac{2 v+1}{v(v+1)}\left\{\underline{M}_{m v}^{\prime \prime}\left(a_{m n}^{m v} \underline{M}_{m n}^{\prime \prime \prime}-^{*} b_{m n}^{m v} \underline{N}_{m n}^{\prime \prime \prime}\right)\right. \\
& \left.+\underline{N}_{m v}^{\prime \prime}\left(a_{m n}^{m v} \underline{N}_{m n}^{\prime \prime \prime}-b_{m n}^{m v} \underline{M}_{m n}^{\prime \prime \prime}\right)\right\} \\
= & \frac{i k}{4 \pi} \sum_{n=1}^{\infty} \sum_{m=-n}^{n} \sum_{v=(1,|m|)}^{\infty} \frac{2 v+1}{v(v+1)}\left\{\underline{M}_{m v}^{\prime \prime *}\left(a_{m n}^{m v} \underline{M}_{m n}^{\prime \prime \prime}+b_{m n}^{m v} \underline{N}_{m n}^{\prime \prime \prime}\right)\right. \\
& \left.+\underline{N}_{m v}^{\prime \prime *}\left(a_{m n}^{m v} \underline{N}_{m n}^{\prime \prime \prime}+b_{m n}^{m v} \underline{M}_{m n}^{\prime \prime \prime}\right)\right\},
\end{aligned}
$$

where we have reversed the summation order on the $m$ index and applied eqs (2.24) and (6.12) to finally obtain eq (6.39).

The summation over $n$ of the terms in eq (6.39) results in the two sums

$$
\begin{gathered}
\sum_{n=(1,|m|)}^{\infty}\left(a_{m n}^{m v} \underline{M}_{m n}^{\prime \prime \prime}+b_{m n}^{m v} \underline{N}_{m n}^{\prime \prime \prime}\right)=\underline{M}_{m v}^{(1)^{I V}} \\
\sum_{n=(1,|m|)}^{\infty}\left(a_{m n}^{m v} \underline{N}_{m n}^{\prime \prime \prime}+b_{m n}^{m v} \underline{M}_{m n}^{\prime \prime \prime}\right)=\underline{N}_{m v}^{(1)^{I v}},
\end{gathered}
$$

where we have used eq (6.3) to identify the result of the summation over $n$, taking note of the fact that the terms in the summation are each equal to zero for $n<m$. The range of $\underline{r}^{\prime \prime \prime}$ is $r^{\prime \prime \prime} \leq \rho<d$, where $\rho$ is the maximum extent of nontrivial $\underline{J}^{\prime}\left(\underline{r}^{\prime \prime \prime}\right)$ values. Consequently, since eqs (6.39), (4.23), and (6.40) all converge, the conditions for applying Pringsheim's theorem are all satisfied. Moreover, $d-\rho<r^{I V}<d+\rho$. Furthermore, we must have $r^{\prime \prime}<d-\rho$ since the circumscribing sphere about the doubly primed coordinate system origin is so defined that it does not contain external sources. Therefore, $r^{\prime \prime}<r^{I V}$. Now using eq (5.9) we apply Pringsheim's theorem to eq (6.39) to obtain

$$
\begin{aligned}
\underline{\underline{G}}\left(\underline{r}^{\prime} \mid \underline{r}^{\prime \prime \prime}\right)=\frac{i k}{4 \pi} \sum_{v=1}^{\infty} \sum_{m=-v}^{v} \frac{2 v+1}{v(v+1)}\left\{\underline{M}_{m v}^{\prime *} \sum_{n=(1,|m|)}^{\infty}\left(a_{m n}^{m v} \underline{M}_{m n}^{\prime \prime \prime}+b_{m n}^{m v} \underline{N}_{m n}^{\prime \prime \prime}\right)\right. \\
\left.+\underline{N}_{m v}^{\prime \prime} \sum_{n=(1,|m|)}^{\infty}\left(a_{m n}^{m v} \underline{N}_{m n}^{\prime \prime \prime}+b_{m n}^{m v} \underline{M}_{m n}^{\prime \prime \prime}\right)\right\} .
\end{aligned}
$$

Consequently, using eq (6.40) with this result produces the expression

$$
\underline{\underline{G}}\left(\underline{r}^{\prime} \mid \underline{r}^{\prime \prime \prime}\right)=\frac{i k}{4 \pi} \sum_{v=1}^{\infty} \sum_{m=-v}^{v} \frac{2 v+1}{v(v+1)}\left\{\underline{M}_{m v}^{\prime *} \underline{M}_{m v}^{(1)^{I V}}+\underline{N}_{m v}^{\prime \prime *} \underline{N}_{m v}^{(1)^{N}}\right\}, \quad r^{\prime \prime}<r^{I V} .
$$

The right side of eq (6.42) is seen to be equivalent to the right side of eq (2.25), except that radii in eq (6.42) are referenced to the doubly primed coordinate system origin while radii in eq (2.25) 
are referenced to the unprimed system's origin of coordinates. Thus, with eq (6.37) we started out with a Green's function appropriate for characterizing electric fields in a region exterior to the source region, and wound up with eq (6.42) which is appropriate for characterizing electric fields in an interior region with respect to external sources. Consequently, an alternate representation for the electric field given by eq $(6.36)$ is

$$
\underline{E}\left(\underline{r}^{\prime \prime}\right)=i \omega \mu \iiint \underline{\underline{G}}\left(\underline{r}^{\prime \prime} \mid \underline{r}^{I V}\right) \cdot \underline{J}^{\prime}\left(\underline{r}^{I V}\right) d v^{I V},
$$

where $\underline{\underline{G}}\left(\underline{r}^{\prime \prime} \mid \underline{r}^{l V}\right)$ is given by eq (6.42); that is

$$
\underline{\underline{G}}\left(\underline{r}^{\prime \prime} \mid \underline{r}^{I V}\right)=\underline{\underline{G}}\left(\underline{r}^{\prime} \mid \underline{r}^{\prime \prime \prime}\right) \text {. }
$$

Thus, we have established an identity between two Green's function representations. It is noteworthy to add that the derivation can readily be reversed; that is, by starting with eq (6.43) where $\underline{\underline{G}}\left(\underline{r}^{\prime \prime} \mid \varepsilon^{l V}\right)$ is given by eq (6.42) and using Pringsheim's theorem to justify the resulting $\mathrm{n} \leftrightarrow v$ summation interchange, we reobtain eqs (6.36) and (6.37).

Let us now combine eqs (6.36) and (6.37) and compare that result with (5.7). Since eq (6.37) is uniformly convergent, we have

$$
\begin{aligned}
\underline{E}\left(\underline{r}^{\prime}\right)=- & \frac{\omega \mu k}{4 \pi} \sum_{n=1}^{\infty} \sum_{m=-n}^{n} \frac{2 n+1}{n(n+1)}\left\{\underline{M}_{m n}^{(1)^{\prime}}\left[\int_{0}^{\rho}\left(r^{\prime \prime \prime}\right)^{2} d r^{\prime \prime \prime} \iint_{\Omega} d \Omega^{\prime \prime \prime} \underline{M}_{m n}^{\prime \prime \prime *} \cdot \underline{J}^{\prime}\left(\underline{\underline{r}}^{\prime \prime \prime}\right)\right]\right. \\
& \left.+\underline{\underline{N}}_{m n}^{(1)^{\prime}}\left[\int_{0}^{\rho}\left(r^{\prime \prime \prime}\right)^{2} d r^{\prime \prime \prime} \iint_{\Omega} d \Omega^{\prime \prime \prime} \underline{\underline{N}}_{m n}^{\prime \prime \prime *} \cdot \underline{J}^{\prime}\left(\underline{r}^{\prime \prime \prime}\right)\right]\right\} \\
= & \sum_{n=1}^{\infty} \sum_{\mu=-n}^{n}\left\{Q^{\prime 1 \mu n} \underline{M}_{\mu n}^{(1)^{\prime}}+Q^{\prime 2 \mu n} \underline{N}_{\mu n}^{(1)^{\prime}}\right\} .
\end{aligned}
$$

Equating like vector coefficients, we obtain

$$
\begin{aligned}
& Q^{\prime 1 \mu n}=-\frac{\omega \mu k}{4 \pi} \frac{2 n+1}{n(n+1)} \int_{0}^{\rho}\left(r^{\prime \prime \prime}\right)^{2} d r^{\prime \prime \prime} \iint_{\Omega} d \Omega^{\prime \prime \prime} \underline{M}_{\mu n}^{\prime \prime \prime *} \cdot \underline{J}^{\prime}\left(\underline{r}^{\prime \prime \prime}\right) \\
& Q^{\prime 2 \mu n}=-\frac{\omega \mu k}{4 \pi} \frac{2 n+1}{n(n+1)} \int_{0}^{\rho}\left(r^{\prime \prime \prime}\right)^{2} d r^{\prime \prime \prime} \iint_{\Omega} d \Omega^{\prime \prime \prime} \underline{N}_{\mu n}^{\prime \prime *} \cdot \underline{J}^{\prime}\left(\underline{r}^{\prime \prime \prime}\right),
\end{aligned}
$$

where the integration over $\Omega$ implies integration over the angular spherical coordinates.

Similarly, combining eqs (6.42) and (6.43) and comparing that result with eq (5.10) produces

$$
\begin{aligned}
& P_{1 \mu \nu}^{\prime \prime}=-\frac{\omega \mu k}{4 \pi} \frac{2 v+1}{v(v+1)}(-)^{\mu} \int_{0}^{\rho}\left(r^{\prime \prime \prime}\right)^{2} d r^{\prime \prime \prime} \iint_{\Omega} d \Omega^{\prime \prime \prime} \underline{M}_{-\mu, \nu}^{(1)} \cdot \underline{J}^{I V}\left(\underline{r}^{\prime \prime \prime}\right) \\
& P_{2 \mu \nu}^{\prime \prime}=-\frac{\omega \mu k}{4 \pi} \frac{2 v+1}{v(v+1)}(-)^{\mu} \int_{0}^{\rho}\left(r^{\prime \prime \prime}\right)^{2} d r^{\prime \prime \prime} \iint_{\Omega} d \Omega^{\prime \prime \prime} \underline{N}_{-\mu, \nu}^{(1)^{I V}} \cdot \underline{J}^{\prime}\left(\underline{r}^{\prime \prime \prime}\right) .
\end{aligned}
$$


Thus, we have established that $Q^{\prime s \mu n}$ behaves like $\underline{F}_{s \mu n}^{\prime \prime *}$, while $P_{o \mu v}^{\prime \prime}$ behaves like $\underline{F}_{\sigma,-\mu, v}^{(1)^{N v}}$, referring to the notation of eq (2.45). In eq (6.46), $\underline{r}^{I V}$ is an implicit function of $\underline{r}^{\prime \prime \prime}$.

We are now in a position to consider the summation interchange in eq (5.8). However, it would be more interesting to be a little more general and show the complete transformation of the electric-field vector from the unprimed coordinate system to the doubly primed coordinate system, rather than just limiting our discussion to the derivation of eq (5.11) starting from eq (5.7). Consequently, using the alternate notation of eq (2.46), we can express the electric field, radiated by the antenna on the left in figure 5-1, as

$$
\underline{E}=\sum_{s n m} Q^{s m n} \underline{F}_{s m n}^{(1)}
$$

We wish to substitute the vector spherical-wave functions, rotated into the singly primed coordinate system, for the $\underline{F}_{s m n}^{(1)}$ in eq (6.47). To do this, in view of eq (6.30), we need to take the inverse of the transformation (4.1). Accordingly, from eqs (4.8) and (4.9), with $\alpha, \beta, \gamma$ identificd as $\phi_{0}, \theta_{0}, \chi$ (sce eq (5.5)), we have,

$$
\underline{F}_{s m n}^{(1)}=\sum_{\mu} \underline{F}_{s \mu n}^{(1)^{\prime}} D_{m \mu}^{n *}\left(\phi_{0}, \theta_{0}, \chi\right) .
$$

Next we substitute eq (5.13) into eq (6.48) and that result into eq (6.47) to obtain

$$
\underline{E}=\sum_{s=1}^{2} \sum_{n=1}^{\infty} \sum_{m=-n}^{n} Q^{s m n} \sum_{\mu=-n}^{n}\left[\sum_{\sigma=1}^{2} \sum_{\nu=(1,\{\mu \mid)}^{\infty} C_{\sigma \mu \nu}^{s n} \underline{F}_{\sigma \mu \nu}^{\prime \prime}\right] D_{m \mu}^{n *}\left(\phi_{0}, \theta_{0}, \chi\right)
$$

In order to apply Pringsheim's theorem to invert the order of summation of the $\mathrm{n}$ and $v$ series in cq (6.49), we necd only show that the sum over terms in $n$ in eq (6.49) converges. We do this by using $\mathrm{cq}(5.5)$ and then substituting eq (6.45) for $Q^{/ \text {sun }}$ into eq (6.49); then, carrying out an obviously permissible interchange in the order of summation and integration in that result, we find that eq (6.49) becomes expressible as the integral of the dot product of $\underline{\underline{J}}^{\prime}\left(\underline{r}^{\prime \prime}\right)$ with eq (6.38). Repeating the process which led from eq (6.38) to eq (6.41), we can bring the integration back inside the summations and use eqs (6.11) and (6.45) to reconvert the integration over $\underline{r}^{\prime \prime \prime}$ back to $Q^{\prime s \mu n}$. Then eq (5.5) completes eq (6.49)'s summation interchange, producing

$$
\underline{E}=\sum_{\sigma=1}^{2} \sum_{\nu=1}^{\infty} \sum_{\mu=-\nu}^{\nu}\left[\sum_{s=1}^{2} \sum_{n=(1,|\mu|)}^{\infty} \sum_{m=-n}^{n} C_{\sigma \mu \nu}^{s n} Q^{s m n} D_{m \mu}^{n *}\left(\phi_{0}, \theta_{0}, \chi\right)\right] \underline{F}_{\sigma \mu \nu}^{\prime \prime} .
$$

Now, eqs (6.50) and (5.10) are equivalent expressions for the electric field. Consequently, we can equate like coefficients of the vector spherical-wave functions to obtain

$$
P_{\sigma \mu \nu}^{\prime \prime}=\sum_{s n m} C_{\sigma \mu \nu}^{s n} Q^{s m n} D_{m \mu}^{n *}\left(\phi_{0}, \theta_{0}, \chi\right)
$$


Substituting eq (6.51) into the equivalent generator-source wave expression (5.1) produces

$$
b_{G}^{\prime \prime}=\sum_{\sigma=1}^{2} \sum_{\nu=1}^{\infty} \sum_{\mu=-\nu}^{\nu} \hat{R}_{\alpha \mu \nu}^{\prime \prime}\left[\sum_{s=1}^{2} \sum_{n=(1,|\mu|)}^{\infty} \sum_{m=-n}^{n} C_{\sigma \mu \nu}^{s n} Q^{s m n} D_{m \mu}^{n *}\left(\phi_{0}, \theta_{0}, \chi\right)\right] \text {. }
$$

Without loss of generality, we assume that the doubly primed antenna is reciprocal, or at least adjoint reciprocal [26, Chapt. II, Appendix A]. Furthermore, we make the distinction that the vector $\underline{r}^{I \bar{V}}$ is a radial vector from the doubly primed coordinate-system origin to (induced) current sources on the doubly primed antenna, while $\underline{r}^{I V}$ is a radial vector from the same coordinate-system origin to current sources on the singly primed antenna. Then, by eq (3.8), $\hat{R}_{\sigma \mu \nu}^{\prime \prime}$ is proportional to $\hat{T}^{\prime \prime \sigma,-\mu, \nu}$, which in turn is proportional to $\hat{Q}^{\prime \prime \sigma,-\mu, \nu}$ by eq (5.28). Consequently, in analogy with eq (6.45), we establish that $\hat{R}_{\sigma \mu \nu}^{\prime \prime}$ behaves like $\underline{F}_{\sigma \mu \nu}^{I \bar{V}}$, where $\underline{r}^{\bar{V}}$ is integrated over the spherical region $0 \leq r^{I V} \leq \bar{\rho}$ with $\bar{\rho}$ designating the radius of a minimum circumscribing sphere enclosing the doubly primed antenna system. Then, in view of eq (6.46), which establishes that $P_{\sigma \mu \nu}^{\prime \prime}$ behaves like $\underline{F}_{\sigma,-\mu, \nu}^{(1)^{I V}}$, where $d-\rho<r^{I V}<d+\rho$, we establish that the sum (5.1) is a power series in $r^{I V} / r^{I V}<1$; thus, it converges uniformly in the region of interest. We have shown that eq (6.51) is convergent, so in order to apply Pringsheim's theorem to eq (6.52) we just need to show that the sum over $v$ alone in eq (6.52) is convergent. However, this is just the sum of terms $\hat{R}_{\sigma \mu \nu}^{\prime \prime} C_{\sigma \mu \nu}^{s n}$, and we have just argued that these terms are each proportional to an integral, over $\underline{r}^{\bar{I}}$, of $C_{\sigma \mu \nu}^{s n} \underline{F}_{\sigma \mu \nu}^{I \bar{V}} \cdot \underline{J}^{\prime \prime}\left(\underline{r}^{I \bar{V}}\right)$. Consequently, in view of eq (5.13), the sum over $v$ in eq (6.52) must converge uniformly provided $r^{\bar{V}}<d$; hence, Pringsheim's theorem applies to eq (6.52). Using eq (5.9) to effect the summation interchange, we obtain the result (6.1).

In the preceding paragraphs, we have presented our arguments justifying the summation interchange (5.9) as applied to eqs (5.4) and (5.8). The justification for the summation interchange in eq (5.14) is similar to the argument given for arriving at eq (6.50). Therefore, we have only the summation interchange in going from eq (5.28b) to (5.29b) still to consider.

In eq (5.28b), we can set $a_{0}^{\prime \prime}=0$ and $P_{\sigma \mu \nu}^{\prime \prime}=\delta_{\sigma \sigma^{\prime}} \delta_{\mu \mu^{\prime}} \delta_{\nu \nu^{\prime}}$ to show that $\hat{S}_{\sigma \mu \nu}^{\prime \prime s m n}$ is equal to a particular $\hat{Q}^{\prime \prime s m n}$, which by eq (6.45) is proportional to the integral over $\underline{r}^{I \bar{V}}$ of $\underline{F}_{\sigma \mu \nu}^{I^{*} *}$, where $0 \leq r^{\overline{I V}}<\bar{\rho}$. Next, using the reciprocity relation (3.9), we see similarly that 
$\hat{S}_{\sigma \mu \nu}^{/ / s m n}$ is proportional to some integral over $\underline{r}^{\check{I V}}$ of $\underline{F}_{\sigma \mu \nu}^{\check{I V}}$, where $0 \leq r^{\check{I V}}<\bar{\rho}$. Thus, we have argued that

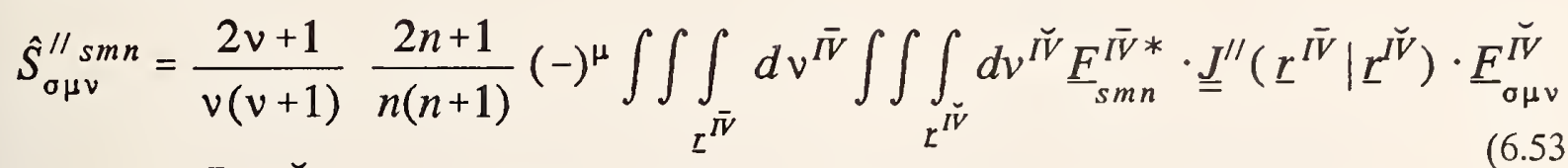

where $\underline{\underline{J}}^{\prime \prime}\left(\underline{r}^{I \bar{V}} \mid \underline{r}^{\check{I V}}\right)$ is a dyadic independent of the scattering-matrix element's indexes.

Now, from eq (6.46), we see that $P_{\sigma \mu \nu}^{\prime \prime}$ is proportional to an integral over $\underline{r}^{/ / /}$of $\underline{F}_{\sigma,-\mu, \nu}^{(1)}$, where $r^{I V}\left(\underline{r}^{I / /}\right)>d-\rho$. Consequently, the sum over $v$ of $\hat{S}_{\sigma \mu \nu}^{\prime / s m n} P_{\sigma \mu \nu}^{\prime \prime}$ is a uniformly convergent power series of terms of the order of $r^{I V} / r^{I V}<1$. The argument leading to eq (6.50) has established that eq (6.51), or equivalently eq (5.2), is uniformly convergent. We next substitute eq (5.2) into eq (5.28b) to obtain

$$
\hat{Q}^{\prime \prime p m r}=\hat{T}^{\prime \prime p m r} a_{0}^{\prime \prime}+\sum_{\kappa \gamma \mu} \hat{S}_{\kappa \mu \gamma}^{\prime \prime p m r} \sum_{\sigma \nu} C_{\kappa \mu \gamma}^{\sigma \nu} Q^{\prime \sigma \mu \nu} .
$$

Since eq (5.13) is uniformly convergent, it follows from eq (6.53) that the infinite sum over $\gamma$ in eq (6.54) is also uniformly convergent. Therefore, Pringsheim's theorem applies, so we can use eq (5.9) to effect the $\gamma \leftrightarrow v$ summation interchange, obtaining

$$
\hat{Q}^{\prime \prime p m r}=\hat{T}^{\prime \prime p m r} a_{0}^{\prime \prime}+\sum_{\sigma \nu \mu}\left(\sum_{\kappa \gamma} C_{\kappa \mu \gamma}^{\sigma \nu} \hat{S}_{\kappa \mu \gamma}^{\prime \prime p m r}\right) Q^{\prime \sigma \mu \nu} .
$$

Next, we note that establishing the summation interchange in eq (5.14), which we see is similar to establishing the summation interchange in eq (5.8), guarantees the uniform convergence of eq (5.18). We substitute eq (6.55) into eq (5.18) to obtain

$$
\hat{P}_{s m n}^{\prime}=\sum_{p r} \alpha_{s m n}^{p r} \hat{T}^{\prime \prime p m r} a_{0}^{\prime \prime}+\sum_{p r} \alpha_{s m n}^{p r} \sum_{\sigma \nu \mu}\left(\sum_{\kappa \gamma} C_{\kappa \mu \gamma}^{\sigma \nu} \hat{S}_{\kappa \mu \gamma}^{\prime \prime p m r}\right) Q^{\prime \sigma \mu \nu} .
$$

From eq (6.53) and the definition of $\alpha_{s m n}^{p r}$ (see eqs (5.17) and (6.4)), it is apparent that the terms $\alpha_{s m n}^{p r} \hat{S}_{\kappa \mu \gamma}^{\prime / p m r}$ asymptotically vary with respect to the index r like $\left(r^{\bar{N}} / d\right)^{r}<(\bar{\rho} / d)^{r}$; therefore the sum $\sum_{p r} \alpha_{s m n}^{p r} \hat{S}_{\kappa \mu \gamma}^{\prime \prime p m r}$ converges uniformly so we can interchange the $\mathrm{r}$ and $v$ summations in eq (6.56) to obtain

$$
\hat{P}_{s m n}^{\prime}=\sum_{p r} \alpha_{s m n}^{p r} \hat{T}^{\prime \prime p m r} a_{0}^{\prime \prime}+\sum_{\sigma \nu \mu}\left(\sum_{p r} \alpha_{s m n}^{p r} \sum_{\kappa \gamma} C_{\kappa \mu \gamma}^{\sigma \nu} \hat{S}_{\kappa \mu \gamma}^{\prime \prime p m r}\right) Q^{\prime \sigma \mu \nu} .
$$

Thus, comparing eq (6.57) with the defining eq (5.29b) yields the previously given expressions $(5.30 \mathrm{c})$ and $(5.30 \mathrm{~d})$. 
In conclusion then, we have proven in this section that our applications of the summation formula (5.9) were justified, and we have established a translation-of-coordinates-transformation identity for the dyadic Green's function.

\subsection{Conclusions}

In this chapter we have established the equalities (6.11) and (6.12) between contragradient vector-wave function translation coefficients, which has led to the development of a simple reciprocity relationship (eq (6.22)) between signals received by reciprocal antennas when multiple reflections are negligible. Of significant import for this chapter is the proof (section 6.4.1) that just a single algorithm need be developed for spherical near-field to far-field transformations (spherical scanning) for calculating either the transmitting or receiving patterns of nonreciprocal antennas, provided that the probe is reciprocal.

We have also established a reciprocity relation (eq (6.34)) between spherical transmitting and receiving coefficients taking into account the $180^{\circ}$ flip in the z-coordinate axis when going from the test antenna (unprimed) coordinate system to the probe antenna (doubly primed) coordinate system. This relationship permits us to obtain probe receiving coefficients in the probe's coordinate system from spherically scanned measurements of the probe's transmitting pattern. We will use these probe receiving coefficients in Chapter VIII to correct for the probe's effects on spherically scanned data.

We concluded this chapter with a proof that the interchange in eq (5.9) of infinite double series was justified, thereby validating the infinite double series interchanges in Chapter V. In doing this, we established a coordinate-system translation theorem (eq (6.44)) for the dyadic Green's function.

In the next chapter, we develop equalities between the series representation of the electric field and the coupling equations when one antenna is an elementary dipole. 


\section{CHAPTER VII}

\section{REDUCTION OF THE TWO-ANTENNA COUPLING EQUATION TO THE MODAL-EXPANSION EQUATION WHEN ONE ANTENNA IS AN ELEMENTARY DIPOLE}

\subsection{Introduction}

An important case study of two coupled antennas occurs when one antenna is an elementary electric dipole. Of particular importance is the fact that the open-circuit voltage at the terminals of a receiving elementary electric dipole is proportional to the incident electric field at that point. Consequently, the coupling eq (5.6) (or (6.1)) should transform into the radiating components of eq (2.42) for the transverse electric field when the doubly primed antenna is a receiving elementary electric dipole. Similarly, when the doubly primed antenna is a transmitting elementary electric dipole, the coupling eq (5.22) (or (6.2)) should reduce to results compatible with the signal received from a dipole source. The incident field due to such a source was obtained in Chapter II (eq (2.27)), while the received signal is given by eq (3.2). In this chapter, then, we shall establish equalities between the two-port coupling equations and the modalexpansion representation of the electric field for the case when one antenna is an ideal dipole.

\subsection{Modal Expansion Coefficients for an Elementary Dipole Current Source}

The starting point for the current analysis is to determine the modal-expansion coefficients for an elementary electric dipole in a coordinate system centered on the dipole antenna. We shall examine the case of a dipole oriented parallel to the $\mathrm{x}$-axis of its coordinate system, as well as the case of a dipole oriented parallel to the y-axis. Following the lead of a similar analysis by Tai [51], a dyadic Greens function formulation will be used to obtain the electric-field vector for such antennas, and from this result the modal-expansion coefficients can readily be obtained.

We consider a vector current-density function due to a radiating electric-dipole source oriented parallel to the $\mathrm{x}^{\prime \prime}$-axis (here, as in section 2.3 of Chapter II, double primes denote dipole-source coordinates rather than an alternate antenna-system's coordinates). Such a current element, located at $\mathrm{r}^{\prime \prime}=\mathrm{r}_{0}, \theta^{\prime \prime}=0$, and $\phi^{\prime \prime}=0$, may be expressed as 


$$
\underline{J}\left(\underline{r}^{\prime \prime}\right)=I d \ell \frac{\delta\left(r^{\prime \prime}-r_{0}\right) \delta\left(\theta^{\prime \prime}-0\right) \delta\left(\phi^{\prime \prime}-0\right)}{r_{0}^{2} \sin \theta^{\prime \prime}} \underline{a}_{x^{\prime \prime}},
$$

where Id $\ell$ is the dipole moment, and $\underline{\mathrm{a}}_{\mathrm{x}^{\prime}}$ is a unit vector parallel to the $\mathrm{x}^{\prime \prime}$-axis. $\underline{\mathrm{a}}_{\mathrm{x}^{\prime \prime}}$ can be expressed in terms of unit spherical basis vectors as

$$
\underline{a}_{x^{\prime \prime}}=\underline{a}_{r^{\prime \prime}} \cos \phi^{\prime \prime} \sin \theta^{\prime \prime}+\underline{a}_{\theta^{\prime \prime}} \cos \phi^{\prime \prime} \cos \theta^{\prime \prime}-\underline{a}_{\phi^{\prime \prime}} \sin \phi^{\prime \prime} .
$$

The electric field in the region exterior to the dipole source is given by eq (2.16) as

$$
\underline{E}(\underline{r})=i \omega \mu \iiint \underline{G}\left(\underline{r} \mid \underline{r}^{\prime \prime}\right) \cdot \underline{J}\left(\underline{r}^{\prime \prime}\right) d v^{\prime \prime},
$$

where the dyadic Greens function is obtained from a slight variation of eq (2.25) as

$$
\underline{\underline{G}}\left(\underline{r} \mid \underline{r}^{\prime \prime}\right)=\frac{i k}{4 \pi} \sum_{n=1}^{\infty} \sum_{m=-n}^{n} \frac{2 n+1}{n(n+1)}\left[\underline{M}_{m n}^{(1)} \underline{M}_{m n}^{\prime \prime *}+\underline{N}_{m n}^{(1)} \underline{N}_{m n}^{\prime \prime *}\right], \quad r>r^{\prime \prime} \text {. }
$$

Next, we need to consider the behavior of the associated Legendre function as the angle $\theta$ approaches zero. From ref. [3] we have the formula,

$$
P_{n}^{m}(x)=(-2)^{-m} \frac{(n+m) !}{m !(n-m) !}\left(1-x^{2}\right)^{\frac{m}{2}}{ }_{2} F_{1}\left\{1+m+v, m-v ; 1+m ; \frac{1-x}{2}\right\},
$$

which uses the hypergeometric function. As $\mathrm{x}$ approaches one, the hypergeometric function in eq (7.5) also approaches one. Consequently, we have

$$
\left.\frac{m P_{n}^{m}(\cos \theta)}{\sin \theta}\right]_{\theta=0}=-\frac{1}{2} \delta_{m,-1}-\frac{n(n+1)}{2} \delta_{m, 1},
$$

where once again we have introduced the Kronecker delta. Also, from ref. [3] it follows that

$$
\left.\frac{\partial P_{n}^{m}(\cos \theta)}{\partial \theta}\right]_{\theta=0}=\frac{1}{2} \delta_{m,-1}-\frac{n(n+1)}{2} \delta_{m, 1} .
$$

As a consequence of eqs (7.6), we have

and

$$
\left.\underline{M}_{m n}^{\prime \prime *} \cdot \underline{a}_{x^{\prime \prime}}\right]_{\theta^{\prime \prime}=0}=i \frac{\sqrt{n(n+1)}}{2} j_{n}\left(k r^{\prime \prime}\right)\left[\delta_{m, 1}+\delta_{m,-1}\right]
$$

$$
\left.\underline{N}_{m n}^{\prime \prime *} \cdot \underline{a}_{x^{\prime \prime}}\right]_{\theta^{\prime \prime}=0}=-\frac{\sqrt{n(n+1)}}{2} \frac{1}{k r^{\prime \prime}} \frac{\partial}{\partial r^{\prime \prime}}\left[r^{\prime \prime} j_{n}\left(k r^{\prime \prime}\right)\right]\left(\delta_{m, 1}-\delta_{m,-1}\right) .
$$

Consequently, eq (7.3) for the electric field vector becomes 


$$
\begin{aligned}
\underline{E}_{x}(\underline{r})=\frac{I d l \omega \mu k}{4 \pi} \sum_{n=1}^{\infty} \frac{n+1 / 2}{\sqrt{n(n+1)}}\left[\frac{\frac{\partial}{\partial r_{0}}\left[r_{0} j_{n}\left(k r_{0}\right)\right]}{k r_{0}}\left(\underline{N}_{1, n}^{(1)}-\underline{N}_{-1, n}^{(1)}\right)\right. \\
\left.\quad-i j_{n}\left(k r_{0}\right)\left(\underline{M}_{1, n}^{(1)}+\underline{M}_{-1, n}^{(1)}\right)\right], \quad r>r_{0} .
\end{aligned}
$$

From inspection of eq (7.8), we have the modal-expansion coefficients,

$$
Q_{x}^{21 n}=-Q_{x}^{2,-1, n}=\frac{I d \ell \omega \mu k}{4 \pi} \frac{n+1 / 2}{\sqrt{n(n+1)}} \frac{1}{k r_{0}} \frac{\partial}{\partial r_{0}}\left[r_{0} j_{n}\left(k r_{0}\right)\right]
$$

and

$$
Q_{x}^{11 n}=Q_{x}^{1,-1, n}=-i \frac{I d \ell \omega \mu k}{4 \pi} \frac{n+1 / 2}{\sqrt{n(n+1)}} j_{n}\left(k r_{0}\right) .
$$

The $\mathrm{x}$ subscript is used here to signify an $\mathrm{x}$-directed dipole source.

In an entirely analogous fashion, the electric field vector for a y-directed dipole source can be written

$$
\begin{aligned}
\underline{E}_{y}(\underline{r})=\frac{\omega \mu k I d l}{4 \pi} \sum_{n=1}^{\infty} \frac{n+1 / 2}{\sqrt{n(n+1)}}\left[-i \frac{\frac{\partial}{\partial r_{0}}\left[r_{0} j_{n}\left(k r_{0}\right)\right]}{k r_{0}}\left(\underline{N}_{1, n}^{(1)}+\underline{N}_{-1, n}^{(1)}\right)\right. \\
\left.\quad-j_{n}\left(k r_{0}\right)\left(\underline{M}_{1, n}^{(1)}-\underline{M}_{-1, n}^{(1)}\right)\right], \quad r>r_{0} .
\end{aligned}
$$

Consequently, we have for the modal-expansion coefficients,

$$
Q_{y}^{21 n}=Q_{y}^{2,-1, n}=-i \frac{I d l \omega \mu k}{4 \pi} \frac{n+1 / 2}{\sqrt{n(n+1)}} \frac{1}{k r_{0}} \frac{\partial}{\partial r_{0}}\left[r_{0} j_{n}\left(k r_{0}\right)\right] \text {, }
$$

and

$$
Q_{y}^{11 n}=-Q_{y}^{1,-1, n}=-\frac{I d \ell \omega \mu k}{4 \pi} \frac{n+1 / 2}{\sqrt{n(n+1)}} j_{n}\left(k r_{0}\right) .
$$

From ref. [1] we readily obtain the following limiting expressions for the radial functions

$$
\lim _{r_{0} \rightarrow 0} j_{n}\left(k r_{0}\right)=0, \quad \lim _{r_{0} \rightarrow 0} \frac{1}{k r_{0}} \frac{\partial}{\partial r_{0}}\left[r_{0} j_{n}\left(k r_{0}\right)\right]=\frac{2}{3} \delta_{l n} .
$$


Consequently, we find that all of the modal-expansion coefficients for an $\mathrm{x}$ - or $\mathrm{y}$-directed dipole, centered at the coordinate-system origin, are equal to zero except for

$$
Q_{x}^{211}=-Q_{x}^{2,-1,1}=\frac{I d \ell \omega \mu k}{8 \pi} \sqrt{2}
$$

and

$$
Q_{y}^{211}=Q_{y}^{2,-1,1}=-i \frac{I d \ell \omega \mu k}{8 \pi} \sqrt{2} .
$$

It is interesting to calculate the electric field vectors corresponding to the modalexpansion coefficients of eqs (7.13) and (7.14). Thus, at large radial distances we obtain

$$
\begin{aligned}
& \underline{E}_{x} \sim i \frac{I d l \omega \mu}{4 \pi} \frac{e^{i k r}}{r}\left(\underline{a}_{\theta} \cos \theta \cos \phi-\underline{a}_{\phi} \sin \phi\right) \\
& \underline{E}_{y} \sim i \frac{I d l \omega \mu}{4 \pi} \frac{e^{i k r}}{r}\left(\underline{a}_{\theta} \cos \theta \sin \phi+\underline{a}_{\phi} \cos \phi\right) .
\end{aligned}
$$

If $\Omega_{\mathrm{x}}$ designates the angle between the x-axis and the radial position vector $r$, and if $\Omega_{\mathrm{y}}$ designates the angle between the $y$-axis and the radial position vector $\boldsymbol{r}$, then via elementary geometry it can be shown that eqs (7.15) and (7.16) reduce to

and

$$
\underline{E}_{x} \sim-i \frac{I d l \omega \mu}{4 \pi} \frac{e^{i k r}}{r} \sin \Omega_{x} \underline{a}_{\Omega_{x}}
$$

$$
\underline{E}_{y} \sim-i \frac{I d \ell \omega \mu}{4 \pi} \frac{e^{i k r}}{r} \sin \Omega_{y} \underline{a}_{\Omega_{y}} .
$$

These final expressions for the dipole field are in complete agreement with expressions normally cited in the literature (for example, as in [42]).

\subsection{Equality between the Transverse Radiated Field and the Antenna-Dipole Coupling Equation: Receiving Electric Dipole Case}

We now establish that the radiated field of an antenna is equivalent to the signal received by an ideal dipole located at the observation point. We shall consider both $\mathrm{x}$ - and $\mathrm{y}$-directed receiving dipoles. We obtain $x$-directed dipole receiving coefficients in the doubly primed antenna's coordinate system from eqs (6.34) and (7.13) as

We introduce the notation

$$
{ }_{x} R_{211}^{\prime \prime}=-{ }_{x} R_{2,-1,1}^{\prime \prime}=-\frac{1}{3 \sqrt{2}} \frac{I d \ell}{a_{0}^{\prime \prime} \hat{\eta}_{0}^{\prime \prime}} .
$$




$$
c=\frac{I d \ell}{a_{0}^{\prime \prime} \hat{\eta}_{0}^{\prime \prime}}
$$

wherc $\mathrm{c}$ has the dimensions of unit length. Consequently, inserting eq (7.17) into cq (5.3), we obtain translated $\mathrm{x}$-dirceted dipole recciving coefficients

$$
\begin{array}{ll}
\hat{R}_{1 \mu n}^{\prime}=-i \frac{c}{4} \sqrt{n(n+1)} h_{n}^{(1)}(k d), & \mu= \pm 1 \\
\hat{R}_{2 \mu n}^{\prime}=-\mu \frac{c}{4} \sqrt{n(n+1)} \frac{1}{k d} \frac{\partial}{\partial d}\left[d h_{n}^{(1)}(k d)\right], & \mu= \pm 1
\end{array}
$$

where we have used eqs (4.31) and (4.32) in eq (5.12), and where $d$ is the separation distance.

The equivalent generator-source wave, corresponding to the signal received by an ideal $x$ directed dipole, ean be expressed according to eq (6.25) as

$$
{ }_{x} b_{G}^{\prime \prime}(\phi, \theta, \chi)=\sum_{s n m} \sum_{\mu=-1}^{1} Q^{s m n} \hat{R}_{s \mu n}^{\prime} e^{i m \phi} d_{m \mu}^{n}(\theta) e^{i \mu \chi} .
$$

We next substitute the rotation-coefficient relations (4.22), expressed here as

$$
d_{m,+1}^{n}(\theta)=\frac{-1}{\sqrt{n(n+1)}}\left[\frac{m d_{m o}^{n}(\theta)}{\sin \theta} \pm \frac{\partial d_{m o}^{n}(\theta)}{\partial \theta}\right]
$$

into cq (7.19) along with eq (7.18) to obtain, for the case $\chi=0^{\circ}$,

$$
b_{G}^{\prime \prime}(\phi, \theta, \chi=0)=\frac{c}{2} \sum_{n m}\left(Q^{1 m n} h_{n}^{(1)}(k d) \frac{i m d_{m 0}^{n}(\theta)}{\sin \theta}+Q^{2 m n} \frac{1}{k d} \frac{\partial}{\partial d}\left[d h_{n}^{(1)}(k d)\right] \frac{\partial d_{m o}^{n}(\theta)}{\partial \theta}\right) e^{i m \phi}
$$

Similarly, for the case $\chi=90^{\circ}$, we obtain

$$
b_{G}^{\prime \prime}\left(\phi, \theta, \chi=\frac{\pi}{2}\right)=-\frac{c}{2} \sum_{n m}\left(Q^{1 m n} h_{n}^{(1)}(k d) \frac{\partial d_{m 0}^{n}(\theta)}{\partial \theta}-Q^{2 m n} \frac{1}{k d} \frac{\partial}{\partial d}\left[d h_{n}^{(1)}(k d)\right] \frac{i m d_{m o}^{n}(\theta)}{\sin \theta}\right) e^{i m \phi} .
$$

Combining the two preceding equations with the defining eqs (2.31), (2.32) and (2.41) we obtain

$$
b_{x}^{\prime \prime}(\phi, \theta, \chi=0) \underline{a}_{\theta}+b_{x}^{\prime \prime}\left(\phi, \theta, \chi=\frac{\pi}{2}\right) \underline{a}_{\phi}=\frac{c}{2} \sum_{s n m} Q^{s m n} R_{s n}^{(1)}(d) \underline{G}_{n}^{s m}(\theta, \phi),
$$

where we have also made use of cq (4.3). We see that the right side of eq (7.21) corresponds to the expression for the radiated electric field in eq (2.42).

Next, we consider the case of the y-directed clementary-dipole receiving antenna. From eqs (6.34) and (7.14) we obtain the receiving coefficients of a y-directed dipole.

$$
{ }_{y} R_{211}^{\prime \prime}={ }_{y} R_{2,-1,1}^{\prime \prime}=i \frac{c}{3 \sqrt{2}} .
$$


The translated y-directed dipole receiving coefficients are then obtained by inserting eqs (4.31), (4.32), and (7.22) in eq (5.3), resulting in

$$
\begin{array}{ll}
\hat{R}_{1 \mu n}^{\prime}=-\mu \frac{c}{4} \sqrt{n(n+1)} h_{n}^{(1)}(k d), & \mu= \pm 1 \\
\hat{R}_{2 \mu n}^{\prime}=i \frac{c}{4} \sqrt{n(n+1)} \frac{1}{k d} \frac{\partial}{\partial d}\left[d h_{n}^{(1)}(k d)\right], & \mu= \pm 1
\end{array}
$$

The equivalent generator-source wave at the terminals of a y-directed dipole is given by

$$
b_{G}^{\prime \prime}(\phi, \theta, \chi)=\sum_{s n m} Q^{s i n n} \sum_{u=-1}^{1} \hat{R}_{s \mu n}^{\prime} e^{i m \phi} d_{m \mu}^{n}(\theta) e^{i \mu \chi}
$$

Consequently, upon inserting eqs (7.20) and (7.23) into eq (7.24), we obtain, for the case $\chi=0^{\circ}$,

$$
b_{G}^{\prime \prime}(\phi, \theta, \chi=0)=\frac{c}{2} \sum_{n m}\left(Q^{1 m n} h_{n}^{(1)}(k d) \frac{\partial d_{m 0}^{n}(\theta)}{\partial \theta}-Q^{2 m n} \frac{1}{k d} \frac{\partial}{\partial d}\left[d h_{n}^{(1)}(k d)\right] \frac{i m d_{m o}^{n}(\theta)}{\sin \theta}\right) e^{i m \phi},
$$

while for the case $\chi=90^{\circ}$, we have

$$
y_{G}^{\prime \prime}\left(\phi, \theta, \chi=\frac{\pi}{2}\right)=\frac{c}{2} \sum_{n m}\left(Q^{1 m n} h_{n}^{(1)}(k d) \frac{i m d_{m 0}^{n}(\theta)}{\sin \theta}+Q^{2 m n} \frac{1}{k d} \frac{\partial}{\partial d}\left[d h_{n}^{(1)}(k d)\right] \frac{\partial d_{m o}^{n}(\theta)}{\partial \theta}\right) e^{i m \phi} .
$$

Combining the two preceding equations and again using eqs (2.31), (2.32) and (2.41) with eq (4.3) results in

$$
{ }_{y} b_{G}^{\prime \prime}\left(\phi, \theta, \chi=\frac{\pi}{2}\right) \underline{a}_{\theta}-{ }_{y} b_{G}^{\prime \prime}(\phi, \theta, \chi=0) \underline{a}_{\phi}=\frac{c}{2} \sum_{s n m} Q^{s m n} R_{s n}^{(1)}(d) \underline{G}_{n}^{s m}(\theta, \phi) .
$$

As before, the right side of eq (7.25) corresponds to the radiated electric field in eq (2.42).

An alternate way to express the results (7.21) and (7.25) is obtained by using a dotproduct vector formulation. Thus, the vector dipole moment for an $\mathrm{x}$-directed dipole is

$$
\vec{p}_{x}=\operatorname{Idl}\left(\underline{a}_{\theta} \cos \chi+\underline{a}_{\phi} \sin \chi\right)
$$

while for the $y$-directed dipole wc have

$$
\vec{p}_{y}=\operatorname{Id} \ell\left(\underline{a}_{\theta} \sin \chi-\underline{a}_{\phi} \cos \chi\right)
$$

Using these definitions, the expressions (7.21) and (7.25) can be combined to obtain

$$
b_{G}^{\prime \prime}=\frac{1}{2 a_{0}^{\prime \prime} \hat{\eta}_{0}^{\prime \prime}} \sum_{s n m} Q^{s m n} R_{s n}^{(1)}(d) \underline{G}_{n}^{s i n}(\theta, \phi) \cdot \vec{p},
$$

wherc the dipole moment $\vec{p}$ is given either by eq (7.26a) or (7.26b) for the respective cases $b_{G}^{\prime \prime} \equiv{ }_{x} b_{G}^{\prime \prime}$ or $b_{G}^{\prime \prime} \equiv_{y} b_{G}^{\prime \prime}$. Note that the angle $\chi$ rcpresents a rotation of the doubly primed coordinate system about the $\mathrm{z}^{\prime \prime}$ axis, while changing from an $\mathrm{x}$ - to a $\mathrm{y}$-directed dipole corresponds to an inverse rotation by $90^{\circ}$ about the $z^{\prime \prime}$ axis. Therefore, we have the rclation $\left.\vec{p}_{\chi}\right]_{\chi \rightarrow \chi-\frac{\pi}{2}}=\vec{p}_{y}$. 
In the next section, we will comparc eq (7.27) to the corresponding result obtained for a transmitting ideal dipole and a reciprocal receiving antenna.

\subsection{Transmitting Electric Dipole Case}

Next, we consider the case of a transmitting elementary dipole in place of the doubly primed antenna, and we show that the two-antenna coupling for this case is compatible with the received signal due to a dipole source. That is, we compare the result of assuming a dipole source in eq (5.22) with the generator-source wave that would be produced by the incident ficld of eq (2.27).

We require expressions for $\alpha_{s \mu n}^{\sigma v}$ similar to the particular case expressions that were obtaincd for $C_{\sigma \mu \nu}^{s n}$ from (4.31) and (4.32). Combining eqs (4.30) and (6.9), we obtain

$$
a(1,1,-1, n, n+1)=-a(1,1,-1, n, n-1)=\frac{1}{2 n+1}
$$

Conscquently, for the particular case $v=1$ the general formulas (6.4) reduce to

$$
a_{l n}^{11}=a_{-l n}^{-11}=\frac{2 n+1}{\sqrt{2 n(n+1)}} \frac{1}{k d} \frac{\partial}{\partial d}\left[d h_{n}^{(1)}(k d)\right]
$$

and

$$
b_{l n}^{11}=-b_{-l n}^{-11}=-i \frac{2 n+1}{\sqrt{2 n(n+1)}} h_{n}^{(1)}(k d) .
$$

Substituting eq (7.13) into (6.33) gives us the mode coefficients for an $x$-directed dipole in the doubly primed coordinate system

$$
\hat{Q}_{x}^{/ / 211}=-\hat{Q}_{x}^{/ / 2,-1,1}=-\frac{I d \ell \omega \mu k}{8 \pi} \sqrt{2} .
$$

Conscquently, combining eqs (7.28) and (7.29) into eq (5.16) results in the translated mode coefficicnts

$$
{ }_{x} \hat{P}_{1,1, n}^{\prime}={ }_{x} \hat{P}_{1,-1, n}^{\prime}=i \frac{\operatorname{Id} l \omega \mu k}{4 \pi} \frac{n+1 / 2}{\sqrt{n(n+1)}} h_{n}^{(1)}(k d),
$$

and

$$
{ }_{x}^{\prime} \hat{P}_{2,1, n}^{\prime}=-\hat{P}_{2,-1, n}^{\prime}=-\frac{I d \ell \omega \mu k}{4 \pi} \frac{n+1 / 2}{\sqrt{n(n+1)}} \frac{1}{k d} \frac{\partial}{\partial d}\left[d h_{n}^{(1)}(k d)\right] .
$$

Let us introduce the simplifying notation

$$
b=\frac{I d \ell \omega \mu k}{4 \pi} .
$$


Then, using eqs (7.20) and (7.30) in (5.20), we obtain the modal expansion coefficients for an $\mathrm{x}$-directed dipole source referred to the unprimed or receiving antenna's coordinate system

$$
\begin{aligned}
{ }_{x} \hat{P}_{1 m n} & =b \frac{2 n+1}{n(n+1)} h_{n}^{(1)}(k d) e^{-i m \phi}\left(\frac{-i m d_{m 0}^{n}(\theta)}{\sin \theta} \cos \chi-\frac{\partial d_{m 0}^{n}(\theta)}{\partial \theta} \sin \chi\right) \\
& =b \frac{2 n+1}{n(n+1)} h_{n}^{(1)}(k d) \underline{G}_{n}^{1 m *}(\theta, \phi) \cdot\left(\underline{a}_{\theta} \cos \chi+\underline{a}_{\phi} \sin \chi\right) .
\end{aligned}
$$

and

$$
\begin{aligned}
{ }_{x} \hat{P}_{2 m n} & =b \frac{2 n+1}{n(n+1)} \frac{1}{k d} \frac{\partial}{\partial d}\left[d h_{n}^{(1)}(k d)\right] e^{-i m \phi}\left(\frac{-i m d_{m 0}^{n}(\theta)}{\sin \theta} \sin \chi+\frac{\partial d_{m 0}^{n}(\theta)}{\partial \theta} \cos \chi\right) \\
& =b \frac{2 n+1}{n(n+1)} \frac{1}{k d} \frac{\partial}{\partial d}\left[d h_{n}^{(1)}(k d)\right] \underline{G}_{n}^{2 m *}(\theta, \phi) \cdot\left(\underline{a}_{\theta} \cos \chi+\underline{a}_{\phi} \sin \chi\right) .
\end{aligned}
$$

As expected, eqs (7.31) are identical to the coefficients of the vector spherical-wave functions in eq (2.27).

Repeating the above analysis for a y-directed dipole, we obtain

$$
\begin{gathered}
{ }_{y} \hat{P}_{1 m n}=b \frac{2 n+1}{n(n+1)} h_{n}^{(1)}(k d) \underline{G}_{n}^{1 m *}(\theta, \phi) \cdot\left(\underline{a}_{\theta} \sin \chi-\underline{a}_{\phi} \cos \chi\right) \\
\hat{P}_{2 m n}=b \frac{2 n+1}{n(n+1)} \frac{1}{k d} \frac{\partial}{\partial d}\left[d h_{n}^{(1)}(k d)\right] \underline{G}_{n}^{2 m *}(\theta, \phi) \cdot\left(a_{\theta} \sin \chi-\underline{a}_{\phi} \cos \chi\right) .
\end{gathered}
$$

We see that eqs (7.32) corresponds to eqs (7.31) with $\chi$ replaced by $\chi-(\pi / 2)$.

The generator-source wave at the receiving antenna, due to the dipolc source, is obtained using either eqs (7.31) or (7.32) in (5.21). Introducing a dot-product vector formulation, we obtain a combincd cxpression applicable to cither an $\mathrm{x}$ - or $\mathrm{y}$-directed transmitting dipole source,

$$
b_{G}=\frac{\omega \mu k}{4 \pi} \sum_{s n m} \frac{2 n+1}{n(n+1)} R_{s n}^{(1)}(d) \underline{G}_{n}^{s m *}(\theta, \phi) \cdot \vec{p} R_{s m n},
$$

where the dipole moment $\vec{p}$ is given by either eq (7.26a) or (7.26b).

It is intercsting to consider the effect of assuming a reciprocal receiving antenna.

Substituting the reciprocity relation (3.8) into (7.33), reversing the order of the $m$ summation, and using eq (2.30), we obtain

$$
b_{G}=\frac{1}{2 \hat{\eta}_{0}} \sum_{s n m} T^{s m n} R_{s n}^{(1)}(d) \underline{G}_{n}^{s m}(\theta, \phi) \cdot \vec{p},
$$

a result equivalent to eq (7.27). 


\subsection{Conclusions}

This chapter starts by developing modal-expansion coefficients for an ideal-dipole source located along the z-axis (eqs (7.9) and (7.11)). Specializing to a receiving dipole at the doubly primed coordinate system's origin and translating coordinates, we obtain translated-dipole receiving coefficients in the test-antenna coordinate system (eqs (7.18) and (7.23)). Inserting these results into our two-antenna coupling equations, we obtain for $\chi=0$ and $\chi=(\pi / 2)$ the transverse components of the radiated electric field (eqs (7.21) and (7.25)), as would be expected. From these results, we go on to express the signal received by an ideal dipole as a dot product between the vector dipole moment and the radiated electric field (eq (7.27)). This result is subsequently used as a reciprocal case comparison when the direction of propagation is reversed.

Reversing the propagation direction and using our previous expression for an ideal-dipole source, we obtain exciting modal-expansion coefficients for the field about the test antenna (eqs (7.31) and (7.32)) that are identical to similar results obtained in Chapter II (eq (2.27)), where a dyadic Green's function representation of an external dipole source was given. We use these results to express the received signal at the test antenna as a dot product between the dipole moment and the modal excitation vector (eq (7.33)). Applying the reciprocity relation to eq (7.33) we obtain eq (7.34), which is seen to be equivalent to the receiving dipole expression obtained earlier (eq (7.27)). Thus, in this chapter we have demonstrated that the expressions introduced in Chapter II to describe antenna fields are compatible with the antenna-coupling equations developed in Chapter $\mathrm{V}$.

In Chapter IX, we shall make use of some of the expressions developed here in devising numerical comparison tests to check the validity of the procedure implementing the sphericalscanning algorithm that will be developed in Chapter VIII. Also in Chapter IX we shall show that the integral expressions to be given in Chapter VIII for the modal-expansion coefficients $\mathrm{Q}^{\mathrm{smn}}$, in the case where the probe is an ideal dipole, are equivalent to applying the orthogonality relations (2.39) between vector spherical-wave functions to the expressions given in Chapter II for the radiated electric field. In the meantime, we turn our attention in the next chapter to dcveloping an efficient algorithm for near-field spherical scanning. 


\section{CHAPTER VIII}

\section{SPHERICAL SCANNING}

\subsection{Introduction}

Spherical scanning consists of measuring either the transmitted or received signal at discrete lattice points on the surface of a sphere enclosing the test antenna, using an electrically small probe antenna that travels over the surface of the sphere to each lattice point. Generally, the probe antenna is in the near field of the test antenna. The goal of the data processing problem is to determine the modal-expansion coefficients of the field from the measured data and to subsequently determine the far-field pattern and gain of the test antenna from these expansion coefficients. Usually, both data processing and actual data measurement are linked together in the terminology.

As indicated in Chapter I, this work defers to the excellent survey of the previous work on probe-corrected near-field analysis given by Kerns $[25,26]$. However, it is worth emphasizing that the first nonplanar near-field scanning analysis was carried out by Brown and Jull [8], who limited their scanning analysis to two-dimensional fields. The background for plane-wave angular-spectrum analysis was set by Booker and Clemmow [5]. Their formulation was applied by Brown [7] in connection with estimating antenna measurement errors, and plane-wave spectrum analysis forms the basis for the treatment by Kerns and Dayhoff [28] upon which Kerns' planar scanning analysis [26] is based.

A major reason for the interest in near-field scanning is the high measurement accuracy that is possible with these techniques [22]. Indeed, Kerns [26] notes that "the accuracy of the near-field measurement techniques equals, or in some cases exceeds, that achieved with the best far-field ranges, and more complete data are generally obtained." The high accuracy that is theoretically obtainable with near-field techniques could eventually lead to these methods forming the standard against which other antenna measurement techniques are compared. As Kummer and Gillespie [30] note, "the near-field techniques may well become accepted as the most accurate technique for the measurement of power gain and of patterns for antennas that can be accommodated by the measuring apparatus." An assessment of near-field measurement work that has been carried out at various research organizations is given in a report by Ashton, et al. [2]. 
The basic equation for carrying out spherical near-field scanning is the antenna-coupling equation (5.6), which was derived in Chapter V. A slightly modified version of this equation was originally formulated by Jensen [21]. The orthogonality of the spherical-point-group representation coefficients, upon integration ovcr the surface of the measurcment sphere, is the next most important factor in the analysis. This orthogonality was specifically noted by Jensen, but was rejected in favor of a matrix inversion technique. The use of modal coefficient decoupling, using the orthogonality relationship in order to achieve efficient data processing, forms the basis for the technique proposed by Wacker [54]. At the same time, Wacker proposed using a circularly symmetric probe, which would reduce one of the required integrations to a two-term sum. The remaining numerical integrations would be carried out using fast Fourier transform (FFT) processing, according to Wacker's formulation [55]. The translation of the probe-antenna coefficients to the test antenna's coordinate system (see eq (5.3)) was proposed [55] to be carried out prior to decoupling of the modal-expansion coefficients.

In his report on data processing techniques in connection with spherical scanning, Wacker [56] proposed both "no probe correction" and full probe-compensated data processing. However, Chapter VII showed that "no probe correction" was just a special case of probe correction analysis. As indicated in Chapter I, Wacker's predominantly matrix cquation analysis [56] will be recast in scalar equation form. Then, new developments to make the data processing more efficient will be introduced. In Section 8.2.1 we present an introductory basic development of spherical-scanning modal coefficient decoupling. In Section 8.2.2 we specialize to the case of discrete data sampling points and carry out the modal coefficient decoupling as far as Wacker's development carried it. The development of equations for an improved computational algorithm is presented in Section 8.2.3. Then, in Section 8.3, we present a synopsis of the relevant computational steps comprising our spherical near-field scanning algorithm. Finally, in Section 8.4 we present an additional simplification of the data-processing effort when the test-antenna's back-hemisphere radiation is negligible.

\subsection{Probe-Correction Case Formulation for Spherical Scanning Assuming a Transmitting Test Antenna and Receiving Probe}

\subsubsection{Basic Theoretical Development}

For this initial theoretical development, the signal received by a known probc antenna is assumed to be given everywhere on the surface of a hypothetical measurement spherc. The problem geometry is depicted in figure 5.1 for a particular orientation $\phi_{0}, \theta_{0}, \chi$ of the probe 
relative to the test-antenna coordinate system. In what follows, we shall drop the zero subscript on these Euler angles describing the probe-antenna's position. The antenna on the right in figure 5.1 is the probe, while the antenna on the left is the antenna under test. The separation distance $d$ corresponds to the fixed radius of the measurement sphere. The starting equation for probe-compensated spherical-scanning in the case of a transmitting test antenna is given by eq (5.6) as

$$
b_{G}^{\prime \prime}(\phi, \theta, \chi)=\sum_{s=1}^{2} \sum_{n=1}^{\infty} \sum_{m=-n}^{n} \sum_{\mu=-n}^{n} Q^{s m n} \hat{R}_{s \mu n}^{\prime} D_{m \mu}^{n *}(\phi, \theta, \chi) .
$$

Here, it is implicitly assumed that the modal-expansion coefficients $Q^{\mathrm{smn}}$ are invariant with respect to the probe position angles $\phi, \theta, \chi$; this will be true, for instance, if multiple reflections between the probe and test antenna are negligible. The preceding equation expresses the equivalent generator-source wave at the receiving probe antenna for any position $\theta, \phi$ on the surface of the measurement sphere for an arbitrary probe-antenna rotation angle $\chi$. In eq (8.1), it is assumed that $b_{G}^{\prime \prime}$ is a known function of the position coordinates and that the translated probe coefficients $\hat{R}_{s \mu n}^{\prime}$ are also known. We want to evaluate the unknown modal-expansion coefficients $\mathrm{Q}^{\mathrm{smn}}$. Then, using eq (3.3) to relate $\mathrm{T}^{\mathrm{smn}}$ to $\mathrm{Q}^{\mathrm{smn}}$, the gain of the test antenna can be calculated using eq (3.15).

The relation (see eq (4.2))

$$
D_{m \mu}^{n *}(\phi, \theta, \chi)=e^{i m \phi} d_{m \mu}^{n}(\theta) e^{i \mu \chi}
$$

shows that the left side of eq (8.1) can be considered as a two-dimensional Fourier series in $\phi$ and $\chi$. A solution for $\mathrm{Q}^{\mathrm{smn}}$ can be found by expanding eq (8.1) into a Fourier series, thus permitting $\mathrm{Q}^{\mathrm{smn}}$ to be resolved in terms of Fourier-series expansion coefficients. We have

$$
b_{G}^{\prime \prime}(\phi, \theta, \chi)=\sum_{m=-\infty}^{\infty} \sum_{\mu=-\infty}^{\infty} e^{i m \phi} b_{m}^{\mu}(\theta) e^{i \mu \chi}
$$

where

$$
b_{m}^{\mu}(\theta)=\frac{1}{4 \pi^{2}} \int_{0}^{2 \pi} d \phi \int_{0}^{2 \pi} d \chi b_{G}^{\prime \prime}(\phi, \theta, \chi) e^{-i(m \phi+\mu \chi)} .
$$

Equation (8.1) may be written 


$$
\sum_{m \mu} e^{i m \phi} b_{m}^{\mu}(\theta) e^{i \mu \chi}=\sum_{s n m} \sum_{\mu} Q^{s m n} \hat{R}_{s \mu n}^{\prime} e^{i m \phi} d_{m \mu}^{n}(\theta) e^{i \mu \chi} .
$$

As can be shown by the orthogonality relations for Fourier series, the coefficients of $\mathrm{e}^{\mathrm{i}(\mathrm{m} \phi+\mu \chi)}$ on the two sides of this equation must be equal. Therefore,

$$
b_{m}^{\mu}(\theta)=\sum_{s=1}^{2} \sum_{n=(1,|m|,|\mu|)}^{\infty} Q^{s m n} \hat{R}_{s \mu n}^{\prime} d_{m \mu}^{n}(\theta) .
$$

Here, similar to eq (4.23), the notation $(1,|\mathrm{~m}|,|\mu|)$ denotes the larger of $1,|\mu|$, or $|\mathrm{m}|$. Our result (8.5) was arrived at by a Fourier series expansion in $\phi$ and $\chi$. A further step toward solution can be achieved by expansion in terms of the orthogonal set of functions $d_{m \mu}^{n}(\theta)$ and use of the orthogonality relation ([44], eq (4.60), or [17], eq (4.6.1))

$$
\int_{0}^{\pi} d_{m \mu}^{\ell}(\theta) d_{m \mu}^{n}(\theta) \sin \theta d \theta=\frac{2}{2 n+1} \delta_{n \ell} .
$$

Thus, the expansion of eq (8.1) is

$$
\sum_{m \mu n} b_{m n}^{\mu} D_{m \mu}^{n *}(\phi, \theta, \chi)=\sum_{s n m} \sum_{\mu} Q^{s m n} \hat{R}_{s \mu n}^{\prime} D_{m \mu}^{n *}(\phi, \theta, \chi),
$$

where

$$
b_{m n}^{\mu}=\frac{2 n+1}{2} \int_{0}^{\pi} b_{m}^{\mu}(\theta) d_{m \mu}^{n}(\theta) \sin \theta d \theta \text {. }
$$

Equation (8.6) is obtained from the more general orthogonality relation [17]

$$
\begin{gathered}
\frac{1}{8 \pi^{2}} \int_{0}^{2 \pi} d \phi \int_{0}^{\pi} d \theta \int_{0}^{2 \pi} d \chi D_{m^{\prime} m}^{\ell *}(\phi, \theta, \chi) D_{\mu^{\prime} \mu}^{n}(\phi, \theta, \chi) \sin \theta \\
=\frac{1}{2 n+1} \delta_{m^{\prime} \mu^{\prime}} \delta_{m \mu} \delta_{\ell n} .
\end{gathered}
$$

This orthogonality relation imposes the requirement that the coefficients on the two sides of the equality (8.7) must be equal, so that

$$
b_{m n}^{\mu}=\sum_{s=1}^{2} Q^{s m n} \hat{R}_{s \mu n}^{\prime} .
$$

Now, from eqs (8.4) and (8.8), we can calculate the series expansion coefficients $b_{m n}^{\mu}$ on the left side of eq (8.9) using a particular probe. A least-squares solution of the system of eqs (8.9) that 
involve just the two modal-expansion coefficients $\mathrm{Q}^{\mathrm{mmn}}$ and $\mathrm{Q}^{2 \mathrm{mn}}$ would enable us to solve for these quantities ([24], p. 92).

Proceeding in this fashion for each applicable value of the $\mathrm{m}$ and $\mathrm{n}$ indices, we could obtain the entire set of modal-expansion coefficients $Q^{\mathrm{smn}}$ characterizing the radiated field of the test antenna. Then eqs (2.15) or (2.42) could be used to obtain the radiated electric field at any distance $r$ beyond the measurement sphere. An alternative procedure is to substitute the set of modal-expansion coefficients that have been obtained into eqs (5.6) or (8.1) in order to obtain the signal received by some known probe antenna located at an arbitrary distance $r$ beyond the measurement sphere. In particular, we could evaluate eq (5.6) for an ideal-dipole probe located in the far field of the test antenna. The resulting evaluation for the received signal would be proportional to the far-zone electric field radiated by the test antenna in that direction. It should be noted that the probe used to collect the data over the surface of the measurement sphere would most likely differ greatly from the hypothetical probe used to calculate the far-field signal.

Equations (8.4) and (8.8) show that our formal solution requires a knowledge of the received signal at each point $\theta, \phi$ on the measurement sphere as a function of the probe rotation angle $\chi$. The approach proposed by Wacker [55] to eliminate one of these three required integrations was to use a circularly symmetric probe, so that the only nontrivial probe coefficients would be those corresponding to $\mu= \pm 1$. Then eq (8.1) would become

$$
b_{G}^{\prime \prime}(\phi, \theta, \chi)=\sum_{s n m} Q^{s m n} \sum_{\mu=-1}^{1} \hat{R}_{s \mu n}^{\prime} e^{i m \phi} d_{m \mu}^{n}(\theta) e^{i \mu \chi},
$$

where the prime on the summation sign over $\mu$ is used to indicate that the $\mu=0$ term is excluded.

Now, from eq (8.10) we can write the two-term Fourier series expansion

$$
b_{G}^{\prime \prime}(\phi, \theta, \chi)=b^{-1}(\phi, \theta) e^{-i \chi}+b^{+1}(\phi, \theta) e^{i \chi},
$$

where

$$
b^{\mu}(\phi, \theta)=\frac{1}{2 \pi} \int_{0}^{2 \pi} b_{G}^{\prime \prime}(\phi, \theta, \chi) e^{-i \mu \chi} d \chi ; \quad \mu= \pm 1 .
$$

In particular, we can evaluate eq (8.11) at the specific angles $\chi=0$ and $\chi=\pi / 2$ to obtain

$$
b_{G}^{\prime \prime}(\phi, \theta, \chi=0)=b^{-1}(\phi, \theta)+b^{+1}(\phi, \theta)
$$

and

$$
b_{G}^{\prime \prime}\left(\phi, \theta, \chi=\frac{\pi}{2}\right)=-i b^{-1}(\phi, \theta)+i b^{+1}(\phi, \theta) .
$$


The preceding pair of equations can be solved for the Fourier-series coefficients $b^{\mu}(\phi, \theta)$, where $\mu= \pm 1$. Then, from eq (8.12) we obtain

$$
\frac{1}{2 \pi} \int_{0}^{2 \pi} b_{G}^{\prime \prime}(\phi, \theta, \chi) e^{-i \mu \chi} d \chi=\frac{1}{2}\left[b_{G}^{\prime \prime}(\phi, \theta, \chi=0)-i \mu b_{G}^{\prime \prime}\left(\phi, \theta, \chi=\frac{\pi}{2}\right)\right], \quad \mu= \pm 1 .
$$

We can now substitute eq (8.13) into eq (8.4), resulting in

$$
b_{m}^{\mu}(\theta)=\frac{1}{4 \pi} \int_{0}^{2 \pi}\left[b_{G}^{\prime \prime}(\phi, \theta, \chi=0)-i \mu b_{G}^{\prime \prime}\left(\phi, \theta, \chi=\frac{\pi}{2}\right)\right] e^{-i m \phi} d \phi, \quad \mu= \pm 1 .
$$

Using eq (8.14) in place of eq (8.4) greatly simplifies the problem of determining the modalexpansion coefficients $Q^{\mathrm{smn}}$. Specifically, eq (8.9) with $\mu=+1$ and $\mu=-1$ produces just two equations to be solved for the unknown coefficients $Q^{1 m n}$ and $Q^{2 m n}$.

\subsubsection{Computation Algorithms for Discrete Data-Sampling Points}

In the preceding section we presented a theoretical development for obtaining the modalexpansion coefficients of the transmitting-test-antenna's field, assuming a known probe antenna and assuming that the received signal is known at every point on the surface of a sphere about the test antenna. Multiple reflections between the two antennas were assumed to be negligible in order to insure that the modal-expansion coefficients would be invariant with respect to probe position on the spherical surface. A considerable simplification resulted upon assuming that all probe receiving coefficients $\hat{R}_{s \mu n}^{\prime}$ are negligible except those for $\mu= \pm 1$. We now consider the problem of efficient data processing when the received signal is just known at a finite number of equally spaced discrete lattice points on the measurement sphere's surface.

With a finite amount of data only a finite number of modal-expansion coefficients can be resolved. Consequently, we assume that all modal-expansion coefficients $\mathrm{Q}^{\mathrm{smn}}$ of order $\mathrm{n}$ greater than some fixed value $\mathrm{N}$ are negligible. We formalize this assumption by introducing a new symbol, W, for the equivalent generator source wave that is generated by just a finite number of received modes. Thus,

$$
W(\phi, \theta, \chi)=\sum_{s=1}^{2} \sum_{n=1}^{N} \sum_{m=-n}^{n} Q^{s m n} \sum_{\mu=-1}^{1} \hat{R}_{s \mu n}^{\prime} e^{i m \phi} d_{m \mu}^{n}(\theta) e^{i \mu \chi} .
$$

Equation (8.15) also assumes negligible probe receiving coefficients for $\mu \neq \pm 1$, and the prime on the $\boldsymbol{\mu}$-summation sign indicates the $\boldsymbol{\mu}=0$ term is absent. Comparing eq (8.15) with (8.10) or (8.1), we see that we have made the approximation,

$$
W(\phi, \theta, \chi) \approx b_{G}^{\prime \prime}(\phi, \theta, \chi) \text {. }
$$


We now introduce the finite Fourier series expansion (4.11) for the spherical representation coefficients, which we reexpress here as

$$
d_{m \mu}^{n}(\theta)=i^{\mu-m} \sum_{m^{\prime}=-n}^{n} \Delta_{m^{\prime} m}^{n} \Delta_{m^{\prime} \mu}^{n} e^{i m^{\prime} \theta} .
$$

Substituting eq (8.16) into (8.15) and interchanging the orders of summation results in

$$
\begin{aligned}
W(\phi, \theta, \chi) & =\sum_{m=-N}^{N} \sum_{m^{\prime \prime}=-N}^{N} e^{i\left(m \phi+m^{\prime \prime} \theta\right)} \sum_{\mu=-1}^{1} e^{i \mu \chi+i(\mu-m) \frac{\pi}{2}} \\
& \times \sum_{n=\left(1,|m|,\left|m^{\prime \prime}\right|\right)}^{N} \Delta_{m^{\prime \prime} m}^{n} \Delta_{m^{\prime \prime} \mu}^{n} \sum_{s=1}^{2} Q^{s m n} \hat{R}_{s \mu n}^{\prime}
\end{aligned}
$$

Here, as in eq (8.5), the notation $\left(1,|\mathrm{~m}|,\left|\mathrm{m}^{\prime \prime}\right|\right)$ designates the larger of $1,|\mathrm{~m}|$, or $\left|\mathrm{m}^{\prime \prime}\right|$. The notation change $\mathrm{m}^{\prime \prime}$ instead of $\mathrm{m}^{\prime}$ is introduced for uniformity with subsequent expressions.

Equation (8.17) expresses the received signal $\mathrm{W}(\phi, \theta, \chi)$ as a finite Fourier series at discrete lattice points in both $\theta$ and $\phi$. Treating the finite Fourier series expansion in $\phi$ first, we have

$$
W(\phi, \theta, \chi)=\sum_{m=-N}^{N} W_{m}(\theta, \chi) e^{i m \phi}
$$

where

$$
W_{m}(\theta, \chi)=\frac{1}{2 \pi} \int_{0}^{2 \pi} W(\phi, \theta, \chi) e^{-i m \phi} d \phi
$$

Note that in many instances azimuthal symmetry exists, in which case not all of the terms in the sum (8.18) are significant. This possibility is recognized by stating that just $\mathrm{N}_{\phi}$ terms in the summation over the $m$ index in eq (8.18) are retained, where $N_{\phi} \leq 2 N$. Evaluating eq (8.18) for $\mathrm{W}(\phi, \theta, \chi)$ just at the discrete lattice points $\phi=\mathrm{k} \Delta \phi$, where $\Delta \phi=\frac{2 \pi}{N_{\phi}}, \mathrm{k}=0,1,2, \ldots, \mathrm{N}_{\phi}-1$, we obtain

$$
W(k \Delta \phi, \theta, \chi)=\sum_{m=1-\frac{N_{\phi}}{2}}^{\frac{N_{\phi}}{2}} W_{m}(\theta, \chi) e^{i m k \Delta \phi} .
$$


Equation (8.20) implicitly assumes that $N_{\phi}$ is an even integer. ${ }^{1}$ In the event that there is no azimuthal symmetry, then we have dropped at most one term in going from eq (8.18) to (8.20).

We solve the $\mathrm{N}_{\phi}$ simultaneous equations (8.20) by making use of the orthogonality relation,

$$
\sum_{k=0}^{N_{\phi}-1} e^{i q k \Delta \phi} \times e^{-i m k \Delta \phi}=N_{\phi} \delta_{q m} .
$$

We obtain

$$
W_{m}(\theta, \chi)=\frac{1}{N_{\phi}} \sum_{k=0}^{N_{\phi}-1} W(k \Delta \phi, \theta, \chi) e^{-i m k \Delta \phi},
$$

where $m=1-\frac{N_{\phi}}{2}, 2-\frac{N_{\phi}}{2}, \cdots, \frac{N_{\phi}}{2}-1, \frac{N_{\phi}}{2}$. Comparing the above discrete series expression to eq (8.19) results in

$$
\frac{1}{2 \pi} \int_{0}^{2 \pi} W(\phi, \theta, \chi) e^{-i m \phi} d \phi=\frac{1}{N_{\phi}} \sum_{k=0}^{N_{\phi}-1} W(k \Delta \phi, \theta, \chi) e^{-i m k \Delta \phi} .
$$

Equation (8.15) constitutes a finite but continuous Fourier-series expansion in the variables $\phi$ and $\chi$. Accordingly, in eqs (8.3) and (8.4) we can replace $b_{G}^{\prime \prime}(\phi, \theta, \chi)$ by $W(\phi, \theta, \chi)$ and $b_{m}^{\mu}(\theta)$ by $W_{m}^{\mu}(\theta)$ to obtain equivalent expressions which correspond to restricting $W_{m}^{\mu}(\theta)$ to a finite range on the $\mathrm{m}$ and $\mu$ indexes. Thus, we have

where

$$
W(\phi, \theta, \chi)=\sum_{m=1-\frac{N_{\phi}}{2}}^{\frac{N_{\phi}}{2}} \sum_{\mu=-1}^{1} e^{i m \phi} W_{m}^{\mu}(\theta) e^{i \mu \chi},
$$

$$
W_{m}^{\mu}(\theta)=\frac{1}{4 \pi^{2}} \int_{0}^{2 \pi} d \phi \int_{0}^{2 \pi} d \chi W(\phi, \theta, \chi) e^{-i(m \phi+\mu \chi)} .
$$

Moreover, in eq (8.14) we can also replace b's with W's to obtain an equivalent expression.

Then, upon combining that result with eq (8.21), we obtain

$$
W_{m}^{\mu}(\theta)=\frac{1}{2}\left[W_{m}^{0}(\theta)-i \mu W_{m}^{\frac{\pi}{2}}(\theta)\right], \quad \mu= \pm 1 .
$$

Here, we have introduced a special notation for the discrete Fourier transforms

${ }^{1}$ This restriction is predominantly due to a requirement that the angular encoder for data measurement be calibrated in degrees, which implies that $\mathrm{N}_{\phi}$ be equal to 360 divided by an integer. The restriction is not due solely to the FFT, inasmuch as the FFT algorithm is inherently most efficient when the number of terms summed equals 3 raised to a power [14]. 


$$
\begin{aligned}
W_{m}^{0}(\theta) & =\frac{1}{N_{\phi}} \sum_{k=0}^{N_{\phi}-1} W(k \Delta \phi, \theta, \chi=0) e^{-i m k \Delta \phi}, \\
W_{m}^{\frac{\pi}{2}}(\theta) & =\frac{1}{N_{\phi}} \sum_{k=0}^{N_{\phi}-1} W\left(k \Delta \phi, \theta, \chi=\frac{\pi}{2}\right) e^{-i m k \Delta \phi} .
\end{aligned}
$$

In eqs (8.23) and (8.24) the index $m$ takes on the values $1-\frac{N_{\phi}}{2}, 2-\frac{N_{\phi}}{2}, \cdots, \frac{N_{\phi}}{2}-1, \frac{N_{\phi}}{2}$. Now, setting eq (8.15) equal to (8.22a) and using the orthogonality relations for Fourier series gives us the equivalent of eq (8.5),

$$
W_{m}^{\mu}(\theta)=\sum_{s=1}^{2} \sum_{n=(1,|m|)}^{N} Q^{s m n} \hat{R}_{s \mu m}^{\prime} d_{m \mu}^{n}(\theta), \quad \mu= \pm 1 .
$$

Let us introduce the notation

$$
W_{m n}^{\mu}=\sum_{s=1}^{2} \hat{R}_{s \mu n}^{\prime} Q^{s m n}
$$

Using eq (8.26), eq (8.25) can be rewritten as

$$
W_{m}^{\mu}(\theta)=\sum_{n=(1,|m|)}^{N} W_{m n}^{\mu} d_{m \mu}^{n}(\theta),
$$

whence the orthogonality relation (8.6) gives us

$$
W_{m n}^{\mu}=\frac{2 n+1}{2} \int_{0}^{\pi} W_{m}^{\mu}(\theta) d_{m \mu}^{n}(\theta) \sin \theta d \theta
$$

For computational purposes, it is desirable to define $\stackrel{\circ}{n}_{n}^{m \mu}$ by the relation

$$
W_{m n}^{\mu}=\left(n+\frac{1}{2}\right) i^{m-\mu} \stackrel{\circ}{W}_{n}^{m \mu} .
$$

Then eq (8.26) can be rewritten as

$$
\frac{i^{\mu-m}}{n+\frac{1}{2}} \sum_{s=1}^{2} Q^{s m n} \hat{R}_{s \mu n}^{\prime}=\stackrel{\circ}{W}_{n}^{m \mu}
$$

Now we would like to substitute the exact finite Fourier series expansion (8.16) into (8.27). However, if we look ahead toward the computation of the far-field pattern we find that we can employ a novel method to make some exponential phase factors cancel out of the computations. Specifically, once we obtain the modal-expansion coefficients $Q^{\mathrm{smn}}$ we would like to substitute 
them back into eq (8.17), using in place of the near-field probe an ideal dipole located in the farfield of the test antenna. The method we propose will enable us to cancel out the phase factor $\mathrm{i}^{\mu-\mathrm{m}}$ that is contained in eq (8.17). All we need to do is recognize that $d_{m \mu}^{n}(\theta)$ is a real function, and so must equal its own complex conjugate. Substituting the complex conjugate of eq (8.16) into (8.27) will enable us to ultimately obtain the product term $\mathrm{i}^{-\mathrm{m}} \mathrm{i}^{\mathrm{m}}=1$ in our final expression for the far field. Carrying out the substitution while simultaneously invoking the definition (8.28) results in

$$
\stackrel{\circ}{W}_{n}^{m \mu}=\sum_{m^{\prime}=-n}^{n} \Delta_{m^{\prime} m}^{n} \Delta_{m^{\prime} \mu}^{n} \int_{0}^{\pi} W_{m}^{\mu}(\theta) e^{-i m^{\prime} \theta} \sin \theta d \theta .
$$

We now recall from eq (8.17) that the assumed received signal $\mathrm{W}(\phi, \theta, \chi)$ has a finite Fourier series expansion in $\theta$. Therefore, as a consequence of eq (8.22a) we must have

where

$$
W_{m}^{\mu}(\theta)=\sum_{m^{\prime \prime}=-N}^{N} W_{m}^{m \mu} e^{i m^{\prime \prime} \theta}
$$

$$
W_{m}^{m \mu}=\frac{1}{2 \pi} \int_{0}^{2 \pi} W_{m}^{\mu}(\theta) e^{-i m^{\prime \prime} \theta} .
$$

Let us now rewrite eq (8.31) in the form

$$
W_{m}^{\mu}(\theta)=\sum_{m^{\prime \prime}=1-\frac{N_{\theta}}{2}}^{\frac{N_{\theta}}{2}} W_{m^{\prime \prime}}^{m \mu} e^{i m^{\prime \prime} \theta}
$$

where $\frac{N_{\theta}}{2}=N+1$. Thus, in eq (8.33) we have included the additional term $W_{N+1}^{m \mu}$, which we are free to set equal to zero.

We can evaluate eq (8.33) at the discrete lattice points $k \Delta \theta$, where $\Delta \theta=\frac{2 \pi}{N_{\theta}}$ and $\mathrm{k}=0,1, \ldots, \mathrm{N}_{\theta}-1$. Thus, we obtain

$$
W_{m}^{\mu}(k \Delta \theta)=\sum_{m^{\prime \prime}=1-\frac{N_{\theta}}{2}}^{\frac{N_{\theta}}{2}} W_{m}^{m \mu} e^{i m^{\prime \prime} k \Delta \theta} .
$$

We now solve the set of $\mathrm{N}_{\theta}$ simultaneous equations (8.34) by making use of the orthogonality relation,

to obtain

$$
\sum_{k=0}^{N_{\theta}-1} e^{i q k \Delta \theta} \times e^{-i m^{\prime \prime} k \Delta \theta}=N_{\theta} \delta_{q m^{\prime \prime}}
$$




$$
W_{m}^{m \mu}=\frac{1}{N_{\theta}} \sum_{k=0}^{N_{\theta}-1} W_{m}^{\mu}(k \Delta \theta) e^{-i m^{\prime \prime} k \Delta \theta}
$$

where $m^{\prime \prime}=1-\frac{N_{\theta}}{2}, 2-\frac{N_{\theta}}{2}, \cdots, \frac{N_{\theta}}{2}-2, \frac{N_{\theta}}{2}-1$. Recall that $N=\frac{N_{\theta}}{2}-1$ and that $W_{m^{\prime \prime}}^{m \mu}=0$ when $m^{\prime \prime}=\frac{N_{\theta}}{2}$. From eq (8.36) we further note that

$$
W_{-m^{\prime \prime}}^{m \mu}=W_{N_{\theta}-m^{\prime \prime}}^{m \mu} .
$$

Equating eqs (8.32) and (8.36) gives the result

$$
W_{m}^{m \mu}=\frac{1}{2 \pi} \int_{0}^{2 \pi} W_{m}^{\mu}(\theta) e^{-i m^{\prime \prime} \theta} d \theta=\frac{1}{N_{\theta}} \sum_{k=0}^{N_{\theta}-1} W_{m}^{\mu}(k \Delta \theta) e^{-i m^{\prime \prime} k \Delta \theta} .
$$

Upon substituting eq (8.31) into eq (8.30), we obtain

$$
\dot{W}_{n}^{m \mu}=\sum_{m^{\prime}=-n}^{n} \Delta_{m^{\prime} m}^{n} \Delta_{m^{\prime} \mu}^{n} \sum_{m^{\prime \prime}=-N}^{N} W_{m^{\prime \prime}}^{m \mu} \int_{0}^{\pi} e^{i\left(m^{\prime \prime}-m^{\prime}\right) \theta} \sin \theta d \theta .
$$

We now have an algorithm for computing the modal-expansion coefficients $\mathrm{Q}^{\text {sma }}$ using discrete data-sampling points. Thus, from eqs (8.23) and (8.24) we obtain $W_{m}^{\mu}(k \Delta \theta)$ for $\mu= \pm 1$; $m=1-\frac{N_{\phi}}{2}, 2-\frac{N_{\phi}}{2}, \cdots, \frac{N_{\phi}}{2}-1, \frac{N_{\phi}}{2}$; and $k=0,1, \cdots, N_{\theta}-2, N_{\theta}-1$. Then, from eq (8.36) we obtain $W_{m^{\prime \prime}}^{m \mu}$ for $m^{\prime \prime}=-N,-N+1, \cdots, N-1, N$. The integral in eq (8.39) is readily evaluated analytically, so we have an algorithm for computing $\dot{W}_{n}^{m \mu}$ for $\mathrm{n}=1,2, \ldots, \mathrm{N}-1, \mathrm{~N}$ over the indicated range on $\mathrm{m}$ and $\mu$. Finally, eq (8.29) yields a linearly independent pair of equations when $\mu= \pm 1$ which can be solved for $\mathrm{Q}^{\mathrm{smn}}$.

As previously indicated, once we have calculated the modal-expansion coefficients $\mathrm{Q}^{\mathrm{smn}}$ we can substitute them back into eq (2.42) in order to compute the far-zone electric field or else we can substitute them back into eq (8.15), replacing the near-field probe coefficients $\hat{R}_{s \mu n}^{\prime}$ with farfield ideal-dipole coefficients so as to compute the coupling between the test antenna and an ideal dipole located in the far-field of the test antenna. We shall pursue this latter course in the presentation to follow.

\subsubsection{An Efficient Computational Algorithm for Computing the Far-Field Pattern from Near- Field Measurements}

\subsubsection{Computation of $\dot{W}_{n}^{m \mu}$}

In the preceding, we obtained expressions for computing the modal-expansion coefficients for the field of a radiating test antenna from near-field measurements made at discrete data sampling points over the surface of a measurement sphere. Here, we will find that it is possible 
to eliminate some duplicate computational steps in evaluating eq (8.39), which either cancel each other or else can be combined together.

We start by considering the interrelations that exist between the Fourier expansion coefficients $W_{m^{\prime \prime}}^{m \mu}$ of the measured data. On the surface of a sphere the point $(-\theta, \phi)$ is located at the same place as $(\theta, \phi+\pi)$. Now consider a probe whose dipole axis is tangent to the spherical surface at the point $(\theta, \phi)$. If we were to translate this probe by a rotation about the z-axis by $\pi$ radians to the point $(\theta, \phi+\pi)$, then the dipole axis of the probe would be pointing in the opposite direction than if the probe were translated by a rotation along a meridian of the sphere to the point $(-\theta, \phi)$. Consequently, we expect

$$
W(\phi,-\theta, \chi)=W(\phi+\pi, \theta, \chi+\pi) .
$$

We can verify this result by noting from eq (8.16) that $d_{m \mu}^{n}(-\theta)=(-)^{\mu+m} d_{m \mu}^{n}(\theta)$. Substituting this expression into eq (8.15) does indeed result in eq (8.40).

Now, from eq $(8.22 b)$ we see that eq $(8.40)$ leads to

$$
W_{m}^{\mu}(-\theta)=(-)^{\mu+m} W_{m}^{\mu}(\theta) .
$$

Instead of relying on an integral expression (8.22b) to obtain eq (8.41), we could use the discrete finite Fourier series expansions (8.23) and (8.24), along with eq (8.11), to attain that result. Now using eq (8.41) with either eq (8.32) or (8.36) leads to

Next, from eq (4.14) we see that

$$
W_{-m^{\prime \prime}}^{m \mu}=(-)^{m+\mu} W_{m^{\prime \prime}}^{m \mu}
$$

$$
\Delta_{-m^{\prime}, m}^{n} \Delta_{-m^{\prime}, \mu}^{n}=(-)^{m+\mu} \Delta_{m^{\prime} m}^{n} \Delta_{m^{\prime} \mu}^{n}
$$

Substituting eq (8.43) into eq (8.30), we obtain

$$
\begin{aligned}
\dot{W}_{n}^{m \mu} & =\Delta_{0 m}^{n} \Delta_{0 \mu}^{n} \int_{0}^{\pi} W_{m}^{\mu}(\theta) \sin \theta d \theta \\
& +\sum_{m^{\prime}=1}^{n} \Delta_{m^{\prime} m}^{n} \Delta_{m^{\prime} \mu}^{n} \int_{0}^{\pi} W_{m}^{\mu}(\theta)\left[e^{-i m^{\prime} \theta}+(-)^{m+\mu} e^{i m^{\prime} \theta}\right] \sin \theta d \theta .
\end{aligned}
$$

We now closely examine the second integral on the right in eq (8.44). Substituting the Fourier series expansion (8.31) into this integrand results in the expression

$$
\begin{aligned}
I & \equiv \int_{0}^{\pi} W_{m}^{\mu}(\theta)\left[e^{-i m^{\prime} \theta}-(-)^{m} e^{i m^{\prime} \theta}\right] \sin \theta d \theta \\
& =\sum_{m^{\prime \prime}=-N}^{N} W_{m^{\prime \prime}}^{m \mu} \int_{0}^{\pi} e^{i m^{\prime \prime} \theta}\left[e^{-i m^{\prime} \theta}-(-)^{m} e^{i m^{\prime} \theta}\right] \sin \theta d \theta,
\end{aligned}
$$


where we explicitly used the condition $\mu= \pm 1$. Let us now isolate four terms from the summation on the right side of eq (8.45), corresponding to $\left|\mathrm{m}^{\prime \prime} \pm \mathrm{m}^{\prime}\right|=1$. Expanding $\sin \theta$ into its complex exponential form and carrying out the integration, these four terms can be summed to obtain

$$
\frac{\pi}{2 i}\left[W_{m^{\prime}-1}^{m \mu}+(-)^{m} W_{-m^{\prime}+1}^{m \mu}-(-)^{m} W_{-m^{\prime}-1}^{m \mu}-W_{m^{\prime}+1}^{m \mu}\right]=0,
$$

where we have made use of eq (8.42). Accordingly, we can carry out the integration in eq (8.45) to obtain

$$
I=-\sum_{m^{\prime \prime}=-N}^{N}\left\{\frac{1+(-)^{m^{\prime \prime}-m^{\prime}}}{\left(m^{\prime \prime}-m^{\prime}\right)^{2}-1}-(-)^{m} \frac{1+(-)^{m^{\prime \prime}+m^{\prime}}}{\left(m^{\prime \prime}+m^{\prime}\right)^{2}-1}\right\} W_{m}^{m \mu},
$$

where the prime on the summation sign indicates that terms corresponding to $\left|\mathrm{m}^{\prime \prime} \pm \mathrm{m}^{\prime}\right|=1$ are excluded from the summation.

It was noticed by Larsen [31] that the summation

$$
\sum_{m^{\prime \prime}=-N}^{N} \frac{1+(-)^{m^{\prime \prime}-m^{\prime}}}{\left(m^{\prime \prime}-m^{\prime}\right)^{2}-1} W_{m}^{m \mu}
$$

has the form of a lagged-product sum and so can be summed as a $4 \mathrm{~N}$-term discrete Fourier transform using the FFT (fast Fourier transform). Here, we shall continue developing eq (8.47), with the result that we will obtain an alternative lagged-product sum which can be carried out as a 2 N-term discrete Fourier transform. Moreover, we show that halving the number of terms summed introduces no aliasing error into our formulation.

We now combine the positive and negative summation terms in eq (8.47), making use of eq (8.42), to obtain

$$
I=-\frac{1+(-)^{m^{\prime}}}{\left(m^{\prime}\right)^{2}-1}\left[1-(-)^{m}\right] W_{0}^{m \mu}-2 \sum_{m^{\prime \prime}=1}^{N}\left[\frac{1+(-)^{m^{\prime \prime}-m^{\prime}}}{\left(m^{\prime \prime}-m^{\prime}\right)^{2}-1}-(-)^{m} \frac{1+(-)^{m^{\prime \prime}+m^{\prime}}}{\left(m^{\prime \prime}+m^{\prime}\right)^{2}-1}\right] W_{m}^{m \mu}
$$

From eq (8.46) we see that the first term on the right in eq (8.48) does not appear when $\mathrm{m}^{\prime}=1$.

Now let us go back to the first integration on the right in eq (8.44). We start by noting from eqs (8.42) and (8.43) that

$$
\frac{\pi}{2 i}\left(W_{-1}^{m \mu}-W_{1}^{m \mu}\right) \Delta_{o m}^{n} \Delta_{o \mu}^{n}=0
$$

Consequently, no $W_{ \pm 1}^{m \mu}$ terms contribute to the first integration in eq (8.44). Ignoring such terms, we can substitute eq (8.31) into the first integral on the right in eq (8.44) and carry out the integration to obtain

$$
\int_{0}^{\pi} W_{m}^{\mu}(\theta) \sin \theta d \theta=2 W_{0}^{m \mu}-\sum_{m^{\prime \prime}=2}^{N} \frac{1+(-)^{m \prime}}{\left(m^{\prime \prime}\right)^{2}-1}\left[1-(-)^{m}\right] W_{m^{\prime \prime}}^{m \mu} \text {. }
$$


We are now in position to eolleet results. First, let us introduee the notation

$$
\epsilon_{k} \equiv \begin{cases}1, & k \neq 0 \\ \frac{1}{2}, & k=0\end{cases}
$$

Then, eombining eqs (8.48) and (8.49), we obtain from eq (8.44) the result

$$
\dot{\mathrm{W}}_{n}^{m \mu}=-2 \sum_{m^{\prime}=0}^{n} \epsilon_{m^{\prime}} \Delta_{m^{\prime} m}^{n} \Delta_{m^{\prime} \mu}^{n} \sum_{m^{\prime \prime}=0}^{N} \epsilon_{m^{\prime \prime}}\left[\frac{1+(-)^{m^{\prime \prime}-m^{\prime}}}{\left(m^{\prime \prime}-m^{\prime}\right)^{2}-1}-(-)^{m} \frac{1+(-)^{m^{\prime \prime}+m^{\prime}}}{\left(m^{\prime \prime}+m^{\prime}\right)^{2}-1}\right] W_{m^{\prime \prime}}^{m \mu},
$$

where the prime on the second sum in eq (8.51) indieates that terms of the form $0 / 0$ are not included in the summation.

Equation (8.51) could be used direetly for the eomputation of $W_{n}^{m \mu}$, provided that $\mathrm{N}$ is not too large. However, when $N>180$, eorresponding to $\Delta \theta$ values of less than $1^{\circ}$, it beeomes eomputationally more effieient to use the FFT to eompute the inner sum in eq (8.51). We start by rewriting eq (8.51) as

$$
\stackrel{\mathscr{W}}{n}_{n}^{m \mu}=-2 \sum_{m^{\prime}=0}^{n} \epsilon_{m^{\prime}} \Delta_{m^{\prime} m}^{n} \Delta_{m^{\prime} \mu}^{n} G_{m^{\prime}}^{m \mu}
$$

where $G_{m^{\prime}}^{m \mu}$ denotes the inner sum in eq (8.51). We break $G_{m^{\prime}}^{m \mu}$ into two parts as

$$
G_{m^{\prime}}^{m \mu}=F_{m^{\prime}}^{m \mu}-(-)^{m} H_{m^{\prime}}^{m \mu}
$$

where

$$
F_{m^{\prime}}^{m \mu}=\sum_{m^{\prime \prime}=0}^{N} \frac{1+(-)^{m^{\prime \prime}-m^{\prime}}}{\left(m^{\prime \prime}-m^{\prime}\right)^{2}-1} \epsilon_{m^{\prime \prime}} W_{m}^{m \mu}
$$

and

$$
H_{m^{\prime}}^{m \mu}=\sum_{m^{\prime \prime}=0}^{N} \frac{1+(-)^{m^{\prime \prime}+m^{\prime}}}{\left(m^{\prime \prime}+m^{\prime}\right)^{2}-1} \epsilon_{m^{\prime \prime}} W_{m^{\prime \prime}}^{m \mu} .
$$

Next, we introduce a change of notation that will enable us to more clearly foeus on the important features of the presentation. We define the set $\left\{g_{j} \mid j=0,1, \ldots, 2 N+1\right\}$, containing $N_{\theta}=2 N+2$ elements, as

$$
g_{j}=\left\{\begin{array}{cc}
\epsilon_{j} W_{j}^{m \mu}, & j=0,1, \cdots, N \\
0 & , j=N+1, N+2, \cdots, 2 N+1 .
\end{array}\right.
$$

We note from eq (8.36) that $W_{j+N_{\theta}}^{m \mu}=W_{j}^{m \mu}$; aceordingly, we can extend our definition of $\epsilon_{\mathrm{j}}$ so that we have

$$
g_{j+N_{\theta}}=g_{j}
$$

We also define the quantities 


$$
\alpha_{j}=\left\{\begin{array}{cc}
\frac{1+(-)^{j}}{j^{2}-1} & , j \neq \pm 1 \\
0 & , j= \pm 1
\end{array} .\right.
$$

Consequently, we rewrite eq (8.54) as

$$
F_{m^{\prime}}=\sum_{j=0}^{N} g_{j} \alpha_{j-m^{\prime}}, \quad H_{m^{\prime}}=\sum_{j=0}^{N} g_{j} \alpha_{j+m^{\prime}},
$$

where we have temporarily dropped the $m \mu$ superscripts. Notice that the range on the $m$ ' index in eq (8.54), or equivalently in eq (8.58), is $0 \leq \mathrm{m}^{\prime} \leq \mathrm{N}$.

We introduce a change in the indexes of summation in eq (8.58) to obtain

$$
F_{k}=\sum_{\ell=N+1-k}^{2 N+1-k} g_{\ell+k+N+1} \alpha_{\ell-N-1}, \quad H_{k}=\sum_{\ell=k}^{N+k} g_{\ell-k} \alpha_{\ell} ; \quad 0 \leq k \leq N .
$$

where we have made use of eq (8.56), recalling that $N_{\theta}=2 N+2$. Now we note from eq (8.55) that only zero terms are added to the summations in (8.59) by extending the range of the summation indexes to $0 \leq \ell \leq 2 \mathrm{~N}+1$. Thus, we have

$$
F_{k}=\sum_{\ell=0}^{2 N+1} g_{\ell+k+N+1} \alpha_{\ell-N-1}, \quad H_{k}=\sum_{\ell=0}^{2 N+1} g_{\ell-k+2 N+2} \alpha_{\ell} .
$$

Let eq (8.60) serve as a definition of $\mathrm{F}_{\mathrm{k}}$ and $\mathrm{H}_{\mathrm{k}}$ for the purpose of extending the range on $\mathrm{k}$ to $0 \leq k \leq 2 N+1$. This range extension has no effect on the first $N+1$ values of $F_{k}$ and $H_{k}$, in which we are primarily interested, but it does give us the opportunity to take the discrete Fourier transform of $\mathrm{F}_{\mathrm{k}}$ and $\mathrm{H}_{\mathrm{k}}$. Therefore,

$$
\begin{aligned}
& \sum_{k=0}^{2 N+1} F_{k} e^{i r k \Delta \theta}=\sum_{\ell=0}^{2 N+1}\left(\sum_{k=0}^{2 N+1} g_{\ell+k+N+1} e^{i r k \Delta \theta}\right) \alpha_{\ell-N-1}, \\
& \sum_{k=0}^{2 N+1} H_{k} e^{i r k \Delta \theta}=\sum_{\ell=0}^{2 N+1}\left(\sum_{k=0}^{2 N+1} g_{\ell-k+2 N+2} e^{i r k \Delta \theta}\right) \alpha_{\ell},
\end{aligned}
$$

where $0 \leq \mathrm{r} \leq 2 \mathrm{~N}+1$ and $\Delta \theta=(2 \pi) /\left(\mathrm{N}_{\theta}\right), \mathrm{N}_{\theta}=2 \mathrm{~N}+2$.

Let us examine first the inner sum in eq (8.61a). Both $e^{i r k \Delta \theta}$ and $g_{k}$ are periodic in $k$ with period $\mathrm{N}_{\theta}$, so we have

$$
\sum_{k=0}^{2 N+1} g_{\ell+k+N+1} e^{i r k \Delta \theta}=e^{i(N+1-\ell) r \Delta \theta} \sum_{k=0}^{2 N+1} g_{k} e^{i r k \Delta \theta} .
$$

Similarly, the inner sum in eq (8.61b) can be written as 


$$
\begin{aligned}
\sum_{k=0}^{2 N+1} g_{2 N+2+\ell-k} e^{i r k \Delta \theta} & =\sum_{k=0}^{\ell} g_{\ell-k} e^{i r k \Delta \theta}+\sum_{k=\ell+1}^{2 N+1} g_{2 N+2+\ell-k} e^{i r k \Delta \theta} \\
& =\sum_{k=0}^{\ell} g_{k} e^{i(\ell-k) r \Delta \theta}+\sum_{k=\ell+1}^{2 N+1} g_{k} e^{i(\ell-k) r \Delta \theta} \\
& =\sum_{k=0}^{2 N+1} g_{k} e^{i(\ell-k) r \Delta \theta}
\end{aligned}
$$

This particular result was previously obtained by Silverman [47] using a matrix analysis

formulation. Before substituting eqs (8.62) and (8.63) back into eqs (8.61), we define the discrete Fourier transform coefficients

Accordingly, eqs (8.61) become

$$
X(r)=\sum_{k=0}^{2 N+1} g_{k} e^{i r k \Delta \theta}, \quad 0 \leq r \leq 2 N+1 .
$$

$$
\begin{gathered}
\sum_{k=0}^{2 N+1} F_{k} e^{i r k \Delta \theta}=X(r) \sum_{\ell=0}^{2 N+1} \alpha_{\ell-N-1} e^{i(N+1-\ell) r \Delta \theta}, \\
\sum_{k=0}^{2 N+1} H_{k} e^{i r k \Delta \theta}=X(-r) \sum_{\ell=0}^{2 N+1} \alpha_{\ell} e^{i r \ell \Delta \theta}
\end{gathered}
$$

We now make use of the discrete Fourier transform orthogonality relation (8.35) to obtain

$$
F_{k}^{m \mu}=\frac{1}{2 N+2} \sum_{r=0}^{2 N+1} X^{m \mu}(r) B_{1}(r) e^{-i r k \Delta \theta}
$$

and

$$
H_{k}^{m \mu}=\frac{1}{2 N+2} \sum_{r=0}^{2 N+1} X^{m \mu}(-r) B_{2}(r) e^{-i r k \Delta \theta},
$$

where we have reintroduced the $m \mu$ superscripts, and where

$$
B_{1}(r)=\sum_{\ell=-N}^{N+1} \alpha_{-\ell} e^{i r \ell \Delta \theta}, \quad B_{2}(r)=\sum_{\ell=0}^{2 N+1} \alpha_{\ell} e^{i r \ell \Delta \theta} .
$$

We note from eq (8.57), incidentally, that $\alpha_{-\ell}=\alpha_{\ell}$. The $X^{m \mu}(r)$ coefficients can be obtained from eqs (8.55) and (8.64) as

$$
X^{m \mu}(r)=\sum_{m "=0}^{N} \epsilon_{m}{ }^{\prime \prime} W_{m}^{m \mu} e^{i r m " \Delta \theta}
$$

Now from eq (8.31) we obtain, with the help of eq (8.42),

$$
W_{m}^{\mu}(\theta)=\sum_{m^{\prime \prime}=0}^{N} \epsilon_{m^{\prime \prime}} W_{m}^{m \mu} e^{i m^{\prime \prime} \theta}-(-)^{m} \sum_{m^{\prime \prime}=0}^{N} \epsilon_{m^{\prime \prime}} W_{m^{\prime \prime}}^{m \mu} e^{-i m^{\prime \prime} \theta}
$$


Consequently, we have

$$
W_{m}^{\mu}(r \Delta \theta)=X^{m \mu}(r)-(-)^{m} X^{m \mu}(-r)
$$

Substituting eqs (8.66) and (8.69) into eq (8.53), we finally obtain

$$
G_{m^{\prime}}^{m \mu}=\frac{1}{2 N+2} \sum_{r=0}^{2 N+1}\left\{X^{m \mu}(r)\left[B_{1}(r)-B_{2}(r)\right]+W_{m}^{\mu}(r \Delta \theta) B_{2}(r)\right\} e^{-i r m^{\prime} \Delta \theta} .
$$

This constitutes the $2 \mathrm{~N}+2$ term discrete Fourier transform for calculating the lagged product in eq (8.51). The derivation has shown that eq (8.70) is an exact result; consequently, our formulation for calculating the lagged product will not introduce aliasing error from the FFT into the computed results.

We can make the computational task a little easier by using an $(\mathrm{N}+1)$ term FFT computation instead of a $(2 \mathrm{~N}+2)$ term FFT computation. This saving is available because we only have $N+1$ terms to sum in (8.68), and because we just need to evaluate eq (8.70) for $0 \leq \mathrm{m}^{\prime} \leq \mathrm{N}$. Thus, the computational algorithm becomes

$$
X^{m \mu}(2 p+q)=\sum_{m^{\prime \prime}=0}^{N}\left(\epsilon_{m}{ }^{\prime \prime} W_{m^{\prime \prime}}^{m \mu} e^{i q m^{\prime \prime} \Delta \theta}\right) e^{i p m^{\prime \prime} \frac{2 \pi}{N+1}} ;\left\{\begin{array}{l}
p=0,1, \cdots, N \\
q=0,1 .
\end{array}\right.
$$

and

$$
G_{m^{\prime}}^{m \mu}=\frac{1}{N_{\theta}} \sum_{q=0}^{1} e^{-i q m^{\prime} \Delta \theta} \sum_{p=0}^{N} A(2 p+q) e^{-i p m^{\prime} \frac{2 \pi}{N+1}} ; 0 \leq m^{\prime} \leq N,
$$

where

$$
A(r)=X^{m \mu}(r)\left[B_{1}(r)-B_{2}(r)\right]+W_{m}^{\mu}(r \Delta \theta) B_{2}(r) ; r=2 p+q
$$

In practice, we could precompute and store the values of $B_{1}(r)$ and $B_{2}(r)$ using

$$
\begin{aligned}
& \left.B_{1}(2 p+q)=\sum_{\ell=\frac{1-N}{2}}^{\frac{N+1}{2}}\left(\frac{2}{4 \ell^{2}-1} e^{i \ell q \frac{2 \pi}{N+1}}\right) e^{i \ell p \frac{4 \pi}{N+1}}\right) \quad p=0,1, \ldots, N \\
& \left.B_{2}(2 p+q)=\sum_{\ell=0}^{N}\left(\frac{2}{4 \ell^{2}-1} e^{i \ell q \frac{2 \pi}{N+1}}\right) e^{i \ell p \frac{4 \pi}{N+1}}\right\} q=0,1 .
\end{aligned}
$$

The computations indicated by eq (8.71) could occur immediately following the discrete Fourier transform computation eq (8.36). One would then replace the input near-field data with the computed $G_{m^{\prime}}^{m \mu}$ coefficients, using these stored coefficients later on to complete the calculation of $\dot{W}_{n}^{m \mu}$ using eq (8.52). 


\subsubsection{Completion of the Near-Field to Far-Field Computation Algorithm}

Equation (8.52) is our derived expression for computing $\stackrel{\circ}{W}_{n}^{m \mu}$ for both positive and negative values of $\mathrm{m}$ and $\mu$. However, we would like to restrict the computation of the $\Delta_{m^{\prime} m}^{n}$ coefficients to nonnegative index values, making use of eq (4.16) when the indexes are negative. We can put this on a systematic basis by modifying eqs (8.28), (8.29), and (8.52) when either $\mathrm{m}$ or $\mu$ is negative, but not both at once. Thus, when $\mathrm{m}$ and $\mu$ have opposite signs, eq (8.28) becomes

$$
W_{m n}^{\mu}=(-)^{n}\left(n+\frac{1}{2}\right) i^{m-\mu} \dot{W}_{n}^{m \mu},
$$

We retain eq (8.28) when $\mathrm{m}$ and $\mu$ have the same signs. Correspondingly, we have in place of eq $(8.52)$

$$
\stackrel{\circ}{W}_{n}^{m \mu}=-2 \sum_{m^{\prime}=0}^{n}\left(\frac{\mu m}{|m|}\right)^{m^{\prime}} \epsilon_{m^{\prime}} \Delta_{m^{\prime}|m|}^{n} \Delta_{m^{\prime} 1}^{n} G_{m^{\prime}}^{m \mu},
$$

where the quantity in parenthesis is equal to plus or minus one, depending on whether $\mathrm{m}$ and $\mu$ have the same sign or not. Thus, eqs (8.73) and (8.28) together redefine $\dot{W}_{n}^{m \mu}$, so that henceforth we will use eq (8.74) for calculating these coefficients. We also need to change eq (8.29) to correspond, resulting in

$$
\dot{W}_{n}^{m \mu}= \begin{cases}\frac{i^{\mu-m}}{n+1 / 2} \sum_{s=1}^{2} \hat{R}_{s \mu n}^{\prime} Q^{s m n} \quad, \text { when } m \& \mu \text { have the same signs } \\ (-)^{n} \frac{i^{\mu-m}}{n+1 / 2} \sum_{s=1}^{2} \hat{R}_{s \mu n}^{\prime} Q^{s m n}, \text { when } m \& \mu \text { have oppositesigns . }\end{cases}
$$

Up to this point we have talked about calculating $\stackrel{\mathscr{W}}{n}_{n}^{m \mu}$ in order to obtain the modalexpansion coefficients $\mathrm{Q}^{\mathrm{smn}}$; now we turn our attention to the problem of calculating the far-field of the test antenna, presuming that these modal-expansion coefficients are known. The most efficient method for computing the far field is to use the same Fourier series expansion coefficients for the angular-variable functions as were used to evaluate $\dot{W}_{n}^{m \mu}$. Consequently, we will use an expression similar to eq (8.15) to compute the far-field coupling between a receiving probe and the radiated field of the test antenna. The signal received by an impedance-matched receiving antenna located in the test antenna's far field is given in analogy with eq (8.17) by

$$
\begin{aligned}
\bar{W}(\phi, \theta, \chi) & =\sum_{m=1-\frac{N_{\phi}}{2}}^{\frac{N_{\phi}}{2}} \sum_{m^{\prime \prime}=-N}^{N} e^{i\left(m \phi+m^{\prime \prime} \theta\right)} \sum_{\mu=-1}^{1} e^{i \mu \chi} \\
& \times \sum_{n=\left(1,|m|,\left|m^{\prime \prime}\right|\right)}^{N} \Delta_{m^{\prime \prime} m}^{n} \Delta_{m^{\prime \prime \mu}}^{n} i^{\mu-m} \sum_{s=1}^{2} Q^{s m n} \overline{\hat{R}}_{s \mu n}^{\prime} .
\end{aligned}
$$


The prime on the $\mu$ summation sign in eq (8.76) indicates that the term $\mu=0$ is omitted. Here, the modal expansion coefficients $\mathrm{Q}^{\mathrm{smn}}$ are determined from measured data using eq (8.75), while $\overline{\hat{R}}_{s \mu n}^{\prime}$ denotes receiving coefficients, translated to the test antenna's coordinate system, of a probe located in the far field of the test antenna. The $\theta, \phi$ variables denote the angular coordinates of interest for calculating the far field, while $\chi$ is the probe rotation angle which determines a specific far-field vector component, assuming the far-field probe is an ideal dipole. Normally, the only $\chi$ values of interest are $\chi=0$ and $\chi=90^{\circ}$ (additional details concerning the equivalence between the coupling equation and the expression for the electric field when the receiver is an ideal dipole are given in Chapter VII).

Although evaluation of eq (8.76) is straightforward, it is convenient to split the calculation into separate stages for efficient handling. Thus, at the same time that $(8.75)$ is solved for a particular modal-expansion coefficient, we evaluate

$$
S_{n}^{m \mu}=i^{\mu-m} \sum_{s=1}^{2} \overline{\hat{R}}_{s \mu n}^{\prime} Q^{s m n} .
$$

The next step, in the process of calculating the coupling between the antenna under test and a receiving probe located in the far field of the test antenna, is to determine the set of Fourier transform coefficients

$$
\bar{W}_{m}^{m \mu}=\sum_{n=\left(1,|m|,\left|m^{\prime \prime}\right|\right)}^{N} \Delta_{m}^{n}{ }_{m}^{\prime \prime} \Delta_{m{ }^{\prime \prime}}^{n} S_{n}^{m \mu}
$$

Here, $\bar{W}_{m}^{m \mu}$ is the Fourier transform of the coupling between the test antenna and the far-field probe. When that probe is an ideal dipole then the inverse discrete Fourier transforms of eq (8.78) give the far-field components of the radiated electric field (for example, see eqs (7.18), (7.19) and (7.21)). These inverse descrete Fourier transforms may be expressed as

$$
\bar{W}(\phi, \theta, \chi)=\sum_{m=0}^{N_{\phi}-1} \sum_{m^{\prime \prime}=0}^{N_{\theta}-1} e^{i\left(m \phi+m^{\prime \prime} \theta\right)} \sum_{\mu=-1}^{1} e^{i \mu \chi} \bar{W}_{m^{\prime \prime}}^{m \mu} .
$$

Equation (8.79) shows the index range that needs to be considered in evaluating eq (8.78). However, from eq (8.42) we see that eq (8.78) only needs to be evaluated for $\mathrm{m}^{\prime \prime}$ index values in the range $0 \leq \mathrm{m}^{\prime \prime} \leq \mathrm{N}$, where $N=\frac{N_{\theta}}{2}-1$. The remaining $\mathrm{m}^{\prime \prime}$ coefficients required by eq (8.79) are obtained (see eq (8.37)) from

$$
\bar{W}_{N_{\theta}-m^{\prime \prime}}^{m \mu}=(-)^{m+\mu} \bar{W}_{m^{\prime \prime}}^{m \mu} \text {. }
$$

Since we wish to restrict the calculation of $\Delta_{m^{\prime \prime} m}^{n} \Delta_{m{ }^{\prime \prime}}^{n}$ to positive index values, we have, in place of eq (8.78), the expression 


$$
\bar{W}_{m}^{m \mu}=\left\{\begin{array}{c}
\sum_{n=\left(1,|m|, m^{\prime \prime}\right)}^{N} \Delta_{m^{\prime \prime},|m|}^{n} \Delta_{m^{\prime \prime}, 1}^{n} S_{n}^{m \mu}, \text { when } m \& \mu \text { have the samesigns } \\
(-)^{\prime \prime \prime} \sum_{n=\left(1,|m|, m^{\prime \prime}\right)}^{N} \Delta_{m^{\prime \prime},|m|}^{n} \Delta_{m^{\prime \prime}, 1}^{n} S_{n}^{m \mu}, \text { when } m \& \mu \text { have oppositesigns }
\end{array}\right.
$$

while eq (8.77) is changed to redefine $S_{n}^{m \mu}$ as

$$
S_{n}^{m \mu}=\left\{\begin{array}{l}
i^{\mu-m} \sum_{s=1}^{2} \overline{\hat{R}}_{s \mu n}^{\prime} Q^{s m n}, \text { when } m \& \mu \text { have the samesign } \\
(-)^{n} i^{\mu-m} \sum_{s=1}^{2} \overline{\hat{R}}_{s \mu n}^{\prime} Q^{s m n}, \text { when } m \& \mu \text { have opposite signs . }
\end{array}\right.
$$

In eq (8.81) the $\mathrm{m}^{\prime \prime}$ index is restricted to nonnegative values as a consequence of $(8.80)$.

The $\Delta_{m^{\prime}|m|}^{n} \Delta_{m^{\prime} 1}^{n}$ coefficients are computed for fixed $n$ and $m$ index values using eqs (4.15) and (4.17). Once these coefficients are generated they are used to multiply the $G_{m^{\prime}}^{m \mu}$ coefficients in eq (8.74) to compute $\dot{W}_{n}^{m \mu}$. Then the $S_{n}^{m \mu}$ coefficients are obtained using eqs (8.75) and (8.82). The partial sums, $\bar{W}_{m^{\prime \prime}}^{m \mu}(n)=(m \mu /|m|)^{m^{\prime \prime}} \Delta_{m^{\prime \prime}|m|}^{n} \Delta_{m}^{n}{ }_{m} S_{n}^{m \mu}+\bar{W}_{m^{\prime \prime}}^{m \mu}(n-1)$, are computed using the same $\Delta_{m^{\prime}|m|}^{n} \Delta_{m^{\prime} 1}^{n}$ coefficients that were used in eq (8.74). Here, $\bar{W}_{m^{\prime \prime}}^{m \mu}\left(\left[n=m^{\prime \prime}-1\right]\right)=0$ and $\bar{W}_{m}^{m \mu}(0)=0$. Once this set of computations is completed, the index $\mathrm{n}$ is incremented and the process starts over again. Thus, for any given $n$ we will have computed the partial sums

$$
\bar{W}_{m^{\prime \prime}}^{m \mu}(n) \equiv\left(\frac{m \mu}{|m|}\right)^{m^{\prime \prime}} \sum_{n^{\prime}=\left(1,|m|, m^{\prime \prime}\right)}^{n} \Delta_{m^{\prime \prime}|m|}^{n^{\prime}} \Delta_{m^{\prime \prime} 1}^{n^{\prime}} S_{n^{\prime}}^{m \mu} \text {, }
$$

thereby avoiding duplicate generation of the $\Delta_{m^{\prime}|m|}^{n} \Delta_{m^{\prime} 1}^{n}$ coefficients. In this regard the final value for eq (8.83) is $\bar{W}_{m}^{m \mu}=\bar{W}_{m}^{m \mu}(N)$. Also, except for handling the initial and final Fourier transforms involving the $\phi$ coordinate, the spherical near-field algorithm we have been presenting can be carried out on a computer using about a dozen N-term one-dimensional complex arrays while the $\mathrm{N}$-term by $\mathrm{N}$-term two-dimensional arrays containing the Fourier-transformed data could be relegated to peripheral disk storage.

Now eq (8.75) is solved for the unknown modal-expansion coefficients $Q^{\text {sman }}$, which are then used in eq (8.82) to solve for the $S_{n}^{m \mu}$ coefficients necded by cq (8.83). We can eliminate the intermediate step of solving for $\mathrm{Q}^{\mathrm{smn}}$ by obtaining $S_{n}^{m \mu}$ directly in terms of $\dot{W}_{n}^{m \beta}$, which in turn was obtained from eq (8.74). There are four cases to consider, corresponding to $\mu= \pm 1$ and $\mathrm{m}= \pm|\mathrm{m}|$. Thesc four cases are most expediently handled using matrix equation analysis. Consequently, starting from eq (8.75) we obtain 


$$
\begin{aligned}
{\left[\begin{array}{cc}
\hat{R}_{1+n}^{\prime} & \hat{R}_{2+n}^{\prime} \\
\hat{R}_{1-n}^{\prime} & \hat{R}_{2-n}^{\prime}
\end{array}\right]\left[\begin{array}{ll}
i^{-|m|} Q^{1|m| n} & i^{|m|} Q^{1-|m| n} \\
i^{-|m|} Q^{2|m| n} & i^{|m|} Q^{2-|m| n}
\end{array}\right] } \\
=\left(n+\frac{1}{2}\right)\left[\begin{array}{cc}
-i & 0 \\
0 & i
\end{array}\right]\left[\begin{array}{cc}
\dot{W}_{n}^{|m|,+} & (-)^{n} \dot{W}_{n}^{-|m|,+} \\
(-)^{n} \dot{W}_{n}^{|m|,-} & \dot{W}_{n}^{-|m|,-}
\end{array}\right]
\end{aligned}
$$

where we have used the notation $\hat{R}_{s+n}^{\prime}$ and $\hat{R}_{s-n}^{\prime}$ for $\hat{R}_{s \mu n}^{\prime}$ when $\mu= \pm 1$, and similarly $\dot{W}_{n}^{m+}$ and $\dot{W}_{n}^{m-}$ denote $\dot{W}_{n}^{m \mu}$ when $\mu= \pm 1$. Now then, we can readily solve this matrix equation for the unknown Q's, obtaining

$$
\begin{aligned}
& {\left[\begin{array}{ll}
i^{-|m|} Q^{1|m| n} & (-)^{n} i^{|m|} Q^{1-|m| n} \\
i^{-|m|} Q^{2|m| n} & (-)^{n} i^{|m|} Q^{2-|m| n}
\end{array}\right]} \\
& =\frac{n+\frac{1}{2}}{\Delta}\left[\begin{array}{cc}
-i & 0 \\
0 & i
\end{array}\right]\left[\begin{array}{ll}
\hat{R}_{2-n}^{\prime} & (-)^{n} \hat{R}_{2+n}^{\prime} \\
\hat{R}_{1-n}^{\prime} & (-)^{n} \hat{R}_{1+n}^{\prime}
\end{array}\right]\left[\begin{array}{ll}
\stackrel{\circ}{W}_{n}^{|m|,+} & \stackrel{\circ}{W}_{n}^{-|m|,+} \\
\dot{\circ}_{n}^{|m|,-} & \stackrel{\circ}{W}_{n}^{-|m|,-}
\end{array}\right],
\end{aligned}
$$

where

$$
\Delta \equiv \hat{R}_{1+n}^{\prime} \hat{R}_{2-n}^{\prime}-\hat{R}_{1-n}^{\prime} \hat{R}_{2+n}^{\prime}
$$

We now turn to the determination of the $\mathrm{S}$ coefficients in eq (8.82). We can write this equation as a matrix equation representing the four cases as follows,

$$
\begin{aligned}
{\left[\begin{array}{cc}
S_{n}^{|m|,+} & (-)^{n} S_{n}^{-|m|,+} \\
(-)^{n} S_{n}^{|m|,-} & S_{n}^{-|m|,-}
\end{array}\right] } \\
=\left[\begin{array}{cc}
i & 0 \\
0 & -i
\end{array}\right]\left[\begin{array}{ll}
\overline{\hat{R}}_{1+n}^{\prime} & \overline{\hat{R}}_{2+n}^{\prime} \\
\overline{\hat{R}}_{1-n}^{\prime} & \overline{\hat{R}}_{2-n}^{\prime}
\end{array}\right]\left[\begin{array}{ll}
i^{-|m|} Q^{1|m| n} & i^{|m|} Q^{1-|m| n} \\
i^{-|m|} Q^{2|m| n} & i^{|m|} Q^{2-|m| n}
\end{array}\right] .
\end{aligned}
$$

Let us carry out some matrix algebra with eq (8.87) to obtain the more useful form

$$
\left[\begin{array}{ll}
S_{n}^{|m|,+} & S_{n}^{-|m|,+} \\
S_{n}^{|m|,-} & S_{n}^{-|m|,-}
\end{array}\right]=\left[\begin{array}{cc}
\overline{\hat{R}}_{1+n}^{\prime} & -\overline{\hat{R}}_{2+n}^{\prime} \\
(-)^{n+1} \overline{\hat{R}}_{1-n}^{\prime} & (-)^{n} \overline{\hat{R}}_{2-n}^{\prime}
\end{array}\right]\left[\begin{array}{cc}
i & 0 \\
0 & -i
\end{array}\right]\left[\begin{array}{ll}
i^{-|m|} Q^{1|m| n} & (-)^{n} i^{|m|} Q^{1-|m| n} \\
i^{-|m|} Q^{2|m| n} & (-)^{n} i^{|m|} Q^{2-|m| n}
\end{array}\right]
$$


We recognize the $2 \times 2$ matrix on the extreme right in eq (8.88) as that given by eq (8.85). Note that eliminating the $2 \times 2$ matrix which contains the Q's results in equal treatment for $-|\mathrm{m}|$ index quantities and $+|\mathrm{m}|$ index quantities. Accordingly, we can collapse the $S$ and $W$ matrices upon inserting eq (8.85) into eq (8.88), thereby obtaining

$$
\left[\begin{array}{l}
S_{n}^{m,+} \\
S_{n}^{m,-}
\end{array}\right]=\frac{n+\frac{1}{2}}{\Delta}\left[\begin{array}{cc}
\overline{\hat{R}}_{1+n}^{\prime} & (-)^{n+1} \overline{\hat{R}}_{2+n}^{\prime} \\
(-)^{n+1} \overline{\hat{R}}_{1-n}^{\prime} & \overline{\hat{R}}_{2-n}^{\prime}
\end{array}\right]\left[\begin{array}{cc}
\hat{R}_{2-n}^{\prime} & (-)^{n} \hat{R}_{2+n}^{\prime} \\
(-)^{n} \hat{R}_{1-n}^{\prime} & \hat{R}_{1+n}^{\prime}
\end{array}\right]\left[\begin{array}{l}
\dot{W}_{n}^{m,+} \\
\dot{W}_{n}^{m,-}
\end{array}\right] \text {. }
$$

This is the general expression we have sought. We see that eqs (8.74), (8.89), and (8.81) all combine to produce the test antenna's far-field Fourier transform coefficients. The actual farfields are calculated using eq (8.79). Also, once the $S_{n}^{m \mu}$ coefficients are obtained, the modalexpansion coefficients $Q^{\text {smn }}$ of the test antenna can be obtained from the inverse of eq (8.87).

Our evaluation of eq (8.89) requires a determination of the asymptotic form of the translated receiving coefficients for an ideal-dipole probe. Thus, for an $\mathrm{x}$-directed dipole probe, substituting eq (2.33) into eq (7.18) produces the expressions

$$
{ }_{x} \overline{\hat{R}}_{1 \mu n}^{\prime}=-\frac{1}{4} \sqrt{n(n+1)} i^{-n}, \quad x_{\hat{R}_{2 \mu n}^{\prime}}^{\prime}=-\frac{\mu}{4} \sqrt{n(n+1)} i^{-n} .
$$

The case of a y-directed dipole probe is similarly obtained from eq (7.23) as

$$
y^{\overline{\hat{R}}_{1 \mu n}^{\prime}}=\frac{\mu}{4} \sqrt{n(n+1)} i^{-n+1}, \quad y_{\hat{R}_{2 \mu n}^{\prime}}^{\prime}=\frac{1}{4} \sqrt{n(n+1)} i^{-n+1} .
$$

Equations (8.90) and (8.91) differ from the asymptotic expansion of eqs (7.18) and (7.23) only by the factor $\frac{c}{k \bar{d}} e^{i k \bar{d}}$, where $\bar{d}$ asymptotically approaches infinity. This factor shall be considered understood as part of the asymptotic far-field expression.

We can now evaluate eq (8.89) for the particular case of a y-directed dipole in the far field. Substituting eq (8.91) into eq (8.89) results in

$$
\left[\begin{array}{l}
S_{n}^{m,+} \\
S_{n}^{m,-}
\end{array}\right]=i^{1-n} \sqrt{n(n+1)} \frac{n+\frac{1}{2}}{4 \Delta}\left[\begin{array}{cc}
\hat{R}_{2-n}^{\prime}-\hat{R}_{1-n}^{\prime} & (-)^{n}\left(\hat{R}_{2+n}^{\prime}-\hat{R}_{1+n}^{\prime}\right) \\
(-)^{n}\left(\hat{R}_{2-n}^{\prime}+\hat{R}_{1-n}^{\prime}\right) & \hat{R}_{2+n}^{\prime}+\hat{R}_{1+n}^{\prime}
\end{array}\right]\left[\begin{array}{l}
\dot{W}_{n}^{m_{1}+} \\
\dot{W}_{n}^{m_{,}-}
\end{array}\right] .
$$

The case of the $\mathrm{x}$-directed dipole may be obtained from this expression by multiplying the $2 \times 2$ probe coefficient matrix in eq (8.92) by the matrix, $\left|\begin{array}{cc}i & 0 \\ 0 & -i\end{array}\right|$. This result is readily secn by substituting eq (8.90) into eq (8.89) and comparing the result with eq (8.92).

To evaluate eq (8.92), the translated receiving coefficients $\hat{R}_{s \mu n}^{\prime}$ of the actual probe are needed. If we assume that a reciprocal probe is used, then the receiving coefficients $\hat{R}_{\mathrm{o \mu \nu}}^{\prime \prime}$ of the probe in its own coordinate system are given by eq (6.34) in terms of the probe's transmitting coefficients $T^{{ }^{\mu \nu}}$. It is assumed that these coefficients have bcen predetcrmincd, either 
analytically or from measured data. Once the untranslated receiving coefficients $\hat{R}_{\sigma \mu \nu}^{\prime \prime}$ are determined, the translated receiving coefficients are given by eq (5.3) as

$$
\hat{R}_{s \mu n}^{\prime}=\sum_{\sigma \nu} C_{\sigma \mu \nu}^{s n} \hat{R}_{\sigma \mu \nu}^{\prime \prime},
$$

where the $C_{o \mu \nu}^{s n}$ coefficients may be evaluated numerically using eqs (4.33) through (4.36) to calculate the $\mathrm{a}(\mu, \mathrm{n},-\mu, \nu, \mathrm{p})$ coefficients and using eq (4.38) to calculate the spherical Hankel functions. The only physical parameter that we need to evaluate the $C_{\sigma \mu v}^{s n}$ coefficients is the product $k d$ of the wave number and the measurement-sphere radius.

We conclude this treatment of probe-compensated spherical scanning with a discussion of computational savings in applying the discrete Fourier transform. We start by noting, in view of eq (8.40), that the data range as a function of the $\theta$ and $\phi$ coordinates is $0 \leq \theta \leq \pi$ and $0 \leq \phi \leq 2 \pi$. Thus, out of $\mathrm{N}_{\theta}$ data points required by the discrete Fourier transform (DFT), exactly $\left(\mathrm{N}_{\theta}\right) / 2$ sampling points suffice to specify the data along the $\theta$ coordinate. This implies that we only need to carry out half the number of $\phi$-DFT computations that would have been required if all $\mathrm{N}_{\theta}$ data measurement points were necessary.

In order to carry out the $\theta$-DFT (8.36), we rely on eq (8.41) to supply data points for angles in the range $-\pi<\theta<0$. We can cut the total $\theta$ Fourier transform processing effort in half, however, by taking advantage of the even-odd character of adjacent $m$ index values as shown by eq (8.41). Thus, for $\mathrm{m}$ even, $W_{m}^{\mu}(\theta)$ is an odd function of $\theta$, while $W_{m+1}^{\mu}(\theta)$ is an even function of $\theta$. This alternating even and odd character will permit us to simultaneously perform two $\theta$-DFT computations at a time, in view of the theorem [13] which states that, if $\mathrm{X}$ and $\mathrm{A}$ denote discrete Fourier transform pairs, then $\mathrm{X}$ is even if and only if $\mathrm{A}$ is even, and $\mathrm{X}$ is odd if and only if $\mathrm{A}$ is odd. Thus, we can take the combination of two adjacent $\phi$-Fourier transform data sets in $\theta$ as the input to the $\theta$-DFT algorithm as follows,

$$
\left.\begin{array}{rl}
X_{\ell}^{\mu}(k \Delta \theta) & =W_{2 \ell}^{\mu}(k \Delta \theta)+W_{2 \ell+1}^{\mu}(k \Delta \theta) \\
X_{\ell}^{\mu}\left(\left[N_{\theta}-k\right] \Delta \theta\right)=-W_{2 \ell}^{\mu}(k \Delta \theta)+W_{2 \ell+1}^{\mu}(k \Delta \theta)
\end{array}\right\} \begin{aligned}
& k=0,1,2, \cdots, \frac{N_{\theta}}{2} \\
& \ell=0,1,2, \cdots, \frac{N_{\phi}}{2}-1 .
\end{aligned}
$$

Equation (8.93) provides us with a definition of $X_{\ell}^{\mu}(k \Delta \theta)$ for all $\mathrm{k}$ in the range $0 \leq \mathrm{k} \leq \mathrm{N}_{\theta}$. At the points $\theta=0$ and $\theta=\pi$ the even $\mathrm{m}=2 \ell$ terms on the right side of eq (8.93) vanish, so that at these common points the above definition of $X_{l}^{\mu}(\theta)$ is consistent.

The DFT of eq (8.93) is given by

$$
A_{\ell}^{\mu}\left(m^{\prime \prime}\right)=\frac{1}{N_{\theta}} \sum_{k=0}^{N_{g}-1} X_{\ell}^{\mu}(k \Delta \theta) e^{-i m^{\prime \prime} k \Delta \theta} .
$$


Thus, $\mathrm{A}_{\ell}^{\mu}\left(\mathrm{m}^{\prime \prime}\right)$ is the sum of the Fourier transforms of even and odd $\phi$-DFT coefficients, where each Fourier transform in turn is respectively even or odd as shown by eqs (8.41) and (8.42); consequently the individual Fourier transforms can be separated from the $A_{\ell}^{\mu}\left(m^{\prime \prime}\right)$ coefficients as

$$
\left.\begin{array}{rl}
W_{m^{\prime \prime}}^{2 \ell, \mu} & =\frac{1}{2}\left[A_{\ell}^{\mu}\left(m^{\prime \prime}\right)-A_{\ell}^{\mu}\left(N_{\theta}-m^{\prime \prime}\right)\right] \\
W_{m}^{2 \ell+1, \mu} & =\frac{1}{2}\left[A_{\ell}^{\mu}\left(m^{\prime \prime}\right)+A_{\ell}^{\mu}\left(N_{\theta}-m^{\prime \prime}\right)\right]
\end{array}\right\} \begin{aligned}
& m^{\prime \prime}=0,1,2, \cdots, \frac{N_{\theta}}{2} \\
& \ell=0,1,2, \cdots, \frac{N_{\phi}}{2}-1 .
\end{aligned}
$$

The set of eqs (8.93), (8.94), and (8.95) provides us with an efficient scheme for halving the required $\theta$-DFT input data processing. The same technique can be used for processing the far-field Fourier transform coefficients, the only difference being that the Fourier transform coefficients are input to the inverse DFT processor, and the angular $\theta$ functions are output. Thus, the far-field Fourier coefficient input for the inverse DFT computation is

$$
\left.\begin{array}{c}
\bar{A}_{\ell}^{\mu}\left(m^{\prime \prime}\right)=\frac{1}{2}\left(\bar{W}_{m}^{2 \ell, \mu}+\bar{W}_{m^{\prime \prime}}^{2 \ell+1, \mu}\right) \\
\bar{A}_{\ell}^{\mu}\left(N_{\theta}-m^{\prime \prime}\right)=\frac{1}{2}\left(-\bar{W}_{m}^{2 \ell, \mu}+\bar{W}_{m^{\prime \prime}}^{2 \ell+1, \mu}\right)
\end{array}\right\} \quad \begin{aligned}
& m^{\prime \prime}=0,1,2, \cdots, \frac{N_{\theta}}{2} \\
& \ell=0,1,2, \cdots, \frac{N_{\phi}}{2}-1 .
\end{aligned}
$$

The inverse DFT is given by

$$
\bar{X}_{\ell}^{\mu}(k \Delta \theta)=\sum_{m^{\prime \prime}=0}^{N_{\theta}-1} \bar{A}_{\ell}^{\mu}\left(m^{\prime \prime}\right) e^{i m^{\prime \prime} k \Delta \theta},
$$

while the far-field pattern as a function of $\theta$ is obtained from eq (8.97) as

$$
\left.\begin{array}{l}
\bar{W}_{2 \ell}^{\mu}(k \Delta \theta)=\bar{X}_{\ell}^{\mu}(k \Delta \theta)-\bar{X}_{\ell}^{\mu}\left[\left(N_{\theta}-k\right] \Delta \theta\right) \\
\bar{W}_{2 \ell+1}^{\mu}(k \Delta \theta)=\bar{X}_{\ell}^{\mu}(k \Delta \theta)+\bar{X}_{\ell}^{\mu}\left(\left[N_{\theta}-k\right] \Delta \theta\right)
\end{array}\right\} \begin{aligned}
& k=0,1,2, \cdots, \frac{N_{\theta}}{2} \\
& \ell=0,1,2, \cdots, \frac{N_{\phi}}{2}-1 .
\end{aligned}
$$

Once the expressions (8.98) have been computed, the remaining DFT calculations that are required follow immediately from eq (8.79). Thus, the complete far-field pattern is obtained from the expression

$$
\bar{W}(\phi, \theta, \chi)=\sum_{m=0}^{N_{\phi}-1} e^{i m \phi} \sum_{\mu=-1}^{1} / \bar{W}_{m}^{\mu}(\theta) e^{i \mu \chi},
$$

where $\phi=\ell \Delta \phi, \ell=0,1, \ldots, \mathrm{N}_{\phi}-1 ; \theta=\mathrm{k} \Delta \theta, \mathrm{k}=0,1, \ldots,\left(\mathrm{N}_{\theta}\right) / 2$; and $\chi=0, \pi / 2$. 


\subsection{Recapitulation of the Spherical-Scanning Algorithm's Data-Processing Steps}

Up to this point we have developed a complete theoretical formulation for a sphericalscanning algorithm. In so doing, we have pointed out the significant expressions which are necessary for implementing the algorithm. Nevertheless, a casual reader may not find it easy to pick out these expressions from all the developmental equations used in the derivation. Accordingly, we present a recap of the data processing steps that are necessary for implementing spherical-scanning.

A precursor to near-field data processing is probe characterization. We assume the probe antenna's spherical transmitting coefficients are known, having been obtained either theoretically or from processing measured data using the spherical-scanning algorithm. The probe's receiving coefficients referred to the probe's coordinate system are then obtained from eq (6.34). From this we obtain translated-probe receiving coefficients $\hat{R}_{s \mu n}^{\prime}$ from eq (5.3), where the coefficients $C_{\sigma \mu v}^{s n}$ in eq (5.3) are given by eq (5.12) in terms of $A_{\mu \nu}^{\mu n}$ and $B_{\mu \nu}^{\mu n}$, which in turn are expressed in eqs (4.24). A recursive procedure for computing the terms in the series summations in eqs (4.24) is given in eqs (4.33) through (4.39). The implicit infinite series in eq (5.3) is suitably truncated (refer to the discussion in Chapter IX regarding truncation point selection).

The measured near field consists of two complex arrays of $\theta$-component and $\phi$-component data, where each array element corresponds to a probe-antenna measurement at equally spaced, discrete $\theta, \phi$ lattice points on the surface of a hypothetical sphere centered on the antenna under test. The spacing between contiguous lattice points is designated $\Delta \theta$ and $\Delta \phi$, where $\Delta \phi \geq \Delta \theta$, $\Delta \theta=2 \pi / N_{\theta}$, and $\Delta \phi=2 \pi / N_{\phi} . N \equiv \frac{1}{2} N_{\theta}-1$ denotes the number of spherical modes summed, and $\frac{1}{2} N_{\phi}<N$. The data arrays may be identified as corresponding to either $\chi=0$ or $\chi=\frac{\pi}{2}$ by examining the probe's transmitting coefficients to determine the probe's dominant dipole moment (refer to Chapter IX for further discussion; also see eqs (7.13) thru (7.16) for two contrasting elementary examples). Upon completing such identification, we have the two data arrays $W(\phi, \theta, \chi) ; \chi=0, \frac{\pi}{2}$.

The first step in the data processing algorithm is to carry out a $\phi$ Fourier transform, as shown by eqs (8.24a) and (8.24b). We then obtain $W_{m}^{\mu}(\theta)$ using (8.23). The $\theta$ Fourier transform is carried out as shown in eqs (8.93) thru (8.95), and then the coefficients $G_{m^{\prime}}^{m \mu}$, corresponding to a $\theta$ Fourier transform of $\left(W_{m}^{\mu}(\theta) \sin \theta\right)$, are obtained as shown by eqs (8.71) thru (8.72) (see eq (8.50) for a definition of $\epsilon_{k}$ ). The orthogonality-integration coefficients $\dot{W}_{n}^{m \mu}$, introduced by eqs (8.27) and (8.28), are obtained by carrying out the summation (8.74). The coefficients $\Delta_{m^{\prime}|m|}^{n} \Delta_{m^{\prime} 1}^{n}$ in eq (8.74) are computed at the same time that the terms in eq (8.74) are summed. 
The calculation of these coefficients is carried out using the recursion relation (4.15), with initial starting values given by eq (4.17). The far-field Fourier expansion coefficients $\bar{W}_{m^{\prime \prime}}^{m \mu}=\bar{W}_{m^{\prime \prime}}^{m \mu}(N)$ are obtained using the partial sum formula (8.83), thereby enabling the same $\Delta_{m^{\prime}|m|}^{n} \Delta_{m^{\prime} 1}^{n}$ coefficients used in eq (8.74) to be reused without having to be regenerated and without having to store these coefficients for more than one n-index value at a time. The $S_{n^{\prime}}^{m \mu}$ coefficients in eq (8.83) are obtained from the orthogonality-integration coefficients $\dot{W}_{n}^{m \mu}$ and the translated-probe receiving coefficients $\hat{R}_{s \mu n}^{\prime}$, in the case of a y-directed far-field dipole, by carrying out the matrix equation computation (8.92), where the divisor $\Delta$ in eq (8.92) is given by eq (8.86) (see the discussion following eq (8.92) for the $\mathrm{x}$-directed far-field dipole case; the far-field dipole's directional sense should agree with the probe's dominant dipole moment in order to keep the data-output array's $\theta$ and $\phi$ component identification the same as that for the data input arrays). Finally, the complete far-field pattern for the antenna under test is obtained by using the far-field Fourier expansion coefficients $\bar{W}_{m}^{m \mu}$ in the discrete Fourier transform eqs (8.96) thru (8.99).

\subsection{A Modified Spherical-Scanning Algorithm for Radiation Confined to a Single Hemisphere}

We conclude our discussion of the data processing programs by reformulating the spherical-scanning algorithm to reduce the computational effort by nearly one half when the back-hemisphere's near-field pattern for the antenna under test is essentially negligible compared to the forward-hemisphere's near-field pattern. Thus, the probe-correction spherical-scanning algorithm will be recast to simultaneously process both positive and negative $\mathrm{m}$-index data following the $\phi$-Fourier transform of the input data. These modifications will be shown to halve the computational effort when the radiation from the test antenna is confined to the forward hemisphere. That is, when any data measurements that might be made in the back hemisphere of the measurement sphere would produce essentially negligible amplitudes in comparison with those from the forward hemisphere. This condition would cut in half the data gathering effort, and with this reformulation both the computer storage requirements and the data processing effort can be halved also.

In order to avoid the excessive use of absolute value bars in this section, we here adopt the convention that the index $\mathrm{m}$ will take on only nonnegative integer values unless otherwise explicitly noted. An example of such an exception occurs when $\mathrm{m}$ is a summation index and the summation range explicitly includes negative integer values. In all other cases where a negative index $m$ is required, we shall write $-m$ to explicitly designate such a negative index. As a consequence of this convention, we replace $|\mathrm{m}|$ and $-|\mathrm{m}|$ by $\mathrm{m}$ and $-\mathrm{m}$, respectively. 
We begin our analysis of the single-hemisphere data-processing problem by not acknowledging, for the moment, that the back hemisphere can be neglected. That is, the probecorrection case modifications that we initially introduce could as readily be applied to produce a full spherical-scanning algorithm; consequently, our initial treatment will not alter either the magnitude of the data-processing effort nor the data-storage requirements, but will serve to introduce an alternative full spherical-scanning algorithm whose computational effort is readily cut in half to produce a hemispherical-scanning algorithm.

We start by forming both the sum and the difference of the row elements in the matrices on the left-hand sides of eqs (8.85) and (8.88), in each case producing two two-element column matrices, thereby retaining the same amount of information as with the original $2 \times 2$ square matrices. Thus, in place of eqs (8.88) and (8.89) we have the matrix equations

$$
\begin{aligned}
& {\left[\begin{array}{c}
\left(S_{n}^{m, 1} \pm S_{n}^{-m, 1}\right) \\
\left(S_{n}^{m,-1} \pm S_{n}^{-m,-1}\right)
\end{array}\right]=\left[\begin{array}{cc}
\overline{\hat{R}}_{1+n}^{\prime} & -\overline{\hat{R}}_{2+n}^{\prime} \\
(-)^{n+1} \overline{\hat{R}}_{1-n}^{\prime} & (-)^{n} \overline{\hat{R}}_{2-n}^{\prime}
\end{array}\right]\left[\begin{array}{cc}
i & 0 \\
0 & -i
\end{array}\right]\left[\begin{array}{l}
i^{-m}\left(Q^{1 m n} \pm(-)^{n+m} Q^{1-m n}\right) \\
i^{-m}\left(Q^{2 m n} \pm(-)^{n+m} Q^{2-m n}\right)
\end{array}\right]} \\
& =\frac{n+1 / 2}{\Delta}\left[\begin{array}{cc}
\overline{\hat{R}}_{1+n}^{\prime} & (-)^{n+1} \overline{\hat{R}}_{2+n}^{\prime} \\
(-)^{n+1} \overline{\hat{R}}_{1-n}^{\prime} & \overline{\hat{R}}_{2-n}^{\prime}
\end{array}\right]\left[\begin{array}{cc}
\hat{R}_{2-n}^{\prime} & (-)^{n} \hat{R}_{2+n}^{\prime} \\
(-)^{n} \hat{R}_{1-n}^{\prime} & \hat{R}_{1+n}^{\prime}
\end{array}\right]\left[\begin{array}{c}
\left(\dot{W}_{n}^{m, 1} \pm \dot{W}_{n}^{-m, 1}\right) \\
\left(\dot{W}_{n}^{m,-1} \pm \dot{W}_{n}^{-m,-1}\right)
\end{array}\right],
\end{aligned}
$$

where $\Delta$ is defined by eq (8.86). We will subsequently show that the sum and the difference of the $+m$ and $-m$ matrix elements in eq (8.100), as indicated by the plus-or-minus signs, are not both needed for single-hemisphere data processing.

Proceeding with the elements of the column matrix on the left side of eq (8.100), we have from eq (8.81) the expression

$$
\bar{W}_{m}^{m, \mu} \pm(-)^{m} " \bar{W}_{m}^{-m, \mu}=(\mu)^{m^{\prime \prime}} \sum_{n=\left(1, m, m^{\prime \prime}\right)}^{N} \Delta_{m^{\prime \prime}, m}^{n} \Delta_{m^{\prime \prime}, 1}^{n}\left(S_{n}^{m, \mu} \pm S_{n}^{-m, \mu}\right) \text {, }
$$

where the range on $\mathrm{m}^{\prime \prime}$ can be restricted to nonnegative integer values because of eq (8.42). The computed far field is then obtained in a manner similar to eq (8.79) using the expression

$$
\bar{W}(\phi, \theta, \chi)=\sum_{m=1-\frac{N_{\phi}}{2}}^{\frac{N_{\phi}}{2}} e^{i m \phi} \sum_{\mu=-1}^{1} e^{i \mu \chi} \bar{W}_{m}^{\mu}(\theta) \text {, }
$$

where $\bar{W}_{ \pm m}^{\mu}(\theta)$ is obtained from the two equations 


$$
\begin{gathered}
\bar{W}_{m}^{\mu}(k \Delta \theta) \pm \bar{W}_{-m}^{\mu}([N+1+k] \Delta \theta)=\sum_{m=0}^{N_{\theta}-1}\left(\bar{W}_{m^{\prime \prime}}^{m, \mu} e^{i m^{\prime \prime} k \Delta \theta} \pm \bar{W}_{m}^{-m, \mu} e^{i m^{\prime \prime}(N+1+k) \Delta \theta}\right) \\
=\sum_{m}^{N}\left(\bar{W}_{m}^{m, \mu} \pm(-)^{m \prime \prime} \bar{W}_{m}^{-m, \mu}\right) e^{i m^{\prime \prime} k \Delta \theta} .
\end{gathered}
$$

Note that one equation results from eq (8.103) by choosing the plus $(+)$ sign, while the other equation results from choosing the minus (-) sign. Also, in eq (8.103) we have used $N_{\theta}=2 N+2, \Delta \theta=\frac{2 \pi}{N_{\theta}}$, and $W_{N+1}^{m \mu}=0$.

We next evaluate the column matrix elements on the right side of eq (8.100). From eq (8.74) we obtain

$$
\dot{W}_{n}^{m, \mu} \pm \dot{W}_{n}^{-m, \mu}=-2 \sum_{m^{\prime}=0}^{n}(\mu)^{m^{\prime}} \epsilon_{m^{\prime}} \Delta_{m^{\prime} m}^{n} \Delta_{m^{\prime} 1}^{n}\left[G_{m^{\prime}}^{m, \mu} \pm(-)^{m^{\prime}} G_{m^{\prime}}^{-m, \mu}\right] \text {. }
$$

Comparing eqs (8.51) and (8.52) we see that

$$
\begin{aligned}
G_{m^{\prime}}^{m \mu} \pm(-)^{m^{\prime}} & G_{m^{\prime}}^{-m \mu}=\sum_{m^{\prime \prime}=0}^{N} \epsilon_{m^{\prime \prime}}\left[1+(-)^{m^{\prime \prime}+m^{\prime}}\right] \\
& \times\left[\frac{1}{\left(m^{\prime \prime}-m^{\prime}\right)^{2}-1}-\frac{(-)^{m}}{\left(m^{\prime \prime}+m^{\prime}\right)^{2}-1}\right]\left(W_{m^{\prime \prime}}^{m, \mu} \pm(-)^{m^{\prime \prime}} W_{m^{\prime \prime}}^{-m, \mu}\right),
\end{aligned}
$$

where we have used the fact that $(-)^{\mathrm{m}^{n}+\mathrm{m}^{\prime}}=1$ in the summation, since otherwise the summation term is zero. We can now proceed just as we did with eqs (8.53) through (8.68), defining $\hat{g}_{i}$ similar to eq (8.55) as

$$
\begin{aligned}
\hat{g}_{i} & =\epsilon_{i}\left[W_{i}^{m \mu} \pm(-)^{i} W_{i}^{-m \mu}\right] ; i & ; & =0,1, \cdots, N \\
& =0 & ; i & =N+1, N+2, \cdots, 2 N+1 .
\end{aligned}
$$

Consequently, we obtain

$$
G_{m^{\prime}}^{m \mu} \pm(-)^{m^{\prime}} G_{m^{\prime}}^{-m \mu}=\frac{1}{2 N+2} \sum_{r=0}^{2 N+1}\left(\hat{X}^{m \mu}(r) B_{1}(r)-(-)^{m} \hat{X}^{m \mu}(-r) B_{2}(r)\right) e^{-i r k \Delta \theta} \text {, }
$$

where $B_{1}(r), B_{2}(r)$ are given by eq $(8.67), \Delta \theta=\frac{\pi}{N+1}$, and

$$
\hat{X}^{m \mu}(r)=\sum_{m^{\prime \prime}=0}^{N} \epsilon_{m} \prime\left(W_{m}^{m \mu} \pm(-)^{m \prime \prime} W_{m}^{-m \mu}\right) e^{i r m " \Delta \theta} .
$$

Likewise, from eqs (8.31) and (8.42) we obtain

$$
W_{m}^{\mu}(k \Delta \theta) \pm W_{-m}^{\mu}([N+1+k] \Delta \theta)=\hat{X}^{m \mu}(r)-(-)^{m} \hat{X}^{m \mu}(-r) \text {. }
$$

Equation $(8.108)$ can now be substituted into eq (8.106) to produce a result similar to eq $(8.70)$. 
We could use the development given so far in this section to obtain the complete far-field pattern for full spherical-scanning data processing; however, this development does not introduce any advantage over the formulation already presented in the preceding section. Consequently, we now impose the assumption that the fields due to the test antenna are negligible in the reverse hemisphere of the measurement sphere. Thus, the input data is restricted to the range $0 \leq \theta<\pi / 2$, instead of to the range $0 \leq \theta \leq \pi$ which would be applicable to full-sphere nearfield scanning. The $\phi$-coordinate DFT of the input data would be carried out for each value of the $\theta$-coordinate within this new range. Then $W_{ \pm m}^{\mu}(k \Delta \theta)$, for $k=0,1,2, \cdots, \frac{N-1}{2}$, would be formulated according to eq (8.23). At this point in the data processing we would shift the $-\mathrm{m}$ index data by $180^{\circ}$ around a circle constituting a meridian of the measurement sphere. Thus, we would form $W_{-m}^{\mu}(\pi-\theta)$ in place of $W_{-m}^{\mu}(-\theta)$ for $0 \leq \theta<\pi / 2$, thereby obtaining the function $W_{-m}^{\mu}(k \Delta \theta)$ which just has nontrivial values for $\mathrm{k}$ in the range $k=\frac{N+3}{2}, \frac{N+5}{2}, \ldots, N+1$. That is, we will have created a function of argument $\pi-\theta$ which is nontrivial over the range $\pi / 2<\pi-\theta \leq \pi$. Of course, the relation (8.41) applies to this shifted function as well as it did to the original unshifted function, so the effective extended range of $W_{-m}^{\mu}(\pi-\theta)$ becomes $\frac{\pi}{2}<\pi-\theta<\frac{3 \pi}{2}$, while the effective extended range of $W_{m}^{\mu}(\theta)$ becomes $-\frac{\pi}{2}<\theta<\frac{\pi}{2}$.

With this development, for given $\mathrm{m}$ and $\mu$ indices, we have created two complementary functions, $W_{m}^{\mu}(\theta)$ and $W_{-m}^{\mu}(\pi+\theta)$, having mutually exclusive nontrivial extended ranges. We further extend the range of these two functions to the full circle $0 \leq \theta \leq 2 \pi$ by setting each function to zero over that part of the circle where the complementary function is nontrivial. Then, we can add the shifted function $W_{-m}^{\mu}(\pi+\theta)$ to the unshifted function $W_{m}^{\mu}(\theta)$ and take the discrete Fourier transform of their sum, obtaining

$$
\overline{\left(W_{m^{\prime \prime}}^{m, \mu}+(-)^{m^{\prime \prime}} W_{m^{\prime \prime}}^{-m, \mu}\right)}=\frac{1}{N_{\theta}} \sum_{k=0}^{N_{\theta}-1}\left\{W_{m}^{\mu}(k \Delta \theta)+W_{-m}^{\mu}[(k+N+1) \Delta \theta]\right\} e^{-i m^{\prime \prime} k \Delta \theta} \text {. }
$$

Recall that $\mathrm{N}_{\theta}=2 \mathrm{~N}+2$ and $\Delta \theta=2 \pi / N_{\theta}$. Note that there is no restriction on eq (8.109) that would prevent us from making use of the even-odd character of adjacent m-index coefficients to halve again the number of $\theta$-coordinate DFTs that need to be carried out. The formulation for accomplishing this is sufficiently akin to eqs (8.93) through (8.95) that we shall omit the details. Moreover, the $180^{\circ}$ data shift of the $-\mathrm{m}$ index coefficients can be carried out as an integral part of setting up the even-odd $m$ index coefficient sum and difference for FFT processing.

The overhead bar on the quantity in parenthesis in eq (8.109) serves to indicate that the two functions $W_{m}^{m, \mu}$ and $W_{m^{\prime \prime}}^{-m, \mu}$ cannot be separated. However, the combined sum can be used to obtain 


$$
\begin{aligned}
\overline{\left(G_{m^{\prime}}^{m, \mu}+(-)^{m^{\prime}} G_{m^{\prime}}^{-m, \mu}\right)} & =\frac{1}{2 N+2} \sum_{r=0}^{2 N+1}\left\{Y^{m \mu}(r)\left[B_{1}(r)-B_{2}(r)\right]\right. \\
& +\left[W_{m}^{\mu}(r \Delta \theta)+W_{-m}^{\mu}([r+N+1] \Delta \theta) B_{2}(r)\right\},
\end{aligned}
$$

where $B_{1}(r), B_{2}(r)$ were defined in eq (8.67), and

$$
Y^{m \mu}(r) \equiv \sum_{m^{\prime \prime}=0}^{N} \epsilon_{m^{\prime \prime}}\left(\overline{W_{m^{\prime \prime}}^{m, \mu}+(-)^{m^{\prime \prime}} W_{m^{\prime \prime}}^{-m, \mu}}\right) e^{i \frac{\pi}{N+1} m^{\prime \prime} r} .
$$

Once again, the solid line over the quantity in parenthesis on the left side of eq (8.110) designates that the two individual quantities $G_{m^{\prime}}^{m, \mu}$ and $G_{m^{\prime}}^{-m, \mu}$ cannot be separately resolved. Equations (8.110) and (8.111) are both computed using the FFT, so a formulation similar to eqs (8.71) and (8.72) could be applied to allow $(\mathrm{N}+1)$-term FFTs to be used to evaluate eqs $(8.110)$ and (8.111). Such a formulation is sufficiently akin to our previous results that we shall omit the details.

Next, we use eq (8.110) in an expression similar to eq (8.104) to obtain

$$
\overline{\left(\stackrel{\mathscr{W}}{n}_{n}^{m, \mu}+\stackrel{\circ}{W}_{n}^{-m, \mu}\right)}=-2 \sum_{m^{\prime}=0}^{n}(\mu)^{m^{\prime}} \epsilon_{m^{\prime}} \Delta_{m^{\prime} m}^{n} \Delta_{m^{\prime} 1}^{n} \overline{\left(G_{m^{\prime}}^{m, \mu}+(-)^{m^{\prime}} G_{m^{\prime}}^{-m, \mu}\right)} \text {. }
$$

We have again obtained a quantity whose constituent parts cannot be separated out. However, this does not prevent us from using the results of eq (8.112) in eq (8.100), one result corresponding to $\mu=+1$ and one result corresponding to $\mu=-1$, in order to obtain $\overline{\left(S_{n}^{m, \mu}+S_{n}^{-m, \mu}\right)}$. We then use this output in eq (8.101) to obtain

$$
\overline{\left(\bar{W}_{m}^{m, \mu}+(-)^{m \prime \prime} \bar{W}_{m}^{-m, \mu}\right)}=(\mu)^{m^{\prime \prime}} \sum_{n=\left(1, m, m^{\prime \prime}\right)}^{N} \Delta_{m^{\prime \prime}, m}^{n} \Delta_{m^{\prime \prime}, 1}^{n} \overline{\left(S_{n}^{m, \mu}+S_{n}^{-m, \mu}\right)} \text {. }
$$

Finally, the Fourier transform is used once again, resulting now in the separable functions

$$
\bar{W}_{m}^{\mu}(k \Delta \theta)+\bar{W}_{-m}^{\mu}([k+N+1] \Delta \theta)=\sum_{m^{\prime \prime}=0}^{N_{\theta}-1} \overline{\left(\bar{W}_{m^{\prime \prime}}^{m, \mu}+(-)^{m^{\prime \prime}} \bar{W}_{m^{\prime \prime}}^{-m, \mu}\right)} e^{i m^{\prime \prime} k \Delta \theta}
$$

We can use the even-odd character of adjacent m-index coefficients with eq (8.114) to halve the number of separate DFTs of the $\theta$-coordinate that need to be executed, using a formulation similar to that in eqs (8.96) through (8.98). We see that the $-\mathrm{m}$ index components may be identified as those Fourier coefficients in the range $\pi / 2<\theta<(3 \pi) / 2$. A second $180^{\circ}$ shift is required to bring these $-\mathrm{m}$ coefficients into proper alignment, which can be carried out at the same time that the even-odd adjacent $\mathrm{m}$ coefficients are separated in a similar fashion to eq (8.98). Then, all that remains to complete the computation of the test antenna's far field is to 
carry out the final Fourier transform (8.102). This completes our analysis of the case where the input data is essentially restricted to the to the range $0 \leq|\theta|<\pi / 2$, or to the front hemisphere of the measurement sphere.

\subsection{Conclusions}

In this chapter, we have developed an algorithm for decoupling the spherical modalexpansion coefficients, starting with the expression (5.6) for the received signal when the transmission in figure 5.1 is from left to right, and assuming the availability of measured data over a hypothetical spherical surface enclosing the test antenna. In Chapter VI (section 6.4.1), it was shown that this same algorithm can be used when the transmission direction is reversed, provided that the probe (the doubly primed antenna) is reciprocal.

We started our analysis in Section 8.2.1 with a basic theoretical development that assumes continuous coupling data over the spherical surface is available, and we outlined a computational algorithm for this case. Next, a circularly symmetric probe was introduced to eliminate the necessity of requiring a least-squares solution to solve the system of equations (8.9). Then, in Section 8.2.2, we developed a practical computational algorithm for decoupling the modal expansion coefficients $Q^{s m n}$ from discretely sampled near-field data. The number of modalexpansion coefficients that can be resolved using this algorithm is equal to half the number of discrete $\theta$-measurement points on the hypothetical spherical surface. In Section 8.2.3 we develop the theoretical basis for improving the basic measured-data algorithm that was developed earlier in section 8.2.2, thereby producing a more efficient algorithm for computing far-field antenna patterns from measured near-field data. Section 8.3 of this chapter presents a synopsis of the significant data processing steps comprising our near-field spherical-scanning algorithm. Finally, in Section 8.4 an algorithm is outlined for nearly halving the computational effort in the event that the field radiated by the test antenna is negligible in the back hemisphere of the measurement sphere.

In Chapter IX, we describe some tests that were used to validate the computer code that was developed to implement spherical scanning, and we describe an experimental verification of this program. 


\section{CHAPTER IX}

\section{NUMERICAL COMPARISONS AND SIMULATIONS FOR VALIDATING SPHERICAL-SCANNING COMPUTER CODE}

\subsection{Introduction}

Here, we deseribe a number of self-consisteney tests and data simulations that were used to validate the spherieal-scanning algorithm developed in Chapter VIII. In addition to deseribing how well the algorithm performs, we develop some interesting analytical results in the proeess of validating the computational algorithm.

The most general self-eonsisteney-test environment is obtained by using aetual measured data. Self-eonsisteney tests were carried out using data obtained from spherieal near-field measurements of a phased array 30 wavelengths in diameter. In addition to deseribing selfeonsisteney tests using aetual measured data, we diseuss results obtained by using some of the analytieal expressions from Chapter VII. This enabled us to simulate a field whose spherieal modal-expansion eoeffieients were known so that predieted results eould readily be compared against numerieal values generated by the algorithm. Also, we present a synopsis of published results presented at the 1981 Antenna Applieations Symposium [46] by a user of our algorithm, who reported that our algorithm produeed transformed far-field patterns which exhibited execllent eorrelation against measured far-field data.

Thus, using the spherieal-seanning algorithm developed in eonjunetion with this work, very good agreements have been obtained between numerieal self-eonsisteney tests, between analytical and numerical comparisons, and between measured and computed far-field patterns. More reeently, a new spherieal-seanning computer eode has been developed at the National Institute of Standards and Teehnology for use on desk-top personal computers, incorporating many of the coneepts diseussed in Chapter VIII. This new program's far-ficld output has becn extensively eompared against results obtained by transforming planar near-field data to the far-field via plane-wave speetrum analysis. Although a full deseription is outside the scope of the current diseussion, very good agreement has been obtained between the far-field patterns from these wo different algorithms [59]. 


\subsection{Numerical Self-Consistency Tests and Simulations}

Equations (8.23) and (8.24), or eq (8.14), show that the spherical-scanning algorithm processes data corresponding to the signal reccived by a probe antenna in each of two orthogonal orientations ovcr a discrete set of lattice points covering the surface of a hypothetical sphcre cnclosing the antenna under test. The algorithm's output is given by eq (8.99) as two orthogonal components of the transverse electric field at a set of lattice points covering a sphere of radius $r \rightarrow \infty$.

Instead of evaluating the output over a sphere of infinite radius, it can as readily be evaluated over a sphere of finite radius. This alternate evaluation would produce valid results so long as the "computational" sphere's radius was sufficiently large to exclude cvanescent modes. Normally, a sphere large cnough to enclose the test antenna by at least a few wavelengths would be sufficient. Of particular interest is the case when the "computational" radius is equal to $d$, the radius of the measurement sphere. In this case, the input-data sct would be recreated, less any modes of order $\mathrm{n}>\mathrm{N}$ and less any irrotational-vector components due to noise within the input data set. Morcover, a significant simplification in the computational algorithm occurs when this is done, in that all radial functions of argument kd cancel out of the formulation. This "identity transformation" case occurs when $\overline{\hat{R}}_{s \mu n}^{\prime}=\hat{R}_{s \mu n}^{\prime}$, so the matrix product in eq (8.89) divided by $\Delta$ reduces to the $2 \times 2$ identity matrix.

The application of this "identity transformation" case as a self-consistency check is straightforward. Given any arbitrary set of measured (or random) data, one pass through the computer program suffices to remove any irrotational-vector components and any modes of order $n>N$. Passing the output through a second time as a new input-data set should result in reconstruction of this second data set. Carrying this test out and comparing point-by-point the input and output data resulted in a maximum relative deviation of less than $10^{-8}$. Moreover, removing the input and output FFT proccssing from the computer program and then proceeding as beforc resulted in a maximum relative deviation of less than $10^{-12}$, whilc simply performing an FFT on an arbitrary data set followed immediatcly by an inverse FFT and comparing these two data sets resulted in a maximum rclative deviation of 1 css than $10^{-8}$. The poorer response when FFT proccssing was included was traceable to computational-accuracy limitations on the available sinc and cosine computer algorithms.

Rcturning now to the algorithm's normal configuration, let us consider what analytical changes occur when the measurement probe is defincd to be an idcal dipole. That is, wc wish to obtain an analytical cxprcssion for the $2 \times 2$ matrix product in cq (8.89) for this case. Upon 
substituting translated $\mathrm{x}$-directed dipole receiving coefficients from eq (7.18) into eq (8.92) and then multiplying by the $\left(\begin{array}{cc}i & 0 \\ 0 & -i\end{array}\right)$ matrix we obtain an expression for the case where both the far-field receiving antenna and the measurement probe are $\mathrm{x}$-directed dipoles. The result is

$$
\left[\begin{array}{c}
{ }_{x} S_{n}^{m,+} \\
{ }_{x} S_{n}^{m,-}
\end{array}\right]=\frac{n+\frac{1}{2}}{2}\left[\begin{array}{cc}
\frac{i^{-n-1}}{h_{n}^{(1)}(k d)}+\frac{i^{-n}}{\frac{1}{k d} \frac{\partial}{\partial d}\left[d h_{n}^{(1)}(k d)\right]} & (-)^{n+1}\left(\frac{i^{-n-1}}{h_{n}^{(1)}(k d)}-\frac{i^{-n}}{\frac{1}{k d} \frac{\partial}{\partial d}\left[d h_{n}^{(1)}(k d)\right]}\right) \\
(-)^{n+1}\left(\frac{i^{-n-1}}{h_{n}^{(1)}(k d)}-\frac{i^{-n}}{\frac{1}{k d} \frac{\partial}{\partial d}\left[d h_{n}^{(1)}(k d)\right]}\right) & \frac{i^{-n-1}}{h_{n}^{(1)}(k d)}+\frac{i^{-n}}{\frac{1}{k d} \frac{\partial}{\partial d}\left[d h_{n}^{(1)}(k d)\right]}
\end{array}\right]\left[\begin{array}{l}
x_{W_{n}^{m,+}} \\
{ }_{x} \dot{W}_{n}^{m_{n}-}
\end{array}\right] .
$$

The corresponding expression when both the measurement probe and the far-field receiving antenna are $y$-directed dipoles is obtained from eq (9.1) by multiplying the off-diagonal elements by -1 .

The algorithm (4.37) through (4.39) for generating spherical Hankel functions and their derivatives has been checked against numerical tables [34], so that good computational accuracy for evaluating the functions in eq (9.1) has been established. Each of the matrix elements in eq (9.1) were computed and compared against a computation of the coupling matrix (8.92) as modified for the case of an x-directed far-field dipole. The $\hat{R}_{s \mu n}^{\prime}$ coefficients in eq (8.92) were obtained by evaluating eq (5.3) using probe coefficients $\hat{R}_{\sigma \mu \nu}^{\prime \prime}$ corresponding to those of an ideal $\mathrm{x}$-directed dipole as given by eq (7.17). The result of this comparison showed excellent agrcement to approximatcly 10 significant figures, thus verifying the computational accuracy of the probe-coefficient translation algorithm for this limiting case.

The accuracy of computing the delta-delta coefficient products used in eqs (8.74) and (8.81) was checked by comparing generated values against results obtained from analytical formulas that relate the Fourier coefficients of associated Legendre functions to the Fourier coefficients defined by eq (8.16) for $d_{m 1}^{n}(\theta)$. These expressions were obtained by equating the modal-expansion formula for the field to the two-antenna coupling eq (5.6) for the special case in which the rcceiving antenna is an ideal dipole. This resulted in

$$
\begin{gathered}
2 m^{\prime} \Delta_{m^{\prime} m}^{n} \Delta_{m^{\prime} 0}^{n}=-\sqrt{n(n+1)}\left\{1+(-)^{n-m^{\prime}}\right\} \Delta_{m^{\prime} m}^{n} \Delta_{m^{\prime} 1}^{n} \\
4 m \Delta_{m^{\prime} m}^{n} \Delta_{m^{\prime} 0}^{n}=\sqrt{n(n+1)}\left\{1+(-)^{n-m^{\prime}}\right\}\left\{\Delta_{m^{\prime}+1, m}^{n} \Delta_{m^{\prime}+1,1}^{n}-\Delta_{m^{\prime}-1, m}^{n} \Delta_{m^{\prime}-1,1}^{n}\right\} .
\end{gathered}
$$

These two analytical cxpressions in eq (9.2) were successfully used in tests to validate the computational accuracy of the delta-delta coefficient generation algorithm.

A natural self-consistency check is to verify that known modal-expansion cocfficients result from using analytical formulas to gencrate input data. The desired output is readily obtained 
from eq (8.85), from which numerical results can be computed to compare against theoretical values for the modal-expansion coefficients $\mathrm{Q}^{\mathrm{smn}}$. One of the simplest tests of this kind is to generate an input-data set corresponding to an $\mathrm{x}$-directed electric-dipole source, as given by eq (7.15), and then see if the modal-expansion coefficients given by eq (7.13) for such a source are produced. This test was successfully carried out, along with a similar test using an input-data set as given by cq (7.16) for a y-directed electric-dipole source to generate modal-expansion coefficients for comparison against corresponding theoretical modal-expansion coefficients from eq (7.14). In each of these tests, the expected modal-expansion coefficients corresponding to $\mathrm{n}=1$ and $\mathrm{s}=2$ were obtained with a magnitude of 0.7071 , while all other modal-expansion coefficients that were generated had magnitudes on the order of $10^{-10}$ or less.

Using the duality principle of Maxwell's equations, the modal-expansion coefficients for an $\mathrm{x}$-directed magnetic dipole source are readily obtained, whereupon it is seen that the only nonzero coefficients for such a source are

$$
Q_{x-m a g}^{111}=-Q_{x-m a g}^{1,-1,1}=i \frac{K d s}{8 \pi} k^{2} \sqrt{2},
$$

where $\mathrm{Kds}$ is the magnetic-dipole moment. The electric-field vector on the surface of a sphere enclosing this source must be orthogonal to the electric-dipole field given by eq (7.15), again by the principal of duality and by inspection of the component fields for a dipole source [42]. Thus, another elementary-dipole test case was available, in which the program generated nontrivial $s=1$ coefficients instead of nontrivial $s=2$ coefficients.

Although the tests just dcscribed were encouraging, they were by no means exhaustive since the only nontrivial modal-expansion coefficients generated were those corresponding to $\mathrm{n}=1$. A slightly more difficult test case was available, however, using the theoretical modalexpansion coefficients given by eq (7.9) for an x-directed dipole displaced a distance $r_{0}$ along the z-axis away from the origin. The input-data set corresponding to the electric field of a translated $\mathrm{x}$-directed electric dipole can be obtained from eq (7.15) upon making the coordinate transformation,

$$
x^{\prime}=x, y^{\prime}=y, \quad z^{\prime}=z+r_{0}
$$

where the primed coordinates represent the coordinate system of the translated dipole while the unprimed coordinates represent the coordinate system in which the dipole is located at the origin of coordinates. If we make the stipulation that $d>r_{0}$, where $d=\sqrt{\left(x^{\prime}\right)^{2}+\left(y^{\prime}\right)^{2}+\left(z^{\prime}\right)^{2}}$, then the only modification that nceds to be made to eq (7.15) in order to account for dipole translation is 
to multiply each vector-field component by $\exp \left(-\mathrm{ikr}_{0} \cos \theta^{\prime}\right)$. This readily follows from the approximation for the radial distance $r$ in the unprimed coordinate system,

$$
r \equiv \sqrt{x^{2}+y^{2}+z^{2}}=\sqrt{d^{2}-2 r_{0} d \cos \theta^{\prime}+r_{0}^{2}} \approx d-r_{0} \cos \theta^{\prime} .
$$

Consequently, the zeroth-order spherical Hankel function factor, which is implicit in eq (7.15), can be written as

$$
h_{0}^{(1)}(k r)=\frac{e^{i k r}}{i k r} \approx \frac{e^{i k d}}{i k d} e^{-i k r_{0} \cos \theta^{\prime}} .
$$

Note that we assume $\theta \approx \theta^{\prime}$ for the computation of the translated-dipole fields, an approximation resulting from the stipulation $d>r_{0}$.

Translated $\mathrm{x}$-directed electric-dipole data for the case $\mathrm{r}_{0}=20 \mathrm{~cm}$ and $\mathrm{k}=1.93 \mathrm{~cm}^{-1}$ were generated and input to the computer program, resulting in computed modal-expansion coefficients which could be compared against numerical values obtained from the analytical formula (7.9). The results agreed to about 10 significant figures for values of $\mathrm{n}$ between 1 and 50; thereafter, both the coefficient magnitudes and the accuracy fell off together until, at about $\mathrm{n}=65$, all of the significant figures of accuracy in the computed coefficients had vanished, while the coefficient magnitudes were down by a factor of $10^{-10}$. This test was repeated for the $y$-directed electric dipole, using eq (7.16) times $\exp \left(-\mathrm{ikr}_{0} \cos \theta^{\prime}\right)$ for the input-data set, and the computed modal-expansion coefficients were compared against corresponding theoretical modalexpansion coefficients from eq (7.11). The results obtained in this case were comparable with results obtained for the $\mathrm{x}$-directed electric dipole case. Finally, the comparison test was repeated for the case of a probe which can be described as the sum of an elementary $x$-directed magnetic dipole and an elementary y-directed electric dipole, and once again the comparison results were comparable to the results obtained in the two previous cases. The modal-expansion coefficients corresponding to an $\mathrm{x}$-directed magnetic dipole are readily obtained using a procedure similar to that described in section 7.2 for generating electric-dipole expansion coefficients. The results for an $\mathrm{x}$-directed magnetic dipole are given by

$$
Q_{x-\text { mag. }}^{21 n}=Q_{x-\text { mag. }}^{2,-1, n}=\frac{K d s}{8 \pi} k^{2} \frac{2 n+1}{\sqrt{n(n+1)}} j_{n}\left(k r_{0}\right)
$$

and

$$
Q_{x-\text { mag. }}^{11 n}=-Q_{x-\text { mag. }}^{1,-1, n}=i \frac{K d s}{8 \pi} k^{2} \frac{2 n+1}{\sqrt{n(n+1)}} \frac{1}{k r_{0}} \frac{\partial}{\partial r_{0}}\left[r_{0} j_{n}\left(k r_{0}\right)\right]
$$


The electric-field vector for a displaced $\mathrm{x}$-directed magnetic dipole corresponding to these modalexpansion coefficients can be approximated for the primed coordinate system by the expression

$$
\underline{E}_{x-\text { mag. }} \approx-i k^{2} \frac{K d s}{4 \pi} \frac{e^{i k d}}{k d}\left(\underline{a}_{\theta^{\prime}} \sin \phi^{\prime}+\underline{a}_{\phi^{\prime}} \cos \theta^{\prime} \cos \phi^{\prime}\right) e^{-i k r_{0} \cos \theta^{\prime}} .
$$

Combining eq (9.7) with eq (7.11), we obtain the combined $x$-magnetic and y-electric displaceddipole modal-expansion coefficients chosen for the analytical comparison model

$$
\begin{aligned}
Q^{11 n} & =Q^{21 n}=-Q^{1,-1, n}=Q^{2,-1, n} \\
& =-\frac{2 n+1}{\sqrt{n(n+1)}}\left[j_{n}\left(k r_{0}\right)+\frac{i}{k r_{0}} \frac{\partial}{\partial r_{0}}\left(r_{0} j_{n}\left(k r_{0}\right)\right)\right] .
\end{aligned}
$$

The electric-field input data, corresponding to this particular combination of modal-expansion coefficients, is given by

$$
\underline{E}\left(\theta^{\prime}, \phi^{\prime}\right)=i\left(1+\cos \theta^{\prime}\right)\left[\sin \phi^{\prime} \underline{a}_{\theta^{\prime}}+\cos \phi^{\prime} \underline{a}_{\phi^{\prime}}\right] e^{-i k r_{0} \cos \theta^{\prime}} .
$$

The particular configuration of electric and magnetic dipoles used in the preceding test case were so chosen because the circular open-ended waveguide probe that was used for most of the spherical-scanning data measurements closely resembled such an elementary $\mathrm{x}$-directed magnetic and elementary $y$-directed electric dipole pair, at least insofar as the $v=1$ terms of the actual probe's modal-expansion coefficients were concerned, while higher-order $(v=2,3,4, \ldots)$ terms dropped off exponentially in magnitude. Thus, this test case produced analytical modalexpansion coefficients for comparison against an empirical model that closely resembled actual probe-coefficient data. The empirical model is discussed later in this section.

Although the computer program successfully passed the battery of tests just described, we still need to determine whether the computer program's probe-translation algorithm works correctly when the input probe-coefficient data is more complex than that for an elementarydipole probe. Fortunately, a very comprehensive test of the probe-translation algorithm was suggested by Larsen [32]. In this test, the far-fields are computed for a set of near-field input data using elementary-dipole-probe coefficients and a nominal measurement-sphere radius $\mathrm{d}$. Then, a second far-field computation is carried out for the same set of near-field input data but using for the probe a set of coefficients corresponding to an elementary dipole displaced a distance $r_{0}$ along the $z^{\prime \prime}$ axis toward the origin of the measurement sphere; that is, the probe coefficients correspond to a dipole translated along the negative $z^{\prime \prime}$ axis a distance $r_{0}$. However, this second far-field computation is carried out using a measurement-sphere radius equal to $\mathrm{d}+\mathrm{r}_{0}$; consequently, the combined action of increasing the measurement-sphere radius by $r_{0}$ 
and translating the dipole probe in the negative $z$ " direction a distance $r_{0}$ results in placing the elementary dipole itself a distance $d$ away from the measurement-sphere origin. Thus, the dipole is located at the same radial distance in both far-field computations, so with identical near-field input-data sets identical far-field outputs should result if the probe-compensated program's probe-translation algorithm is working properly.

Although the test just described was only suggested for application with an elementarydipole probe, there is no reason a more complicated probe cannot be used for the test. A translation along the negative $z^{\prime \prime}$ axis can be obtained by computing probe coefficients using a positive $z^{\prime \prime}$-axis displacement of the probe and then using the formula in section 6.4 .2 to rotate the probe's $z^{\prime \prime}$ axis by $180^{\circ}$, thereby converting from a positive $z^{\prime \prime}$-axis displacement to a negative $z$-axis displacement and at the same time orienting the direction of the $z^{\prime \prime}$ axis into rather than out of the probe. Note that the origin of the probe coordinate system is enveloped by the probe when the probe is displaced toward the origin of the measurement sphere.

Tests were carried out using the analytical formulas (7.9) and (7.13) for a translated and untranslated x-directed dipole, respectively, and using eqs (7.11) and (7.14) for a y-directed dipole. The compound $\mathrm{x}$-magnetic and y-electric dipole formulation given by eq (9.9) was also used as a test case. The near-field input data used was obtained from actual measurements of a phased array 30 wavelengths in diameter. Since theoretical probe data were used, there were no constraints on the number of probe coefficients that could be fed into the probe-translation algorithm. This contrasts with the usual situation when probe coefficients computed from experimental data are used, since even though the lower-order $v$-coefficient values are accurately measured, the higher-order probe coefficients usually have very small magnitudes that can be swamped by measurement-error noise; the resulting multiplication of these low amplitude but noisy coefficients by higher-order spherical Hankel functions, which exponentially increase in magnitude when their order exceeds their argument $\mathrm{kd}$, can result in measurement error noise swamping out the entire probe-translation algorithm output. The normal solution to this problem, which was not necessary in this particular case, is to truncate the number of probe coefficients fed into the probe-translation algorithm. However, with analytical expressions available for computing the input probe coefficients, the same number of input probe coefficients could be specified as the number of translated probe coefficients required by the algorithm (that is, $\mathrm{N}$ coefficients), even though the input probe coefficient magnitudes fell off sharply for $v$ greater than $\mathrm{kr}_{0}$. As a consequence, point-by-point comparisons of the Fourier coefficients of the 
computed far fields corresponding to translated and untranslated probes resulted in maximum relative deviations for each test case of less than $10^{-11}$.

In addition to using dipole and compound-dipole probes to test the probe translation algorithm, a highly realistic model of the actual open-ended waveguide probe used in sphericalscanning measurements was also tested. This model was based on the best analytical fit of an exponential function to the front hemisphere of measured probe data, giving the empirical probepattern model

$$
\underline{E}(\theta, \phi)=e^{-\alpha \theta^{2}}\left(\sin \phi \underline{a}_{\theta}+\cos \phi \underline{a}_{\phi}\right),
$$

where $\alpha \approx 0.85$. Obtaining a simple analytical expression for the probe coefficients corresponding to this analytical pattern did not seem feasible, so the probe coefficients were simply computed numerically using eq (9.11) as theoretical input data to the spherical-scanning program and using eq (8.85) to obtain the corresponding modal-expansion coefficients assuming an ideal-dipole receiving probe. Translated probe coefficients were likewise obtained, using as input data computed values of the expression, $\underline{\mathrm{E}}(\theta, \phi) \exp \left\{-\mathrm{ikr}_{0} \cos \theta\right\}$, where $\underline{\mathrm{E}}(\theta, \phi)$ is given by eq (9.11). The previously described probe-translation test was carried out using these computed probe coefficients, and a point-by-point comparison of the computed far fields corresponding to the translated and the untranslated probe models resulted in a relative maximum deviation of less than $10^{-5}$.

With these results, all aspects of the spherical-scanning computer program successfully passed rigorous self-consistency tests.

\subsection{Computational Aspects of Spherical-Scanning Data Processing}

\subsubsection{Computational Limitations of the Probe-Translation Algorithm}

In analogy to the successful probe-translation algorithm test just discussed, it would be of interest to consider a test where the probe is translated a distance $r_{0}$ along the positive $z^{\prime \prime}$ axis while the measurement-sphere radius is set at $d-r_{0}$. Retaining the undisplaced probe at a radius d as the comparison, we observe that this alternate comparison test is equivalent to the successful probe-translation algorithm test described at the end of the preceding section; the only difference being that the displacement distance $r_{0}$ there is simply replaced by $-r_{0}$ in the present instance. Scemingly then, we should expect as good results from this new comparison test as we had previously. However, there is a definitive analytical argument which shows that this particular comparison test will fail. 
In order to see that this new eomparison test cannot succecd, let us rccxamine some of the computational aspects of the successful probc-translation algorithm test discussed in the preeeding section. Then we ean compare our conclusions about that proccdure with corresponding aspects of the new comparison test. We start by eonsidering the joint standard of comparison in the two test eases, the coupling matrix corresponding to an elementary-dipole probe eentered at the origin of the probe's coordinate system as given by eq (9.1). Now, since spherical Hankel functions inerease exponentially in magnitude as a function of their index $\mathrm{n}$ for index values greater than their argument $\mathrm{kd}$, the coupling-matrix elements will decrease exponentially as a function of $n$ for $n>k d$, whereas for $1 \leq n \leq k d$ the coupling-matrix elements ehange very slowly in magnitude reaehing a peak value around $n=k d$. It is this general behavior that the $x$-directed coupling matrix from eq (8.92) must emulate in the case of the previously deseribed suceessful probe-translation-algorithm test where the translated-probe coefficients $\hat{R}_{s \mu n}^{\prime}$ are computed for a measurement-sphere radius $\mathrm{d}+\mathrm{r}_{0}$ and where the probe is an $\mathrm{x}$-directed dipole displaced a distanee $\mathrm{r}_{0}$ along the negative $\mathrm{z}^{\prime \prime}$-axis.

Now, from eq (7.9) we see that displaced-dipole probe coeffieients forming the input to the probe-translation algorithm remain fairly eonstant in magnitude for values of the probe index $v$ such that $1 \leq v \leq \mathrm{kr}_{0}$; thereafter, their magnitudes decrease exponcntially as a function of the index $\nu$. Consequently, if for simplicity we suppose (without loss of generality) that the sum over $v$ in eq (5.3) ean be truneated at $v=\mathrm{kr}_{0}$, and upon recalling that the sum over $\mathrm{p}$ in eq (6.10) proceeds from $\mathrm{p}=|v-\mathrm{n}|$ to $\mathrm{p}=v+\mathrm{n}$, then for values of the index $\mathrm{n}$ in the range $1 \leq \mathrm{n} \leq \mathrm{kd}$, it follows that the maximum value of the index $\mathrm{p}$ in eq (6.10) is $\mathrm{p}=\mathrm{kr}_{0}+\mathrm{kd}$. Now the argument of the spherical Hankel funetions in eq (6.10) is equal to $k\left(d+r_{0}\right)$ for the ease under discussion; consequently, this argument of the spherical Hankel functions is greater than any spherical Hankel function's index $\mathrm{p}$ for cach value of $\mathrm{n}$ in the range $1 \leq \mathrm{n} \leq \mathrm{kd}$, so that no exponentially large factor is produced by the spherical Hankel functions in eq (6.10) to be passed on to the translated-probe eoeffieicnts $\hat{R}_{s \mu n}^{\prime}$ over this range on $n$. For values of $\mathrm{n}>\mathrm{kd}$, however, the spherieal Hankel function index $p$ will exceed the argument $k\left(d+r_{0}\right)$ over at least a part of the range on this summation index, so that the $\hat{R}_{s \mu n}^{\prime}$ computed for these valucs of the index $\mathrm{n}$ will bceome exponentially large, and the ratio of $\hat{R}_{s \mu n}^{\prime}$ to $\Delta$ as given by cq (8.86) will becomc exponentially small. Now, if we comparc the dipolc-probc coupling matrix (9.1) against the x-direeted eoupling matrix from eq (8.92), using as the translated-probc eocfticients the $\hat{R}_{s \mu n}^{\prime}$ whieh we have been discussing, then we sce indeed that as a function of the index $n$ the general behavior of eorresponding matrix-element magnitudes is eonsistent between these two cases. 
This is certainly to be expected, since the numerical comparison of these two cases resulted in a maximum relative deviation of less than $10^{-11}$.

Returning now to the new comparison test where the dipole probe is displaced a distance $r_{0}$ along the positive $z^{\prime \prime}$ axis while the measurement-sphere radius is decreased to $d-r_{0}$, we find that the displaced-dipole-probe coefficients for this case are identical in magnitude with those used for the successful probe-translation-algorithm test, while the arguments of the spherical Hankel functions in eq (6.10) become $\mathrm{k}\left(\mathrm{d}-\mathrm{r}_{0}\right)$. Consequently, using the previously given reasoning we see that the exponential increase in the magnitude of the translated-probe coefficients $\hat{R}_{s \mu n}^{\prime}$ will start at an $\mathrm{n}$ value of $\mathrm{n}=\mathrm{k}\left(\mathrm{d}-\mathrm{r}_{0}\right)$. As a result, the elements of the $\mathrm{x}$-directed coupling matrix from eq (8.92) will start to decrease exponentially in magnitude at this same value of $n$, so that an equality with the dipole-probe coupling matrix (9.1) will not occur.

Although our discussion has conclusively demonstrated that the numerical computation of translated displaced-dipole-probe coefficients will fail in this particular case, this does not imply that any difficulty exists with our formulation of the problem. Indeed, we can show that a similar computational difficulty exists with the exact Graf addition-theorem formula [4, p. 101].

$$
H_{n}^{(1)}(w) e^{i n \psi}=\sum_{\nu=-\infty}^{\infty} H_{v+n}^{(1)}(Z) J_{v}(z) e^{i v \phi},|z|<|Z|,
$$

where

$$
\begin{gathered}
e^{i \psi}=\frac{\left(Z-z e^{-i \phi}\right)}{w}, \\
w=\left(z^{2}+Z^{2}-2 z Z \cos \phi\right)^{1 / 2} .
\end{gathered}
$$

From eq (9.12), we see immediately for $\phi=\pi$ and $\mathrm{z}=\mathrm{kr}, \mathrm{Z}=\mathrm{k}\left(\mathrm{d}-\mathrm{r}_{0}\right)$ that we have

$$
H_{n}^{(1)}(k d)=\sum_{v=-\infty}^{\infty}(-)^{n} J_{v}\left(k r_{0}\right) H_{v+n}^{(1)}\left(k\left[d-r_{0}\right]\right) \text {. }
$$

The same argument which showed that the numerical computation of the $\hat{R}_{s \mu n}^{\prime}$ would fail when the measurement-sphere radius is $d-r_{0}$ applies to the numerical computation of eq (9.13), even though it is an exact relation.

A suitable extension of the summation range (beyond the limits $v= \pm \mathrm{kr}_{0}$ ) combined with pairing consecutive terms to avoid roundoff error due to the Hankel function's exponential growth should enable the exact formula (9.13) to be satisfactorily used for numerical computations. However, the numerical computation of $\hat{R}_{s \mu n}^{\prime}$ using eq (5.3) requires probe coefficients $\hat{R}_{\sigma \mu \nu}^{\prime \prime}$ which are usually obtained experimentally; consequently, an extension of the summation range on $v$ in eq (5.3) beyond the limit $v=\mathrm{kr}_{0}$, where $\mathrm{r}_{0}$ is the radius of the 
minimum sphere encompassing the probe, would result in picking up high-order probe coefficients whose value has been swamped by measurement-error noise.

This suggests that for reliable computations the spherical probe-translation algorithm should be restricted to probes that have no radiating elements displaccd from the probe coordinate-system origin along the positive $z^{\prime \prime}$ axis away from the test antenna. This statement is slightly stronger than that arrived at by Larsen [32] or, in the case of cylindrical near-field scanning, by Yaghjian [60], both of whom impose the weakcr restriction,

$$
v_{\max }+n_{\max }<k d \text {. }
$$

Herc, $v_{\max }$ is the truncation point of the infinitc series over $v$ in eq $(5.3), \mathrm{n}_{\max } \equiv \mathrm{N}$, and $\mathrm{d}$ is the measurement-sphere radius or the separation distance between the probe and test-antenna coordinate-system origins. If $\mathrm{r}_{0}$ denotes the minimum-radius of a sphere just encompassing the probe and $r_{1}$ dcnotes the minimum-radius of a sphere just encompassing the test antenna, then generally [60, pp. 17-18] the modal-expansion summations for the probe and test antenna can bc terminated at $v_{\max }=\mathrm{kr}_{0}$ and at $\mathrm{n}_{\max }=\mathrm{kr}_{1}$, respectively, since beyond these limits the modalcxpansion-coefficient amplitudes of the antenna fields will decrease rapidly. Consequently, substituting these values into eq (9.14) produces the restriction

$$
r_{0}+r_{1}<d,
$$

which simply states that circumscribing spheres about the probe and test antenna shall not overlap. Note in the case of the successful probe-translation test eq $(9.15)$ reduces to $r_{1}<d$, while in the case argued to fail eq $(9.15)$ reduces to $r_{1}<d-2 r_{0}$. The minimum-radius sphere for the successful test case has a radius $r_{1}$ a little less than $r_{1} \simeq d$, but not necessarily $2 r_{0}$ less.

\subsubsection{Probe-Rotation Angles Corresponding to Measured-Data Components}

Chapter VII showed that when the receiver is an elementary dipole the two-antenna coupling cquation (5.6) reduces to the dot product between the radiatcd elcctric-ficld vector and the dipole moment. Here, we develop expressions for the modal-expansion coefficients of the radiated electric field for the case where the probe is some combination of elementary dipoles. In particular, we will represent the open-ended circular-waveguide probe which was used for most spherical-scanning data measurements by a model consisting of an x-dirceted magnetic dipolc and a y-directed electric dipolc, as given by eq (9.9). The resulting formula for the radiated field's modal expansion coefficients will then be used to discuss implications of how measured data should be processed by the spherical scanning algorithm. In the course of this devclopment we will show, for the case where the probe is a simple clementary dipole, that the modal-expansion coefficient portion of the spherical-scanning algorithm reduces to applying 
orthogonality relations between vector spherical-wave functions to the modal-expansion representation of the radiated electric field.

Before specializing to the compound probe model described above, we first present the mathematical details for the simpler case of a y-directed electric-dipole probe. Solving eq (8.26) for the test-antenna's modal-expansion coefficients, we obtain

$$
\left[\begin{array}{l}
Q^{1 m n} \\
Q^{2 m n}
\end{array}\right]=\frac{1}{\left(\hat{R}_{1+n}^{\prime} \hat{R}_{2-n}^{\prime}-\hat{R}_{2+n} \hat{R}_{1-n}^{\prime}\right)}\left[\begin{array}{cc}
\hat{R}_{2-n}^{\prime} & -\hat{R}_{2+n}^{\prime} \\
-\hat{R}_{1-n} & \hat{R}_{1+n}^{\prime}
\end{array}\right]\left[\begin{array}{c}
W_{m n}^{+} \\
W_{m n}^{-}
\end{array}\right] .
$$

The receiving coefficients for a y-directed electric dipole in its own coordinate system are given by eq (7.22), while the corresponding translated receiving coefficients are given by eq (7.23). Using the latter in eq $(9.16)$ results in

$$
\left[\begin{array}{c}
{ }_{y} Q^{1 m n} \\
{ }_{y} Q^{2 m n}
\end{array}\right]=\frac{2}{c} \frac{-i}{\sqrt{n(n+1)}}\left[\begin{array}{cc}
\frac{-i}{h_{n}^{(1)}(k d)} & \frac{i}{h_{n}^{(1)}(k d)} \\
\frac{k d}{\frac{\partial}{\partial d}\left[d h_{n}^{(1)}(k d)\right]} & \frac{k d}{\frac{\partial}{\partial d}\left[d h_{n}^{(1)}(k d)\right]}
\end{array}\right]\left[\begin{array}{c}
y^{W_{m n}^{+}} \\
W_{m n}^{-}
\end{array}\right],
$$

where the y subscripts designate that the probe is an ideal $y$-directed dipole. We continue our development by substituting the integral expression (8.27) for $W_{m n}^{\mu}$ into eq (9.17) and using eq (8.23) for $W_{m}^{\mu}(\theta)$ and eq (7.20) for $d_{m \mu}^{n}(\theta)$ in the integrand of eq (8.27). After simplifying, we obtain

$$
\left[\begin{array}{l}
{ }_{y} Q^{1 m n} \\
{ }_{y} Q^{2 m n}
\end{array}\right]=\frac{1}{c} \frac{2 n+1}{n(n+1)}\left[\begin{array}{c}
\frac{1}{h_{n}^{(1)}(k d)} \int_{0}^{\pi}\left[{ }_{y} W_{m}^{0}(\theta) \frac{\partial d_{m o}^{n}(\theta)}{\partial \theta}-{ }_{y} W_{m}^{\frac{\pi}{2}}(\theta) \frac{i m d_{m o}^{n}(\theta)}{\sin \theta}\right] \sin \theta d \theta \\
\frac{k d}{\frac{\partial}{\partial d}\left[d h_{n}^{(1)}(k d)\right]} \int_{0}^{\pi}\left[{ }_{y} W_{m}^{\frac{\pi}{2}}(\theta) \frac{\partial d_{m o}^{n}(\theta)}{\partial \theta}+{ }_{y} W_{m}^{0}(\theta) \frac{i m d_{m o}^{n}(\theta)}{\sin \theta}\right] \sin \theta d \theta
\end{array}\right] .
$$

This matrix equation can be simplified by recalling eqs (2.31) and (2.32) for the transverse vector spherical-wave functions and eq (2.41) for the spherical Hankel functions. First, let us define

$$
\underline{G}_{n}^{s m}(\theta)=\underline{G}_{n}^{s m}(\theta, \phi) e^{-i m \phi} .
$$

Consequently, eq (9.18) can be simplified to obtain

$$
{ }_{y} Q^{s m n} R_{s n}^{(1)}(d)=\frac{1}{c} \frac{2 n+1}{n(n+1)} \int_{0}^{\pi} \underline{G}_{n}^{s m *}(\theta) \cdot\left[{ }_{y} W_{m}^{\frac{\pi}{2}}(\theta) \underline{a}_{\theta}-{ }_{y} W_{m}^{0}(\theta) \underline{a}_{\phi}\right] \sin \theta d \theta .
$$


For contrast with these results, the expression equivalent to eq (9.18) for an $\mathrm{x}$-directed electric dipole is given by

$$
\left[\begin{array}{l}
{ }_{x} Q^{1 m n} \\
{ }_{x} Q^{2 m n}
\end{array}\right]=\frac{1}{c} \frac{2 n+1}{n(n+1)}\left[\begin{array}{c}
\frac{1}{h_{n}^{(1)}(k d)} \int_{0}^{\pi}\left[\frac{-i m d_{m o}^{n}(\theta)}{\sin \theta}{ }_{x} W_{m}^{0}(\theta)-\frac{\partial d_{m o}^{n}(\theta)}{\partial \theta}{ }_{x} W^{\frac{\pi}{2}}(\theta)\right] \sin \theta d \theta \\
\frac{k d}{\frac{\partial}{\partial d}\left[d h_{n}^{(1)}(k d)\right]} \int_{0}^{\pi}\left[\frac{\partial d_{m o}^{n}(\theta)}{\partial \theta}{ }_{x} W_{m}^{0}(\theta)-\frac{i m d_{m o}^{n}(\theta)}{\sin \theta}{ }_{x} W_{m}^{\frac{\pi}{2}}(\theta)\right] \sin \theta d \theta
\end{array}\right],
$$

which simplifies, upon introducing the transverse vector spherical-wave function expressions

$$
\begin{aligned}
& { }_{x} Q^{s m n} R_{s n}^{(1)}(d)=\frac{1}{c} \frac{2 n+1}{n(n+1)} \int_{0}^{\pi} G_{n}^{s m *}(\theta) \cdot\left[{ }_{x} W_{m}^{0}(\theta) \underline{a}_{\theta}+{ }_{x} W_{m}^{\frac{\pi}{2}}(\theta) \underline{a}_{\phi}\right] \sin \theta d \theta .
\end{aligned}
$$

Now from eqs $(2.42),(7.26)$ and $(7.27)$ we see that with a y-directed electric-dipole probe we obtain the radiated electric field's $\theta$ component when $\chi=\pi / 2$ and the $\phi$ component when $\chi=\pi$, while with a $\mathrm{x}$-directed electric-dipole probe we obtain the radiated electric field's $\theta$ component when $\chi=0$ and the $\phi$ component when $\chi=\pi / 2$. Vectorially combining the expressions for the signal received by either elementary-dipole probe, we obtain

$$
\underline{W}(\theta, \phi) \equiv \frac{c}{2} \sum_{s n m} Q^{s m n} R_{s n}^{(1)}(d) \underline{G}_{n}^{s m}(\theta, \phi),
$$

where $\underline{W}(\theta, \phi)$ represents a vector combination of the signals received by an ideal-dipole probe when it is respectively aligned along the $\theta$ and $\phi$ coordinate axes (see eq (7.21) or eq (7.25)). Now we can apply the orthogonality relation (2.39) to eq (9.23) to obtain

$$
\frac{1}{c} \frac{2 n+1}{n(n+1)} \int_{0}^{\pi} \underline{W}_{m}(\theta) \cdot \underline{G}_{n}^{s m *}(\theta) \sin \theta d \theta=Q^{s m n} R_{s n}^{(1)}(d)
$$

where (compare to eq $(8.19))$

$$
\underline{W}_{m}(\theta)=\frac{1}{2 \pi} \int_{0}^{2 \pi} \underline{W}(\theta, \phi) e^{-i m \phi} d \phi .
$$

Consequently, comparing eqs (9.22) and (9.24), we see in analogy to eq (7.21) that

$$
W_{x}(\phi, \theta, \chi=0) \underline{a}_{\theta}+W_{x}\left(\phi, \theta, \chi=\frac{\pi}{2}\right) \underline{a}_{\phi}=\underline{W}(\theta, \phi),
$$

whereas, comparing eqs (9.20) and (9.24) results in an expression analogous to eq (7.25)

$$
W_{y}\left(\phi, \theta, \chi=\frac{\pi}{2}\right) \underline{a}_{\theta}-W_{y}(\phi, \theta, \chi=0) \underline{a}_{\phi}=\underline{W}(\theta, \phi) \text {. }
$$

Comparing eqs (7.26) with eqs $(9.26)$, we find that the vector-component $\chi$-angle identification for the radiated electric field corresponds to the ideal-dipole probe's vector componcnt $\chi$-angle identification. Moreover, when the probe is an ideal dipole, eqs (9.24) and (9.25) show that the 
integral expressions developed in Chapter VIII for obtaining the radiated electric field's modalexpansion coefficients are equivalent to applying the vector spherical-wave function orthogonality relation (2.39) to the radiated electric field.

At this point, we are ready to return to our previously described model for an open-ended circular-waveguide probe. The nonzero receiving coefficients for a combined $\mathrm{x}$-directed magnetic-dipole and a y-directed electric-dipole model for the probe are given by

$$
\hat{R}_{1,1,1}^{\prime \prime}=-\hat{R}_{1,-1,1}^{\prime \prime}=\hat{R}_{2,1,1}^{\prime \prime}=\hat{R}_{2,-1,1}^{\prime \prime}=i \frac{c}{3 \sqrt{2}} .
$$

Using eqs (4.31), (4.32), and (5.12) in eq (5.3) with these probe coefficients, we obtain the translated-probe receiving coefficients corresponding to this model,

$$
\begin{aligned}
\hat{R}_{1,1, n}^{\prime} & =-\hat{R}_{1,-1, n}^{\prime}=\hat{R}_{2,1, n}^{\prime}=\hat{R}_{2,-1, n}^{\prime} \\
& =\frac{i c}{4} \sqrt{n(n+1)}\left\{\frac{1}{k d} \frac{\partial}{\partial d}\left[d h_{n}^{(1)}(k d)\right]+i h_{n}^{(1)}(k d)\right\} .
\end{aligned}
$$

Substituting these coefficients into eq (9.16) and using our previously described procedure for obtaining an expression for the modal-expansion coefficients results in

$$
Q^{s m n}=\frac{1}{c} \frac{(2 n+1) i^{1-s}}{n(n+1)\left\{R_{1 n}^{(1)}(d)-i R_{2 n}^{(1)}(d)\right\}} \int_{0}^{\pi} G_{n}^{s m *}(\theta) \cdot\left[W_{m}^{\frac{\pi}{2}}(\theta) a_{\theta}-W_{m}^{0}(\theta) \underline{a}_{\phi}\right] \sin \theta d \theta \text {. }
$$

Let us consider the implications of this result when actual probe-coefficients for an openended circular-waveguide probe are used with the probe-correction computer program. By selecting $\mathrm{W}(\phi, \theta, \chi=0)$ as the negative of the measured $\phi$-component and $\mathrm{W}\left(\phi, \theta, \chi=\frac{\pi}{2}\right)$ as the measured $\theta$-component, we should obtain more accurately computed far fields than with any other component choice. Using probe coefficients obtained from measured probe data, we ran a test to determine the sensitivity of the computed far-fields to the number of $\nu$-values of the $\hat{R}_{\sigma \mu \nu}^{\prime \prime}$ probe coefficients used by the computer program. The computed far-field output was found to be moderately sensitive to early truncation of the sum on $v$ in eq (5.3). Then, we ran another test in which the measured $\theta$-component data was fed into the computer program as $\mathrm{W}(\phi, \theta, \chi=0)$ while the measured $\phi$-componcnt data was fed into the computer program as $\mathrm{W}\left(\phi, \theta, \chi=\frac{\pi}{2}\right)$. This second case resulted in shallower far-field nulls and insensitivity to early truncation of the sum on $v$ in eq (5.3), thercby confirming that the first choice was superior.

\subsection{Experimental Verification}

In this section we shall recount particulars of an experimental verification which used the spherical near-field program developed in conjunction with this work for the data processing. 
The material presented here is extracted from a paper presented by Shashi Sanzgiri at the 1981 Antenna Applications Symposium [46].

An open array consisting of $10 \times 35$ dipole elements incorporating an L-band feed network was used in this test. Sum, difference, and omni (omnidirectional) azimuth-plane patterns were gencrated by the feed network, while the elevation-plane patterns for all three channels were similarly shaped beams. The sum and difference (delta) patterns were typical monopulse low sidelobe, narrow beam patterns $\left(2.5^{\circ} \mathrm{HPBW}\right)$ in azimuth. The omni channel, utilized for sidelobe suppression (SLS), had omnidirectional azimuth-planc coveragc. The authors felt that this antenna's low sidelobes, backlobes, shaped beams, and omnidirectional characteristics would provide a good test of the practical feasibility of spherical near-field scanning. Far-field pattern measurements of the test antenna were taken for comparison against the transformed spherical ncar-field to far-field data. The probe antenna for the spherical near-field measurements was mounted dircctly on the tower with the antenna under test. As a consequence, the measurement sphere radius was nearly equal to the radius of a minimum sphere that just enclosed the test antenna. The probe was an open-ended cylindrical waveguide with an orthomode transducer for resolving the incident radiation into orthogonal components. The theoretical far-field pattern of the probe was used to generate modal-expansion coefficients for the probe-correction algorithm.

The near field was sampled at $1^{\circ}$ intervals in both elevation and azimuth while the antenna was rotated in azimuth and stepped in elevation. One port of the orthomode transducer was sampled during forward scan while the other port was sampled during reverse scan. Tie scans were taken to compensate for receiver drift and temperature variation, as the data-acquisition time was ncarly 5 hours per channel.

The test setup and pertinent data-acquisition parameters are reproduced ${ }^{1}$ from [46] in figures 9-1 and 9-2. Perspective plots of the measured near-field's and computed far-field's principal components are shown in figures 9-3 and 9-4, respectively. ${ }^{1}$ Figures 9-5 through 9-8 show the correlation between measured and computed far-field patterns for the three channels. ${ }^{1}$ The authors indicated in their paper [46] that the correlations obtained between computcd and measured far-field patterns went beyond their expectations in view of the short near-field measurement-sphere radius and the different reflection environments between the near-field and far-field measurements.

${ }^{1}$ Figures 9-1 through 9-8 are reproduced from [46] with the author's permission. 
OPEN ARRAY SNFP

IEST PARAMETERS

$\begin{array}{ll}\text { ANTENNA } & 5^{\prime} \times 26^{\prime} \text { PLANAR ARRAY } \\ \text { POSITIONER } & \text { AZ/EL, POL SCANS } \\ \text { FREQUENCY } & 1.06 \mathrm{GHZ} \\ \text { D/ } \lambda \text { RATIO } & 33.6 \\ \text { NF RANGE LENGTH } & 15.6^{\prime} \\ \text { CHAINELS } & \text { SUM, DIFFERENCE \& OMNI } \\ \text { SAMPLING INTERVAL } & 1^{\circ} \text { IN AZ AND EL } \\ \text { SCAN COVERAGE } & 0^{\circ} \leq \theta \leq 130^{\circ} \\ \text { ROTATION SPEED } & 7^{\circ} / \text { SEC } \\ \text { PROBE } & \text { DUAL POLARIZED CYLINDRICAL, } \\ & \text { OPEN ENDED WAVEGUIDE }\end{array}$

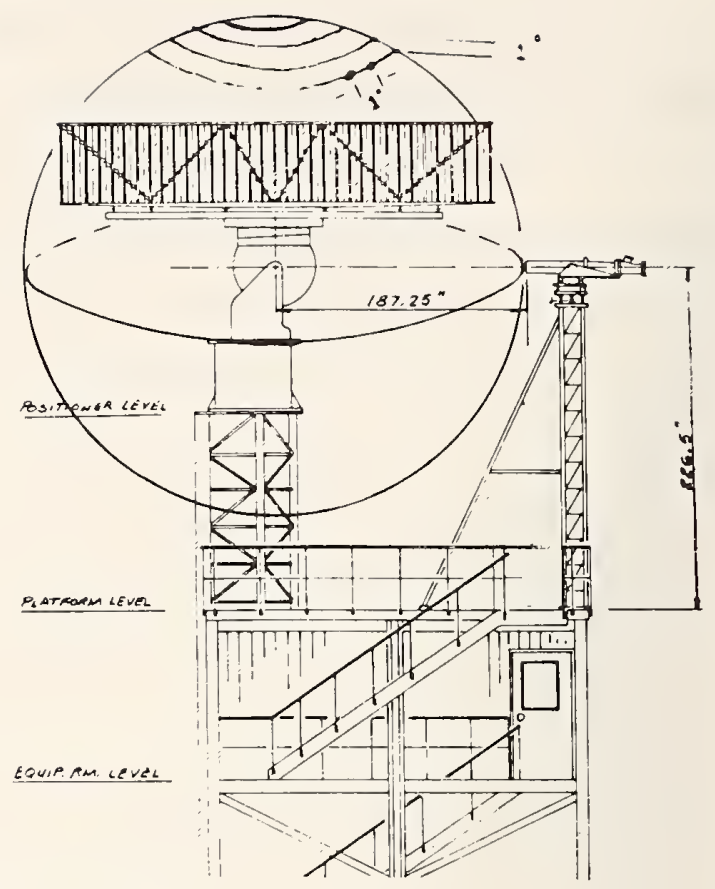

Figure 9-1. Test Parameters

figure reproduced from [46] by permission

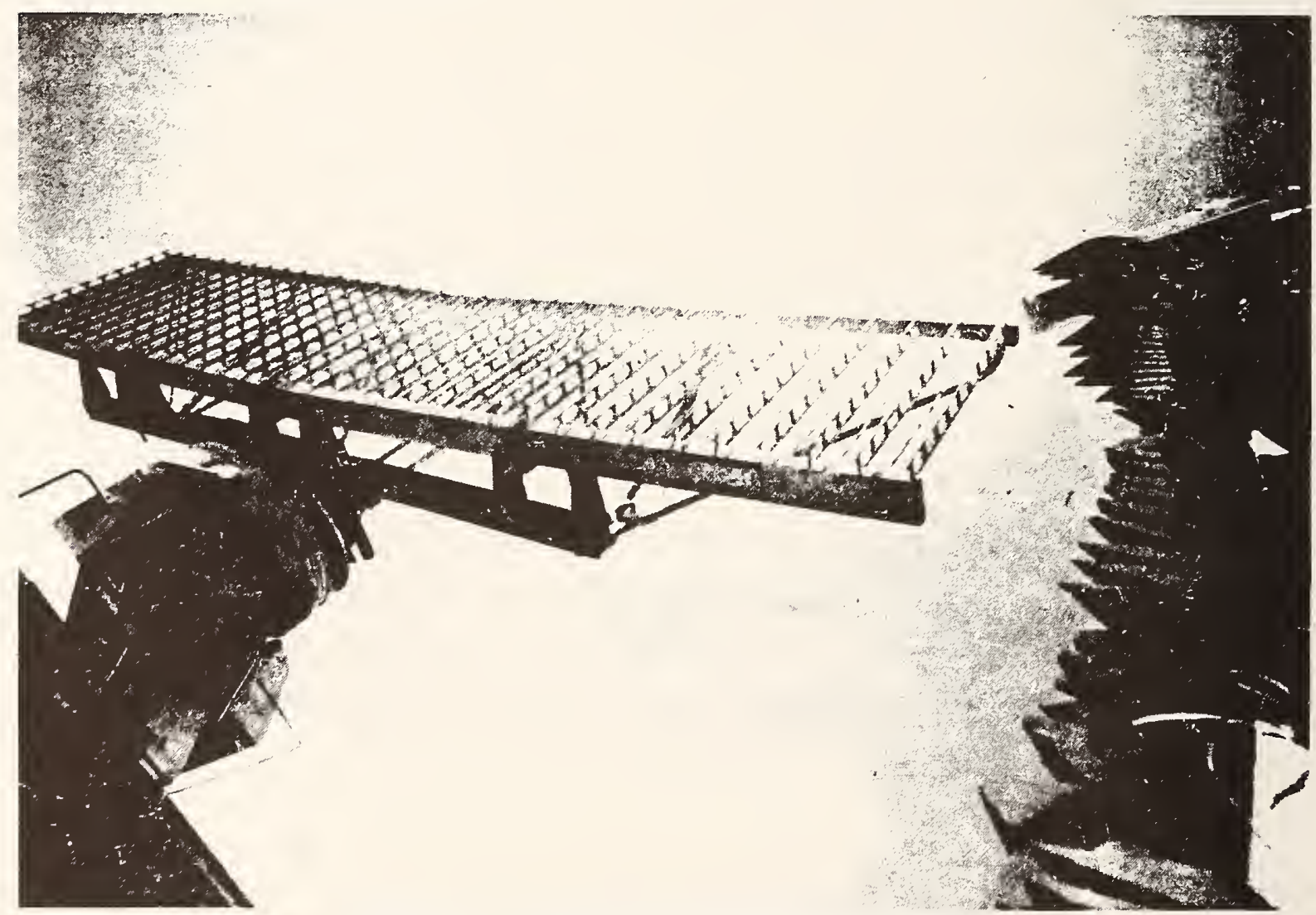

Figure 9.2. Open-array near-field test setup. 

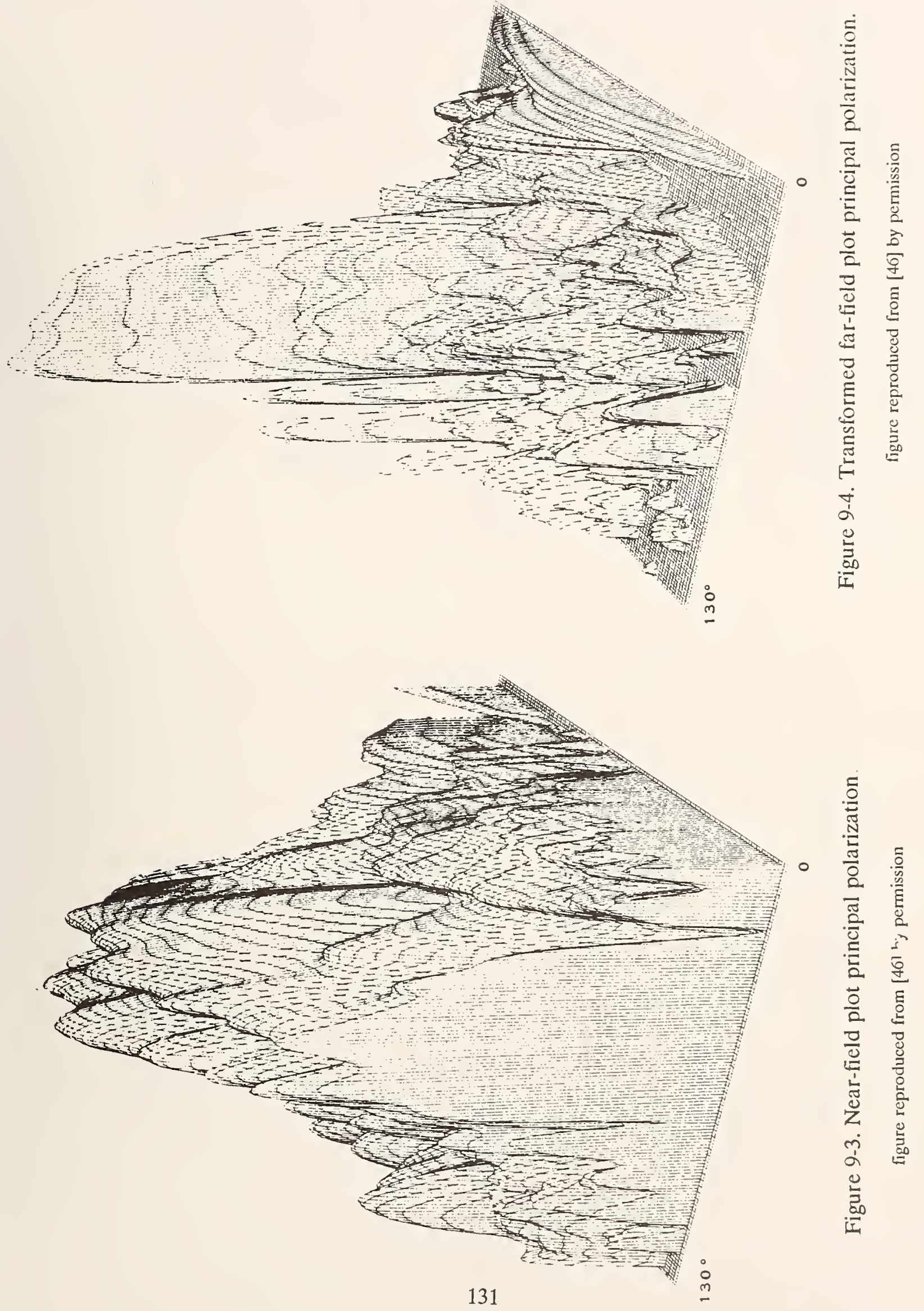
figure reproduced from [46] by permission

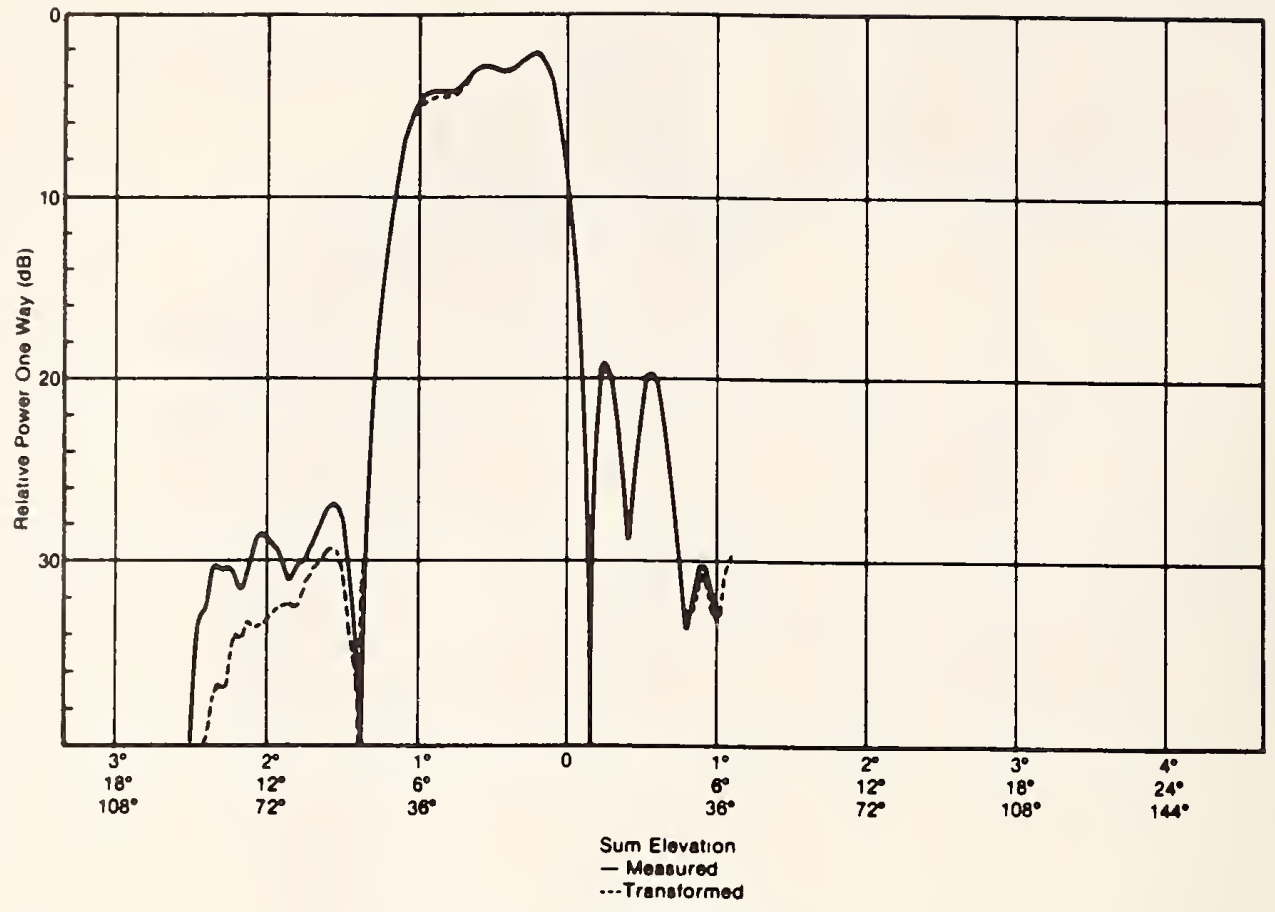

Figure 9-5. Sum elevation.

figure reproduced from [46] by permission

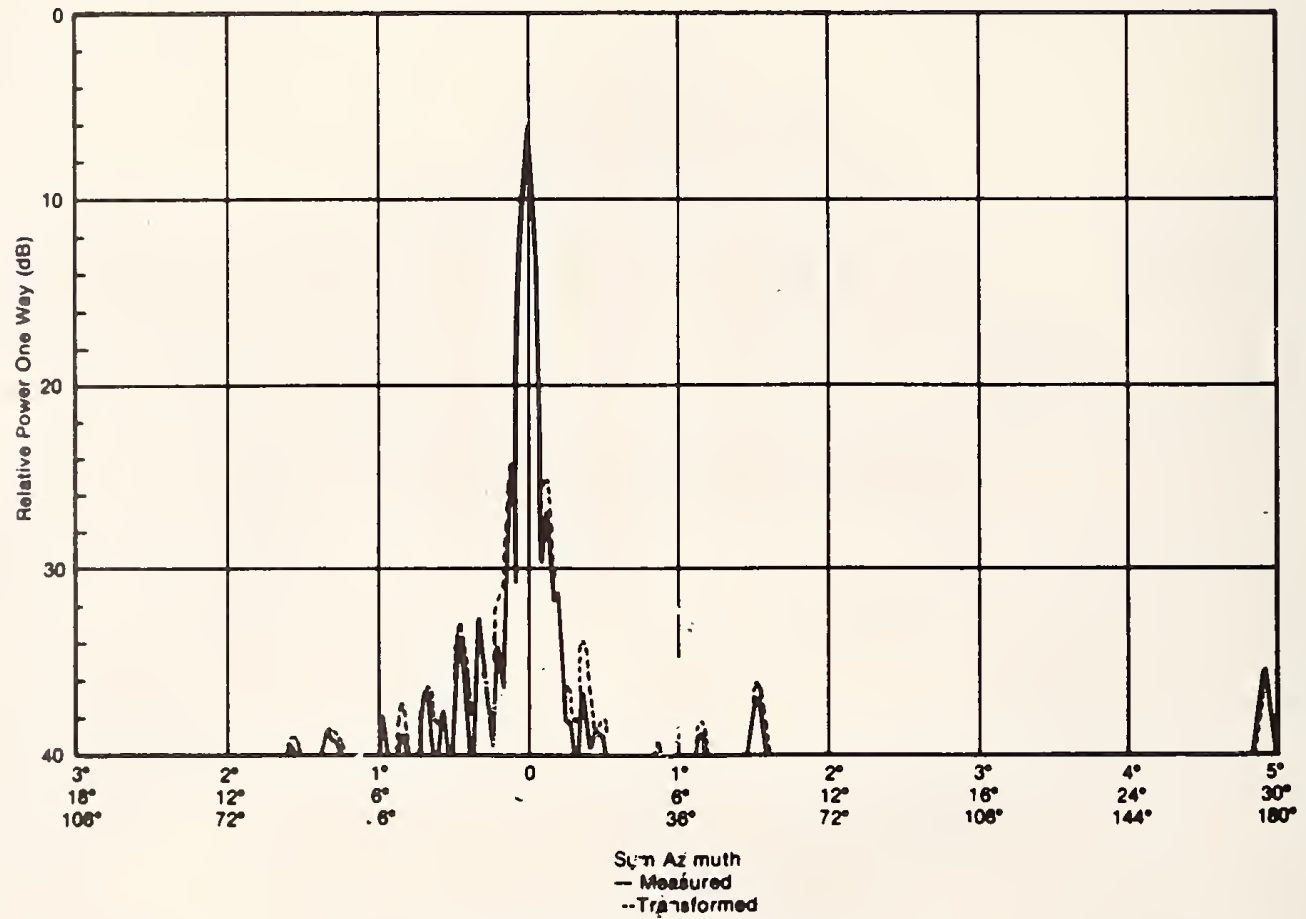

Figure 9.6. Sum azimuth 
figure reproduced from [46] by permission

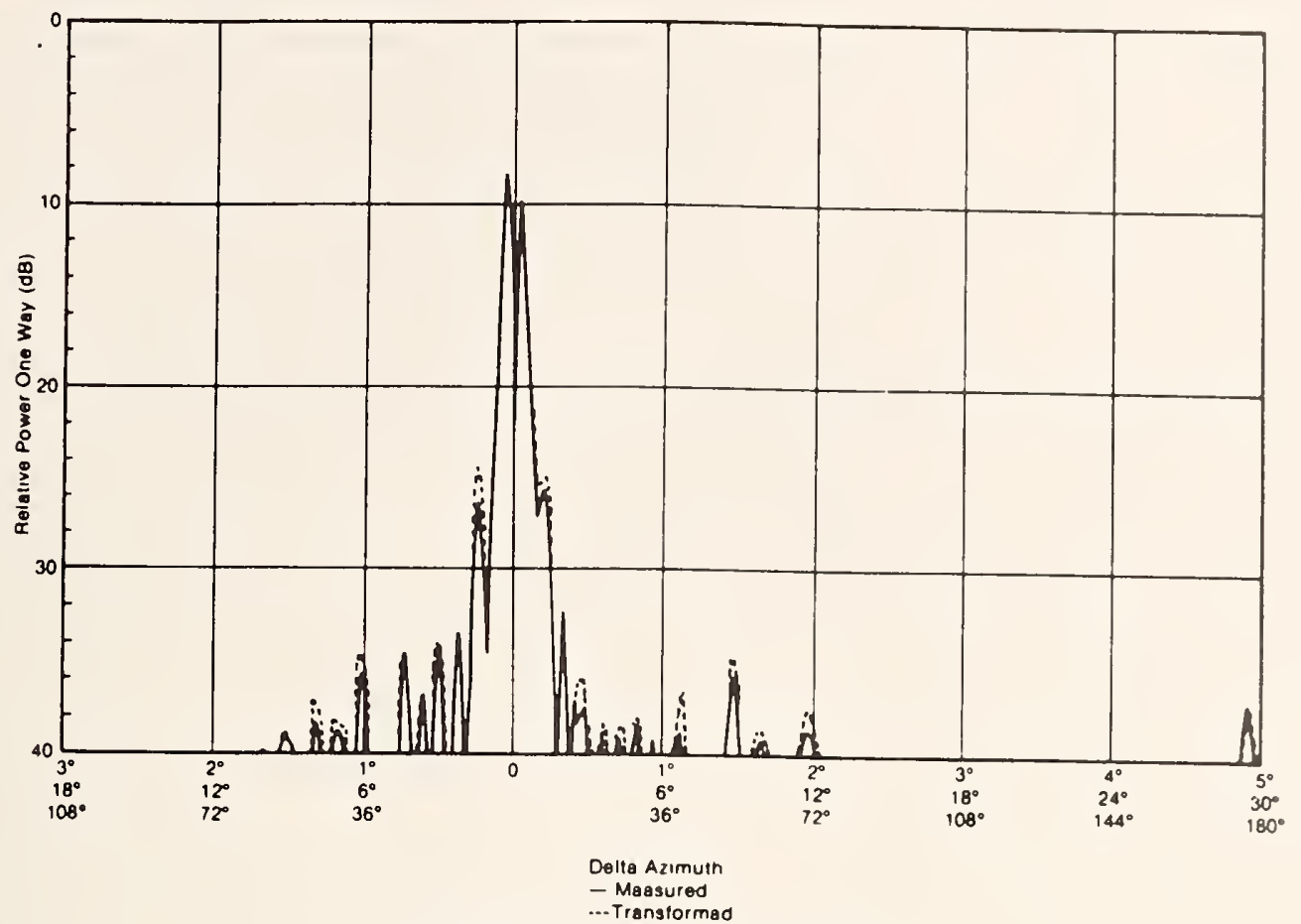

Figure 9-7. Delta azimuth.

figure reproduced from [46] by permission

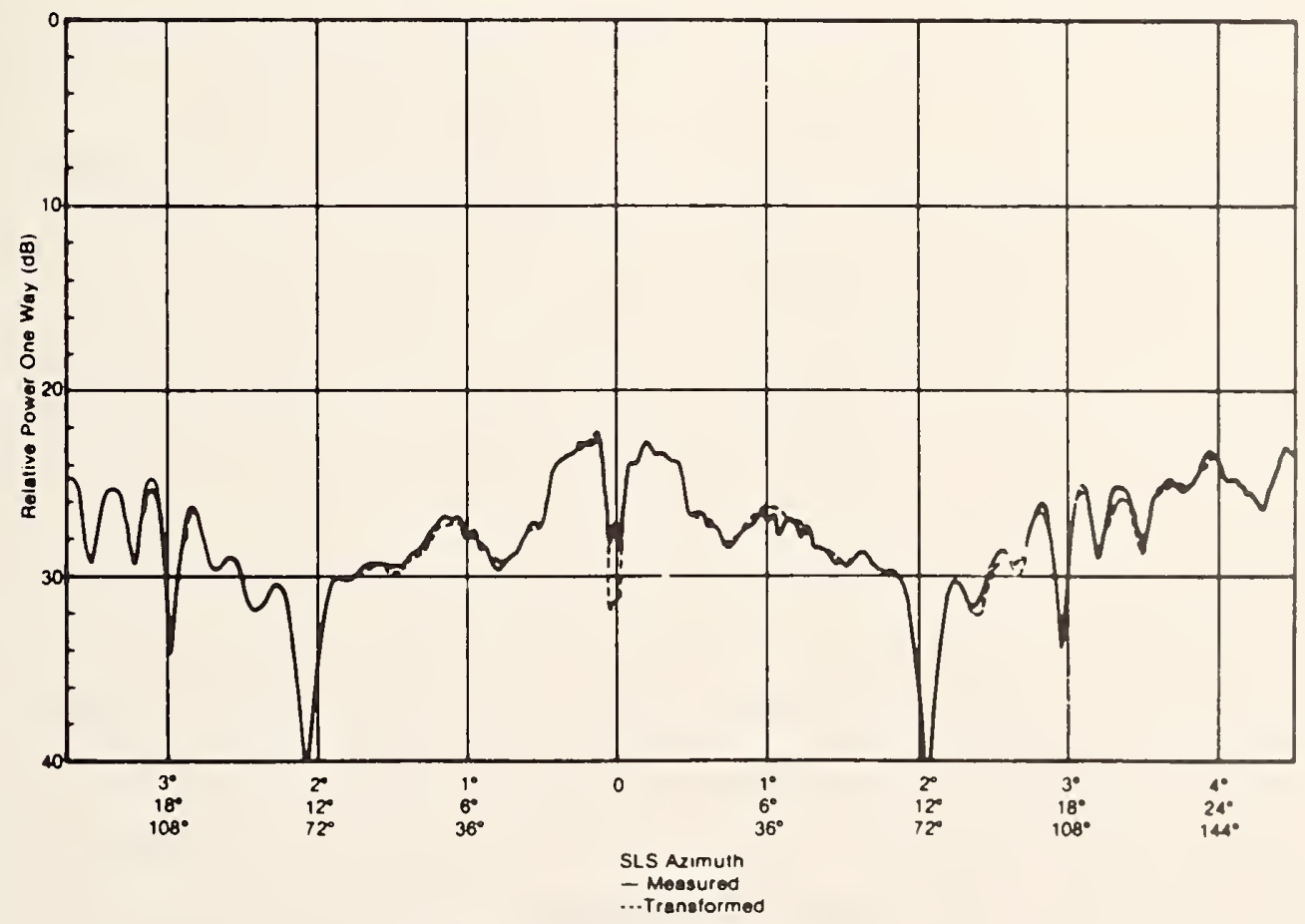

Figure 9.8. SLS azimuth 
In this chapter, a number of numerical comparison tests are presented for validating our implementation of the spherical-scanning algorithm presented in Chapter VIII. In passing these tests, all aspects of the computation algorithm were subjected to rigorous self-consistency verification. A specific self-consistency test showed that the algorithm would function as an identity transformation when $\overline{\hat{R}}_{s \mu n}^{\prime}=\hat{R}_{s \mu n}^{\prime}$. Another test verified the algorithm's computational accuracy in computing known mathematical functions. A natural self-consistency check is to verify that known modal-expansion coefficients result from using analytical formulas to generate input data. One such test simulated displaced-dipole antenna patterns and then numerically generated modal-expansion coefficients for comparison against known results. We also described a test of the probe's translation algorithm that used actual near-field measurements with analytical probe data to produce two identical far-field patterns, even though they were generated using two different measurement-sphere radii and two different sets of probe coefficients corresponding to an original and a translated probe.

In conjunction with these self-consistency tests, a number of interesting analytical results were developed in this chapter. Equation (9.1) evaluates the matrix coupling eq (8.92), which rclates orthogonality-integration coefficients $\dot{W}_{n}^{m \mu}$ to far-field modal summation coefficients $S_{n}^{m \mu}$, for the special case of an ideal-dipole near-field probe whose dipole moment parallels the algorithm's simulated far-field dipole. Applying eq (9.1) effectively converts the sphericalscanning algorithm to the "no probe correction case," thereby creating an algorithm which can be useful for computing a probe's spherical transmitting coefficients from measured data. Also, eqs (9.20) and (9.22) show that decoupling of the modal-expansion coefficients $Q^{\text {smn }}$ by the sphericalscanning algorithm is equivalent to applying the orthogonality relation (2.39) between transverse vector spherical-wave functions to the spherical-wave expansion for the radiated electric field. This result completes our proof that the spherical-scanning algorithm encompasses the "no probe correction" casc, and it also shows that if to first order the probe emulates a y-directed dipole, then the data's measured $\theta$ component should be identified with a probc-rotation angle $\chi$ of $90^{\circ}$, while the data's measured $\phi$ component should bc identified with a probe-rotation angle $\chi$ of $180^{\circ}$. This chapter further showed that with measured probe data hypothetical circumscribing sphercs about the probe and the test antenna must fit within the spherical measurement region without overlapping in order for the probe-translation algorithm to converge. This resulted in eq (9.14) which establishes a truncation point for the probe-translation summation in eq (5.3).

In addition to self-consistency testing, the computer algorithm was used in an independent comparison of computed and measured far-ficld patterns that exhibited excellent correlation with each other [46]. Some graphical rcsults showing the details of this comparison are presented. 


\section{CHAPTER X}

\section{CONCLUSIONS}

This work presents a spherical-wave souree-scattering matrix theory of antennas and antenna-antenna interactions. In this context, an antenna's source-scattering matrix characterizcs the transmitting, receiving, and scattering properties of the antenna. Coordinate-systcm transformation of these source-seattering matrix elements enables us to use these quantitics to describe antenna-antenna interactions, from which efficient near-field spherical-scanning and hemispherical-scanning algorithms are developed.

Chapter I was an introductory chapter which reviewed the eontents of this study.

In Chapter II we introduced notation for representing the field both in the antenna's waveguide feed and in a source-free region beyond a hypothetical sphere enclosing the antenna. The field ineident on a spherieal boundary from an external source was characterized by a dyadic Green's function representation, with particular attention paid to excitation by an external dipole source or a propagating plane wave. We also introduced functional relationships between positive and negative $\mathrm{m}$-index vector spherical-wave functions, along with asymptotic representations and orthogonality relations for these functions.

Chapter III introduced the spherical-wave souree-seattering matrix represcntation of an antenna. Reciproeity relations between the source-scattering matrix elements were obtained for reeiprocal antennas. Next, expressions for power gain and directivity of a transmitting antcnna and receiving eross seetion of a receiving antenna were obtained in terms of the antenna's souree-seattering matrix elements. With a reeiproeal antenna, these results were shown to reduce to the classical relationship between receiving cross seetion and power gain.

Chapter IV presented the basic equations for a rotation of coordinates transformation. Each representation coefficient of this transformation was expressed as a linite Fourier series whosc elements can be calculated by a numerically-stable threc-term recursion rclation. Next, expressions were developed relating a representation coefficient from onc column of the representation-eofficient matrix to the associated Legendre funetion and its derivative. Chapter IV also presented basic cquations for a translation of coordinates transformation. wherein the vector spherical-wave function translation coefficients of lowest order were shown to be simply 
expressed as a spherical Hankel function or its derivative. This chapter concluded with an efficient algorithm for calculating relevant translation coefficients.

Chapter $\mathrm{V}$ developed a formulation for expressing the mutual coupling between two antennas when each antenna is described by a source-scattering matrix related to that antenna's preferred coordinate system. The functional transformations introduced in the previous chapter to describe the effects of coordinate-system rotation and translation were used to transform source-scattering matrix elements between these two preferred coordinate systems. Transformed source-scattering matrix representations for each antenna were then given with respect to the coordinate system of the other antenna. This chapter concluded with the development of a Liouville-Neumann series representation for the elements of the coupled antenna-system's twoport scattering matrix.

Chapter VI established equalities between contragradient vector-wave function translation coefficients, leading to the development of a simple reciprocity relation for reciprocal antennas when the propagation directions are reversed and multiple reflections are negligible. From this, it follows that if the probe is reciprocal then a single algorithm suffices for obtaining the transmitting or receiving pattern of a nonreciprocal antenna from near-field measurements. Next, a relationship was established between transmitting and receiving scattering-matrix elements for a reciprocal probe which includes the effect of a $180^{\circ}$ flip of the probe's alignment along the z-axis. This enables us to obtain probe coefficients for spherical scanning when the transmitting coefficients of the probe with respect to test-antenna coordinates are known. Chapter VI concluded with a proof that interchanging the orders of summation of infinite double serics in Chapter $\mathrm{V}$ is valid, and in so doing established a coordinate-system translation theorem for the dyadic Green's function.

Chapter VII established that our two-antenna coupling equations when one antenna is an ideal dipole arc mutually consistent with the expressions obtained in Chapter II for the fields at the surface of a sphere, whether due to radiation from an enclosed antenna or the incident field of an external dipole source. This chapter starts by developing modal-expansion coefficients for an ideal-dipole source. Specializing to a dipole at the coordinate-system origin and translating coordinates, we obtain translated-dipole receiving coefficients. Inserting these results into our two-antcnna coupling equation, we rcobtain our Chapter II expressions for the transverse componcnts of the radiated electric field. Next, we show that when the receiving antenna is an ideal dipole then our two-antenna coupling equation can be expressed as a dot product between the recciving-dipole moment and the radiated electric field. Then, rcversing the propagation 
direction we show that the signal received at the test antenna can be expressed as a dot product between the transmitting-dipole moment and a modal-excitation vector, consistent with our Chapter II result expressing the modal-excitation coefficients at a spherical surface due to an external dipole source. Applying the reciprocity relation to this expression for the signal received by the test antenna, we reobtain our earlier expression for the signal received by a dipole. Consequently, this chapter demonstrates that when one antenna is an ideal dipole, then the antenna-coupling equations in Chapter $\mathrm{V}$ are compatible with the electric-field expressions given in Chapter II.

In Chapter VIII, algorithms are developed for decoupling the modal-expansion coefficients from the two-antenna coupling equation for a particular propagation direction. Our analysis starts by assuming continuous coupling data is known over the surface of a hypothetical sphere enclosing the test antenna, and an algorithm is developed for this case. A realistic algorithm for measured data is developed from this by assuming discrete data and a circularly-symmetric probe. Improvements to this basic algorithm are then introduced, resulting in an efficient algorithm for calculating far-field antenna patterns from measured near-field data. Finally, an algorithm is developed for halving the computational effort when the test-antenna's field is negligible on onehalf of the measurement sphere, thus producing a hemispherical-scanning algorithm.

In Chapter IX we describe a number of self-consistency tests used to validate all aspects of the computer program implementing the spherical-scanning algorithm developed in Chapter VIII. Moreover, we recount particulars from an experimental verification carried out by a user of this computer program. In addition, we present a modified matrix coupling equation for use with the spherical-scanning algorithm which converts the algorithm to the "no probe correction" case, which may then be used to compute a probe's spherical transmitting coefficients from measured data. We also show that convergence of the series summation for calculating translated-probe coefficients using measured probe data requires the nonoverlapping of circumscribing spheres about the probe and the test antenna within the measurement-sphere region. We go on to show that with an ideal-dipole probe our Chapter VIII expressions for obtaining the test-antenna's modal-expansion coefficients are equivalent to the result that would be obtained by applying orthogonality relations between the vector spherical-wave functions to the Chapter II expression for the radiated electric field. Also, when the probe emulates a $y$-directed dipole it was shown that the $\theta$ and $\phi$ components of the measured data should respectively be identified with probe-rotation angles $\chi$ of $90^{\circ}$ and $180^{\circ}$. 


\section{BIBLIOGRAPHY}

[1] Abramowitz, M.; Stegan, I.A. Handbook of Mathematical Functions. Nat. Bur. Stand. (U.S.) Appl. Math. Ser. 55; June 1964.

[2] Ashton, R.W.; et al. A study of the prediction of antenna performances from near field measurements. Marconi Tech. Rept. MTR 75/43; June 1975.

[3] Batemann Manuscript Project Staff, A. Erdélyi, ed. Higher Transcendental Functions I. McGraw Hill; 1953.

[4] Batemann Manuscript Project Staff, A. Erdélyi, ed. Higher Transcendental Functions II. McGraw Hill; 1953.

[5] Booker, H.G.; Clemmow, P.C. The concept of an angular spectrum of plane waves, and its relation to that of polar diagram and aperture distribution. Proc. IEE 97, part III: 11-17; January 1950.

[6] Bromwich, T.J.I., An Introduction to the Theory of Infinite Series. MacMillan \& Co. Second Edition, Revised 1955.

[7] Brown, J. Theoretical analysis of some errors in aerial measurements. Proc. IEE 105C: 343351; February 1958.

[8] Brown, J.; Jull, E.V. The prediction of aerial radiation patterns from near-field measurements. Proc. IEE 108B: 635-644; November 1961.

[9] Bruning, J.H.; Lo, Y.T. Multiple scattering by spheres. Antenna Lab., University of Illinois, Urbana, Illinois, Tech. Rept. 69-5; May 1969.

[10] Bruning, J.H.; Lo, Y.T. Multiple scattering of EM waves by spheres, Part I: multipole expansions and ray-optical solutions, IEEE Trans. Antennas Propagat. AP-19(3): 378-390; 1971.

[11] Cochran, W.T., et al. What is the fast Fourier transform? Proc. IEEE 55(10): 1664-1674; October 1967.

[12] Collin, R.E.; Zucker, F.J. Antenna Theory, I. McGraw Hill; 1969.

[13] Cooley, J.W.; Lewis, P.A.W.; Welch, P.D. The fast Fourier transform algorithm and its applications. IBM Research Paper RC-1743; February 9, 1967.

[14] Cooley, J.W.; Tukey, J.W.; An algorithm for the machine calculation of complex Fourier series. Math. of Computation 19: 297-301; April 1965. 
[15] Cruzan, O.R. Translational addition theorems for spherical vector wave functions. Quart. Appl. Math. 20(1): 33-40; 1962; and Cruzan, O.R. Translational addition theorems for spherical vector wave functions. Diamond Ordnance Fuze Laboratories, Department of the Army, TR-906; March 10, 1961.

[16] Danos, M.; Maximon, L.C. Multipole matrix elements of the translation operator. J. Math. Phys. 6(1): 766-778; 1965.

[17] Edmonds, A.R. Angular Momentum in Quantum Mechanics. Princeton University Press; third printing, with corrections; 1974.

[18] Fano, U.; Racah, G. Irreducible Tensorial Sets. Academic Press; 1959.

[19] Friedman, B.; Russek, J. Addition theorems for spherical waves. Quart. Appl. Math. 12(1): $13-23 ; 1954$.

[20] Gautschi, W. Computational aspects of three term recurrence relations. SIAM Review 9(1): 24-82; 1967.

[21] Jensen, F. Electromagnetic near-field far-field correlations. Ph.D. dissertation, Technical University of Denmark, Lyngby, Denmark; July 1970.

[22] Johnson, R.C.; Ecker, A.H.; Hollis, S.J. Determination of far-field antenna patterns from near-field measurements. Proc. IEEE 61(12): 1668-1698; 1973.

[23] Jones, D.S. The Theory of Electromagnetism. Pergamon Press; 1964.

[24] Jones, D.S. Methods in Electromagnetic Wave Propagation. Clarendon Press; 1979.

[25] Kerns, D.M. Plane-wave scattering matrix theory of antennas and antenna-antenna interactions. J. Res. Nat. Bur. Stand. (U.S.) 80B(1): 5-51; 1976.

[26] Kerns, D.M. Plane-wave scattering matrix theory of antennas and antenna-antenna interactions. Nat. Bur. Stand. (U.S.) Monogr. 162; June 1981.

[27] Kerns, D.M.; Beatty, R.W. Basic Theory of Waveguide Junctions and Introductory Microwave Network Analysis. Pergamon Press; 1967.

[28] Kerns, D.M.; Dayhoff, E.S. Theory of diffraction in microwave interferometry. J. Res. Nat. Bur. Stand. (U.S.) 64B(1): 1-13; 1960.

[29] Kahn, W.V.; Kurss, H. Minimum scattering antennas. IEEE Trans. Antennas Propagat. AP13: 671-675; September 1965.

[30] Kummer, W.H.; Gillespie, E.S. Antenna measurements-1978. Proc. IEEE 66(4): 483-507: April 1978.

[31] Larsen, F.H. Improved algorithm for probe corrected spherical near-field/far-field transformation. Electron. Lett. 15(19): 588-590; September 1979. 
[32] Larsen, F.H. Probe correction of spherical near-field measurements of a satellite model. Proc., Workshop on Antenna Testing Techniques, ESTEC, Holland, pp. 123-128, 6-8 June 1977.

[33] Leach, W.M., Jr.; Paris, D.T. Probe compensated near-field measurements on a cylinder. IEEE Trans. Antennas Propagat. AP-21(4): 435-445; 1973.

[34] Lewis, R.L. Recursion formula for the logarithmic derivatives of spherical Bessel functions in the complex plane. U.S. Dept. of Commerce ESSA Tech. Rept. 151-ITS-44; January 1970.

[35] Lewis, R.L. Highly efficient processing for near-field spherical scanning data reduction. AP-S Intl. Symp., University of Massachusetts, Amherst, Massachusetts, IEEE Cat. 76 CH1121-3AP, 11-15 October 1976.

[36]-A Lewis, R.L. Spherical-wave source-scattering matrix analysis of coupled antennas: a general system two-port solution. IEEE Trans. Antennas Propagat. AP-35(12); 1375-1380; 1987.

[36]-B Lewis, R.L.; Wittmann, R. C. Improved spherical and hemispherical scanning algorithms. IEEE Trans. Antennas Propagat. AP-35(12); 1381-1388; 1987.

[37] Lewis, R.L. The use of three term recursion relations for numerical computations as applied to near-field spherical scanning. Proc. URSI Symp. on Electromagnetic Wave Theory, Stanford University, Stanford, California; June 20-24, 1977.

[38] Liang, C.; Lo, Y.T. Scattering by two spheres. Radio Science 2 (new series) (12): 1481-1495; December 1967.

[39] Ludwig, A.C. Near-field far-field transformations using spherical-wave expansions. IEEE Trans. Antennas Propagat. AP-19: 214-220; 1971.

[40] Montgomery, C.G.; Dicke, R.H.; Purcell, E.M. Principles of Microwave Circuits. Radiation Laboratory of MIT Series, Vol. 8, McGraw Hill; 1948.

[41] Rahmat-Samii, Y.; Mittra, R.; Israel, V.G. Computation of Fresnel and Fraunhofer fields of planar apertures and reflector antennas by the Jacobi-Bessel Series-a review. Electromagn. 1(2): 155-185; April-June 1981.

[42] Ramo, S.; Whinnery, J.R.; Van Duzer, T. Fields and Waves in Communication Electronics. Wiley \& Sons; 1965.

[43] Rose, M.E. Multipole Fields. Wiley \& Sons; 1955.

[44] Rose, M.E. Elementary Theory of Angular Momentum. Wiley \& Sons; 1957.

[45] Row, R.V. Theoretical and experimental study of electromagnetic scattering by two identical conducting cylinders. J. Appl. Phys. 26(6): 666-675; 1955. 
[46] Sanzgiri, S.; Lee, K. Spherical and cylindrical near-field testing of electrically large antennas. 1981 Antenna Applications Symposium, Allerton Park, Monticello, Illinois, September 23-25, 1981.

[47] Silverman, H.F. Fast Fourier transform methods. IBM Research Rept. RC3747; February 1972.

[48] Stakgold, I. Boundary Value Problems of Mathematical Physics, I. MacMillan \& Co.; 1967.

[49] Stein, S. Addition theorems for spherical wave functions. Quart. Appl. Math. 19(1): 15-24; 1961; and, Stein, S. Addition theorems for spherical wave functions. Hermes Electrical Co., Cambridge, Massachusetts. Tech. Rept. No. 1 under Nonr 2632(00) to Office Naval Research, Hermes Electrical Co. Rept. M697; 15 August 1959.

[50] Stratton, J.A. Electromagnetic Theory. McGraw-Hill; 1941.

[51] Tai, Chen-To. Dyadic Green's Functions in Electromagnetic Theory. Intl. Textbook Co.; 1971.

[52] Tai, Chen-To. Eigen-function expansion of dyadic Green's Functions. Mathematics Note Series; Note 28; July 1973.

[53] Twersky, V. Multiple scattering of radiation, Part I. New York University Research Rept. EM-34; July 1951.

[54] Wacker, P.F. Non-planar near-field measurements. Internal NBS reports to Air Force Avionics Lab., Wright-Patterson Air Force Base, Dayton, Ohio. First Progress Rept.; 31 January 1974. Second Progress Rept.; 28 May 1974.

[55] Wacker, P.F. Near-field antenna measurements using a spherical scan: Efficient data reduction with probe correction. Conf. on Precision Electromagnetic Measurements, IEE Conf. Publ. 113, IEEE Cat. 74CH0 770-8IM; IEE 1-5 July 1974.

[56] Wacker, P.F. Non-planar near-field measurements: Spherical scanning. Nat. Bur. Stand. (U.S.) NBSIR 75-809; June 1975.

[57] Wasylkiwskyj, W; Kahn, W.A. Theory of mutual coupling among minimum scattering antennas. IEEE Trans. Antennas Propagat. AP-18(2): 204-216; March 1970.

[58] Wigner, E.P. (translated by Griffin, J.J.) Group Theory. Academic Press; 1959.

[59] Wittmann, R.C.; Stubenrauch, C.F. Spherical near-field scanning: experimental and theoretical studies. Natl. Inst. Stand. Technol. NISTIR 3955; July 1990.

[60] Yaghjian, A.D. Near-field antenna measurements on a cylindrical surface: a source scattering-matrix formulation. Nat. Bur. Stand. (U.S.) Tech. Note 696: September 1977. 


\section{APPENDIX A}

\section{EXPANSION OF AN INCIDENT PLANE-WAVE FIELD AS A SERIES OF VECTOR SPHERICAL-WAVE FUNCTIONS}

The purpose of this appendix is to present a vector spherical-wave-function seriesexpansion representation for an incident plane-wave field, where the expansion coefficients are obtained from the orthogonality relations between the vector spherical-wave functions by analytically evaluating integrals of the scalar dot product of these vector spherical-wave functions with the incident-field vector. As a result, the derivation to be presented here avoids any consideration of a dyadic Green's function representation of the incident fields. Our problem assumes an arbitrary orientation of the incident plane-wave field with respect to the sphericalcoordinate system in which the vector spherical-wave functions are defined. The analysis to be presented is partly based upon a related formulation [23, pp. 487-494] which stopped after the evaluation of one of the two required integrals and which treated the case of a radiating dipole source rather than an incident plane-wave field. Furthermore, the results obtained in ref. [23] cannot be used directly here because of differences in conventions, including the use of absolutevalue bars on the associated Legendre function indices. Consequently, our current derivation is necessary for the validation of equivalent results obtained in section 2.3. Because the problem we are attacking involves a great deal of algebraic manipulation, we shall just present highlights of the derivation rather than lay out every individual step.

We start by specifying the incident electromagnetic field as

$$
\underline{E}=-\mathscr{E}_{0} \underline{\hat{e}} e^{-i \underline{k} \cdot \underline{r}}, \underline{H}=\eta \mathscr{E}_{0} \underline{\hat{h}} e^{-i \underline{k} \cdot \underline{r}},
$$

where $\mathscr{E}_{0}$ is an arbitrary constant; $\eta=\sqrt{\frac{\epsilon}{\mu}}$ is the free-space wave admittance while $k=\omega \sqrt{\mu \epsilon}$ designates the free-space propagation constant; $\underline{r}$ designates the position coordinate vector from the coordinate-system origin to a point in space; $\underline{\hat{e}}, \underline{\hat{h}}, \underline{\hat{k}}$ represent a right-hand triad of unit vectors such that $\underline{\hat{e}} \times \underline{\hat{h}}=\underline{\hat{k}}$, and $\underline{k}=k \underline{\hat{k}}$. For the balance of the present discussion, we shall set $\mathscr{E}_{0}=1$.

We next need to consider how the unit vectors $\underline{\hat{e}}, \underline{\hat{h}}, \underline{\hat{k}}$ are related to the unit vectors of the spherical-coordinate system. To this end, if $\underline{a}_{x}, \underline{a}_{y}, \underline{a}_{z}$ denote the usual cartesian-coordinate unit vectors we have the relationship 


$$
\left[\begin{array}{l}
\underline{a}_{x} \\
\underline{a}_{y} \\
\underline{a}_{z}
\end{array}\right]=\left[\begin{array}{ccc}
\sin \theta \cos \phi & \cos \theta \cos \Phi & -\sin \phi \\
\sin \theta \sin \phi & \cos \theta \sin \Phi & \cos \phi \\
\cos \theta & -\sin \theta & 0
\end{array}\right]\left[\begin{array}{l}
\underline{a}_{r} \\
\underline{a}_{\theta} \\
\underline{a}_{\phi}
\end{array}\right]
$$

We now express the relation between the unit vectors $\hat{\hat{e}}, \underline{\hat{h}}, \hat{k}$ and the cartesian-coordinate unit vectors as

$$
\left[\begin{array}{c}
\hat{\hat{e}} \\
\underline{\hat{h}} \\
\hat{\hat{k}}
\end{array}\right]=\left[\begin{array}{ccc}
\cos \gamma & \sin \gamma & 0 \\
-\sin \gamma & \cos \gamma & 0 \\
0 & 0 & 1
\end{array}\right]\left[\begin{array}{ccc}
\cos \beta & 0 & -\sin \beta \\
0 & 1 & 0 \\
\sin \beta & 0 & \cos \beta
\end{array}\right]\left[\begin{array}{ccc}
\cos \alpha & \sin \alpha & 0 \\
-\sin \alpha & \cos \alpha & 0 \\
0 & 0 & 1
\end{array}\right]\left[\begin{array}{l}
\underline{a}_{x} \\
\underline{a}_{y} \\
\underline{a}_{z}
\end{array}\right] .
$$

It may be noted that eq (A-3) agrees with the rotation of coordinates transformation matrix developed by Rose [44]. Now upon combining eqs (A.2) and (A.3), we obtain

$$
\begin{aligned}
\underline{\hat{e}}= & -[\cos \gamma(\sin \beta \cos \theta-\cos \beta \sin \theta \cos \psi)-\sin \gamma \sin \theta \sin \psi] \underline{a}_{r} \\
& +[\cos \gamma(\sin \beta \sin \theta+\cos \beta \cos \theta \cos \psi)+\sin \gamma \cos \theta \sin \psi] \underline{a}_{\theta} \\
& -[\cos \gamma \cos \beta \sin \psi-\sin \gamma \cos \psi] \underline{a}_{\phi},
\end{aligned}
$$

and

$$
\underline{\hat{k}} \cdot \underline{a}_{r}=\cos \beta \cos \theta+\sin \beta \sin \theta \cos \psi,
$$

where we have introduced the notation $\psi=\phi-\alpha$. The components of $\underline{\hat{h}}$ and the other components of $\underline{\hat{k}}$ are not needed, although they could readily be obtained.

Now, from ref. [50, eq (57), p. 409], and using eq (A.5) we have

$$
e^{-i k \cdot L}=\sum_{n=0}^{\infty}(2 n+1)(-i)^{n} j_{n}(k r) \sum_{m=-n}^{n} \frac{(n-m) !}{(n+m) !} P_{n}^{m}(\cos \beta) P_{n}^{m}(\cos \theta) e^{-i m \psi} .
$$

Consequently, from the orthogonality properties of the associated Legendre functions [3], we obtain [19]

$$
\int_{0}^{2 \pi} \int_{0}^{\pi} e^{-i \underline{k} \cdot r} P_{n}^{m}(\cos \theta) e^{i m \psi} \sin \theta d \theta d \phi=4 \pi i^{-n} j_{n}(k r) P_{n}^{m}(\cos \beta) .
$$

Upon using the definitions (2.7) for the vector spherical-wave functions, we can write the incident electric field in eq (A.1) as the series expansion

$$
\underline{E}=\sum_{n=1}^{\infty} \sum_{m=-n}^{n}\left[p_{m n} \underline{N}_{m n}+q_{m n} \underline{M}_{m n}\right],
$$

where $\mathrm{p}_{\mathrm{m} n}$ and $\mathrm{q}_{\mathrm{m}}$ are to be determined. Now, upon reversing the order of summation on the m-index in eq (A.7) and using eq (2.24) along with the orthogonality relations (2.38), we obtain 


$$
\begin{aligned}
X_{m n} & \equiv e^{-i m \alpha} \sqrt{\frac{(n+m) !}{(n-m) !}} \int_{0}^{2 \pi} \int_{0}^{\pi} \underline{N}_{m n} \cdot \underline{E} \sin \theta d \theta d \phi \\
& =(-)^{m} 4 \pi \frac{n(n+1)}{(2 n+1)^{2}}\left\{(n+1)\left[j_{n-1}(k r)\right]^{2}+n\left[j_{n+1}(k r)\right]^{2}\right\} e^{-i m \alpha} \sqrt{\frac{(n+m) !}{(n-m) !}} p_{-m, n}
\end{aligned}
$$

and

$$
\begin{aligned}
Y_{m n} & \equiv e^{-i m \alpha} \sqrt{\frac{(n+m) !}{(n-m) !}} \int_{0}^{2 \pi} \int_{0}^{\pi} \underline{M}_{m n} \cdot \underline{E} \sin \theta d \theta d \phi \\
& =(-)^{m} 4 \pi \frac{n(n+1)}{2 n+1}\left[j_{n}(k r)\right]^{2} e^{-i m \alpha} \sqrt{\frac{(n+m) !}{(n-m) !}} q_{-m, n} .
\end{aligned}
$$

Thus, it remains to evaluate $X_{m n}$ and $Y_{m n}$ for $\underline{E}$ given by eq (A.1). Starting with the evaluation of $Y_{n n}$, from eq (A.4) we obtain

$$
\begin{gathered}
Y_{m n}=-j_{n}(k r)\left\{\operatorname { c o s } \gamma \int _ { 0 } ^ { 2 \pi } d \phi \int _ { 0 } ^ { \pi } d \theta \operatorname { s i n } \theta e ^ { - i k \cdot \tau } \left[(\sin \beta \sin \theta+\cos \beta \cos \theta \cos \psi) \frac{i m P_{n}^{m}(\cos \theta)}{\sin \theta}\right.\right. \\
\left.+\cos \beta \sin \psi \frac{\partial P_{n}^{m}(\cos \theta)}{\partial \theta}\right] e^{i m \psi} \\
\left.+\sin \gamma \int_{0}^{2 \pi} d \phi \int_{0}^{\pi} d \theta \sin \theta e^{-i k \cdot \tau}\left[\cos \theta \sin \psi \frac{i m P_{n}^{m}(\cos \theta)}{\sin \theta}-\cos \psi \frac{\partial P_{n}^{m}(\cos \theta)}{\partial \theta}\right] e^{i m \psi}\right\} .
\end{gathered}
$$

Our goal is to evaluate the double integrals in this expression using eq (A.6); however, as the equation stands there is only one term that can be so evaluated. The procedure to be used follows almost exactly a related procedure by Jones [23, p. 493]. After separating out the one term that fits the formula (A.6), we make the replacements $\sin \psi=\frac{1}{2 i}\left(e^{i \psi}-e^{-i \psi}\right)$ and $\cos \psi=\frac{1}{2}\left(e^{i \psi}+e^{-i \psi}\right)$; then like coefficients of $e^{i \psi}$ and $e^{-i \psi}$ are collected, whereupon we can use the relations

$$
\begin{aligned}
& \sin \theta \frac{\partial P_{n}^{m}(\cos \theta)}{\partial \theta}-m \cos \theta P_{n}^{m}(\cos \theta)=\sin \theta P_{n}^{m+1}(\cos \theta) \\
& \sin \theta \frac{\partial P_{n}^{m}(\cos \theta)}{\partial \theta}+m \cos \theta P_{n}^{m}(\cos \theta)=-(n+m)(n-m+1) \sin \theta P_{n}^{m-1}(\cos \theta) .
\end{aligned}
$$

The two expressions (A.10a) and (A.10b) follow from taking linear combinations of eqs (4.20a) and $(4.20 \mathrm{~b})$. Upon carrying through the indicated process, the following expression is obtained 


$$
\begin{aligned}
& Y_{m n}=\left[j_{n}(k r)\right]^{2} 4 \pi m i^{-n-1} \cos \gamma \sin \beta P_{n}^{m}(\cos \beta) \\
& +\frac{i}{2} j_{n}(k r) \cos \gamma \cos \beta \int_{0}^{2 \pi} d \phi \int_{0}^{\pi} d \theta \sin \theta e^{-i k \cdot \tau}\left[e^{i \psi} P_{n}^{m+1}(\cos \theta)+(n+m)(n-m+1) e^{-i \psi} P_{n}^{m-1}(\cos \theta)\right] e^{i m \psi} \\
& +\frac{1}{2} j_{n}(k r) \sin \gamma \int_{0}^{2 \pi} d \phi \int_{0}^{\pi} d \theta \sin \theta e^{-i k \cdot \tau}\left[e^{i \psi} P_{n}^{m+1}(\cos \theta)-(n+m)(n-m+1) e^{-i \psi} P_{n}^{m-1}(\cos \theta)\right] e^{i m \psi}
\end{aligned}
$$

Since all of integrals in the preceding expression are of the form (A.6), the integration can readily be carried out. Then, upon replacing $\theta$ by $\beta$ in eq (A.10) and substituting the resulting formulas into our new expression for $\mathrm{Y}_{\mathrm{mn}}$, the final result is obtained in terms of associated Legendre functions of index $m$ rather than in terms of the indices $m \pm 1$. Thus, we obtain

$$
Y_{m n}=-4 \pi i^{-n}\left[j_{n}(k r)\right]^{2}\left\{\frac{i m P_{n}^{m}(\cos \beta)}{\sin \beta} \cos \gamma-\frac{\partial P_{n}^{m}(\cos \beta)}{\partial \beta} \sin \gamma\right\} \text {. }
$$

Consequently, upon equating eqs (A.12) and (A.9), we obtain

$$
q_{-m, n}=(-)^{m+1} i^{-n} \frac{2 n+1}{n(n+1)} \underline{G}_{n}^{1 m}(\beta, \alpha) \cdot\left(\cos \gamma \underline{a}_{\beta}+\sin \gamma \underline{a}_{\alpha}\right) \text {, }
$$

where we have used the definition (2.31). Then, using eq (2.30), we obtain the following expression for the expansion coefficient:

$$
q_{m n}=-i^{-n} \frac{2 n+1}{n(n+1)} \underline{G}_{n}^{1 m *}(\beta, \alpha) \cdot\left(\cos \gamma \underline{a}_{\beta}+\sin \gamma \underline{a}_{\alpha}\right) \text {. }
$$

This completes our evaluation for the case of the expansion coefficients of the $\underline{M}_{m n}$ vector spherical-wave functions; it may be noted that a similar evaluation was carried out by Jones [23].

We now turn our attention to the integral $\mathrm{X}_{\mathrm{mn}}$, for which there does not appear to be any previously published detailed evaluation. Upon substituting the explicit components of $\underline{N}_{m a}$ into eq (A.8) and using eq (A.4), we obtain

$$
\begin{gathered}
X_{m n}=\frac{n(n+1)}{k r} j_{n}(k r) \int_{0}^{2 \pi} d \phi \int_{0}^{\pi} d \theta \sin \theta e^{-i k \cdot \tau}[\cos \gamma(\sin \beta \cos \theta-\cos \beta \sin \theta \cos \psi) \\
-\sin \gamma \sin \theta \sin \phi] P_{n}^{m}(\cos \theta) e^{i m \psi} \\
-\frac{1}{k r} \frac{\partial}{\partial r}\left[r j_{n}(k r)\right]\left\{\operatorname { c o s } \gamma \int _ { 0 } ^ { 2 \pi } d \phi \int _ { 0 } ^ { \pi } d \theta \operatorname { s i n } \theta e ^ { - i k \cdot \tau } \left[(\sin \beta \sin \theta+\cos \beta \cos \theta \cos \psi) \frac{\partial P_{n}^{m}(\cos \theta)}{\partial \theta}\right.\right. \\
\left.\quad-\cos \beta \sin \psi \frac{i m P_{n}^{m}(\cos \theta)}{\sin \theta}\right] e^{i m \psi} \\
\left.+\sin \gamma \int_{0}^{2 \pi} d \phi \int_{0}^{\pi} d \theta \sin \theta e^{-i \xi \cdot \tau}\left[\cos \theta \sin \psi \frac{\partial P_{n}^{m}(\cos \theta)}{\partial \theta}+\cos \psi \frac{i m P_{n}^{m}(\cos \theta)}{\sin \theta}\right] e^{i m \psi}\right\} .
\end{gathered}
$$


We next use the relations between contiguous spherical Bessel functions [1] to obtain

$$
\begin{aligned}
& X_{m n}=\frac{\cos \gamma \sin \beta}{2 n+1}\left\{(n+1) j_{n-1}(k r) \int_{0}^{2 \pi} d \phi \int_{0}^{\pi} d \theta \sin \theta e^{-i \xi \cdot L}\left[n \cos \theta P_{n}^{m}(\cos \theta)-\sin \theta \frac{\partial P_{n}^{m}(\cos \theta)}{\partial \theta}\right] e^{i m \psi}\right. \\
& \left.+n j_{n+1}(k r) \int_{0}^{2 \pi} d \phi \int_{0}^{\pi} d \theta \sin \theta e^{-i \xi \cdot \varepsilon}\left[(n+1) \cos \theta P_{n}^{m}(\cos \theta)+\sin \theta \frac{\partial P_{n}^{m}(\cos \theta)}{\partial \theta}\right] e^{i m \psi}\right\} \\
& -\frac{\cos \gamma \cos \beta}{2 n+1}\left\{( n + 1 ) j _ { n - 1 } ( k r ) \int _ { 0 } ^ { 2 \pi } d \phi \int _ { 0 } ^ { \pi } d \theta \operatorname { s i n } \theta e ^ { - i k \cdot c } \left[\left(n \sin \theta P_{n}^{m}(\cos \theta)+\cos \theta \frac{\partial P_{n}^{m}(\cos \theta)}{\partial \theta}\right) \cos \psi\right.\right. \\
& \left.-\frac{i m P_{n}^{m}(\cos \theta)}{\sin \theta} \sin \psi\right] e^{i m \psi} \\
& +n j_{n+1}(k r) \int_{0}^{2 \pi} d \phi \int_{0}^{\pi} d \theta \sin \theta e^{-i k \cdot t}\left[\left([n+1] \sin \theta P_{n}^{m}(\cos \theta)-\cos \theta \frac{\partial P_{n}^{m}(\cos \theta)}{\partial \theta}\right) \cos \psi\right. \\
& \left.\left.+\frac{i m P_{n}^{m}(\cos \theta)}{\sin \theta} \sin \psi\right] e^{i m \psi}\right\} \\
& -\frac{\sin \gamma}{2 n+1}\left\{( n + 1 ) j _ { n - 1 } ( k r ) \int _ { 0 } ^ { 2 \pi } d \phi \int _ { 0 } ^ { \pi } d \theta \operatorname { s i n } \theta e ^ { - i k \cdot \tau } \left[\left(n \sin \theta P_{n}^{m}(\cos \theta)+\cos \theta \frac{\partial P_{n}^{m}(\cos \theta)}{\partial \theta}\right) \sin \psi\right.\right. \\
& \left.+\frac{i m P_{n}^{m}(\cos \theta)}{\sin \theta} \cos \psi\right] e^{i m \psi} \\
& +n j_{n+1}(k r) \int_{0}^{2 \pi} d \phi \int_{0}^{\pi} d \theta \sin \theta e^{-i \xi \cdot t}\left[\left([n+1] \sin \theta P_{n}^{m}(\cos \theta)-\cos \theta \frac{\partial P_{n}^{m}(\cos \theta)}{\partial \theta}\right) \sin \psi\right. \\
& \left.\left.-\frac{i m P_{n}^{m}(\cos \theta)}{\sin \theta} \cos \psi\right] e^{i m \psi}\right\} \text {. }
\end{aligned}
$$

Now, from the recurrence relations of the associated Legendre functions [3] we obtain

$$
\left[n \sin \theta P_{n}^{m}(\cos \theta)+(\cos \theta) \frac{\partial P_{n}^{m}(\cos \theta)}{\partial \theta}\right] \sin \theta=n P_{n}^{m}(\cos \theta)-(n+m) \cos \theta P_{n-1}^{m}(\cos \theta)
$$

$$
\left[(n+1) \sin \theta P_{n}^{m}(\cos \theta)-\cos \theta \frac{\partial P_{n}^{m}(\cos \theta)}{\partial \theta}\right] \sin \theta=(n+1) P_{n}^{m}(\cos \theta)-(n-m+1) \cos \theta P_{n+1}^{m}(\cos \theta),
$$

and

$$
\begin{gathered}
\left(1-x^{2}\right)^{1 / 2} P_{n-1}^{m+1}(x)=(n-m) P_{n}^{m}(x)-(n+m) x P_{n-1}^{m}(x) \\
(n+m-1)\left(1-x^{2}\right)^{1 / 2} P_{n-1}^{m-1}(x)=x P_{n-1}^{m}(x)-P_{n}^{m}(x) \\
\left(1-x^{2}\right)^{1 / 2} P_{n+1}^{m+1}(x)=(n-m+1) x P_{n+1}^{m}(x)-(n+m+1) P_{n}^{m}(x) \\
(n-m+2)\left(1-x^{2}\right)^{1 / 2} P_{n+1}^{m-1}(x)=P_{n}^{m}(x)-x P_{n+1}^{m}(x) .
\end{gathered}
$$


Combining eqs (A.15) and (A.16), we obtain the expressions

$$
\begin{aligned}
& {\left[n \sin \theta P_{n}^{m}(\cos \theta)+\cos \theta \frac{\partial P_{n}^{m}(\cos \theta)}{\partial \theta}-\frac{m P_{n}^{m}(\cos \theta)}{\sin \theta}\right] e^{i \psi}=P_{n-1}^{m+1}(\cos \theta) e^{i \psi}} \\
& {\left[n \sin \theta P_{n}^{m}(\cos \theta)+\cos \theta \frac{\partial P_{n}^{m}(\cos \theta)}{\partial \theta}+\frac{m P_{n}^{m}(\cos \theta)}{\sin \theta}\right] e^{-i \psi}=-(n+m)(n+m-1) P_{n-1}^{m-1}(\cos \theta) e^{-i \psi}} \\
& {\left[(n+1) \sin \theta P_{n}^{m}(\cos \theta)-\cos \theta \frac{\partial P_{n}^{m}(\cos \theta)}{\partial \theta}+\frac{m P_{n}^{m}(\cos \theta)}{\sin \theta}\right] e^{i \psi}=-P_{n+1}^{m+1}(\cos \theta) e^{i \psi}} \\
& {\left[(n+1) \sin \theta P_{n}^{m}(\cos \theta)-\cos \theta \frac{\partial P_{n}^{m}(\cos \theta)}{\partial \theta}-\frac{m P_{n}^{m}(\cos \theta)}{\sin \theta}\right] e^{-i \psi}=(n-m+1)(n-m+2) P_{n+1}^{m-1}(\cos \theta) e^{-i \psi}}
\end{aligned}
$$

Consequently, upon making the replacements $\sin \psi=1 /(2 \mathrm{i})\left(\mathrm{e}^{\mathrm{i} \psi}-\mathrm{e}^{-\mathrm{i} \psi}\right)$ and $\cos \psi=1 / 2\left(\mathrm{e}^{\mathrm{i} \psi}+\mathrm{e}^{\mathrm{i} i \psi}\right)$ in eq (A.14), we find that the resulting integrands are equal to simple linear combinations of the expressions on the left-hand sides of eq (A.17); hence, all of the integrals in eq (A.14) for $X_{m n}$ can be expressed as integrals of the type given by eq (A.6), so we can proceed just as we did previously in evaluating eq (A.11). Accordingly, we obtain

$$
\begin{aligned}
\frac{2 n+1}{4 \pi} i^{n-1} X_{m n}= & \cos \gamma \sin \beta\left\{(n+1)\left[j_{n-1}(k r)\right]^{2}\left[n \cos \beta P_{n}^{m}(\cos \beta)-\sin \beta \frac{\partial P_{n}^{m}(\cos \beta)}{\partial \beta}\right]\right. \\
& \left.-n\left[j_{n+1}(k r)\right]^{2}\left[(n+1) \cos \beta P_{n}^{m}(\cos \beta)+\sin \beta \frac{\partial P_{n}^{m}(\cos \beta)}{\partial \beta}\right]\right\} \\
-\cos \gamma \cos \beta\left\{(n+1)\left[j_{n-1}(k r)\right]^{2}\left[n \sin \beta P_{n}^{m}(\cos \beta)+\cos \beta \frac{\partial P_{n}^{m}(\cos \beta)}{\partial \beta}\right]\right. & \\
& \left.-n\left[j_{n+1}(k r)\right]^{2}\left[(n+1) \sin \beta P_{n}^{m}(\cos \beta)-\cos \beta \frac{\partial P_{n}^{m}(\cos \beta)}{\partial \beta}\right]\right\} \\
- & i \sin \gamma\left\{(n+1)\left[j_{n-1}(k r)\right]^{2} \frac{m P_{n}^{m}(\cos \beta)}{\sin \beta}+n\left[j_{n+1}(k r)\right]^{2} \frac{m P_{n}^{m}(\cos \beta)}{\partial \beta}\right\} .
\end{aligned}
$$

It is readily seen that most of the terms in the preceding expression either cancel each other or combine upon using the elementary trigonometric formula, $\cos ^{2} \beta+\sin ^{2} \beta=1$. Thus, our final expression becomes 


$$
\begin{gathered}
X_{m n}=\frac{4 \pi}{2 n+1} i^{-n-1}\left\{(n+1)\left[j_{n-1}(k r)\right]^{2}+n\left[j_{n+1}(k r)\right]^{2}\right\}\left[\frac{\partial P_{n}^{m}(\cos \beta)}{\partial \beta} \cos \gamma\right. \\
\left.+\frac{i m P_{n}^{m}(\cos \beta)}{\sin \beta} \sin \gamma\right] .
\end{gathered}
$$

Equating (A.8) and (A.18) and making use of the definition (2.31)-(2.32), we obtain

$$
p_{-m n}=(-)^{m} i^{-n-1} \frac{2 n+1}{n(n+1)} \underline{G}_{n}^{2 m}(\beta, \alpha) \cdot\left(\cos \gamma \underline{a}_{\beta}+\sin \gamma \underline{a}_{\alpha}\right) .
$$

Finally, using eq (2.30), the expansion coefficient is obtained in the form

$$
p_{m n}=i^{-n-1} \frac{2 n+1}{n(n+1)} \underline{G}_{n}^{2 m *}(\beta, \alpha) \cdot\left(\cos \gamma \underline{a}_{\beta}+\sin \gamma \underline{a}_{\alpha}\right) .
$$

This completes our evaluation for the expansion coefficients of the $\underline{\underline{N}}_{\mathrm{mn}}$ vector spherical-wave functions.

Equations (A.13) and (A.19), in conjunction with the defining equation (A.7), constitute the required vector spherical-wave function series expansion for the incident plane-wave electric field defined by (A.1). This result is in complete agreement with our prior expression eq (2.36) for the series expansion, which was obtained using a dyadic Green's function incident-field representation.

In addition to the previous comparison, an attempt was also made to establish a correspondence with the plane-wave electric-field series-expansion coefficients presented by Bruning and Lo [9]. There, they describe the plane wave's incidence angles with reference to the positive z-axis, while we describe the incidence angles with reference to the negative $\mathrm{z}$-axis; consequently, it is necessary to make the substitutions $\alpha \rightarrow \pi, \beta \rightarrow \pi-\beta_{0}$ in order to transform eqs (A.1) and (A.3) into compatible expressions. However, applying these substitutions to eqs (A.13) and (A.19) did not result in expressions compatible with the expansion coefficients [9, eq (2.14)] given by Bruning and Lo. It would be possible to make our final results agree with theirs only by replacing $\gamma$ with $-\gamma$ in eq (A.13) and simultaneously replacing $\gamma$ with $\pi-\gamma$ in eq (A.19). 
Journal of Research of the National Institute of Standards and Technology-Reports NIST research and development in those disciplines of the physical and engineering sciences in which the Institute is active. These include physics, chemistry, engineering, mathematics, and computer sciences. Papers cover a broad range of subjects, with major emphasis on measurement methodology and the basic technology underlying standardization. Also included from time to time are survey articles on topics closely related to the Institute's technical and scientific programs. Issued six times a year.

\section{Nonperiodicals}

Monographs-Major contributions to the technical literature on various subjects related to the Institute's scientific and technical activities.

Handbooks-Recommended codes of engineering and industrial practice (including safety codes) developed in cooperation with interested industries, professional organizations, and regulatory bodies.

Special Publications-Include proceedings of conferences sponsored by NIST, NIST annual reports, and other special publications appropriate to this grouping such as wall charts, pocket cards, and bibliographies.

Applied Mathematics Series-Mathematical tables, manuals, and studies of special interest to physicists, engineers, chemists, biologists, mathematicians, computer programmers, and others engaged in scientific and technical work.

National Standard Reference Data Series-Provides quantitative data on the physical and chemical properties of materials, compiled from the world's literature and critically evaluated. Developed under a worldwide program coordinated by NIST under the authority of the National Standard Data Act (Public Law 90-396). NOTE: The Journal of Physical and Chemical Reference Data (JPCRD) is published bimonthly for NIST by the American Chemical Society (ACS) and the American Institute of Physics (AIP). Subscriptions, reprints, and supplements are available from ACS, 1155 Sixteenth St., NW, Washington, DC 20056.

Building Science Series-Disseminates technical information developed at the Institute on building materials, components, systems, and whole structures. The series presents research results, test methods, and performance criteria related to the structural and environmental functions and the durability and safety characteristics of building elements and systems.

Technical Notes-Studies or reports which are complete in themselves but restrictive in their treatment of a subject. Analogous to monographs but not so comprehensive in scope or definitive in treatment of the subject area. Often serve as a vehicle for final reports of work performed at NIST under the sponsorship of other government agencies.

Voluntary Product Standards-Developed under procedures published by the Department of Commerce in Part 10, Title 15, of the Code of Federal Regulations. The standards establish nationally recognized requirements for products, and provide all concerned interests with a basis for common understanding of the characteristics of the products. NIST administers this program in support of the efforts of privatesector standardizing organizations.

Consumer Information Series-Practical information, based on NIST research and experience, covering areas of interest to the consumer. Easily understandable language and illustrations provide useful background knowledge for shopping in today's technological marketplace.

Order the above NIST publications from: Superintendent of Documents, Government Printing Office, Washington, DC 20402.

Order the following NIST publications-FIPS and NISTIRs-from the National Technical Information Service, Springfield, VA 22161.

Federal Information Processing Standards Publications (FIPS PUB)-Publications in this series collectively constitute the Federal Information Processing Standards Register. The Register serves as the official source of information in the Federal Government regarding standards issued by NIST pursuant to the Federal Property and Administrative Services Act of 1949 as amended, Public Law 89-306 (79 Stat. 1127), and as implemented by Executive Order 11717 (38 FR 12315, dated May 11, 1973) and Part 6 of Title 15 CFR (Code of Federal Regulations).

NIST Interagency Reports (NISTIR)-A special series of interim or final reports on work performed by NIST for outside sponsors (both government and non-government). In general, initial distribution is handled by the sponsor; public distribution is by the National Technical Information Service, Springfield, VA 22161 , in paper copy or microfiche form. 
National Institute of Standards and Technology 325 Broadway

Boulder, Colorado 80303-3328

Official Business

Penalty for Private Use, $\$ 300$ 